


\title{
Epilepsy Surgery for Focal Cortical Dysplasia: Clinicopathological Correlates and Advances in Neuroimaging
}

\author{
Tim J.Veersema
}


Epilepsy Surgery for Focal Cortical Dysplasia:

Clinicopathological Correlates and Advances in Neuroimaging.

OTim Veersema, 2020

ISBN/EAN: 978-94-6423-011-6

DOI: $10.33540 / 226$

Cover design: Caroline Cracco, Cracco.nl

Layout: Marian Sloot, ProefschriftMaken.nl

print: proefschriftmaken.nl

All rights reserved. No part of this publication may be reproduced in any form or by any means, without permission in writing of the author. The copyright of the articles that have been accepted for publication or that have already been published, has been transferred to the respective journals.

The research in this thesis was financially supported by the Dutch Epilepsy Foundation (subsidy number 12-12).

Financial support for printing and reproduction of this thesis was kindly provided by: ANT Neuro BV, Canon Medical Systems Nederland, ChipSoft, Eisai BV, Goodlife Pharma BV, Guerbet Nederland BV, IQ Medical Ventures BV, Medical Dynamics, Renishaw Benelux BV, Sectra Benelux, UCB Pharma BV and UMC Utrecht Hersencentrum. 


\title{
Epilepsy Surgery for Focal Cortical Dysplasia: Clinicopathological Correlates and Advances in Neuroimaging
}

\author{
Epilepsiechirurgie voor focale corticale dysplasie: \\ clinicopathologische correlaten en ontwikkelingen in \\ neurologische beeldvorming \\ (met een samenvatting in het Nederlands)
}

\section{Proefschrift}

ter verkrijging van de graad van doctor aan de Universiteit Utrecht op gezag van de

rector magnificus, prof.dr. H.R.B.M. Kummeling, ingevolge het besluit van het college voor promoties in het openbaar te verdedigen op

woensdag 25 november 2020 des middags te 12.45 uur

door

\section{Tim Jeroen Veersema}

geboren op 1 juli 1987

te Amsterdam 


\section{Promotoren:}

Prof. dr. K.P.J. Braun

Prof. dr. E.M.A Aronica

\section{Copromotoren:}

Dr. P. van Eijsden

Dr. C.H. Ferrier 


\section{Table of Contents}

Chapter 1 Introduction

Chapter 2 Long-term seizure outcome after epilepsy surgery in patients with mild malformation of cortical development and focal cortical dysplasia

Chapter 3 Cognitive functioning after epilepsy surgery in children with mild malformation of cortical development and focal cortical dysplasia

Chapter 4 Seven tesla MRI Improves detection of focal cortical dysplasia in patients with refractory focal epilepsy

Chapter 57 tesla $T_{2}{ }^{*}$-weighted MRI as a tool to improve detection of focal cortical Dysplasia - a case series

Chapter 6 Changes in vascular density in resected tissue of 97 patients with focal cortical dysplasia or TSC-related cortical tubers

Chapter 7 Correlation between in vivo and ex vivo multimodal high field MRI and histopathological parameters in cortical dysplasias

Chapter 8 Summary and general discussion

Appendices References

Nederlandse samenvatting

Acknowledgements

Dankwoord

Curriculum Vitae 166

List of publications 



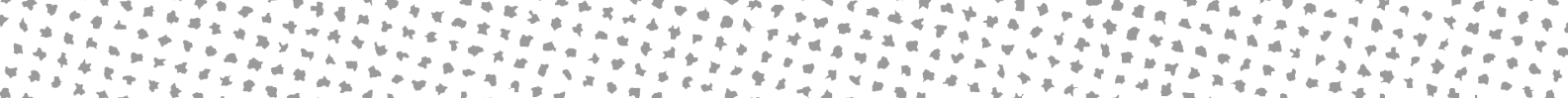

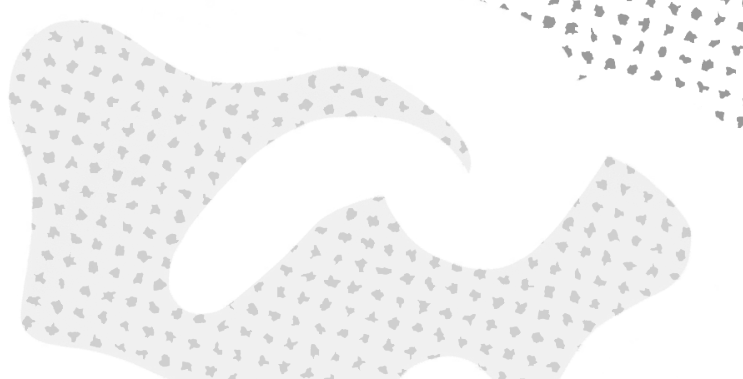

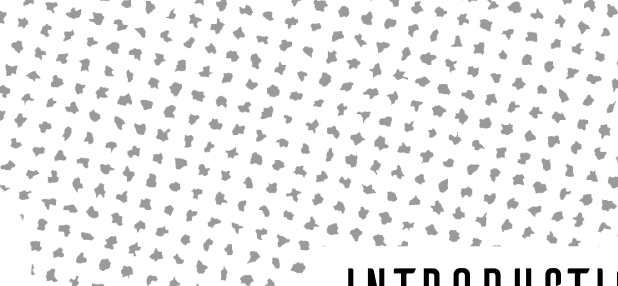

S4

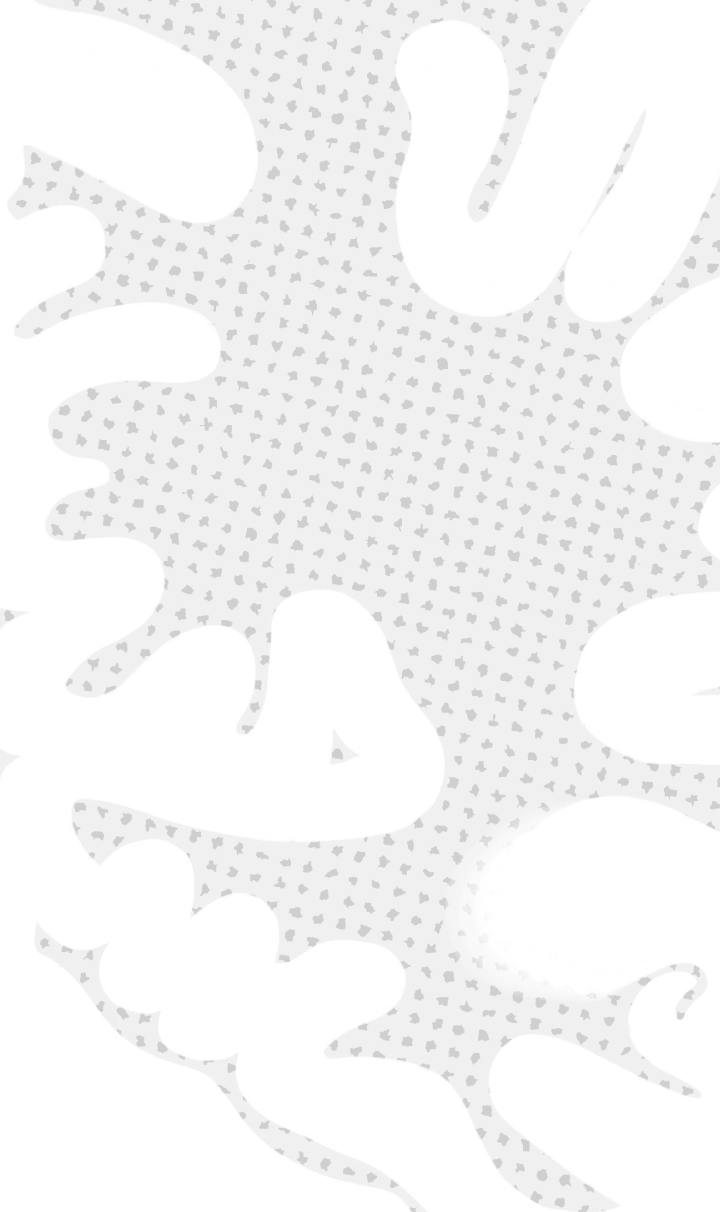
(1)

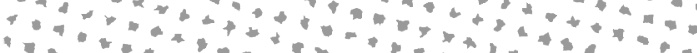

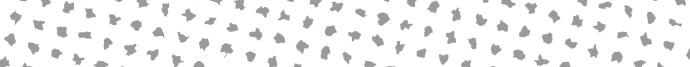

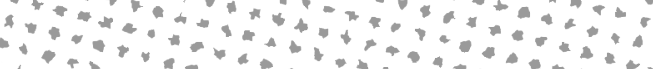

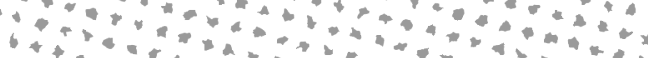

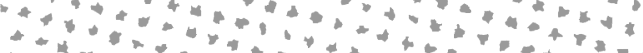
$+1$

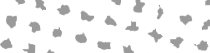
$\cdots+\infty$

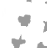
$\div+\div$ $+\ldots$

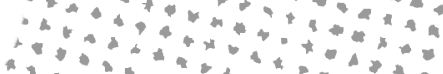

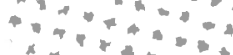
(1)

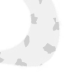
$+\infty$

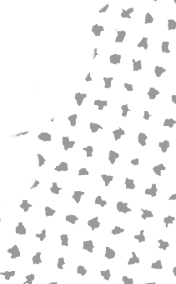
$+\infty$ $+4$ $\rightarrow$

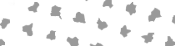
$+\infty$ 
An epileptic seizure is defined as 'a transient occurrence of signs and/or symptoms due to abnormal excessive or synchronous neuronal activity in the brain'. The manifestation is dependent on location in the brain were this abnormal activity originates and the propagation to other brain regions and various other factors. They can manifest as varying combinations of alterations in sensory, motor and autonomic functioning, in cognitive functioning or behaviour. Epilepsy is a brain disorder characterised by a persistent predisposition to generate epileptic seizures and the consequences one experiences ${ }^{1}$. A patient is said to suffer from epilepsy when an epileptic seizure has occurred and the brain demonstrates a enduring tendency to have recurrent seizures, in absence of a temporary or reversible factor that could trigger a seizure (such as seizure directly after a concussion or induced by medication or substances) $)^{2}$. Epilepsy can result from a great variety of underlying brain pathologies that fall under six broad categories: genetic, structural, metabolic, infectious, immune or unknown ${ }^{3}$. In most cases when a patient presents with epilepsy that warrants treatment, initial therapy will be with anti-epileptic medication ${ }^{4,5}$. However, despite of optimal pharmacotherapeutic treatment circa $20-30 \%$ of all patients with epilepsy have persistent seizures ${ }^{6-8}$; these patients are said to have drug resistant or refractory epilepsy ${ }^{9}$. Epilepsy is classified as focal, generalized or combined. This refers to whether the onset of seizures originate from a circumscript brain region or multiple regions in focal epilepsy or conversely that seizures can arise from anywhere in the brain with generalized abnormal electrophysiology ${ }^{3}$. In many patients with focal epilepsy the underlying cause is a developmental abnormality of the brain (falls in structural category), $20 \%$ of all patients who eventually undergo epilepsy surgery $^{10}$. Focal developmental abnormalities can be classified as Focal Cortical Dysplasia (FCD) or mild Malformation of Cortical Development (mMCD) ${ }^{11}$. These are the most common diagnosed pathologies - found in $27 \%$ - among children who undergo epilepsy surgery ${ }^{10}$. The affected area in the cerebral cortex functions abnormally and can generate epileptic activity. Structurally there is disruption of the architecture of the cortex, which normally consists of six layers of neurons, and where the position of the neurons is associated with specific functioning and connections with other neurons. Deep of these six layers is the white matter in which myelinated fibres lie that form the connections with neurons at greater distance within the nervous system. In addition to abnormal layering there can be presence of immature and abnormal neurons.

To harmonize definitions and histopathological assessment the International League Against Epilepsy established a three-tier classification system in $2011^{12}$. Type I is characterized by abnormal layering of the cerebral cortex, either by persistent radial micro-columnar architecture (la) or by a disruption of the horizontal lamination (Ib) or both (IC). Type II is characterized by complete disorganization of the cortex and dysmorphic neurons (Ila), and balloon cells in addition (IIb). FCD type III refers to FCD in combination with other pathologies, such as hippocampal sclerosis (FCD type IIla), glial or glio-neuronal tumours (FCD type IIIb), vascular malformations (FCD type IIIC) or any other principal lesion acquired during early 
life (FCD type IIId). The most subtle architectural abnormalities in the FCD spectrum are classified as $\mathrm{mMCD}$, with intact cortical architecture and absence of aberrant cells, but with excessive number of neurons in the molecular layer (type 1) or white matter (type 2) ${ }^{13}$. This system built on the previous definitions by Palmini ${ }^{14}$ and Taylor ${ }^{15}$. While definitions for ILAE FCD IIb are identical to the previous schemes, more subtle lesions cannot be translated into the new classification system without actual histopathological re-evaluation of tissue samples $^{16}$. See figure 1 for examples of histopathological findings in mMCD/FCD subtypes.

The exact pathogenesis of all FCD subtypes is yet to be completely revealed, but isolated FCD lesions are thought to arise from disruption during early neuronal development, when neurons from the subventricular zone migrate outwards to their position in the cortical ribbon and mature into pyramidal neurons. Depending on the timing of the disruptive events, different aberrant cortical patterns are formed ${ }^{17}$, corresponding with ILAE types. There are indications that increased mammalian target of rapamycin (mTOR) activity plays a role in pathogenesis. mTOR signalling pathways integrate intracellular en extracellular signals and have important regulating functions in cell metabolism, growth, proliferation and survival ${ }^{18}$. Especially for the FCD Ilb subtype, evidence is mounting for these mutations as underlying mechanism with often activation of downstream proteins, and associations with mutations in mTOR-regulatory genes. Similar mTOR dysregulation is found in patients with epilepsy associated with tubers in tuberous sclerosis complex and Hemimegalencephaly ${ }^{17,19}$. Pathogenesis of FCD I and mMCD is more elusive. A limited number of neurons in white matter is considered normal in mature cortex. Excess white matter neurons could represent failure of neuronal migration or de novo generation and it is unclear whether this is de result or consequence of epilepsy ${ }^{17}$.

Patients with FCD typically experience first seizures very early in life, before age of 10 . Seizures are often severe and refractory ${ }^{20}$. Several mechanisms for epileptogenesis of FCD have been suggested. Overexpression of subunits of the glutamate receptors NMDA and AMPA have been found in dysmorphic and dysplastic neurons in FCD, which lead to overexcited states ${ }^{21}$. Other studies found decreased synaptic inhibitory circuits with decreased number of GABAergic neurons and diminished expression of $\mathrm{GABA}_{\mathrm{A}}$ receptor subunits $^{21}$. Also glial dysfunction has been identified in FCD; balloon cells possibly have a hyper-excitability-modifying role through presence of glutamate transporters and the glutamate-metabolizing enzyme glutamine synthase ${ }^{21}$. The close future will see further elucidation of the pathobiology, molecular and electrical characterization of FCDs resulting from the intensive research that is currently carried out.

In patients with epilepsy the burden of disease is not only attributable to epileptic seizures but also to various comorbidities. A specific underlying pathology can be the cause of both epilepsy and the comorbid condition, both also direct causal relations are possible in which 

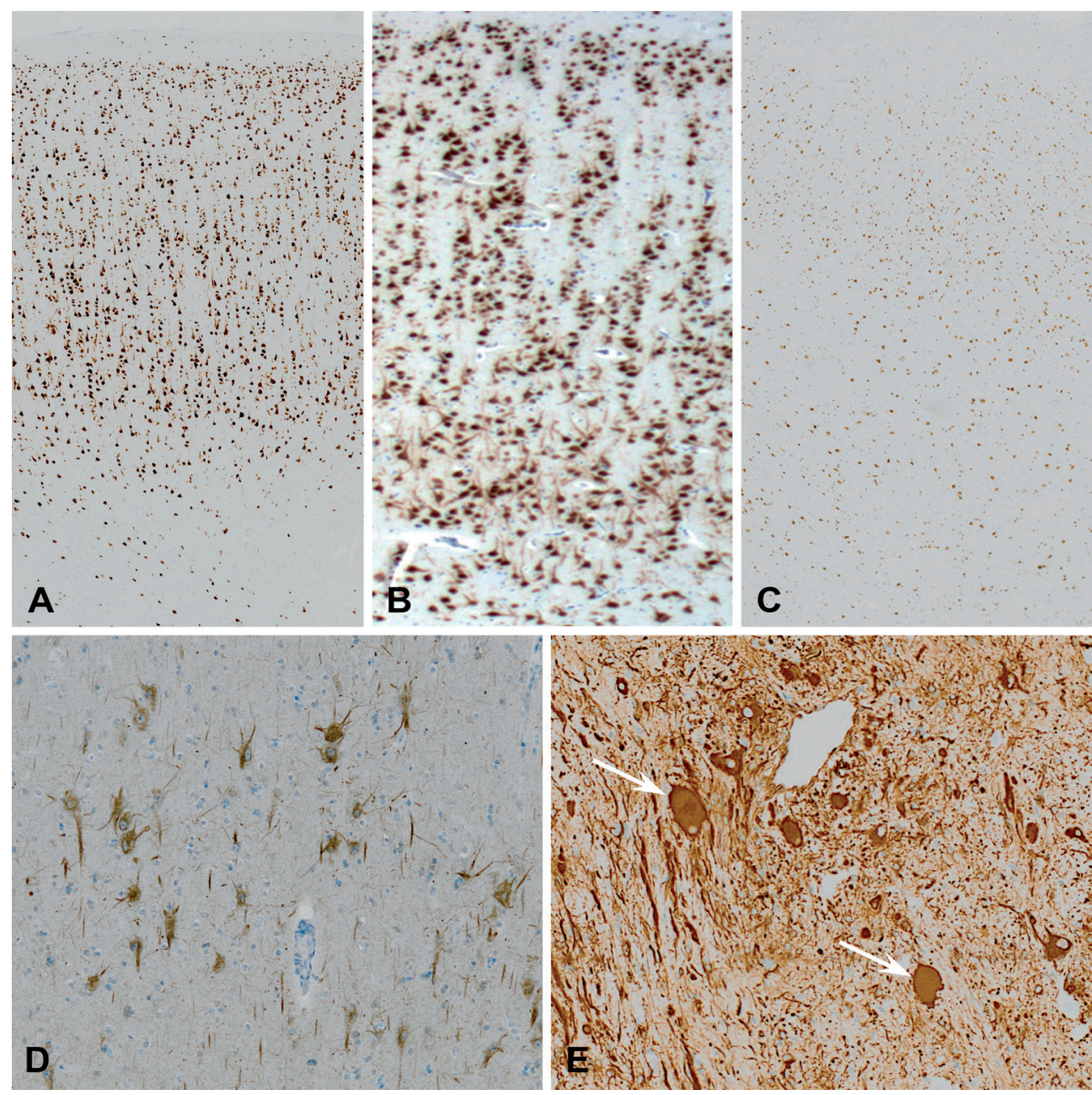

Figure 1. Examples of histological findings in $\mathrm{mMCD}$ and FCD.

A: NeuN/mMCD type 2. Normal cytoarchitecture of cortical layers II-VI, but excess neurons in molecular layer (top of figure) and in white matter (bottom of figure).

B: NeuN/FCD IA. abnormal radial cortical lamination, with typical microcolumnar organisation.

C: NeuN/FCD IIB. In FCD II subtypes there is often complete disruption of cortical layering, and loss of grey/white matter border.

D: SMI32/FCD IIA. Immature, dysmorphic neurons with abnormal orientation and positive staining by SMI32 characterise FCD IIA.

E: Vimentin/FCD IIB. Typical balloon cells, large undifferentiated cells lacking Nissl substance, and positive Vimentin staining.

epileptic activity and/or its treatment are responsible for the comorbidity or vice versa epilepsy is the result of a condition. Not always the mechanisms can be identified, and there can be reciprocity and complex interplay of factors ${ }^{22}$. A wide range of numerous medical comorbidities are known, for instance migraine, cerebrovascular disease and traumatic injury ${ }^{23}$. Considering only epilepsy due to focal developmental abnormalities, the most clinically relevant 
comorbidities are psychiatric and cognitive in nature. Roughly half of the patients with focal epilepsy has at least one psychiatric comorbidity in pre-surgical evaluation. Mood disorders are most common, and also anxiety, personality disorders and psychoses are associated ${ }^{24-26}$. Cognitive disability is common in patients with epilepsy, also in patients with focal aetiology. Around $60 \%$ in paediatric FCD cohorts have cognitive impairment (defined as an intelligence quotient below 70) ${ }^{27,28}$. Moreover, a portion of patients present with epileptic encephalopathy. On top of the disturbances related to the structural anatomy, epileptic activity causes memory consolidation disruption ${ }^{29}$ and in epileptic brain there is a deviation from the optimal network organisation ${ }^{30,31}$. Cognitive disability is not only caused by epileptic activity or the underlying pathology, but also the use of anti-epileptic drugs has negative effects, due to general dampening of neuronal signalling and consequent detrimental effects on cognitive development ${ }^{32}$.

For patients with refractory focal epilepsy, surgery can be a treatment option with prospect of reduction of seizure load or even becoming completely seizure free ${ }^{33}$. On top of this, the possibility of discontinuation of AEDs can be an important argument for surgery, because of inherent and common drug side effects and aforementioned detrimental cognitive effects.

Depending on the location and extent of the lesion in combination with the clinical picture surgery can entail focal resection, anatomical lobectomy, and which may include hippocampectomy. Disconnection of larger epileptogenic zones from normal functioning brain is performed to stop seizure propagation and its damaging effects to areas distant from the ictal onset zone ${ }^{34}$.

Following resective epilepsy surgery in patients with $\mathrm{mMCD} / \mathrm{FCD}$, long term seizure freedom rates of $53 \%-77 \%$ were reported in recent literature ${ }^{35-42}$. This corresponds with Engel 1 scores. A stricter definition is complete and continuous seizure freedom ever since surgery (Engel 1A), which was achieved in $49 \%^{37}$.

Completeness of resection is consistently the most important predictor of seizure freedom. Other factors that have been reported to positively influence chances of seizure freedom are: MRI-visible lesion, temporal lesion, unilobar lesions which facilitate complete resection ${ }^{37,43}$ Shorter epilepsy duration and surgery before adulthood are also associated with seizure freedom ${ }^{37}$. In some studies $\mathrm{mMCD}^{40}$ or $\mathrm{FCD}^{28,44}$ histology has been related to unfavourable outcome, but in others there is no relation with histological subtype ${ }^{35-37,39,41}$. Moreover, there is a paucity of studies that relate outcome to the revised FCD classification. New studies on possible relations between histological subtype and clinical characteristics and outcome after surgery are warranted to validate the new classification scheme. Available surgical studies have limited statistical power due to small sample sizes. The largest long-term outcome study to date included 211 patients with $\mathrm{FCD}^{37}$, most studies were much smaller. 
Blümcke et $\mathrm{al}^{10}$ conducted a multicentre study across 36 centres from 25 European countries, with the primary goal to accurately determine underlying pathologies in epilepsy surgery patients. This is by far the largest epilepsy surgery cohort, but there was limited outcome data, and from a total of 9523 patients, for 5248 (55\%) seizure outcome at 1 year could be used for post hoc analyses to study associations between histology and seizure outcome. No further determinants of seizure outcome were studied.

Most studies reported overall cognitive improvement following epilepsy surgery ${ }^{45-48}$. In general, postoperative cognitive outcome has been shown to correlate with seizure freedom 28,48-51, parental education ${ }^{52}$, preoperative seizure frequency ${ }^{27}$, and with aetiology ${ }^{53,54}$. Importantly, earlier onset ${ }^{27,46,55-62}$ and longer duration ${ }^{27,63}$ of epilepsy predicts more severe cognitive disability. AED policies also affect cognitive outcome $\mathrm{e}^{51,53}$. Significant differences in cognitive outcome between patients with different FCD subtypes have not been reported yet $^{27,42}$.

Good post-surgical outcome in combination with low morbidity and mortality and ongoing advance in surgical techniques subsequently has meant that surgical treatment has an increasingly important role in epilepsy therapy ${ }^{64}$. Currently, surgery is the only treatment option for patients with medically intractable epilepsy to potentially become seizure free. We should therefore strive to be able to offer surgery to as many patients as possible and minimise delay to surgery. Localization of a target is paramount for successful surgery and the diagnostic process for determining surgical candidacy is often extensive, and always includes electroencephalograph (EEG) and structural magnetic resonance imaging (MRI) as first-line investigations. EEG visualises electrophysiological brain activity which are recorded by electrodes placed on the scalp. Epileptic foci express specific electrophysiological patters during (ictal) and between epileptic seizures (interictal). In short, MRI provides structural imaging through magnetic excitation of nuclei and registration of differences in relaxation between tissues and substances ${ }^{65}$. On indication also magnetoencephalography (MEG) or nuclear radiologic imaging such as positron emission tomography (PETCT) and Single Photon Emission Computed Tomography (SPECT) may be performed. If imaging and non-invasive EEG registrations are unable to exactly localize a seizure focus, invasive EEG recordings can be considered; either needles are placed through the skull into the brain tissue (invasive EEG or iEEG with depth electrodes) or the skull is opened to place a mat of electrodes directly on top of the cortical surface (Electrocorticography or ECOG).

MRI plays an increasingly important role in the pre-surgical trajectory. A structural abnormality is a strong and reliable marker for the presence of epileptogenic tissue, even though not always there is perfect co-localisation between a MRI-visible lesion and the epileptogenic zone ${ }^{34}$. A clear and well demarcated lesion obviates more additional 
tests, and provides a target for surgery or invasive registrations. On the other hand, when a lesion is absent, it might be not possible to formulate a hypothesis to base a surgical plan on. Still, successful surgery is possible in MRI-negative patients, but chances of achieving seizure freedom are often found to be smaller ${ }^{33,66-69}$. In recent literaturedepending on subtype-roughly $15 \%$ to $40 \%$ of patients with FCD had a lesion that escaped identification on MRI prior to surgery ${ }^{37,70}$. For mMCD this can be over $50 \%{ }^{42}$. There are number of specific MRI findings associated with mMCD/FCD: most notably blurring of the boundary between grey and white matter and 'transmantle sign' - cone shaped T2 hyperintensity from cortex running towards the ventricle. Other findings are T1 or T2 signal changes of cortex or white matter. Various macro-architectural changes can be seen like thickening of the cortex, abnormal gyral patterns, local cortical atrophy. See figure 2 for examples of MRI findings in FCD. The 'transmantle sign' appears reserved for the ILAE FCD Ilb subtype ${ }^{12}$, whereas FCD Ila, FCD I and MMCD subtypes are harder to discriminate solely based on MRI features ${ }^{71-73}$. There is still uncertainty how specific histological characteristics translate to identifiable MRI features and how the border with normal brain tissue can be identified accurately. Ideally, pre-surgical investigations would already reliably predict the eventual pathohistological diagnosis, as this could influence surgical strategy and improve pre-surgical patient counselling regarding therapeutic outcome. Furthermore, pathological diagnoses and subtyping of $\mathrm{mMCD} / \mathrm{FCD}$ requires adequate positioning and tangential sectioning of the cortex and therefore benefit from accurate pre-surgical imaging diagnoses.

$\mathrm{MRI}$ and image analysis techniques have seen vast advancement, and further improvement is thought to be of great value in improvement of surgical therapies for epilepsy.

In the last decade 7 tesla MRI systems for human use arrived. Compared to the current clinical standard - 1.5 and 3 tesla MRI with dedicated epilepsy scanning protocols - the higher field strength brings an increase in signal to noise ratio which can be utilized to scan with greater contrast, higher resolution or shorter acquisition. With regards to the pre-surgical work up in patients with epilepsy especially improving contrast and resolution have a theoretical benefit for detection of subtle abnormalities. However, higher field strength also brings more artefacts and technical difficulties in producing images of diagnostic quality. Furthermore, the price of purchase of an MRI system is roughly linearly related to magnet field strength. Scientific research is still needed to confirm the clinical benefit of 7T MRI in epilepsy diagnostics.

Since the advent of epilepsy surgery in the end of the $19^{\text {th }}$ century, tremendous changes have taken place in the care for patients with refectory epilepsy. Our understanding of how epileptic seizures are produced, the relation with structural abnormalities and functional anatomy of the brain and how to monitor these has grown exponentially. Surgical techniques have been greatly refined and new methods have been developed. Malformations of Cortical Development make up an increasingly large share of the patient population, and it 

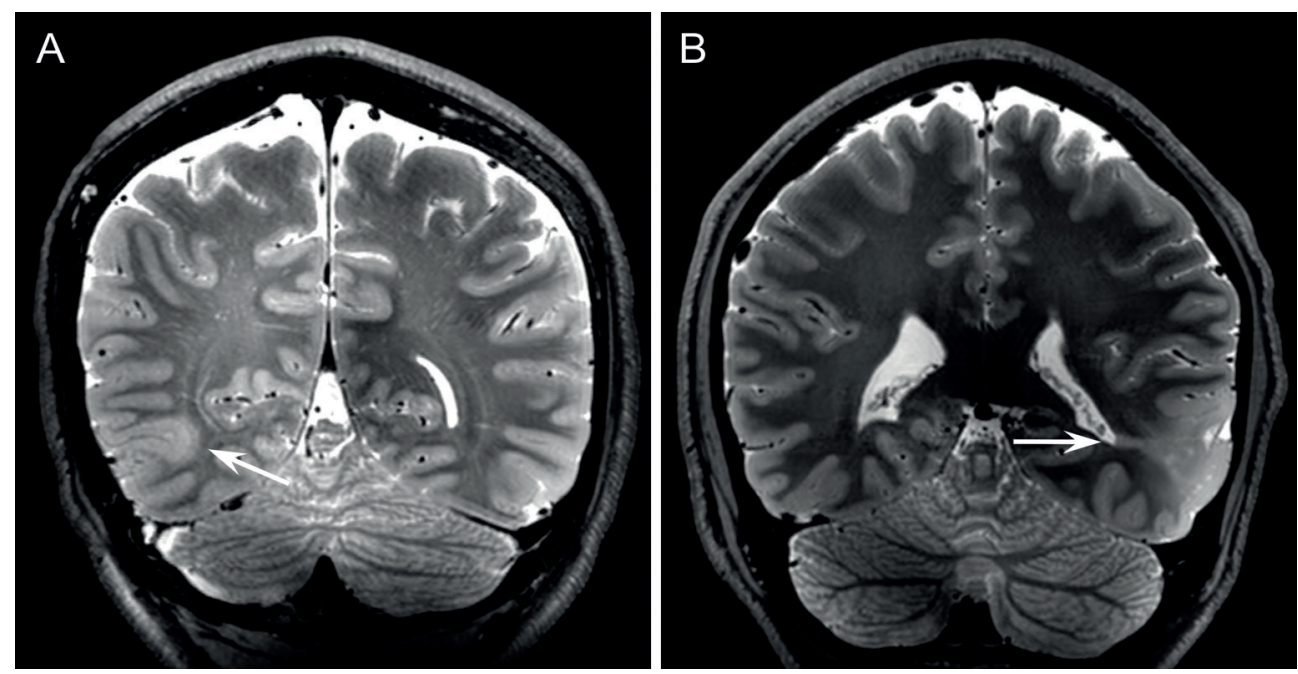

Figure 2. Typical MRI findings in FCD. Coronal reconstructions of 3D $T_{2}$ images at 7T MRI, two patients with FCD Ilb. A: bottom of sulcus dysplasia right parieto-occipital with cortical thickening, blurring of grey-white matter interface and signal changes in cortex and white matter. B: Pronounced FCD lesion in posterior temporal with also typical transmantle sign.

is currently the focus of intensive research to further knowledge and improve quality of care. There remain considerable challenges; in determining the cause of epilepsy, especially in absence of gross structural abnormalities; in identifying the borders between epileptogenic surrounding healthy tissues, when different modalities often provide information that does not perfectly align. Therapeutic decision-making requires a solid a-priori diagnosis and understanding of its repercussion on efficacy and risks of the available treatment options. We aim for this thesis to be a step in this direction. 


\section{Rationale and outline of this thesis}

We describe clinical characteristics and predictors of seizure freedom and cognitive functioning in chapters $\mathbf{2}$ and $\mathbf{3}$ respectively. Although in literature several factors have been brought in association, uncertainty exist concerning determinants in cohorts of only $\mathrm{mMCD} / \mathrm{FCD}$ pathology, and what the influence is of histological subtypes as categorized according to the 2011 ILAE classification.

In chapter 4 we studied the yet to determined clinical value of using 7 tesla MRI in the diagnostic work-up for determining candidacy for epilepsy surgery.

In chapter $\mathbf{5}$ we present the observation of an imaging characteristic of FCD lesions on 7 tesla MRI images, which appears to represent vascular changes co-localizing with FCD lesions and has not previously been described in literature. Expanding on this chapter, and to find a histological substrate, we studied vascular density and relations with blood brain barrier dysfunction, and measures of neuroinflammation in FCD and tubers in TSC in chapter 6.

We conducted a prospective comparative in-vivo 7T MRI - ex-vivo 7T MRI - histology study with as goal to further improve detection, characterisation and delineation of subtle $\mathrm{mMCD} / \mathrm{FCD}$ on MRI. The development of the methodological pipeline and results of image co-registration are described in chapter 7. Full analyses of the MR and histological will be published in the future.

Chapter $\mathbf{8}$ provides a summary of the work collected in this thesis, and a discussion and integration of our research findings. We discuss the implications for patients with $\mathrm{mMCD}$ and FCD, policies concerning epilepsy surgery, and future perspectives.

\section{Aims of this thesis}

- To analyse a surgical cohort of patients with mMCD/FCD and describe clinical characteristics and seizure and cognitive outcome and in relation to the 2011 FCD classification.

- To determine the added value of 7T MRI in the diagnostic work-up for candidates for epilepsy surgery.

- To explore possible new MRI imaging features to improve identification, characterisation and delineation of $\mathrm{mMCD} / \mathrm{FCD}$ lesions. 



\section{LONG-TERM SEIZURE OUTCOME AFTER EPILEPSY SURGERY IN PATIENTS WITH MILD MALFORMATION OF CORTICAL DEVELOPMENT AND FOCAL CORTICAL DYSPLASIA}

Tim J. Veersema, Banu Swampillai, Cyrille H. Ferrier, Pieter van Eijsden Peter H. Gosselaar, Peter C. van Rijen, Wim G.M. Spliet, Angelika Mühlebner Eleonora Aronica, Kees P.J. Braun 
Focal Cortical Dysplasia (FCD) and mild malformation of cortical development (mMCD) are frequent histopathological diagnoses in patients who undergo surgery for refractory epilepsy. Literature concerning surgical outcome in patients with $\mathrm{mMCD}$, as well as its contrast with FCD, has been scarce.

We studied 88 patients with a histopathological diagnosis of isolated FCD $(n=57)$ or $m M C D(n=31)$, revised according to the latest ILAE guidelines, who underwent resective or disconnective surgery

Our findings suggest differences between $\mathrm{mMCD}$ and FCD in clinical presentation and surgical outcome after surgery. Patients with mMCD developed seizures later in life, and their lesions had a predilection for location in the temporal lobe, and remained undetected by MRI more frequently. A diagnosis of $\mathrm{mMCD}$ has a less favorable surgical outcome. Still, $32 \%$ of these patients reached continuous seizure freedom (Engel $1 \mathrm{~A}$ ) at a latest median follow-up duration of 8 years, compared to $59 \%$ in FCD. A histopathological diagnosis of $\mathrm{mMCD}$, extra-temporal surgery and indication of an incomplete resection each were independent predictors of poor outcome. 


\section{Introduction}

Focal cortical dysplasia (FCD) and mild malformation of cortical development (mMCD) are frequent histopathological diagnoses in patients who undergo epilepsy surgery ${ }^{10}$. The 2011 ILAE classification system differentiates FCD type I and II as isolated lesions from type III, which is associated with adjacent other pathology ${ }^{12}$. The most subtle architectural abnormalities in the FCD spectrum are classified as MMCD, with intact cortical architecture and absence of aberrant cells, but with excessive number of neurons in the molecular layer (type 1) or white matter (type 2$)^{14}$.

Several factors have been reported to predict postoperative seizure freedom in mMCD/ FCD, of which complete resection is most consistently identified ${ }^{37,43}$. In the few surgical outcome studies that also included mMCD worse outcome was suggested for $\mathrm{MMCD}$ and FCD type I, compared to type II FCD ${ }^{39,40}$.

Although mMCD is relatively under-exposed in recent literature, it is a common diagnosis with $2.9 \%$ of all epilepsy surgery cases, compared to $2.8 \%$ and $9 \%$ for FCD I and II respectively ${ }^{10}$. We aimed to elucidate differences in clinical characteristics and seizure outcomes between mMCD and FCD subtypes, and identify determinants of postoperative seizure freedom.

\section{Methods}

The pathological diagnosis of all 634 patients who underwent epilepsy surgery between 2000 and 2012 in our center and had a histopathological report compatible with FCD or mMCD (e.g., malformation of cortical development [MCD], microdysgenesis, dysplasia) were reviewed according to the 2011 ILAE classification ${ }^{13}$. To avoid effects of associated pathology on outcome, patients with complex MCD, neurocutaneous syndromes, and FCD III subtypes were not included.

A lesionectomy - preferably en-bloc - was performed for distinct lesions. Intra-operative electrocorticography (ECOG) was used on indication to tailor resection. Disconnective surgery (functional hemispherectomy or frontal or posterior disconnection) was indicated in more widespread or multilobar seizure onset zones. Patients were only included when a tissue sample of the disconnected area was sent for pathological investigation. The revised diagnosis was based on the following immunohistochemical stainings: hematoxylin \& eosin ( $\mathrm{H} \& \mathrm{E})$, neuronal nuclei, antibody neurofilament $\mathrm{H}$ non-phosphorylated, Vimentin, glial fibrillary acidic protein, and microtubule- associated protein 2 . All samples were revised (AM and TV) and - if necessary - reclassified according to the 2011 ILAE classification system. In case of non-consensus the senior pathologist (EA) was consulted. 
Age at epilepsy onset and surgery, seizure duration, generalized tonic-clonic seizures (GTCS) ever to have occurred, seizure frequency (daily or less frequent), location of surgery (frontal, temporal, parietal, occipital, multilobar), magnetic resonance imaging (MRI) lesion identified (visual assessment only), and surgery type (lesionectomy, lobectomy, (sub)lobar disconnection, functional hemispherectomy) were collected from patients' files. Indication for incomplete resection was based on intra-operative assessment by the surgeon, ECOG, or on post-operative MRI (if performed).

Information on post-surgical seizure and medication status was collected by telephone interview, 2-14 years following surgery.

Seizure outcome was classified as seizure freedom (completely seizure-free ever since surgery [Engel class 1a]) or not (all other Engel classes) at last follow-up. Acute postoperative seizures during the first month were not taken into consideration. In addition, seizure- and medication-freedom at last follow-up was noted ("Engel 1a AED-"). In case of a reoperation within 2 years, seizure outcomes were measured after the second surgery. The tissue sample with most obvious pathological features was considered for the definitive histopathological diagnosis.

\section{Analysis}

Ages at onset and surgery, and duration of epilepsy and follow-up failed tests for normality, therefore Mann-Whitney $U$ was used to study differences between pathologies and test influence on outcome. Fisher exact tests were used to examine differences in MRI visibility, age at surgery (dichotomized at $<$ or $\geq 18$ years), presence of GTCS, site of lesion, indication for incomplete resection, seizure freedom and cure, between FCD subtypes. Logistic regression was used for multivariate analysis of seizure outcome. To reduce multicollinearity several determinants were not analyzed in multivariate logistic regression and location was dichotomized into extra-temporal (including multilobar) and temporal surgery. By only including the following variables, variance inflation factors are kept below 2.5 and condition index below 15: sex, MRI negative, only focal versus also GTCS, daily seizures, duration of epilepsy, lateralization, extra-temporal surgery, indication for incomplete resection, and histopathological diagnosis mMCD versus FCD. 


\section{Results}

Eighty-eight consecutive patients were included, of whom 11 (13\%) underwent two intracranial surgical procedures, seven (8\%) within 2 years after first surgery. Revised histology was mMCD type $1(n=6)$, mMCD type $2(n=25)$, FCD la $(n=3)$, FCD lb $(n=4)$, FCD Ic $(n=1)$, FCD Ila $(n=20)$, FCD Ilb $(n=29)$.

Clinical characteristics of pathology categories are listed in Table 1. Seizure onset was significantly later in mMCD (median 10 years) compared to FCD (median 3 years). Age at surgery was older in mMCD (median 21.8 years) than in FCD (median 13.7 years).

FCD was more often localized in the frontal lobes $(60 \%)$ compared to $\mathrm{mMCD}(23 \%)$, although FCD I subtypes (25\%) had a similar distribution to mMCD. mMCD was more frequently located in the temporal lobe (55\%) compared to FCD (18\%).

A significantly larger portion of $\mathrm{mMCD}(45 \%)$ lesions was not detected on MRI compared to FCD (16\%). See figure 1 for an example of MRI lesions in MMCD.

\section{Seizure outcome}

Outcome data was missing for one patient. For the remaining 87, follow-up ranged 2-14 years (median 7 years). Follow-up duration was similar between histological subtypes. Fortythree patients (49\%) reached continuous complete seizure freedom at last follow-up, and 31 (36\%) were also free of anti-epileptic medication (Supplementary table 1).

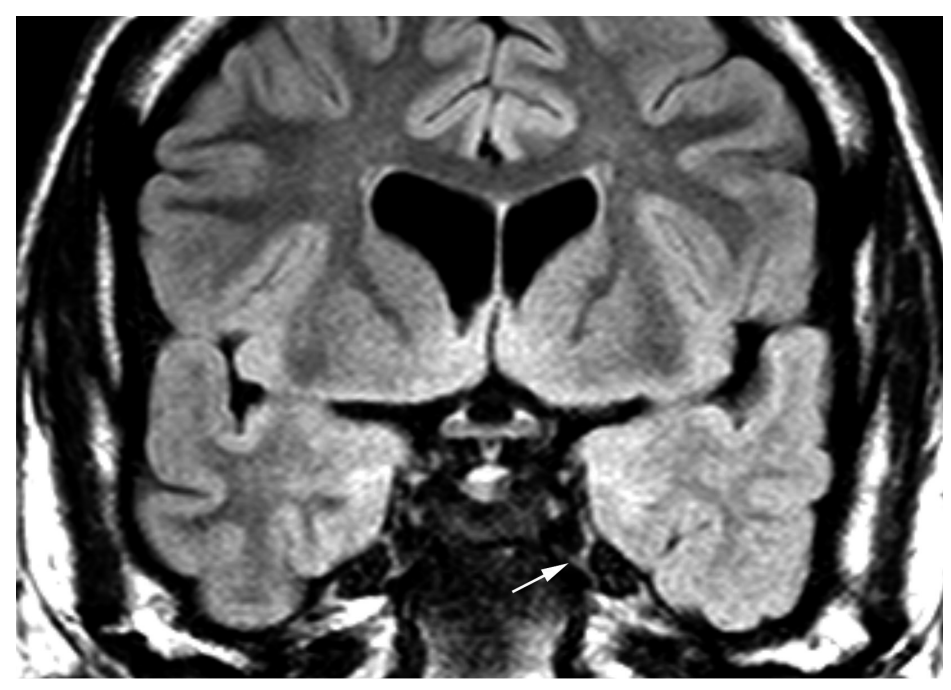

Figure 1. Patient diagnosed with $\mathrm{mMCD}$ type 2 . Onset at 16 years, with complex partial seizures and secondarily generalized tonic-clonic seizures. Coronal 3T MRI FLAIR images revealed blurring of gray-white matter interface in left temporal lobe. No indication for hippocampal sclerosis. Patient underwent anterior temporal lobe resection at age 19 years. Engel score $1 \mathrm{C}$ at ten years follow-up. Recurrence at seven years, and again achieving seizure freedom with medication since. 
Table 1. Clinical characteristics and seizure outcome in relationship to histological subtype.

\begin{tabular}{|c|c|c|c|c|c|c|c|}
\hline & $\begin{array}{l}\text { Total } \\
\mathrm{n}=\mathbf{8 8}\end{array}$ & $\begin{array}{l}\mathrm{mMCD} \\
\mathrm{n}=31 \\
(35 \%)\end{array}$ & $\begin{array}{l}\text { FCD } \\
\text { total } 57 \\
(65 \%)\end{array}$ & $\begin{array}{l}\text { FCD I } \\
8(9 \%)\end{array}$ & $\begin{array}{l}\text { FCD Ila } \\
20(23 \%)\end{array}$ & $\begin{array}{l}\text { FCD IIb } \\
29(33 \%)\end{array}$ & $\begin{array}{l}\text { Statistics } \\
\text { mMCD } \\
\text { vs FCD }\end{array}$ \\
\hline \multicolumn{8}{|l|}{ Baseline characteristics } \\
\hline Sex, female & $43(49 \%)$ & $17(55 \%)$ & $26(46 \%)$ & $5(63 \%)$ & $6(30 \%)$ & $15(52 \%)$ & $p=0.504$ \\
\hline $\begin{array}{l}\text { Age at epilepsy onset, } \\
\text { median and range } \\
\text { (years) }\end{array}$ & $6(0-38)$ & $10(0-38)$ & $3(0-26)$ & $4.5(0-17$ & $3.5(0-22)$ & $3(0-26)$ & $p=0.001$ \\
\hline $\begin{array}{l}\text { Age at surgery, } \\
\text { median and range } \\
\text { (years) }\end{array}$ & $\begin{array}{l}15.8 \\
(0.23-48.1)\end{array}$ & $\begin{array}{l}21.8(1.0- \\
48.1)\end{array}$ & $\begin{array}{l}13.7(0.2- \\
45.0)\end{array}$ & $\begin{array}{l}9.7(0.55- \\
40.3)\end{array}$ & $\begin{array}{l}13.0 \\
(0.23- \\
44.9)\end{array}$ & $\begin{array}{l}14.5 \\
(0.74- \\
45.0)\end{array}$ & $p=0.022$ \\
\hline $\begin{array}{l}\text { Duration of epilepsy, } \\
\text { median and range } \\
\text { (years) }\end{array}$ & $8(0-37)$ & $9(1-33)$ & $8(0-37)$ & $4.5(1-23)$ & $5(0-37)$ & $10(1-36)$ & $p=0.500$ \\
\hline $\begin{array}{l}\text { Generalized tonic- } \\
\text { clonic seizures }\end{array}$ & $61 / 88(69 \%)$ & $19(61 \%)$ & 42 (74\%) & $6(75 \%)$ & 15 (75\%) & $21(72 \%)$ & $p=0.238$ \\
\hline Daily seizures & $52 / 88(59 \%)$ & $13(42 \%)$ & $39(68 \%)$ & $6(75 \%)$ & $10(50 \%)$ & $23(79 \%)$ & $p=0.023$ \\
\hline Site of surgery & & & & & & & $p<0.001$ \\
\hline Frontal surgery & $41(47 \%)$ & $7(23 \%)$ & $34(60 \%)$ & $2(25 \%)$ & $12(60 \%)$ & $20(69 \%)$ & $p=0.002$ \\
\hline Temporal surgery & $27(31 \%)$ & $17(55 \%)$ & $10(18 \%)$ & $3(38 \%)$ & $4(20 \%)$ & $3(10 \%)$ & $p=0.001$ \\
\hline Parietal surgery & $6(7 \%)$ & 0 & $6(11 \%)$ & 0 & $2(10 \%)$ & $4(14 \%)$ & $p=0.086$ \\
\hline Occipital surgery & $2(2 \%)$ & $1(3 \%)$ & $1(2 \%)$ & 0 & 0 & $1(3 \%)$ & $p=1.000$ \\
\hline multilobar surgery & $12(14 \%)$ & $6(19 \%)$ & $6(11 \%)$ & $3(38 \%)$ & $2(10 \%)$ & $1(3.4 \%)$ & $p=0.331$ \\
\hline $\begin{array}{l}\text { Lateralization, left } \\
\text { sided }\end{array}$ & $39 / 88(44 \%)$ & $15(48 \%)$ & $24(42 \%)$ & $3(38 \%)$ & $9(45 \%)$ & $12(41 \%)$ & $p=0.655$ \\
\hline MRI-negative lesion & $23 / 88(26 \%)$ & $14(45 \%)$ & $9(16 \%)$ & $4(50 \%)$ & $4(20 \%)$ & $1(3.4 \%)$ & $p=0.005$ \\
\hline $\begin{array}{l}\text { Resective surgery (vs } \\
\text { disconnective surgery }\end{array}$ & $76 / 88(86 \%)$ & $26(84 \%)$ & $50(88 \%)$ & $4(50 \%)$ & $18(90 \%)$ & $28(97 \%)$ & $p=0.747$ \\
\hline $\begin{array}{l}\text { long-term invasive } \\
\text { monitoring }\end{array}$ & $41 / 88(47 \%)$ & $14(45 \%)$ & 27 (47\%) & $1(13 \%)$ & $9(45 \%)$ & $17(59 \%)$ & $p=1.000$ \\
\hline $\begin{array}{l}\text { Indication for } \\
\text { incomplete resection } \\
\left(1^{\text {st }} \text { surgery }\right)\end{array}$ & 16/76 (18\%) & $4(13 \%)$ & $12(21 \%)$ & $2(25 \%)$ & $2(10 \%)$ & $8(28 \%)$ & $p=0.400$ \\
\hline $\begin{array}{l}\text { Re-operations (intra- } \\
\text { cranial surgery) }\end{array}$ & $\begin{array}{l}11 / 88 \\
(12.5 \%) \\
\end{array}$ & $3(25 \%)$ & $8(14 \%)$ & $2(25 \%)$ & $1(5 \%)$ & $5(17 \%)$ & $p=0.740$ \\
\hline \multicolumn{8}{|l|}{ Outcome } \\
\hline Engel $1 \mathrm{~A}$ & 43/87 (49\%) & $10(32 \%)$ & $33(59 \%)$ & $4(50 \%)$ & $10(53 \%)$ & $19(66 \%)$ & $p=0.025$ \\
\hline Engel 1A AED- & $31 / 87$ (36\%) & $7(23 \%)$ & $24(43 \%)$ & $3(38 \%)$ & $7(36.8 \%)$ & $14(48 \%)$ & $p=0.066$ \\
\hline $\begin{array}{l}\text { Follow-up duration, } \\
\text { median and range } \\
\text { (years) }\end{array}$ & $7(2-14)$ & $6(2-12)$ & $7(0-14)$ & $7.5(3-13)$ & $7(3-14)$ & $5(2-14)$ & $p=0.682$ \\
\hline
\end{tabular}

Clinical features and postoperative outcome in relation to FCD subtypes. Fisher's exact for categorical variables and Mann-Whitney $U$ test for continuous non-parametric variables. 
Seizure freedom was significantly more common after surgery for FCD (59\%) than for mMCD (32\%). Patients with mMCD tended to be less likely free of seizures and medication (23\%) compared to FCD (43\%). Results of logistic regression analysis are displayed in table 2. Indication of incomplete surgery and mMCD diagnosis (versus FCD) both univariately negatively predicted continuous seizure freedom at last follow-up. In multivariate logistic regression analyses, both $\mathrm{mMCD}$ and incomplete resection remained significant predictors, as did extratemporal resection. In multivariate analysis only the presence of daily seizures predicted seizure- and medication freedom at last follow up. Older age at surgery and presence of daily seizures were negative predictors in univariate analysis.

Table 2. Determinants of favorable seizure outcome.

Univariate Odds ratio of determinant for favorable outcome

\begin{tabular}{lllll}
\hline & Engel 1A & $\mathbf{9 5 \%} \mathbf{C l}$ & Engel 1A AED- & $\mathbf{9 5 \%} \mathbf{C l}$ \\
\hline Age at surgery (years) & NS & NS & $\mathbf{0 . 9 6 *}$ & $\mathbf{0 . 9 2 - 1 . 0 0}$ \\
\hline Daily seizures & NS & NS & $\mathbf{3 . 4 3 *}$ & $\mathbf{1 . 2 7 - 9 . 2 4}$ \\
\hline $\begin{array}{l}\text { Indication for incomplete } \\
\text { resection, (only resective } \\
\text { surgery }\end{array}$ & $\mathbf{0 . 1 8 *}$ & $\mathbf{0 . 0 4 - 0 . 9 2}$ & NS & NS \\
\hline \begin{tabular}{l} 
mMCD (vs FCD) \\
\hline
\end{tabular} & $\mathbf{0 . 3 3 ^ { * }}$ & $\mathbf{0 . 1 3 - 0 . 8 4}$ & $0.39^{t}$ & \\
\hline
\end{tabular}

Multivariate Odds ratio of determinant for favorable outcome

\begin{tabular}{lllll}
\hline & Engel 1A & $\mathbf{9 5 \%} \mathbf{C l}$ & Engel 1A AED- & $\mathbf{9 5 \%} \mathbf{C l}$ \\
\hline Daily seizures & NS & NS & $\mathbf{4 . 0 6 *}$ & $\mathbf{1 . 1 3 - 1 4 . 6 0}$ \\
\hline Extra-temporal surgery & $\mathbf{0 . 2 0 *}$ & $0.05-0.88$ & NS & NS \\
\hline $\begin{array}{l}\text { Indication for Incomplete } \\
\text { resection }\end{array}$ & $\mathbf{0 . 2 0 *}$ & $0.04-0.99$ & NS & NS \\
\hline mMCD (vs FCD) & $\mathbf{0 . 1 2 * *}$ & $\mathbf{0 . 0 3 - 0 . 4 4}$ & $0.3^{5} \mathrm{t}$ & $0.11-1.14$ \\
\hline
\end{tabular}

${ }^{*}: p \leq 0.05,{ }^{* *}: p \leq 0.05,{ }^{t}:$ trend, $p \leq 0.1, N S:$ not significant, $p>0.1$

Univariate logistic regression, odds ratios and $95 \%$ confidence intervals. Determinants with p>0.1 omitted from table: female sex, Age at epilepsy onset (years), Duration of epilepsy (years), generalized tonic-clonic seizures, right-sided, extra-temporal, frontal, temporal, parietal, occipital, multilobar surgery, MRI negative lesion, disconnective surgery, follow-up duration.

multivariate logistic regression: entry of determinants for seizure outcome with constraints by multicollinearity. Determinants without significant relation with any outcome measure are omitted from table: sex, epilepsy duration, MRI-negative lesion, and bilateral seizures.

\section{Discussion}

For 31 of 88 (35\%) of all patients with isolated malformations of cortical development - those with complex and neurocutaneous disorders excluded - mMCD was a common diagnosis, while FCD I was relatively rare with only $9 \%$. FCD II was found in over half of the patients (FCD IIb 33\%, IIA 23\%). In a recent multicenter study which included 1612 patients with 
mMCD and FCD, FCD II was equally frequent (53\%) but mMCD was not more common than FCD I (both $17 \%)^{10}$. However, $13 \%$ of patients with MCD had lesions reported as "FCD not otherwise specified". Moreover, difference in FCD subtyping was thought to have arisen due the lack of international classification system before the ILAE consensus of 2011. Recent consecutive cohort studies including adult patients, in which tissue was (re)classified according to ILAE histopathological classification, did not include mMCD diagnoses, and the ratio between FCD I and FCD II varied from $26 \% / 74 \%$ to $44 \% / 56 \%{ }^{35-37}$. In our cohort the proportion of FCD I was relatively low. There are several explanations for these differences in presented histology. Observer variability possibly remains despite efforts to harmonize histopathological classification, and with current definitions especially the milder pathologies can be debatable. No formal inclusion of mMCD in the ILAE classification system might compel to classify very subtle dysplastic lesions as FCD I, when mMCD would be more suitable. Discrepancy in utilization of immunohistochemical methods may also play a role in variability in subclassification. Although ILAE Type II FCD is an established entity, there is still debate concerning the definitive identifying histological and contrasting clinical features of $m M C D$ versus FCD I ${ }^{74}$.

Our findings suggest that clinical characteristics help differentiating patients with mMCD from those with other FCD subtypes. They had onset of epilepsy later in life and consequently were operated at older age, the majority (61\%) even at adult age, whereas only $32 \%$ of patients with FCD were operated during adulthood. Epilepsy duration was not significantly different between histological subtypes.

Younger age at onset and surgery in patients with FCD II compared to FCD I has been reported previously ${ }^{35}$. Patients with $\mathrm{mMCD}$ were less likely to have highly frequent (daily) seizures. As reported in previous studies $35,75,76$ we observed a predilection of FCD II types for the frontal lobe, whereas mMCD and FCD I were most often localized in the temporal lobes. In case of multilobar lesions, mMCD and FCD I were most often diagnosed.

In our series, epilepsy surgery resulted in a continuing seizure freedom rate of $49 \%$ at a median follow-up of 7 years; $36 \%$ had also discontinued anti-epileptic medication. Patients with $\mathrm{mMCD}$ were less likely to reach seizure freedom. Patients with FCD Ilb diagnoses had distinctively higher chance of favorable outcome. A number of studies did not show significant differences in seizure outcome between different histological subtypes ${ }^{35-37,41}$. It must be noted that no, or very few, mMCD cases were included in these studies. Pathology was neither a predictor of outcome in a study that did include 29 mMCD cases ${ }^{39}$. In a study by Kim et al. more patients with FCD $(85 / 145,59 \%)$ were seizure free at last follow up compared to $\mathrm{mMCD}(9 / 21,43 \%)^{40}$. Others studies, not including $\mathrm{mMCD}$, showed less favorable outcome in patients with FCD I histology ${ }^{28,44}$. The inferior outcome after surgery in $\mathrm{mMCD}$ is likely due to difficulties in determining location and margins on imaging and during surgery, complicating accurate identification and complete removal of the epileptogenic zone. Even though we 
did not see indication for incomplete resection more often in patients with $\mathrm{mMCD}$, residual lesions are most likely the best explanation for the difference in seizure outcome, albeit that these might not be suspected based on intra-operative observations by the surgeon, ECOG or post-surgical imaging. Complete resection of the lesion is the most reported predictor of favorable outcome, along with factors that facilitate this: temporal focus, severe pathological features, and a MRI-defined lesion ${ }^{43}$. Accordingly, in our multivariate analyses, $\mathrm{mMCD}$, extratemporal surgery and indication for incomplete resection were predictors of unfavorable seizure outcome. We could not reproduce a relation between absence of an MRI lesion and surgical outcome, neither when analyzing histological subgroups separately. Patients who suffered from highly frequent, daily seizures, were more likely to discontinue medication after complete seizure freedom. An explanation might be that in these patients the positive effect on daily life after successful surgery is more outspoken and is an encouragement for earlier discontinuation of medication.

\section{Conclusion}

Our results suggest $\mathrm{mMCD}$ has a differentiating clinical presentation, which may be relevant in predicting seizure outcome after epilepsy surgery. Patients with $\mathrm{mMCD}$ develop seizures later in life, compared to FCD ILAE type I and II. mMCD has a predilection for temporal lobes and remains undetected by MRI more frequently. A diagnosis of $\mathrm{mMCD}$ has a less favorable surgical outcome. Nonetheless, one-third of these patients are expected to reach complete and ongoing seizure freedom, thus making the consideration of surgical treatment worthwhile.

Supplementary table 1. Engel scores at 2-year intervals.

\begin{tabular}{lllllllll}
\hline \multicolumn{7}{l}{ years of follow up } & \multicolumn{1}{l}{} \\
\cline { 2 - 8 } & $\mathbf{2}$ & $\mathbf{4}$ & $\mathbf{6}$ & $\mathbf{8}$ & $\mathbf{1 0}$ & $\mathbf{1 2}$ & $\mathbf{1 4}$ & latest \\
\hline Engel 1a & $52(60 \%)$ & $41(55 \%)$ & $30(60 \%)$ & $19(56 \%)$ & $8(47 \%)$ & $5(50 \%)$ & $1(50 \%)$ & $43(49 \%)$ \\
\hline Engel 1 & $56(64 \%)$ & $46(62 \%)$ & $33(66 \%)$ & $21(62 \%)$ & $12(71 \%)$ & $6(60 \%)$ & $1(50 \%)$ & $54(62 \%)$ \\
\hline Engel 2 & $5(6 \%)$ & $4(5 \%)$ & $3(6 \%)$ & $4(12 \%)$ & $1(6 \%)$ & $2(20 \%)$ & 0 & $6(7 \%)$ \\
\hline Engel 3 & $17(20 \%)$ & $14(19 \%)$ & $9(18 \%)$ & $6(18 \%)$ & $4(24 \%)$ & $2(20 \%)$ & 0 & $15(17 \%)$ \\
\hline Engel 4 & $9(10 \%)$ & $10(14 \%)$ & $5(10 \%)$ & $3(9 \%)$ & 0 & 0 & $1(50 \%)$ & $12(14 \%)$ \\
\hline Total & 87 & 74 & 50 & 34 & 17 & 10 & 2 & 87 \\
\hline
\end{tabular}

Engel scores at 2-year intervals after surgery 



\section{COGNITIVE FUNCTIONING AFTER EPILEPSY SURGEAY IN CHILDREN WITH MILD MALFORMATION OF CORTICAL DEVELOPMENT AND FOCAL CORTICAL DYSPLASIA}

Tim J. Veersema, Monique M.J. van Schooneveld, Cyrille H. Ferrier Pieter van Eijsden, Peter H. Gosselaar, Peter C. van Rijen, Wim G.M. Spliet Angelika Mühlebner, Eleonora Aronica, Kees P.J. Braun 
Mild malformation of cortical development (mMCD) and focal cortical dysplasia (FCD) subtypes combined are by far the most common histological diagnoses in children who undergo surgery as treatment for refractory epilepsy. In patients with refractory epilepsy a substantial burden of disease is due to cognitive impairment. We studied IQ or developmental quotient (DQ) values, and their change after epilepsy surgery in a consecutive series of 42 children (median age at surgery 4.5 , range 0-17.0 years) with refractory epilepsy due to $\mathrm{mMCD} / \mathrm{FCD}$. Cognitive impairment, defined as IQ/DQ below 70 , was present in $51 \%$ prior to surgery. Cognitive impairment was associated with earlier onset of epilepsy, longer epilepsy duration and FCD type I histology. Clinically relevant improvement of $\geq 10 \mathrm{IQ} / \mathrm{DQ}$ points was found in $24 \%$ of children and was related to the presence of presurgical epileptic encephalopathy. At time of post-surgical cognitive testing 59\% children were completely seizure free (Engel 1A). We found no association between cognitive outcome and seizure or medication status at two years follow-up. Epilepsy surgery in children with mMCD or FCD not only is likely to result in complete and continuous seizure freedom, but also improves cognitive function in many. 


\section{Introduction}

Focal cortical dysplasia (FCD) subtypes, including minimal malformation of cortical development (mMCD), are by far the most common histological diagnoses in children who undergo epilepsy surgery ${ }^{10}$. In FCD focal cortical abnormalities are characterized by aberrant organization of cortical layers (type I), in which also abnormal neurons can occur (type II) $)^{13}$. The most subtle focal architectural abnormalities in the malformation of cortical development spectrum are classified as $\mathrm{MMCD}$, with intact cortical architecture and absence of aberrant cells, but with excessive number of neurons in the molecular layer (type 1) or white matter (type 2) ${ }^{14}$.

An important comorbidity in children and adults with refractory epilepsy is developmental delay. The underlying pathology, epileptic activity, and use of anti-epileptic medication all contribute to cognitive disturbances ${ }^{32}$. In children with refractory epilepsy due to FCD, 53-68\% have cognitive impairment $(\mathrm{IQ}<70)^{27,28}$. In children with so-called epileptic encephalopathy, in which the epileptic activity further decreases the child's cognitive developmental abilities, early treatment is warranted to improve cognitive outcome ${ }^{77}$.

Most studies reported overall cognitive improvement following epilepsy surgery ${ }^{45-48}$. In children with severe epilepsy who undergo surgery at young age and who often had preoperative developmental arrest or regression, clinically relevant increases in IQ scores of e.g. 7-10 or more points are often not achieved, although the child's cognitive age clearly starts to increase after surgery ${ }^{77,78}$.

In general, postoperative cognitive outcome has been shown to correlate with seizure freedom ${ }^{28,48-51}$, parental education ${ }^{52}$, anti-epileptic drug (AED) policies ${ }^{51,53}$, preoperative seizure frequency ${ }^{27}$, and with aetiology ${ }^{53,54}$. Importantly, earlier onset ${ }^{27,46,55-62}$ and longer duration ${ }^{27,63}$ of epilepsy predicts more severe cognitive impairment. Significant differences in cognitive outcome between patients with different FCD subtypes have not yet been reported ${ }^{27,42}$.

The aim of this study is to assess pre- and postoperative cognitive functioning in children with $\mathrm{mMCD} / \mathrm{FCD}$, and to identify its predictors. In particular, we wanted to quantify the level of cognitive functioning - expressed as full-scale IQ-scores - in these children, both before and after epilepsy surgery. Furthermore, we aimed to assess whether or not the following clinical factors determined a change in IQ-scores after surgery: histopathological diagnosis, age of onset and at surgery, duration of epilepsy, preoperative high seizure frequency and epileptic encephalopathy, surgery type, and post-operative seizure- and AED status. 


\section{Methods}

All children were included who underwent epilepsy surgery between 2000 and 2012 at the University Medical Center Utrecht, had a histopathological diagnosis of an isolated focal cortical dysplasia or mMCD, and underwent neuropsychological testing.

We reviewed the surgical database for all patients with a reported histological diagnosis that could be compatible with focal cortical dysplasia (e.g. malformation of cortical development, microdysgenesis, dysplasia) and revised the histology according the most recent ILAE classification ${ }^{13}$. Patients with dysplastic lesions in the context of other epileptogenic pathologies (FCD III types e.g. with mesiotemporal sclerosis or tumours) and those with complex or multifocal malformations of cortical development (e.g. polymicrogyria, hemimegalencephaly, tuberous sclerosis complex) were excluded.

Children were neuropsychologically assessed shortly before, one or two years after surgery. A range of tests was used, according to the age and cognitive level of the children, to determine their (full-scale) Intelligence Quotient (IQ). Most frequently used was the Wechsler Intelligence Scale for Children-III (WISC-III) (pre/post $n=17)$, other tests were Wechsler Intelligence Scale for Children Revised (WISC-R) (pre/post $n=5$ ), Wechsler Adult Intelligence Scale (WAIS) (pre/post $n=3 / 4$ ), Snijders-Oomen non-verbal intelligence tests (SON) (pre/post $n=3$ ), Bayley Scales of Infant and Toddler Development - II (BSID-II) (pre/ post $n=6 / 8$ ), Wechsler Preschool and Primary Scale of Intelligence-III (WPPSI-III) (pre/ post $n=3 / 2$ ) and Kaufman Adolescent and Adult Intelligence Test (KAIT) (pre/post $n=1$ ). For children who scored below the lower end of the norms' range, or for whom the ageappropriate tests were too difficult to perform, a developmental quotient (DQ) was calculated, using the ratio mental age / calendar age $\times 100$.

Change in IQ/DQ was defined as post-surgical (one or two years after surgery) minus presurgical IQ/DQ. An IQ or DQ change of 10 points was considered clinically meaningful. Ten IQ points is two-thirds of a standard deviation and three times the standard error of the most used test in this cohort, the WISC-III, and an IQ change of 10 is considered unlikely to be the result of retesting or related to errors inherent to the performed test. Ratio DQ is known to be less stable than normed IQ. For the main analysis, children with IQ and DQ scores were pooled. Separate analyses were performed for patients in whom normed IQ scores were obtained, to address possible influence of incorporating DQ in the analyses. Determinants for IQ/DQ change were additionally studied excluding patients with hemispheric surgery, to rule out this subgroup determines possible associations in the total cohort. We categorized results of neuropsychological testing as normal (IQ > 85), mild delay (IQ 70 -85), moderate delay (IQ 55-70) and severe delay (IQ < 55). In the latter group with severe 
delay were many children in whom norm IQ scores could not be determined. Children with $\mathrm{IQ} / \mathrm{DQ}<70$ are considered to have cognitive impairment.

The following clinical information was collected from the electronic patient file: presence of an MRI lesion compatible with FCD or $\mathrm{mMCD}$, presence of bilateral epileptic encephalopathy in the period prior to surgery, occurrence of generalized tonic clonic seizures, seizure frequency, age at onset of epilepsy, location of seizure focus, location of surgery (multilobar, frontal, temporal, parietal, occipital), age at surgery, type of surgery (hemispheric versus focal), post-surgical seizure freedom (defined as Engel la; completely seizure-free since surgery) and the use of anti-epileptic drugs at latest follow-up and at time of latest neuropsychological assessment. We additionally used the dichotomized seizure outcome "Worthwhile seizure reduction" or not, corresponding with Engel classes 1-3, and defined as more than 50\% reduction in seizure frequency. Information on post-surgical seizure and medication status was completed by means of telephone interview, the time of which was noted as latest follow-up.

Acute postoperative seizures during the first month were not taken into consideration when classifying seizure outcome.

\section{Statistical analysis}

Statistical analysis was performed in SPSS Statistics version 25.

For testing for normality we used Shapiro-Wilk test, and histogram and Q-Q plot. TIQ was normally distributed and T-test (including Levene's test for testing equal variances) was used to compare groups, or ANOVA for the three pathology groups, with Bonferroni correction for post-hoc tests. Change in IQ/DQ, age at surgery, epilepsy onset and duration had non-normal distribution for which we used appropriate non-parametric tests, either Kruskal-Wallis or Mann-Whitney U. Fisher's exact was used test to analyse association between baseline characteristics, cognitive functioning categories and meaningful IQ/DQ change ( $\geq 7$ points), and histology. Kendall Tau- $C$ was used for the association between cognitive functioning categories and meaningful IQ/DQ change and presence of daily seizures, epileptic encephalopathy, type of surgery (focal versus hemispheric), seizure- or AED-freedom in order to account for ordinal ties. Pearson's R or Spearman's rank were used for analysis of correlation. For comparing IQ/DQ before and after surgery related samples paired T-test was used and for categories of cognitive functioning Wilcoxon signed rank test.

\section{Results}

Between 2000 and 2012, 88 patients underwent epilepsy surgery and had a diagnosis of isolated FCD or mMCD according to the ILAE guidelines. Fifty-two were under the age of 18 years at time of surgery. The 42 who underwent cognitive testing at any time, either 
before surgery, after surgery, or both, were included. Of these, the revised pathological diagnosis was mMCD in 10, FCD I in 6, and FCD II in 26 children. For 30 patients normed IQ scores could be calculated with age-appropriate tests both before and after surgery. In five children, only developmental quotient scores could be determined pre- and postsurgery. One child had a normed IQ score pre- and DQ post-surgery. Three patients had no neuropsychological examination prior to surgery, and one not after surgery. Two patients had pre-surgical IQ/DQ scores $<55$, that could not be further quantified, of which one with a post-surgical IQ and one with a DQ score.

Therefore, in 36 patients IQ/DQ scores could be compared between pre and post-surgery tests. In a total of 38 children, categories of functioning could be determined both before and after surgery.

The latest cognitive test was performed two years after surgery in 35 patients, and at one year in 6 patients.

In three patients re-resection was performed within one year and thus before having done their first post-operative neuropsychological evaluation. For these patients post-operative seizure outcome and cognitive function was reported after the second surgery. Baseline functioning was tested prior to first surgery in all cases.

\section{Clinical characteristics}

Clinical characteristics of patients are presented in table 1. Median age at onset and surgery were 2.3 and 10.0 years respectively, with no statistical difference between histological diagnoses. Children with FCD I and $\mathrm{mMCD}$ were more likely to undergo hemispheric surgery $(p=0.004)$ and presented more often with epileptic encephalopathy $(p=0.005)$.

Prior to surgery, 20/39 (51\%) of all tested children had IQ/DQ values $<70$, with a mean score of 66 (distribution of IQ/DQ scores displayed in table 2).

At time of the latest post-operative cognitive assessment, 24 (59\%) were seizure free and 16 (39\%) were also already free of medication. Thirty-five (85\%) children achieved at least worthwhile seizure reduction (Engel 1-3). There was a trend towards less patients with $\mathrm{mMCD}$ diagnoses to be seizure- and medication-free at time of latest post-surgical cognitive testing $(p=0.055)$.

\section{Determinants of pre-surgical cognitive functioning}

All studied determinants of pre-surgical cognitive functioning are shown in table $3 a$. There were significant differences in pre-surgical IQ between the three pathology groups $(p=0.004)$. Patients with FCD I had significantly lower DQ/IQ (post-hoc testing FCD I versus mMCD: $p=0.030$, versus FCD II: $p=0.003$ ). The difference between FCD II and mMCD was not significant and there was no statistical difference when only analysing the children with normed IQ scores. There was no significant correlation between pathology groups and 
Table 1. Baseline characteristics

\begin{tabular}{|c|c|c|c|c|c|c|}
\hline & $\begin{array}{l}\text { Total } \\
n=42\end{array}$ & $\begin{array}{l}\mathrm{mMCD} \\
10(24 \%)\end{array}$ & $\begin{array}{l}\text { FCD total } \\
32(76 \%)\end{array}$ & $\begin{array}{l}\text { FCD I } \\
6(14 \%)\end{array}$ & $\begin{array}{l}\text { FCD II } \\
26(62 \%)\end{array}$ & $\begin{array}{l}\text { p mMCD } \\
\text { vs FCD I } \\
\text { vs FCD II }\end{array}$ \\
\hline Sex, male & $24(57 \%)$ & $4(40 \%)$ & $20(63 \%)$ & $3(50 \%)$ & $17(65 \%)$ & 0.370 \\
\hline $\begin{array}{l}\text { Age at epilepsy onset, } \\
\text { median and range (years) }\end{array}$ & $2.3(0-12.0)$ & $\begin{array}{l}5.9(0.0- \\
10.0)\end{array}$ & $2(0.0-12.0)$ & $1.5(0.0-8.0)$ & $\begin{array}{l}2.0(0.0- \\
12.0)\end{array}$ & 0.424 \\
\hline $\begin{array}{l}\text { Age at surgery, median and } \\
\text { range (years) }\end{array}$ & $\begin{array}{l}10.0(0.2- \\
18.5)\end{array}$ & $\begin{array}{l}11.9(1.0- \\
16.3)\end{array}$ & $\begin{array}{l}9.6(0.2- \\
18.5)\end{array}$ & $\begin{array}{l}4.3(0.6- \\
15.8)\end{array}$ & $\begin{array}{l}10.0(0.2- \\
18.5)\end{array}$ & 0.445 \\
\hline $\begin{array}{l}\text { Duration of epilepsy, } \\
\text { median and range (years) }\end{array}$ & $4.5(0-17.0)$ & $4(1-15)$ & $5.5(0-17)$ & $2.5(1-10)$ & $6(0-17)$ & 0.239 \\
\hline generalized seizures (ever) & $30(71 \%)$ & $4(60 \%)$ & $24(75 \%)$ & $5(83 \%)$ & $19(73 \%)$ & 0.620 \\
\hline generalized seizures (last yr.) & $29(69 \%)$ & $5(50 \%)$ & $24(75 \%)$ & $5(83 \%)$ & $19(73 \%)$ & 0.387 \\
\hline Daily seizures & $31(74 \%)$ & $7(70 \%)$ & $24(75 \%)$ & $5(83 \%)$ & $19(73 \%)$ & 1.000 \\
\hline $\begin{array}{l}\text { Presence of epileptic } \\
\text { encephalopathy pre-surgery }\end{array}$ & $10(24 \%)$ & $4(50 \%)$ & $5(24 \%)$ & $3(50 \%)$ & $2(8 \%)$ & 0.005 \\
\hline \multicolumn{7}{|l|}{ Type of surgery } \\
\hline Hemispheric & $8(19 \%)$ & $2(20 \%)$ & $6(19 \%)$ & $4(67 \%)$ & $2(8 \%)$ & 0.004 \\
\hline Focal & $32(76 \%)$ & $8(80 \%)$ & $28(88 \%)$ & $2(33 \%)$ & $26(100 \%)$ & \\
\hline Frontal & $21(62 \%)$ & $3(38 \%)$ & $18(56 \%)$ & $1(50 \%)$ & $17(71 \%)$ & 0.074 \\
\hline Temporal & $7(21 \%)$ & $3(38 \%)$ & $4(13 \%)$ & $1(50 \%)$ & $3(13 \%)$ & \\
\hline Parietal & $4(12 \%)$ & 0 & $4(13 \%)$ & 0 & $4(17 \%)$ & \\
\hline Occipital & $2(6 \%)$ & $2(25 \%)$ & $2(6 \%)$ & 0 & $2(6 \%)$ & \\
\hline Lateralisation, left sided & $20(48 \%)$ & $6(60 \%)$ & $14(44 \%)$ & $2(33 \%)$ & $12(46 \%)$ & 0.681 \\
\hline MRI-negative lesion & $6(14 \%)$ & $2(20 \%)$ & $4(13 \%)$ & $2(33 \%)$ & $2(8 \%)$ & 0.101 \\
\hline $\begin{array}{l}\text { Re-operations (intra-cranial } \\
\text { surgery) }\end{array}$ & $7(17 \%)$ & $2(20 \%)$ & $5(16 \%)$ & $2(33 \%)$ & $3(12 \%)$ & 0.290 \\
\hline $\begin{array}{l}\text { Engel } 1 \mathrm{~A} \text {, year of latest cog } \\
\text { test }\end{array}$ & $24 / 41(59 \%)$ & $4(40 \%)$ & $20(65 \%)$ & $5(83 \%)$ & $15(60 \%)$ & 0.263 \\
\hline $\begin{array}{l}\text { Engel } 1 \mathrm{~A} \text { drug-free, year of } \\
\text { latest cog test }\end{array}$ & 16/41 (39\%) & $1(10 \%)$ & $15(48 \%)$ & $4(67 \%)$ & $11(44 \%)$ & 0.055 \\
\hline $\begin{array}{l}\text { Engel 1-3 (worthwhile } \\
\text { seizure reduction) }\end{array}$ & $35 / 41(85 \%)$ & $9(90 \%)$ & $26(84 \%)$ & $6(100 \%)$ & $20(80 \%)$ & 0.566 \\
\hline
\end{tabular}

categories of cognitive functioning ( $p=0.097$ ), although it is striking that all children with FCD I had cognitive impairment. Higher pre-surgical cognitive functioning was strongly related to older age at epilepsy onset $(p=0.001)$. Patients with severe cognitive delay were operated earlier in life, but age at surgery was similar for the other categories of cognitive ability. Therefore, in the total group, age at surgery and IQ/DQ score were positively correlated $(p=0.021)$, while there was no association when excluding those with DQ scores, since 6 of $7(86 \%)$ of them had severe delay. In patients with normed IQ scores, lower pre-surgical IQ was related to a longer duration of epilepsy $(p=0.022)$. Almost all patients with $D Q$ scores had severe delay, and these children had shorter epilepsy duration, although differences 
Table 2. cognitive functioning pre and post-surgery

\begin{tabular}{llll}
\hline & Before surgery & after surgery & p \\
\hline Categories cognitive functioning & & & 0.248 \\
\hline severe delay & $8(22 \%)$ & $8(22 \%)$ & $11(31 \%)$ \\
\hline moderate delay & $10(22 \%)$ & $8(22 \%)$ & \\
\hline mild delay & $14(39 \%)$ & $9(25 \%)$ & $\mathbf{0 . 0 1 0}$ \\
\hline normal & $4(11 \%)$ & $70( \pm 20 ; 31-103)$ & $\mathbf{0 . 0 2 9}$ \\
\hline Mean IQ/DQ (std, range) & $66( \pm 18 ; 17-96)$ & $75( \pm 17 ; 49-103)$ & \\
\hline
\end{tabular}

Numbers in this table are based on the 36 patients in whom both pre and post-surgical cognition had been assessed. For 30 of these patients normed IQ scores were available both pre and postoperatively.

in epilepsy duration between the four categories of pre-surgical cognitive functioning did not reach significance $(p=0.112)$

Patients with daily seizures tended to be more often in the lowest categories of cognitive functioning $(p=0.057)$ and there was a trend for lower IQ/DQ in these children $(p=0.052)$. in patients with a clinical presentation compatible with epileptic encephalopathy, there was an overrepresentation of patients with severe cognitive delay $(p=0.017)$ and IQ/DQ was lower, but not significantly so $(p=0.079)$. IQ/DQ was significantly lower in patients who were to undergo hemispheric surgery $(p=0.031)$.

\section{Change in cognitive functioning after surgery}

After surgery, the proportion of children in each of the four cognitive functioning categories did not significantly change, although relatively more had normal IQ after surgery (Table 2). When pooling IQ and DQ scores for all patients with both discrete pre- and post-operative values available $(n=36)$, mean quotients significantly increased from 66 to $70(p=0.010)$. Considering only the children in whom normed IQ scores could be determined $(n=30)$, mean IQ increased from 71 to $75(p=0.029)$.

Whether or not there was a clinically relevant IQ change could be determined in 37 children. Nine of these (24\%) showed an improvement of $\geq 10$ points, 2 (5\%) deteriorated $\geq 10$ points, and $26(70 \%)$ had post-surgical scores within 10 IQ points of their pre-surgical scores.

\section{Determinants of post-surgical cognitive functioning}

All studied possible determinants of post-surgical cognitive functioning are shown in table $3 \mathrm{~b}$. Post-surgical IQ/DQ was strongly correlated with pre-surgical IQ/DQ. No significant correlation was seen between histological subtype and post-operative cognition. The relation between post-surgical cognitive functioning and duration of epilepsy and age at onset and surgery, 


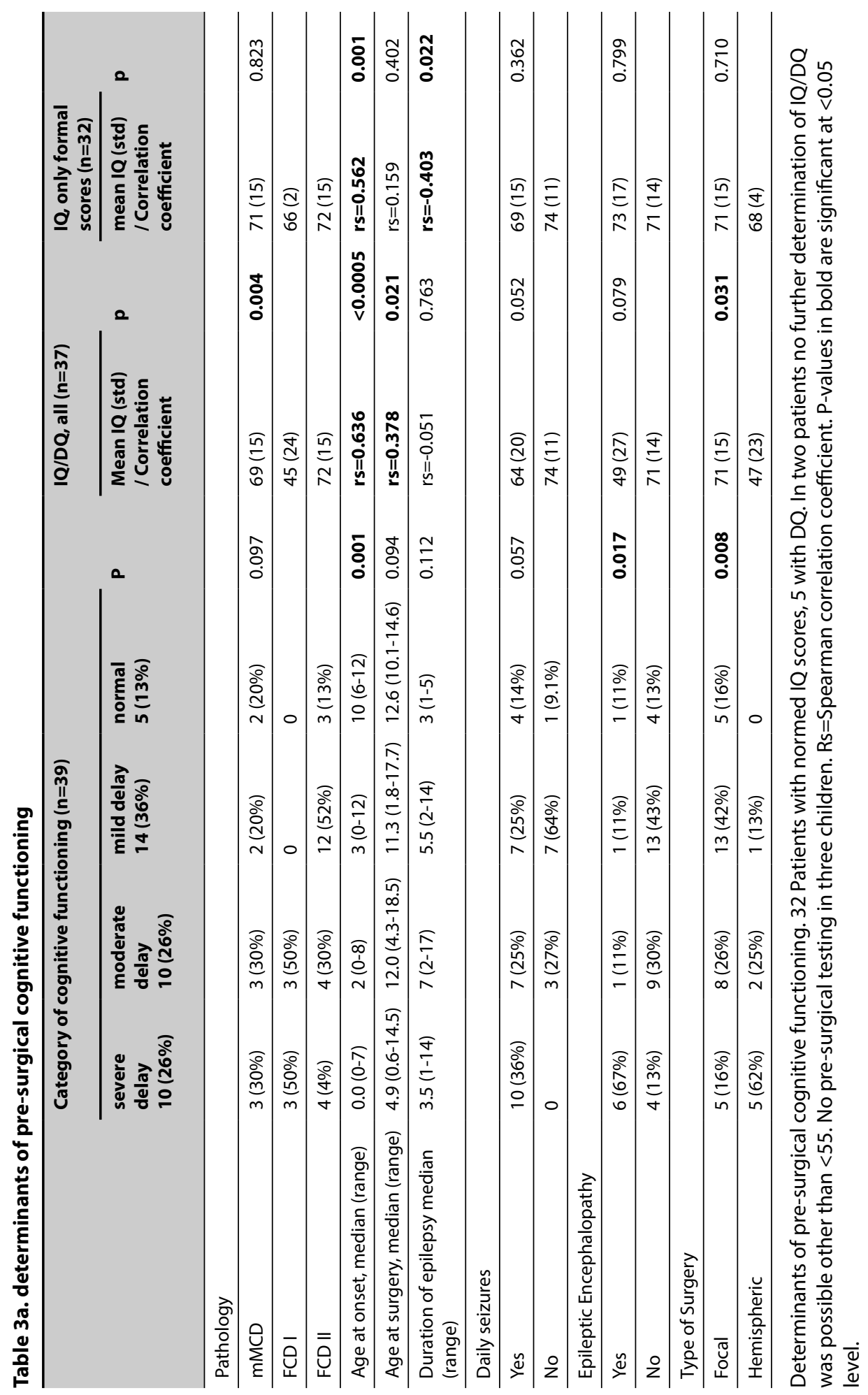




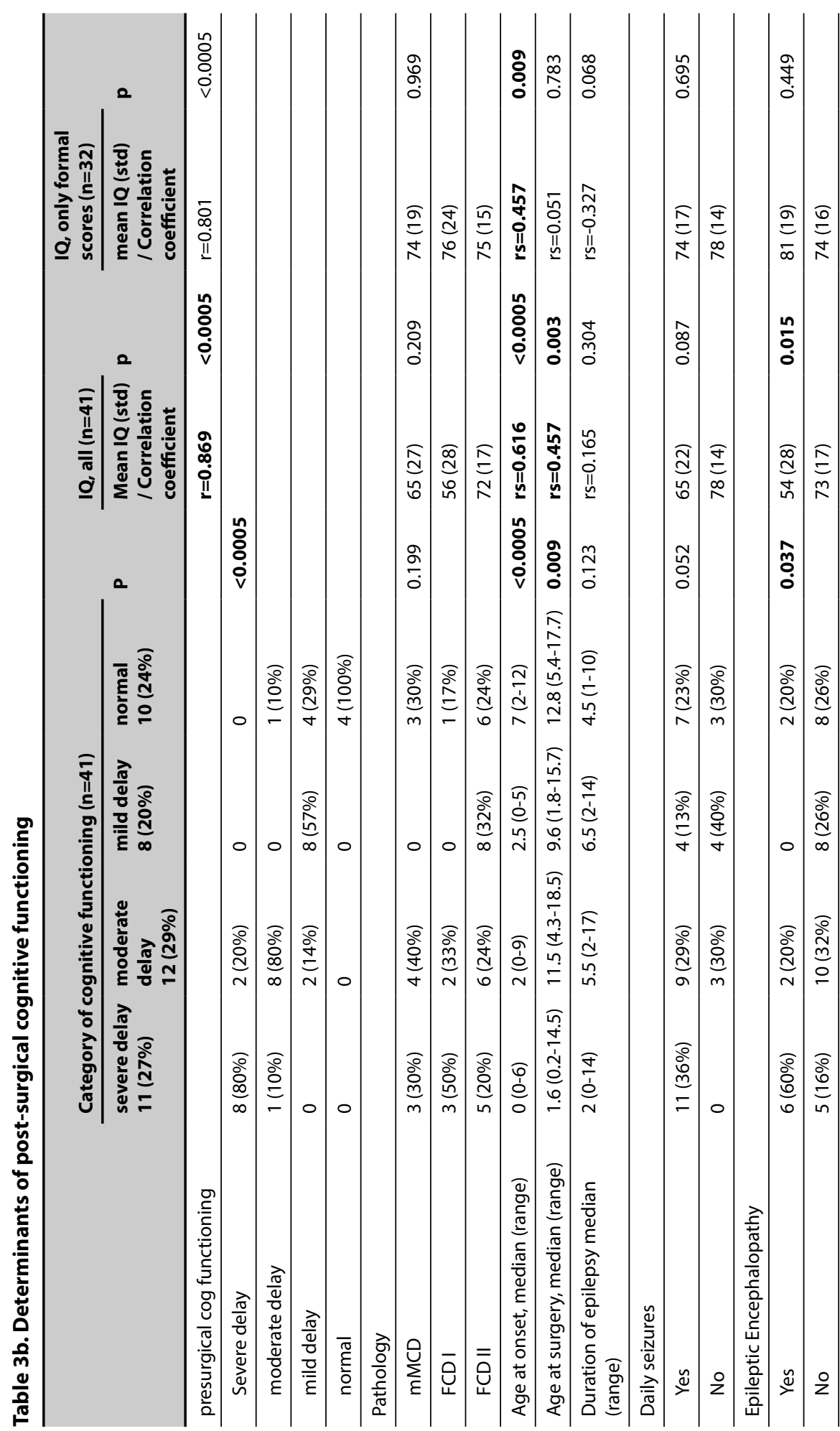




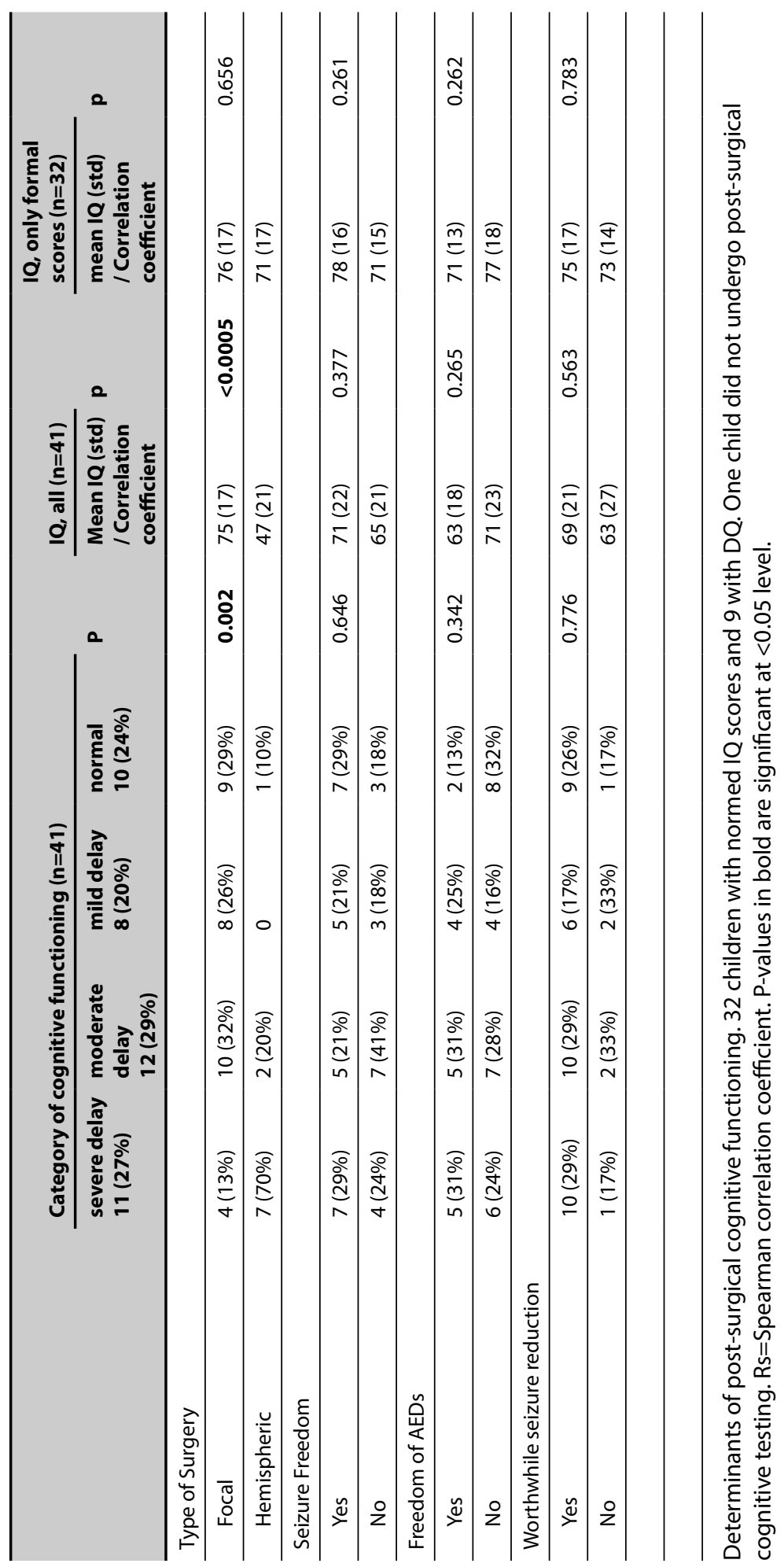


was similar to that found before surgery; younger ages at onset and at surgery were correlated with lower IQ ( $p<0.0005$ and 0.003 respectively). Patients with severe cognitive delay were operated far earlier $(p=0.009)$. Again, after exclusion of the children with only DQ available, there was no association between age at surgery and IQ. Lower post-surgical cognitive scores tended to correlate with longer epilepsy duration $(p=0.068)$.

Patients who suffered from epileptic encephalopathy, and those who underwent hemispheric surgery, were more often in the lowest categories of post-surgical cognitive functioning $(p=0.037$ and $p=0.002)$. IQ/DQ was also significantly lower in these patients $(p=0.015$ and $<0.0005)$, but not when excluding patients with only DQ.

There was no relation between cognitive functioning and freedom of seizures or of antiepileptic drugs.

\section{Determinants of change in cognitive functioning}

Studied determinants of change in cognitive functioning are shown in table $3 c$.

There was no relation between change in cognitive scores and presurgical cognition, epilepsy duration, nor with age at onset or surgery.

Five of eight (63\%) patients with epileptic encephalopathy in whom IQ change could be assessed showed an improvement of $\geq 10$ points, compared to only 4 of 29 (14\%) patients without epileptic encephalopathy $(p=0.017)$. Change in IQ was significantly higher in patients with EE $(p=0.049)$. Children with FCD II diagnoses were more likely to have stable IQ/DQ after surgery and less likely to have an increase of 10 points or more, in comparison to children with mMCD and FCD I diagnoses ( $p=0.024)$.

Change in IQ, and the proportion of patients with a clinically relevant change in IQ, were not different between patients who achieved seizure freedom and those with recurrent seizures, neither did AED discontinuation influence change in IQ.

Analyses were repeated with exclusion of patients who underwent hemispheric surgery; in this subgroup 6 (20\%) had clinically relevant TIQ improvement, 24 (80\%) had stable scores, and no patients deteriorated. In this subgroup of patients, however, there was no significant correlation any more between pre-surgical EE and IQ/DQ change $(p=0.177)$, or between an increase of 10 or more IQ points and presurgical $E E(p=0.094)$. In this subgroup of patients who did not undergo hemispheric surgery, those with IQ improvement of $\geq 10$ points had experienced later age at onset compared to patients with stable IQ $(p=0.005)$. The results of this sub-analysis are shown in supplement table 1. 
Table 3c. Determinants of change in cognitive functioning

\begin{tabular}{|c|c|c|c|c|c|c|}
\hline & \multicolumn{3}{|c|}{ relevant IQ change ( $\geq 10$ IQ points) $(n=37)$} & \multirow[b]{2}{*}{$\mathbf{p}$} & \multirow{2}{*}{$\begin{array}{l}\Delta \mathrm{IQ}(\mathrm{n}=35) \\
\text { median (range) } \\
\text { / Correlation } \\
\text { coefficient }\end{array}$} & \multirow[b]{2}{*}{$\mathbf{p}$} \\
\hline & $\begin{array}{l}\text { deterioration } \\
n=2(5 \%)\end{array}$ & $\begin{array}{l}\text { stable } \\
n=26(70 \%)\end{array}$ & $\begin{array}{l}\text { improvement } \\
n=9(24 \%)\end{array}$ & & & \\
\hline \multicolumn{7}{|l|}{ Pathology } \\
\hline $\mathrm{mMCD}$ & $1(11 \%)$ & $5(56 \%)$ & $3(33 \%)$ & 0.024 & $1(-12-+18)$ & 0.326 \\
\hline FCD I & $1(17 \%)$ & $2(33 \%)$ & $3(50 \%)$ & & $11(-11-+39)$ & \\
\hline FCD II & $0(0 \%)$ & $19(86 \%)$ & $3(14 \%)$ & & $3(-9-+16)$ & \\
\hline \multicolumn{7}{|l|}{$\begin{array}{l}\text { presurgical cog } \\
\text { functioning }\end{array}$} \\
\hline Severe delay & $1(11 \%)$ & $5(56 \%)$ & $3(33 \%)$ & 0.451 & $6.5(-12-+14)$ & 0.715 \\
\hline Moderate delay & $1(10 \%)$ & $8(80 \%)$ & $1(10 \%)$ & & $1(-11-+39)$ & \\
\hline Mild delay & 0 & $11(79 \%)$ & $3(21 \%)$ & & $2.5(-9-+18)$ & \\
\hline Normal & 0 & $2(50 \%)$ & $2(50 \%)$ & & $9(-2-+14)$ & \\
\hline $\begin{array}{l}\text { Presurgical IQ/DQ, } \\
\text { mean (range) }\end{array}$ & $61(54-68)$ & $66(23-96)$ & $65(17-90)$ & 0.908 & $r s=-0.046$ & 0.789 \\
\hline $\begin{array}{l}\text { Age at onset, median } \\
\text { (range) }\end{array}$ & $4(0-8)$ & $2(0-12)$ & $6(0-12)$ & 0.116 & $\mathrm{rs}=0.018$ & 0.919 \\
\hline $\begin{array}{l}\text { Age at surgery, } \\
\text { median (range) }\end{array}$ & $7.7(1.0-14.3)$ & $\begin{array}{l}10.0(1.8- \\
18.5)\end{array}$ & $12.6(0.6-17.7)$ & 0.794 & $r s=-0.006$ & 0.973 \\
\hline $\begin{array}{l}\text { Duration of epilepsy } \\
\text { median (range) }\end{array}$ & $3.5(1-6)$ & $6(2-17)$ & $4(1-10)$ & 0.165 & $r s=-0.121$ & 0.480 \\
\hline \multicolumn{7}{|l|}{ Daily seizures } \\
\hline Yes & $2(7 \%)$ & $18(67 \%)$ & 7 (26\%) & 1.000 & $4(-12-+16)$ & 0.876 \\
\hline No & 0 & $8(80 \%)$ & $2(20 \%)$ & & $2.5(-9-+39)$ & \\
\hline \multicolumn{7}{|l|}{$\begin{array}{l}\text { Epileptic } \\
\text { Encephalopathy }\end{array}$} \\
\hline Yes & $1(13 \%)$ & $2(25 \%)$ & $5(63 \%)$ & 0.017 & $13(-12-+18)$ & 0.049 \\
\hline No & $1(3 \%)$ & $24(83 \%)$ & $4(14 \%)$ & & $2(-11-+39)$ & \\
\hline \multicolumn{7}{|l|}{ Type of Surgery } \\
\hline Focal & 0 & $24(80 \%)$ & $6(20 \%)$ & 1.000 & $3(-9-+39)$ & 0.611 \\
\hline Hemispheric & $2(29 \%)$ & $2(29 \%)$ & $3(43 \%)$ & & $8(-12-+18)$ & \\
\hline \multicolumn{7}{|l|}{ Seizure Freedom } \\
\hline Yes & $2(9 \%)$ & $14(61 \%)$ & $7(30 \%)$ & 0.573 & $7(-12-+39)$ & 0.626 \\
\hline No & 0 & 12 (86\%) & $2(14 \%)$ & & $2(-9-+14)$ & \\
\hline \multicolumn{7}{|l|}{ Freedom of AEDs } \\
\hline Yes & $2(13 \%)$ & $11(73 \%)$ & $2(13 \%)$ & 0.103 & $-2(-12-+16)$ & 0.125 \\
\hline No & 0 & $15(68 \%)$ & 7 (32\%) & & $5(-9-+39)$ & \\
\hline \multicolumn{7}{|l|}{$\begin{array}{l}\text { Worthwhile seizure } \\
\text { reduction }\end{array}$} \\
\hline Yes & $2(6 \%)$ & $21(66 \%)$ & $9(28 \%)$ & 0.528 & $4(-12-+39)$ & 0.723 \\
\hline No & 0 & $5(100 \%)$ & 0 & & $1(-2-+8)$ & \\
\hline
\end{tabular}

$\mathrm{Rs}=$ Spearman correlation coefficient. P-values in bold are significant at $<0.05$ level. 


\section{Discussion}

In our study of cognitive functioning in 42 children who underwent surgery for refractory epilepsy due to mMCD or FCD, 51\% had cognitive impairment prior to surgery. Cognitive functioning was poorer in patients with onset at younger age. Patients with severe cognitive impairment were often operated at very young age and consequently had the shortest epilepsy duration. In patients with IQ/DQ above 55, shorter epilepsy duration was related to better presurgical cognitive functioning. FCD I was infrequently diagnosed but these patients all had cognitive impairment and mean IQ/DQ was significantly lower than that in $\mathrm{mMCD}$ and FCD II.

We found an overall modest but statistically significant improvement of 4 mean IQ/DQ points, within 1 to 2 years after surgery. Relevant improvement of $\geq 10$ IQ/DQ points was seen in $24 \%$ following surgery, while cognitive functioning was stable in $70 \%$ and deteriorated in $5 \%$. Only a diagnosis of epileptic encephalopathy predicted cognitive improvement after surgery. Post-surgical cognitive functioning was strongly related to pre-surgical functioning and had similar associations with clinical characteristics as pre-surgical functioning. There was no association between postoperative cognitive scores and seizure or medication status.

Other mMCD/FCD studies have found a similar prevalence of cognitive impairment ${ }^{27,28}$. Seizure activity and consequent network disturbances, the underlying pathology and use of anti-epileptic drugs are all likely to contribute to cognitive impairment.

In a rat model for FCD, in which developmental abnormalities were caused by injection of methyloaxymethanol acetate and consequently frequent seizures were induced in one group, researchers found no relation between cognition in adolescence and history of early-life seizures and concluded that the cortical malformations were the substrate for cognitive impairment. However, these mice with cortical malformations had significantly lower brain weights compared to a control group that received saline injections in-utero, suggesting methyloaxymethanol acetate injection causes more widespread abnormalities than those seen in human patients with $\mathrm{FCD}^{79}$.

Despite the circumscript nature of dysplastic lesions in most patients with FCD or mMCD, widespread network disruption can be observed, even when their seizures remain focal. There is evidence of loss of optimal, small-world, network configuration and abnormal diffusivity and decreased anisotropy in white matter distant from, and even contralateral to, the lesion ${ }^{30,31,80,81}$. This results in cognitive deficits not directly associated with the location of a lesion. Following remission of epilepsy, both normalization of functional network organization and recovery of white matter bundle fractional anisotropy may be seen ${ }^{31}$. Also AED drug load is associated with disturbed network parameters ${ }^{80}$. Drugs may have a generalized suppressive effect with alteration of neuronal excitability ${ }^{80,82}$. Consequently, 
many common AEDs are associated with cognitive problems. After diagnosis of epilepsy there is more often cognitive decline in patients with higher AED levels ${ }^{83}$. In addition, in a randomized blinded placebo-controlled trial in seizure free patients cognitive function improved if AED treatment was discontinued ${ }^{84}$. Likewise, a retrospective study in children who underwent epilepsy surgery showed improved post-surgical IQ and higher IQ change with reduction of $A E D s^{53}$.

In agreement with previous studies we found a relation between poorer cognition and younger age at onset ${ }^{27,46,55-62}$. Pre-surgical impaired cognition was associated with longer duration of epilepsy, but only when excluding children with only DQ, of whom 6/7 (86\%) had expedited surgical intervention due to catastrophic epilepsy syndromes. Kimura et al. found a similar relation between cognition and epilepsy duration when children were stratified by age of onset, but not when analysing the complete cohort ${ }^{27}$. This is in agreement with another study that included only children that were operated before the age of three years ${ }^{63}$. With impairment of brain function due to seizure activity, longer exposure to epileptic activity would reasonably increase the gap in cognitive function with normal developing peers.

A critical period in cognitive development during the first years of life has been suggested which lays the foundation for subsequent development of higher-order skill ${ }^{60}$. Disruption during sensitive periods could result in more pronounced cognitive deficits later in life ${ }^{85}$. On the other hand, both lower IQ and an earlier epilepsy onset could be the consequence of a more severe underlying epileptogenic pathology, rather than being causally related ${ }^{61}$.

Patients with severe cognitive delay were operated at younger age, but when excluding patients with only DQ-of whom 6/7 (86\%) had severe delay-we did not observe a relation between age at surgery and pre or post-surgical cognitive ability. In a cohort consisting only of patients undergoing hemispherectomies, worse cognition was related to surgery at younger age and shorter epilepsy duration ${ }^{86}$.

Presurgical cognition was lower in patients with FCD I compared to mMCD or FCD II. A possible explanation is that FCD I lesions are typically more widespread - with more frequent hemispheric surgery in our cohort - and these patients presented more often with epileptic encephalopathy. The latter could also explain why differences in cognition between histological subtypes were not significant after surgery; we observed greater improvement in cognition in patients with previous encephalopathy and more children with clinically relevant IQ improvement among those with $\mathrm{mMCD}$ and FCD I histological subtypes compared to FCD II. Previous studies did not find a relation between cognition and histological subtype ${ }^{27,42}$, however, these groups used the 2002 Palmini histological classification scheme, what can lead to discrepancies in classification, especially in more subtle abnormalities. 
A large share of patients in our cohort showed improvement in cognitive level after surgery. Studies showed full-scale IQ to have high stability and reliability with test-retest coefficients exceeding 0.9. Large studies, including around 600 children evaluated WISC-III (the most used test in our cohort) for special education eligibility, found full-scale IQ to decrease - 0.4 in a mean interval just under three years ${ }^{87,88}$. The average 4 point improvement in full-scale IQ on a group level we found is not likely due to retest effects, or retesting at older age since tests have been proven highly stable and are normed according to age. Although presurgical cognition has consistently been shown to be the strongest predictor of post-surgical functioning, as confirmed in our study, it does not necessarily relate to the change in IQ after surgery. For this outcome measure, the presence of pre-surgical epileptic encephalopathy was the only predictive factor in our study. This finding is in agreement with the concept of epileptic encephalopathy, in which epileptic activity by itself affects cognition more than what can be explained by the underlying pathology alone ${ }^{3}$.

We did not observe an association between postoperative cognitive outcome and seizure status, although several studies have reported cognitive improvement to be dependent of seizure freedom ${ }^{49-51}$, with more pronounced improvement after discontinuation of medication as well ${ }^{51}$. These studies had a follow-up of a minimum of 4 years, up to 21 years after surgery. Although we observed overall improvement in cognition, it is likely that a longer follow-up period than two years is required to acknowledge the true effect of seizure freedom and medication policies on cognitive development following epilepsy surgery.

Our study has a number of limitations, besides those inherent to retrospective collection of clinical data and limited follow-up duration for cognition. Our moderate sample size precludes multivariate analyses and correction for possible interactions.

Environmental factors were not accounted for in this study. In a cohort of operated children with epilepsy of diverse aetiology, there was more improvement in cognition in children of parents with higher education, but only in those who became seizure free. Although this association was significant, the effect was small. In the same study there was no relation between parental education and pre-surgical IQ ${ }^{52}$. A large twin-study in a healthy British population showed the effect of genetics on intelligence to be similar among low and high socioeconomic status families ${ }^{89}$.

Despite its limitations, the current study has enabled the assessment of clinically relevant determinants of cognitive outcome in a relatively homogeneous cohort of children with $\mathrm{mMCD} / \mathrm{FCD}$ histopathological diagnoses revised following the most recent ILAE guidelines ${ }^{13}$. 


\section{Conclusion}

In children with mMCD and FCD, pre-operative cognitive functioning is poorer when epilepsy onset is at younger age and duration of epilepsy is longer. Children with severe cognitive delay typically present with catastrophic epilepsy, that warrants an aggressive therapeutic strategy and surgical intervention at very young age.

Surgical therapy in children with refractory epilepsy due to mMCD or FCD not only is likely to result in complete and continuous seizure freedom, but also improves cognitive function in a substantial proportion of children, independent of histological subtype. 
Supplement table 1. Determinants of change in cognitive functioning, excluding children with hemispheric surgery

\begin{tabular}{|c|c|c|c|c|c|c|}
\hline & \multicolumn{3}{|c|}{ relevant IQ change ( $\geq 10$ IQ points) $(n=30)$} & \multirow[b]{2}{*}{$\mathbf{p}$} & \multirow{2}{*}{$\begin{array}{l}\Delta \mathrm{IQ}(\mathrm{n}=29) \\
\text { median (range) } \\
\text { / Correlation } \\
\text { coefficient }\end{array}$} & \multirow[b]{2}{*}{$\mathbf{p}$} \\
\hline & $\begin{array}{l}\text { deterioration } \\
n=0\end{array}$ & $\begin{array}{l}\text { stable } \\
n=24(80 \%)\end{array}$ & $\begin{array}{l}\text { improvement } \\
n=6(20 \%)\end{array}$ & & & \\
\hline \multicolumn{7}{|l|}{ Pathology } \\
\hline $\mathrm{mMCD}$ & 0 & $4(67 \%)$ & $2(33 \%)$ & 0.187 & $0(-9-+13)$ & 0.400 \\
\hline FCD I & 0 & $1(50 \%)$ & $1(50 \%)$ & & $21(3-+39)$ & \\
\hline FCD II & 0 & $19(86 \%)$ & $3(14 \%)$ & & $3(-9-+16)$ & \\
\hline \multicolumn{7}{|l|}{$\begin{array}{l}\text { presurgical cog } \\
\text { functioning }\end{array}$} \\
\hline Severe delay & 0 & $4(80 \%)$ & $1(20 \%)$ & 0.453 & $3.5(-1-+8)$ & 0.720 \\
\hline Moderate delay & 0 & $7(88 \%)$ & $1(13 \%)$ & & $1.5(-2-+39)$ & \\
\hline Mild delay & 0 & $11(85 \%)$ & $2(15 \%)$ & & $1(-9-+16)$ & \\
\hline Normal & 0 & $2(50 \%)$ & $2(50 \%)$ & & $9(-2-+14)$ & \\
\hline $\begin{array}{l}\text { Presurgical IQ/DQ, } \\
\text { mean (range) }\end{array}$ & - & $68(46-96)$ & $79(64-90)$ & 0.101 & $r s=0.060$ & 0.757 \\
\hline $\begin{array}{l}\text { Age at onset, } \\
\text { median (range) }\end{array}$ & - & $2.25(0-12)$ & $7.5(3-12)$ & 0.005 & $r s=0.132$ & 0.494 \\
\hline $\begin{array}{l}\text { Age at surgery, } \\
\text { median (range) }\end{array}$ & - & $\begin{array}{l}10.9(1.8- \\
18.5)\end{array}$ & 13.1 (11.4-17.7) & 0.116 & $r s=-0.204$ & 0.288 \\
\hline $\begin{array}{l}\text { Duration of } \\
\text { epilepsy median } \\
\text { (range) }\end{array}$ & - & $6(2-17)$ & $5.5(1-10)$ & 0.631 & $r s=--0.15$ & 0.938 \\
\hline \multicolumn{7}{|l|}{ Daily seizures } \\
\hline Yes & 0 & $16(76 \%)$ & $5(24 \%)$ & 0.637 & $4(-9-+16)$ & 0.764 \\
\hline No & 0 & $8(89 \%)$ & $1(11 \%)$ & & $1(-9-+39)$ & \\
\hline \multicolumn{7}{|l|}{$\begin{array}{l}\text { Epileptic } \\
\text { Encephalopathy }\end{array}$} \\
\hline Yes & 0 & $1(33 \%)$ & $2(67 \%)$ & 0.094 & $10.5(8-+13)$ & 0.177 \\
\hline No & 0 & $23(85 \%)$ & $4(15 \%)$ & & $2(-9-+39)$ & \\
\hline \multicolumn{7}{|l|}{ Seizure Freedom } \\
\hline Yes & 0 & $13(72 \%)$ & $5(28 \%)$ & 0.358 & $5(-9-+39)$ & 0.492 \\
\hline No & 0 & $11(92 \%)$ & $1(8 \%)$ & & $1(-9-+8)$ & \\
\hline \multicolumn{7}{|l|}{ Freedom of AEDs } \\
\hline Yes & 0 & 10 (91\%) & $1(9 \%)$ & 0.372 & $-2(-9-+16)$ & 0.238 \\
\hline No & 0 & $14(74 \%)$ & $5(26 \%)$ & & $4.5(-9-+39)$ & \\
\hline \multicolumn{7}{|l|}{$\begin{array}{l}\text { Worthwhile seizure } \\
\text { reduction }\end{array}$} \\
\hline Yes & 0 & $19(76 \%)$ & $6(24 \%)$ & 0.553 & $3.5(-9-+39)$ & 0.845 \\
\hline No & 0 & $5(100 \%)$ & 0 & & $1(-2-+8)$ & \\
\hline
\end{tabular}

$\mathrm{Rs}=$ Spearman correlation coefficient. P-values in bold are significant at $<0.05$ level. 



\section{SEVEN TESLA MRI IMPAOVES DETECTION OF FOCAL CORTICAL DYSPLASIA IN PATIENTS WITH REFRACTOAY FOCAL EPILEPSY}

Tim J. Veersema, Cyrille H. Ferrier, Pieter van Eijsden, Peter H. Gosselaar Eleonora Aronica, Fredy Visser, Jaco M. Zwanenburg, Gerard A.P. de Kort Jeroen Hendrikse, Peter R. Luijten, Kees P.J. Braun 
Objective: The aim of this study is to determine if the use of 7 tesla (T) MRI in clinical practice leads to higher detection rates of focal cortical dysplasias in possible candidates for epilepsy surgery.

Methods: In our center patients are referred for 7T MRI if lesional focal epilepsy is suspected, but no abnormalities are detected at one or more previous, sufficient quality lower field MRI scans, acquired with a dedicated epilepsy protocol, or when concealed pathology is suspected in combination with MR-visible mesiotemporal sclerosis-dual pathology. We assessed 40 epilepsy patients who underwent 7T MRI for pre-surgical evaluation, and whose scans (both 7T and lower field) were discussed during multidisciplinary epilepsy surgery meetings that included a dedicated epilepsy neuroradiologist. We compared the conclusions of the multidisciplinary visual assessments of 7T and lower field MRI scans.

Results: In our series of 40 patients, multidisciplinary evaluation of 7TMRI identified additional lesions not seen on lower-field MRI in nine patients (23\%). These findings were guiding in surgical planning. So far six of them underwent surgery, with histological confirmation of focal cortical dysplasia or mild malformation of cortical development.

Significance: 7T MRI improves detection of subtle focal cortical dysplasia and mild malformations of cortical development in patients with intractable epilepsy and may therefore contribute to identification of surgical candidates and complete resection of the epileptogenic lesion, and thus to postoperative seizure freedom. 


\section{Introduction}

In patients with pharmacoresistant focal epilepsy, resection of the epileptogenic zone can be considered. Focal cortical dysplasia (FCD) is the most frequent etiology (42\%) in patients under 18 years of age selected for surgery. In adults FCD is the third most common etiology (13\%) after hippocampal sclerosis (43\%) and tumors $(30 \%)^{70}$.

Focal cortical dysplasias are a subgroup of circumscript malformations of cortical development (MCDs), and are characterized by abnormal migration, maturation, and differentiation of neurons ${ }^{13}$. Onset of epilepsy occurs typically before the age of twelve years and roughly $76 \%$ of patients have medically refractory seizures ${ }^{90}$. Resective surgery can be highly effective, with reported seizure freedom rates ranging from 33 to $80 \%$ in mixed FCD type cohorts of patients operated in the last two decades $28,36-38,40,70,72,75$. In a large study of 211 patients with FCD ILAE type I and II operated 1998-2010, 65\% remained seizure free (Engel class I) up to latest follow-up (2-12 years) ${ }^{37}$. Seizure freedom rate markedly increases in case of complete resection $33,36,37,43,91$, averaging $77 \%$ in a literature review by Lerner and colleagues ${ }^{70}$. Appropriate detection and delineation of a FCD on MRI contributes to identification of surgical candidates and complete resection of the epileptogenic lesion, and thus to postoperative seizure freedom ${ }^{66-68}$.

However, the structural abnormalities in FCD are often very subtle and may escape detection on conventional structural neuroimaging. This makes FCD the most common pathology (45-51\%) in patients who undergo epilepsy surgery for focal refractory epilepsy and have a "non-lesional MRI"70,92. In a review of surgical series from 2000 to 2008, 37\% of FCD type I lesions and $15 \%$ of type FCD type II lesions were not detected with 1.5 or $3 \mathrm{~T} \mathrm{MRI}^{70}$. Detection seems even more problematic for the most subtle architectural abnormalities in the FCD spectrum, classified as mild malformations of cortical development (mMCD), of which $58 \%$ were not visible on MRI in a series published in $2008^{42}$.

Studies comparing 1.5 and 3 tesla (T) MRI in epilepsy surgery candidates have shown that imaging at 3T can identify FCDs that escaped detection at $1.5 T^{93-96}$. In a cohort of patients with FCD type II, 3T MRI showed a lesion in $20 \%$ of patients with non-lesional $1.5 T^{96}$.

We hypothesize that a similar effect can be gained from 7T, by virtue of increased signalto-noise ratio, allowing higher resolution and contrast ${ }^{97}$. Dysplastic lesions previously detected using lower field MRI are reported to be detected with more confidence and visualized in greater detail at $7 \mathrm{~T}^{98}$. One previous study reported an improved detection rate of 7T MRI compared to lower field MRI, although half of the patients in whom a new lesion was detected at $7 \mathrm{~T}$ underwent previous scanning only at $1.5 \mathrm{~T}$, and not at the currently considered optimal field strength of $3 \mathrm{~T}$ in pre-surgical evaluation ${ }^{99}$.

In our study we aim for verification of the clinical value in a larger group of patients, of whom most underwent previous 3T MRI, and using a full 3D scanning protocol with submillimeter resolutions in FLAIR, $T_{1}, T_{2}$ and $T_{2}{ }^{*}$-weighted, and white matter suppression 
sequences. We wanted to determine if the use of 7T MRI in clinical practice led to higher detection rates of focal cortical dysplasia in candidates for epilepsy surgery, and how this influenced surgical decision making.

\section{Materials and methods}

\section{Patients}

The University Medical Center Utrecht is a tertiary referral center for patients with refractory epilepsy and for epilepsy surgery. A multidisciplinary team consisting of neurologists, neurophysiologists, neurosurgeons, neuroradiologists, nuclear medicine physicians, and neuropsychologists from different centers, evaluates surgical candidacy of each patient, determines the type and order of ancillary investigations, and decides on the selection or rejection for surgery and on surgical strategy during epilepsy surgery meetings (ESMs). Patients are referred for 7T MRI if lesional focal epilepsy is suspected but no abnormalities are detected at one or more previous, sufficient quality MRI scans, acquired with a dedicated epilepsy protocol (3T in most cases) ${ }^{100}$, or when concealed pathology is suspected in combination with MR-visible mesiotemporal sclerosis (dual pathology).

For this series we consecutively included 40 patients who underwent 7T MRI for pre-surgical evaluation between March 2009 and February 2016, and whose scans (both 7T and lower field scans) were discussed in the ESMs. Two young patients, age 7 and 8 years, scanned in this period were excluded from analysis. In one the examination had to be stopped because of inconsolable fear. In the other severe movement artefacts in all sequences made accurate assessment impossible. In the 40 included patients at least one sequence was of sufficient quality for reliable visible assessment, although some degree of movement artefacts in one or more sequences was common, especially with pediatric patients. For the review and analysis of patient data institutional ethical board approval was acquired. All patients have given informed consent for the 7T MRI examination.

\section{MRI acquisition}

7T MRI Scans were performed on a Phillips Achieva 7T MRI system (Philips Healthcare, Best, the Netherlands) with a 16 channel receive coil or, after May 2011, a 32-channel receive head coil combined with dual channel transmit coil (Nova Medical, Wilmington, MA, USA). From mid-2015 on, dielectric pads, containing calcium titanate (Leiden University Medical Center, Leiden, the Netherlands) were routinely used to reduce artefacts and signal loss in the temporal regions ${ }^{101}$. Pads were placed to the sides of the subjects' head. Patients all used ear plugs to protect from acoustical noise.

In our center 3T MRI scans were performed with 3T Philips Achieva with eight-channel sensitivity encoding (SENSE) head coil. The 3T is the default machine for epilepsy patients; 
only on specific indication (e.g., vagal nerve stimulator in situ), and in the past, 1.5T was performed, on a Phillips Achieva 8ch or Philips Ingenia dStream.

\section{Scan protocols}

The 7T scanning protocol consists of $T_{1^{\prime}} T_{2^{\prime}}$ FLAIR, and $T_{2}{ }^{*}$-weighted sequences, and a gradient echo white matter suppression (WMS) sequence, all with $0.5-0.8 \mathrm{~mm}$ isotropic resolution with $50 \%$ overlap of slices; acquisition time was circa 45 minutes. Since 2008 there have been minor adjustments in the protocol, mainly the addition of the WMS sequence and modification of the $T_{2}{ }^{*}$ echo times. Parameters of our 1.5, 3, and 7T MRI sequences performed in our center are described in supplementary Table 1. In case of severe motion artefacts, precluding reliable assessment, sequences are repeated to generate at least $T_{1^{\prime}} T_{2}$ and/or FLAIR sequences of sufficient quality, at the cost of $T_{2}^{*}$ and WMS sequences.

All sequences are routinely reformatted in $0.5 \mathrm{~mm}$ (interpolated) multiplanar reconstructions to have axial, coronal, and sagittal images for all sequences, allowing for optimal appreciation of cortical architecture. This protocol covers all sequences recommended in various guidelines for the detection of epileptogenic lesions ${ }^{100}$.

\section{MRI assessment}

MRIs were routinely reviewed (prior to the ESM) by means of visual analysis on a highresolution work station, by a dedicated epilepsy radiologist who takes part in the ESMs, and who also revised all scans performed outside the UMCU. During bi-monthly multidisciplinary meetings, patients' full histories, EEGs, and results of other investigations are discussed. MRI scans are shown on a projector screen in the presence of a neuroradiologist and collectively re-assessed by the entire ESM team. The resulting conclusion is taken as final, and used in this study. Scans were sometimes reviewed on more than one occasion. The reported collective assessment of the lower field MRI always took place prior to the meeting during which the 7T MRI was evaluated.

A radiological diagnosis of possible FCD was made in case of presence of distinctive imaging features ${ }^{102}$, most notably blurring of the gray-white matter border and transmantle sign. Hippocampal atrophy in combination with signal changes was indicative of mesiotemporal sclerosis $^{103}$. Covert dual pathology was suspected if the clinical presentation could not be explained by mesiotemporal sclerosis alone. MRI scans were considered non-lesional if no possibly epileptogenic lesions were seen. Patients with aspecific, or radiological findings strongly divergent from patient and electrophysiological characteristics, were considered MRI-negative, e.g., arachnoid cysts, age-related or gliotic white matter lesions, and enlarged perivascular spaces. No post-processing techniques were used or evaluated for the purpose of this study. 


\section{Surgery}

All surgical procedures were carried out in the University Medical Center Utrecht by two neurosurgeons with expertise in epilepsy surgery. All patients in this series were operated either after chronic electrocorticography (ECOG) or with guidance of acute (intra-operative) ECOG. The surgeons strive to resect tissues en-bloc as much as possible, to allow optimal histopathological examination.

\section{Histopathology}

Resected brain tissue was fixed in $10 \%$ buffered formalin and embedded in paraffin. Paraffinembedded tissue was sectioned at $5 \mu \mathrm{m}$ and used for histology and immunohistochemistry. Sections were processed for hematoxylin eosin staining, as well as for immunohistochemical staining for a number of neuronal and glial markers. Surgical samples were evaluated and classified according to the International League Against Epilepsy (ILAE) guidelines ${ }^{13,104}$.

\section{Results}

\section{Patients characteristics, ancillary tests, and surgical procedures}

Included in this study were 40 patients in whom 7T MRI was performed on clinical indication and whose scans were assessed in multidisciplinary ESMs. Age at time of 7T scan was 7-48 years (median 18).

Thirty-five (88\%) were previously scanned at $3 \mathrm{~T}$, four (10\%) had at least a $1.5 \mathrm{~T}$, and one previously had a 1.0T MRI. Eighteen patients (43\%) underwent both 3T and a 1.5/1/0.5T MRI. Other ancillary tests performed were FDG-PET (in 38, 95\%) and magnetoencephalography (in 29, 73\%). So far, single positron CT (SPECT) has been performed in 15 patients, including one failed examination (38\%) (ictal subtraction with MRI co-registration: $n=12$ ). Three patients were still awaiting SPECT when collecting study data.

Thirteen patients (33\%) underwent resective surgery, all guided by ECOG (chronic grid registration $n=10$, chronic with grid and depth electrodes $n=1$, acute grid registration $n=2$ ). At a follow up ranging 1 month - 5 years (mean 17, median 9 months), 11/12 (91\%) patients are seizure free (Engel score $1 A, n=10$; Engel 1D, $n=1$ ). One patient is scored Engel $4 B$ at 6 months (one patient has not yet been evaluated postoperatively).

Seven patients (18\%) await invasive EEG monitoring. Twelve (30\%) patients were rejected for resective surgery, of whom one underwent multiple subpial transections in the motor cortex and one received a vagal nerve stimulator. Surgery is postponed in one patient with likely FCD because of unexpected and prolonged seizure freedom. In three patients (8\%) the pre-surgical evaluation was stopped, because of expected unacceptable motor deficit, or a significant decrease in burden of disease. Four (10\%) patients are still under evaluation, and await non-invasive tests: SPECT $(n=2)$ and PMRI-EEG $(n=2)$. 


\section{Findings of 7T MRI}

Table 1 provides an overview of 7T and lower-field MRI findings in all patients, as well as the consequent treatment strategies and histological diagnoses, if available.

Table 2 summarizes the findings of relevant ancillary tests in patients with new findings at 7T (patients 1-9, table 2), and those patients who underwent surgery following a clinically indicated 7T without newly identified epileptogenic lesions (patients 10-16, Table 2). A table of all 40 patients is provided as supplementary table 2 .

Thirty-eight patients underwent 7T MRI because lower field MRI was interpreted as showing no relevant epilepsy-related lesions. In eight of those, a focal abnormality, suspect of FCD could be identified on 7T. Six of them have been operated, with histology confirming FCD type Ila in two, FCD IIb in one, mild malformation of cortical development (mMCD) type 2 in two and mild malformation of cortical development with oligodendroglial hyperplasia in one case. (Exemplary cases in figures 1-3). In patient 5 mMCD type 2 was diagnosed, however close relation to language areas precluded en-bloc resection of the complete MRI abnormality ${ }^{104}$. Possibly deeper tissues would have been compatible with FCD I or II types (figure 3). In another patient FCD is also suspected, but surgery with acute ECOG is postponed due to current seizure freedom. One patient is still under evaluation. All patients with new lesions had previous good quality $3 \mathrm{~T}$ MRI scans.

Two patients underwent 7T MRI because of clinical suspicion of dual pathology in combination with mesiotemporal sclerosis (MTS) visualized at 3T MRI. In one patient (patient 10, Table 2) with left-sided MTS and associated dysplasia in the temporal pole there was a clinical and electrophysiological suspicion of an additional contralateral (right-sided) seizure onset.
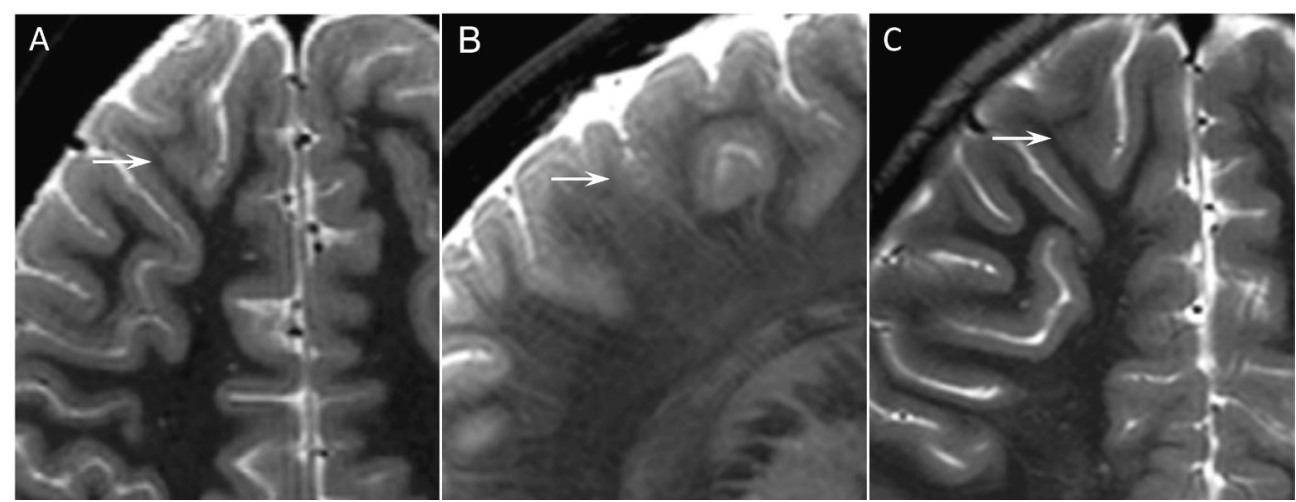

Figure 1. Transverse (A) and sagittal 7T $T_{2}(\mathrm{~B})$, Transverse $3 \mathrm{~T} T_{2}(\mathrm{C})$.

Patient no. 2. $\widehat{\delta}, 7 y \mathrm{rs}$, focal impaired awareness seizures. 7T MRI showed blurring of grey-white matter junction suspect for FCD. The lesion was only retrospectively identified on $3 \mathrm{~T}$, where thick slices ( $4 \mathrm{~mm}+1 \mathrm{~mm}$ gap) lead to many false mimics from partial-volume effects of adjacent gyri. Lesionectomy in the medial frontal gyrus was performed. Histology confirmed FCD ILAE type lla. 


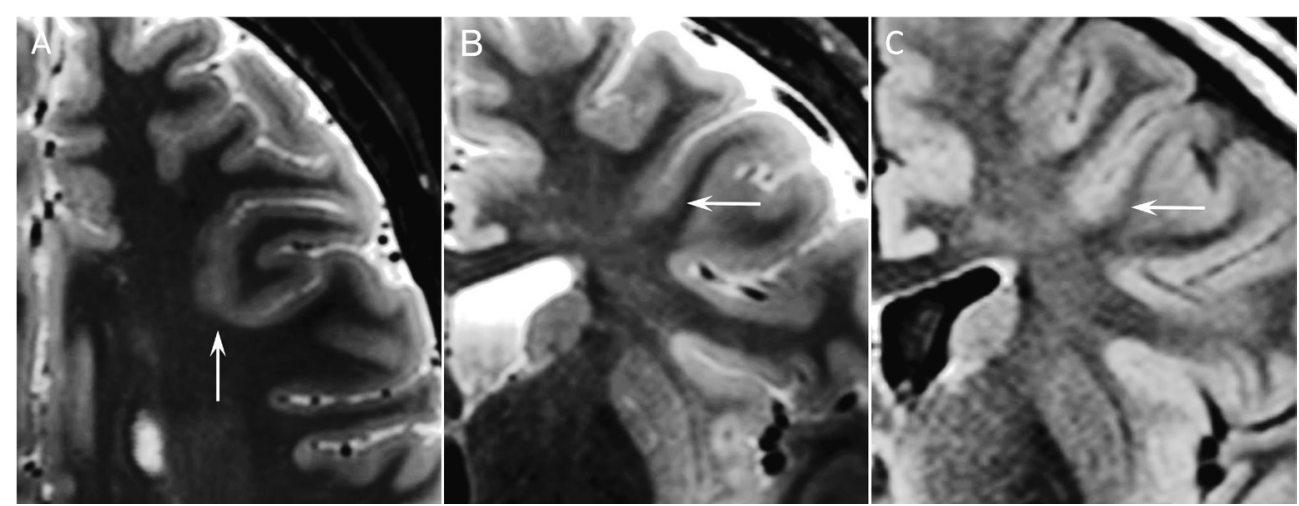

Figure 2. Transverse (A) and coronal 7T $T_{2}(B), 3 T$ FLAIR (C).

Patient no. 6. $\delta^{\lambda}, 14 \mathrm{yrs}$., focal impaired awareness seizures. At 7T blurring of grey-white matter junction and cortical thickening compatible with FCD. In retrospect also recognizable on 3T but initially overlooked due to many (similar looking) partial-volume effects and signal variation in adjacent gyri. ECOG registration plus depth electrodes in the MRI lesion was performed followed by lesionectomy of the bottom of a sulcus in the medial frontal gyrus. Histology confirmed FCD type Ilb.
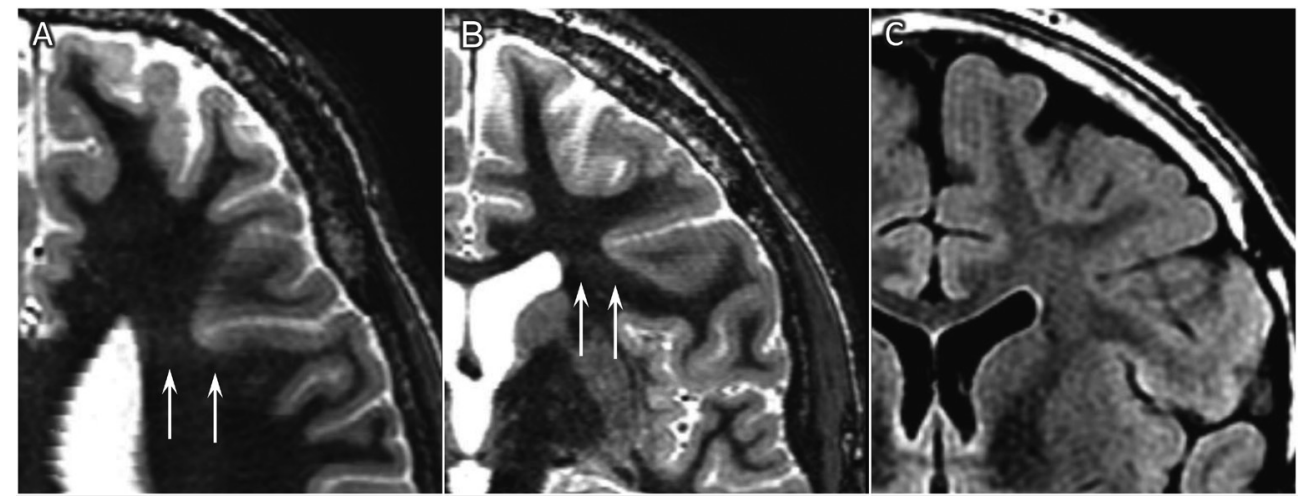

Figure 3. Transverse (A) and coronal 7T white matter suppression sequence (B), coronal 3T FLAIR (C).

Patient no. 5. 0 , 21yrs., focal impaired awareness seizures. On 7T WMS images very subtle white matter signal changes adjoining a deep sulcus (resembling subtle transmantle sign) in a region with a dense gyral pattern, suggestive of subtle cortical dysplasia. No abnormalities had been identified on 3T images. Histology was classified as MMCD type 2, however due to close relation to language areas en-bloc resection of the complete MRI abnormality was not possible and deeper tissues would have been compatible with FCD I or II types.

7T MRI did not indicate the presence of such a lesion and a left-sided anterior temporal lobectomy plus amygdalahipocampectomy was performed. Histopathology confirmed MTS + FCD Illa.

In the other patient (patient 9, Table 2) 3T MRI suggested MTS, but semiology and electrophysiology indicated temporal neocortical pathology. 7T MRI revealed an abnormal 
Table 1. Epilepsy surgery meeting conclusions in patients with non-lesional or unclear lower field MRI.

\begin{tabular}{|c|c|c|c|}
\hline $\begin{array}{l}\text { ESM conclusion } \\
\text { based on lower } \\
\text { field MRI }\end{array}$ & $\begin{array}{l}\text { ESM conclusion } \\
\text { based on } 7 T\end{array}$ & Surgery & Histology \\
\hline \multirow[t]{8}{*}{$\begin{array}{l}38 \text { Considered } \\
\text { non-lesional }\end{array}$} & \multirow[t]{3}{*}{8 Lesional } & $\begin{array}{l}5 \text { Resective surgery (with } \\
\text { ECOG) }\end{array}$ & $\begin{array}{l}2 \text { FCD ILAE type lla } \\
1 \text { FCD ILAE type Ilb } \\
1 \text { mMCD type } 2 \\
1 \text { Proliferative oligodendroglial } \\
\text { hyperplasia with mild malformation } \\
\text { cortical development }\end{array}$ \\
\hline & & $\begin{array}{l}1 \text { Resective surgery with } \\
\text { ACOG planned } \\
1 \text { ECOG planned }\end{array}$ & 2 Yet unknown (FCD suspected) \\
\hline & & 1 Still under evaluation & Yet unknown (FCD suspected) \\
\hline & \multirow[t]{5}{*}{30 Non-lesional } & $\begin{array}{l}6 \text { Resective surgery } \\
\text { (ECOG guided) }\end{array}$ & $\begin{array}{l}2 \text { mMCD type } 2 \\
1 \text { FCD ILAE type lla } \\
1 \text { Normal histology } \\
1 \text { Normal histology (sample error } \\
\text { suspected) } \\
1 \text { Normal histology (suboptimal } \\
\text { assessment due to tissue } \\
\text { fragmentation) }\end{array}$ \\
\hline & & 6 ECOG or S-EEG planned & - \\
\hline & & $\begin{array}{l}12 \text { Rejected for resective } \\
\text { surgery } \\
\text { (1 MST, } 1 \text { VNS) }\end{array}$ & - \\
\hline & & $\begin{array}{l}3 \text { No surgery (declined / } \\
\text { clinical improvement) }\end{array}$ & - \\
\hline & & 3 Still under evaluation & - \\
\hline \multirow{2}{*}{$\begin{array}{l}2 \text { Suspicion of } \\
\text { dual pathology }\end{array}$} & Additional lesion & Resective surgery & mMCD type 2 , no MTS \\
\hline & No additional lesions & Resective surgery & MTS + FCD ILAE type IIla \\
\hline
\end{tabular}

$E S M=$ epilepsy surgery meeting; $E C O G$ = electrocorticography; S-EEG: Stereo EEG; FCD = focal cortical dysplasia; $\mathrm{mMCD}=$ mild Malformation of Cortical Development; MTS = Mesiotemporal (hippocampal) sclerosis. MST = multiple subpial transections. VNS = vagal nerve stimulator.

gyration pattern in combination with enhanced venous vasculature on the $T_{2}{ }^{*}$ sequence, indicating a possible developmental abnormality in the anterior temporal lobe. Selective left anterior temporal lobectomy with hippocampectomy was performed and the hippocampus was found to have normal histology while in the temporal lobe mild architectural abnormalities compatible with mMCD type 2 were confirmed.

After identification of new lesions at 7 tesla we reviewed lower field strength images once more. Even though these were inconspicuous at first, in hindsight, with knowledge of the 7T MRI findings, lesions could be recognized in 3T images in three of nine patients (33\%). 


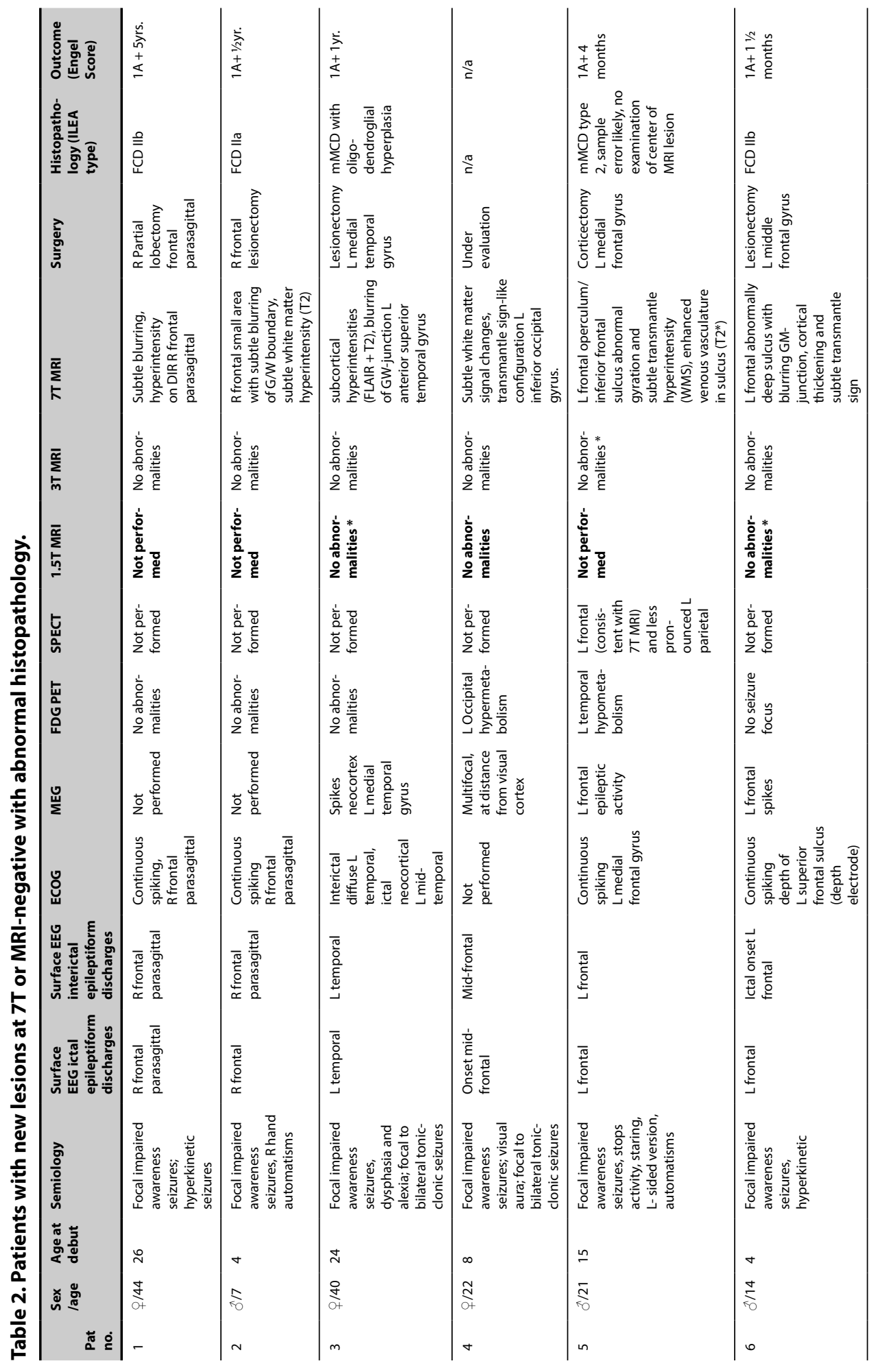




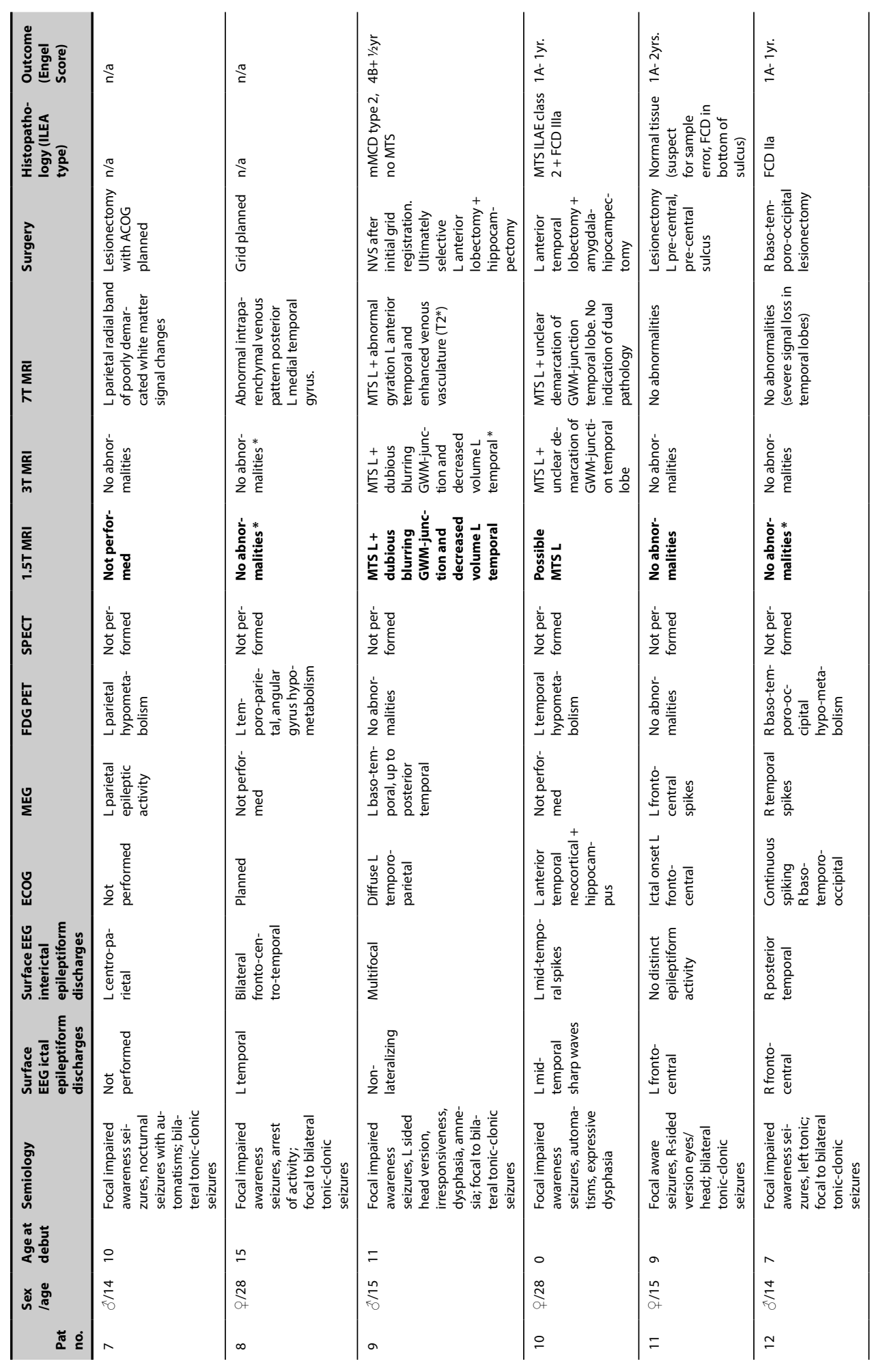




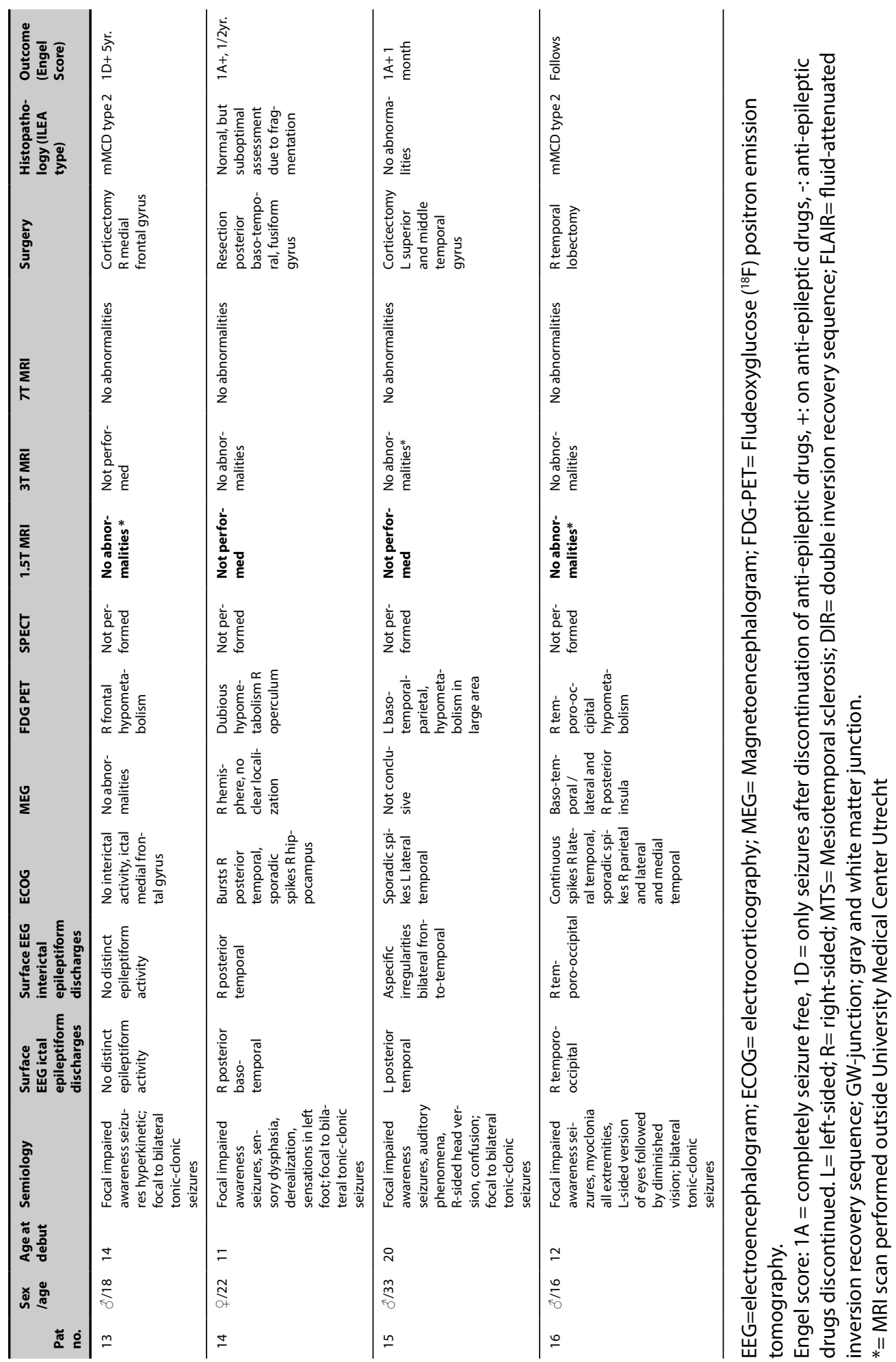


Six patients were operated even though pre-surgical assessment of 7T MRI did not reveal any relevant lesions. They underwent grid-ECOG-guided corticectomy and in three patients histopathological analysis revealed a dysplastic lesion ( 2 mMCD type 2, patient 13 and 16; 1 FCD Ila, patient 12, Table 2). With knowledge of the histological diagnosis, even by hind-sight no lesion could be seen at 7T, however temporal signal loss typical to ultra-high field acquisitions with insufficient correction methods, made assessment of the region of resection impossible in one of these patients (patient 12, Table 2). In another patient (patient 11, Table 2) normal histology was seen, even though highly localized continuous spiking patterns in ECOG registration were strongly suggestive of a small bottom of the sulcus FCD. The difficult access to this lesion precluded en-bloc resection and the deeply located lesion was removed with a surgical aspirator, most likely leading to sample error in the pathological diagnosis. Indeed, retrospectively subtle features indicative of FCD were identified on the 7T MRI.

\section{Discussion}

Of 40 patients with a clinical pre-surgical indication for 7T MRI because of refractory focal epilepsy and presumed normal or nonexplanatory lower-field MRI, in nine (23\%) 7T MRI revealed a lesion, guiding further surgical decision making. Six of these nine patients have been operated upon, with confirmation of FCD ILAE type Ila ( $n=2)$, FCD IIb $(n=1)$, mMCD type II $(n=2)$ or $m M C D$ with oligodendroglial hyperplasia $(n=1)$. One patient is planned for surgical treatment under suspicion of $F C D$, and in one patient with suspected $F C D$ surgery was postponed because of prolonged seizure freedom. One patient is still being evaluated.

A recent study by de Ciantis ${ }^{99}$ evaluated the diagnostic yield of 7T MRI in 21 patients with intractable focal epilepsy and unrevealing 1.5 or 3T MRI. Structural lesions were identified in $6(29 \%)$ of 21 patients, four of whom underwent epilepsy surgery and histopathology confirmed FCD (ILAE type lla $n=2 ;\|l b n=1 ;\| l l a n=1$ ). In that study, however, only half of the six presumed MR-negative patients in whom $7 T$ revealed a new lesion underwent previous MRI at 3T, whereas in our series all nine patients with new lesions underwent 3T MRI before. Our findings therefore truly reflect the added value of 7T over the current standard, 3T MRI, in the pre-surgical evaluation.

\section{Visualization of FCD}

Several MRI features characterize $F C D^{102}$, but with great variety in conspicuity. Especially in $\mathrm{mMCD}$ and FCD type $\mathrm{l}$, changes can be very subtle ${ }^{42}$ and indistinguishable from signal averaging and partial volume effects. The same phenomena can hinder appreciation of blurring of the gray-white matter junction, a tell-tale feature of FCD. Sub-millimeter resolution and slice thickness greatly reduce the detrimental signal averaging and partial 
volume effects. Moreover, multi-axial images are needed to be able to optimally assess the highly convoluted cerebral cortex. Subtle signal changes can be obvious in one plane but completely obscured by the cortical anatomy in another plane. In some cases even, the sole identifier can be an abnormal gyration pattern, only recognizable in high quality 3D image sets $42,102,105$.

With current coil and processing techniques it is not possible to achieve sub-millimeter high resolutions in all essential sequences $\left(T_{1}, T_{2} \text {, FLAIR, } T_{2}^{*}\right)^{100,106}$ at $3 T$, while maintaining tissue contrast and staying within acceptable acquisition times. At 7T this is currently possible, and is its most important strength. On the other hand advances in coil design and data processing could possibly also achieve this at lower field strengths in the future ${ }^{107}$.

\section{Inability of $\mathbf{7}$ tesla MRI to visualize dysplasia}

In 30 out of 38 (79\%) patients with non-lesional lower-field MRI, 7T MRI did not show epilepsy-related lesions either. Possibly there is no structural substrate, but it is known that a portion of these patients do prove to have histological abnormalities eventually ${ }^{42}$. In our series three patients had confirmed FCD or mild malformation of cortical development in the absence of any reported MR abnormalities at 7T, even after retrospective review. In one patient even though histology was normal, probably due to sample error, based on ECOG findings FCD is likely. In retrospect there are signs of a bottom of sulcus FCD on the 7T MRI.

There are several possible reasons why assessment of the high resolution images does not always provide the pivotal information hoped for.

Firstly, experience in 7T MRI assessment is still limited compared to 1.5 and 3T MRI, the clinical standard for many years. The added detail is sometimes a double-edged sword; partial volume effects are greatly reduced and much smaller structures are visible. But as a result the images reveal a great number of structures for which it can be problematic to differentiate between physiological clinically meaningless variation and true pathology. Secondly, thorough evaluation of 7T examinations is relatively time consuming. With constructed slice thickness of $0.5 \mathrm{~mm}$, a completed scan protocol easily produces up to 4000 slices. Possibly lesions are missed if no extra time is reserved for the clinical evaluation. The number of slices and thus evaluation time could be reduced by increasing slice thickness and reducing reconstruction planes, but this would likely negate the advantage of performing 7T MRI.

Thirdly, with the increase in field strength come more artefacts ${ }^{97}$. Magnetic susceptibility effects and field inhomogeneities cause artefacts and signal loss. Temporal lobes are particularly problematic with varying, but occasionally severe signal loss making assessment impossible. Several techniques to counter these artefacts are already successfully used and more are under development. A rather simple but effective tool we routinely use since 2015 are the dielectric calcium titanate pads with passive RF shimming qualities which 
have shown to greatly reduce signal loss in the temporal lobes ${ }^{101}$. With growing clinical and technical experience, one would expect the diagnostic value of 7T MRI to further increase.

\section{Strengths and weaknesses}

We chose to evaluate 7T MRI in daily clinical practice, with the conclusion of the multidisciplinary meetings as final assessment. This design reflects how the added information from 7T MRI affects decision making and how its use may benefit patient care. Naturally this setting does not allow for a formal and perfectly objective comparison of the diagnostic accuracy between 7T and lower field strength, which would require systematic scoring by multiple observers in a controlled environment. For the decision for surgical candidacy, clinical information and ancillary tests are combined for more robust assessments. We are convinced that lesion detection on MRI is more sensitive with fewer false-positives when taking supporting information and other tests in account. Omitting this aspect in evaluating the value of 7T can lead to results that are not fully applicable to clinical practice. However, 7T MRI scans are offered later in the diagnostic work-up, and consequently in 17 of 40 (43\%) additional ancillary test were available during the 7T review. This was true for five of nine (55\%) patients with new 7T MRI findings. The added information available during multidisciplinary assessment of the 7T scans may have contributed to a better detection rate. Moreover, acquisition time is $45-50$ minutes at 7T, compared to 25 minutes for standard protocols at 1.5 or $3 \mathrm{~T}$ in our center. Hence, the longer acquisition is an additional benefit (leading to either an increase in signal-to-noise, or higher resolution) on top of the increase in field strength.

\section{Conclusion}

Our study demonstrates that the use of ultra-high field MRI with an extensive scanning protocol can improve lesion detection, compared to the current clinical standards. It seems worthwhile to further invest in improving MRI techniques for patients with focal refractory epilepsy. In our series of 40 patients with refractory focal epilepsy, multidisciplinary evaluation of 7T MRI identified clinically relevant additional malformation of cortical development lesions in nine patients (23\%). 7T MRI delivers on the promise to improve detection of focal cortical dysplasia and mild malformation of cortical development in patients with intractable epilepsy. 
Supplementary table 1. Scan Parameters.

\begin{tabular}{|c|c|c|c|c|c|c|c|c|}
\hline & & TE ms & TI ms & TR ms & $\begin{array}{l}\text { FA } \\
\text { degrees }\end{array}$ & $\begin{array}{l}\text { Acquired } \\
\text { resolution } \\
\mathrm{mm}\end{array}$ & $\begin{array}{l}\text { Matrix (AP x } \\
\text { RL) x slices }\end{array}$ & $\begin{array}{l}\text { Acquisition } \\
\text { time } \\
\text { min:sec }\end{array}$ \\
\hline \multirow[t]{5}{*}{ 7T } & 3D $T, w$ TFE & 2.9 & 1200 & 9 & 6 & $0.81 / 0.81 / 0.80$ & $248 \times 312 \times 475$ & $9: 34$ \\
\hline & 3D $T_{2} w$ TSE & 302 & - & 3200 & 90 & $0.70 / 0.70 / 0.70$ & $356 \times 357 \times 543$ & $10: 24$ \\
\hline & 3D T2*w & 27 & - & 88 & 24 & $0.50 / 0.50 / 0.50$ & $480 \times 381 \times 600$ & $7: 23$ \\
\hline & 3D MP-FLAIR & 300 & 2200 & 8000 & 90 & $0.80 / 0.82 / 1.00$ & $312 \times 304 \times 380$ & $8: 40$ \\
\hline & 3D WMS & 2.0 & 600 & 6.73 & 4.5 & $0.80 / 0.80 / 0.80$ & $320 \times 320 \times 474$ & $8: 42$ \\
\hline \multirow[t]{6}{*}{ 3T } & $\mathrm{S} T_{1} \mathrm{SE}$ & 15 & - & 870 & 70 & $0, .90 / 1.12 / 3.00$ & $256 \times 205 \times 31$ & $3: 00$ \\
\hline & $\begin{array}{l}t \text { dual TSE } \\
\text { SPAIR }\end{array}$ & $10 / 80$ & - & 2881 & 90 & $0.65 / 0.87 / 4.00$ & $352 \times 210 \times 26$ & 4:07 \\
\hline & t DW & 68 & - & 3298 & 90 & $0.96 / 1.21 / 4.00$ & $240 \times 190 \times 26$ & 1:09 \\
\hline & c FLAIR & 120 & 2800 & 11000 & 90 & $0.65 / 0.84 / 4.00$ & $336 \times 210 \times 40$ & $6: 03$ \\
\hline & $3 \mathrm{D} T_{1}$ FFE & 3.3 & & 6.6 & 8 & $0.60 / 1.10 / 1.20$ & $400 \times 200 \times 300$ & $5: 31$ \\
\hline & $\begin{array}{l}\text { 3D } T_{1} \text { FFE } \\
\text { gado }\end{array}$ & 3.3 & & 6.6 & 8 & $0.60 / 1.10 / 1.20$ & $400 \times 200 \times 300$ & $5: 31$ \\
\hline \multirow[t]{6}{*}{$1.5 \mathrm{~T}$} & s $T_{1}$ SE & 12 & - & 604 & 90 & $0.98 / 1.62 / 4.00$ & $256 \times 154 \times 27$ & $1: 34$ \\
\hline & t dual SE & $30 / 100$ & - & 2369 & 90 & $0.90 / 1.28 / 6.00$ & $256 \times 171 \times 19$ & $6: 47$ \\
\hline & t DW SSH & 81 & - & 2576 & 90 & $1.80 / 2.99 / 6.00$ & $128 \times 77 \times 19$ & $0: 15$ \\
\hline & c $T_{2}$ FLAIR TSE & 140 & 2600 & 11000 & 90 & $0.90 / 1.27 / 4.00$ & $256 \times 144 \times 40$ & $5: 08$ \\
\hline & $\mathrm{S} T_{1}$ FFE & 4.6 & - & 25 & 30 & $1.00 / 1.00 / 1.00$ & $240 \times 240 \times 175$ & $4: 52$ \\
\hline & $s T_{1}$ FFE gado & 4.6 & - & 25 & 30 & $1.00 / 1.00 / 1.00$ & $240 \times 240 \times 175$ & $4: 52$ \\
\hline
\end{tabular}

$\mathrm{t}=$ transverse; $\mathrm{c}=$ coronal; $\mathrm{s}=$ sagittal; TFE $=$ Turbo field echo (gradient echo); TSE $=$ Turbo Spin Echo; $T_{2}^{*} \mathrm{~W}=T_{2}^{*}$ weighted (susceptibility contrast weighted); FFE = Fast Field Echo (gradient echo); MP-FLAIR = magnetization prepared - FLAIR $^{108}$; WMS = White Matter Suppression sequence (gradient echo); SE = Spin Echo; SPAIR = Spectral selection Attenuated Inversion Recovery (fat suppression) DW = diffusion weighted; gado = gadolinium intravenous contrast; dual = dual echo; $\mathrm{SSH}=$ single-shot 


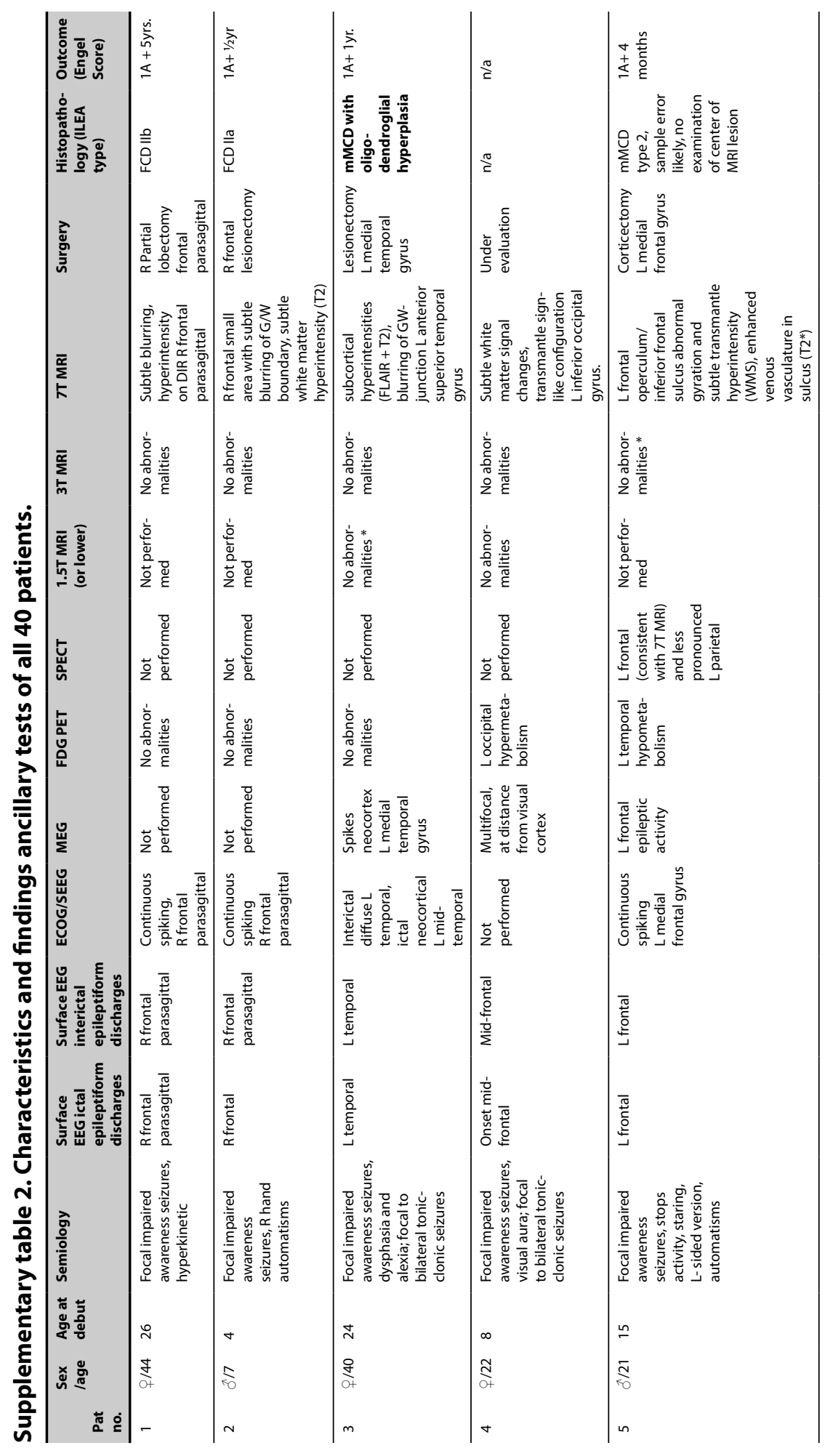




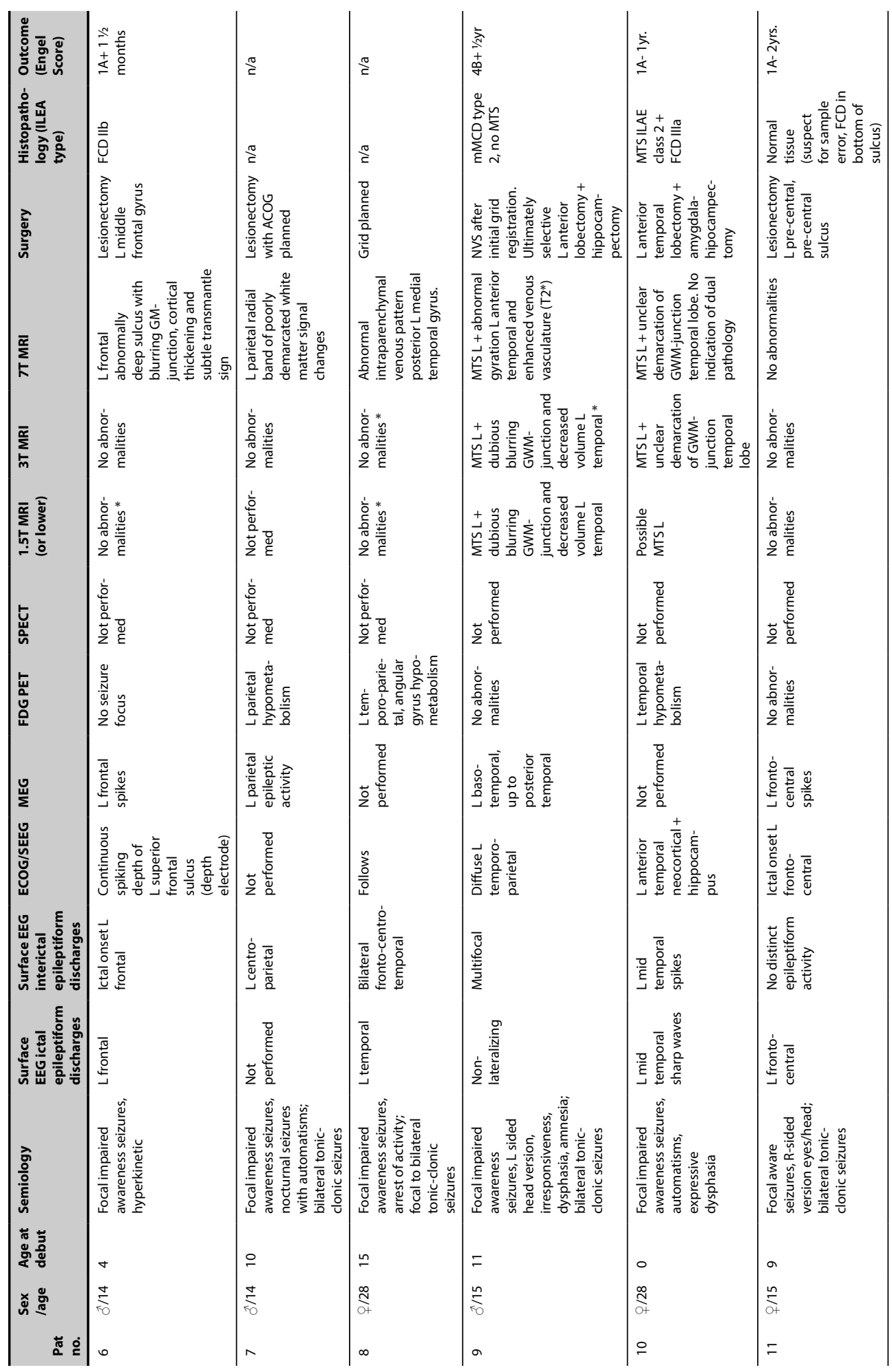




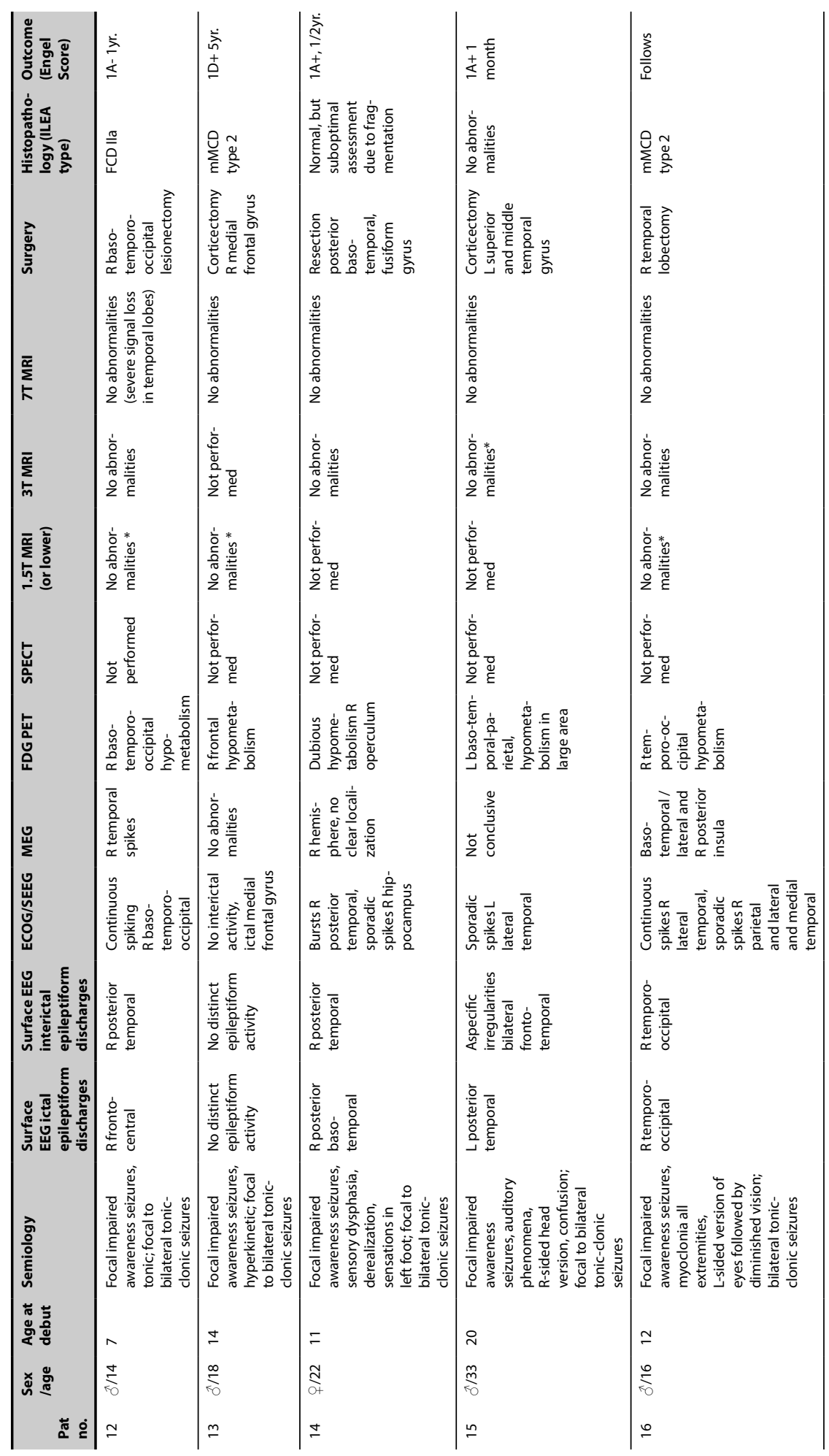


66 | Chapter 4

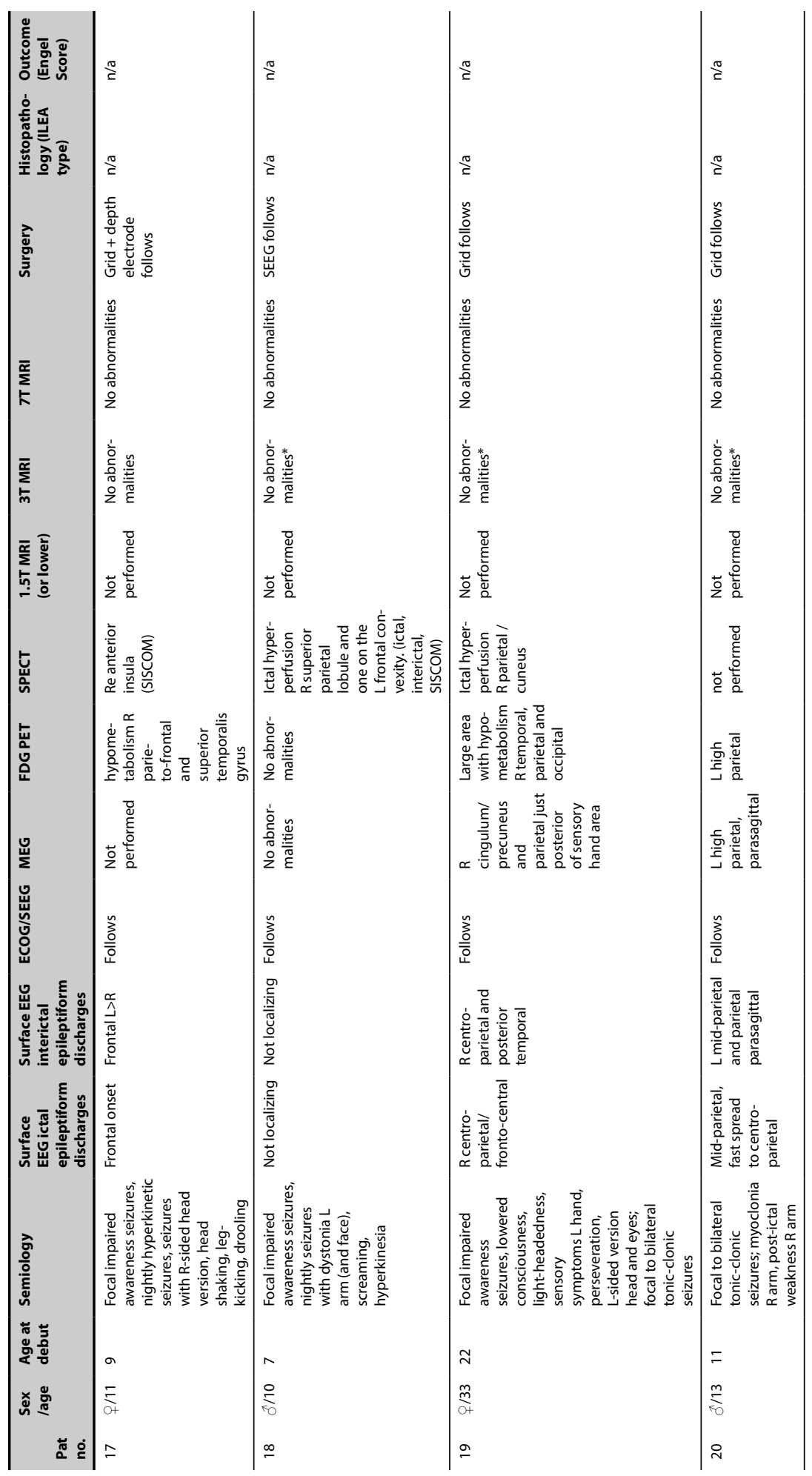




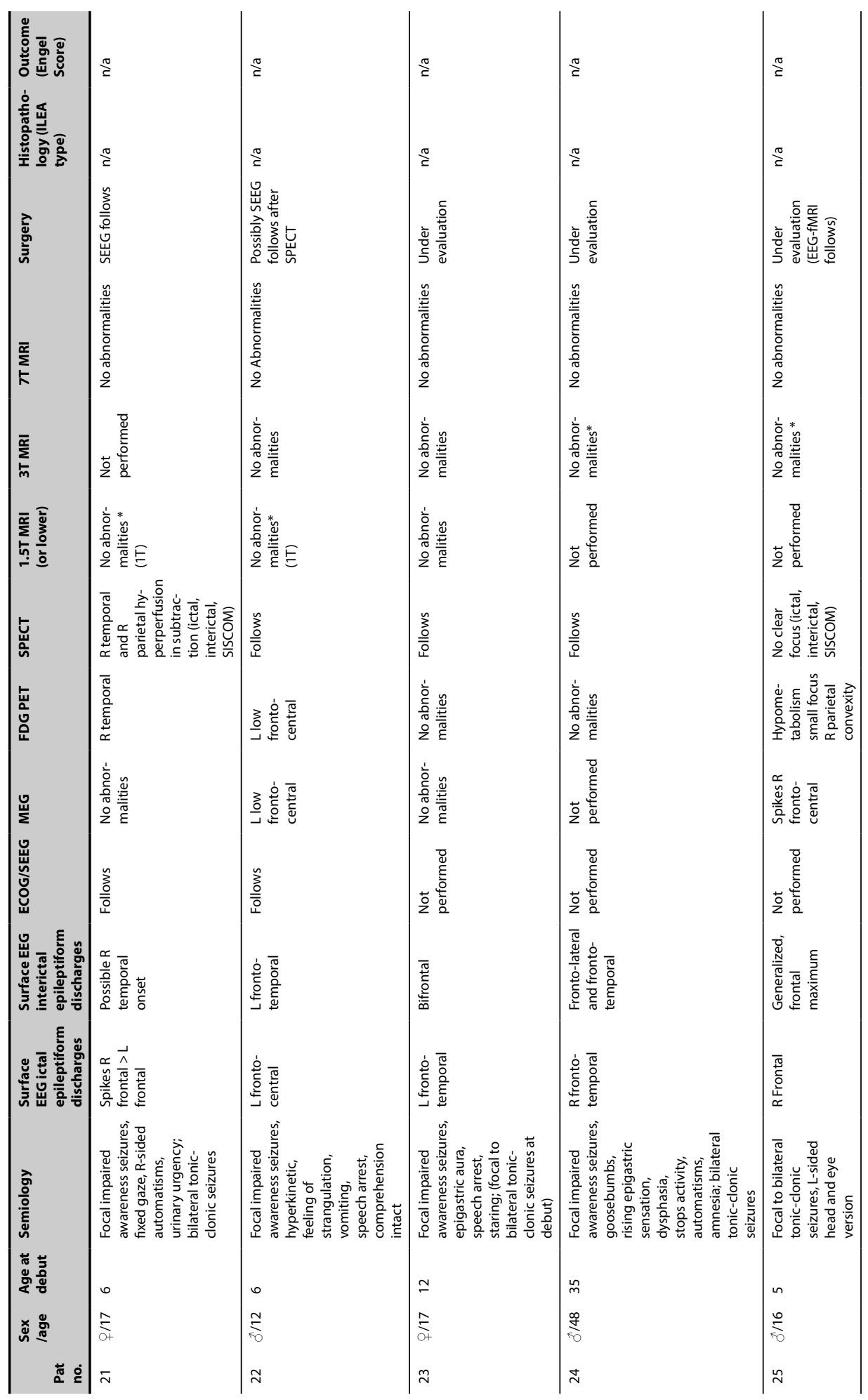


68| Chapter 4

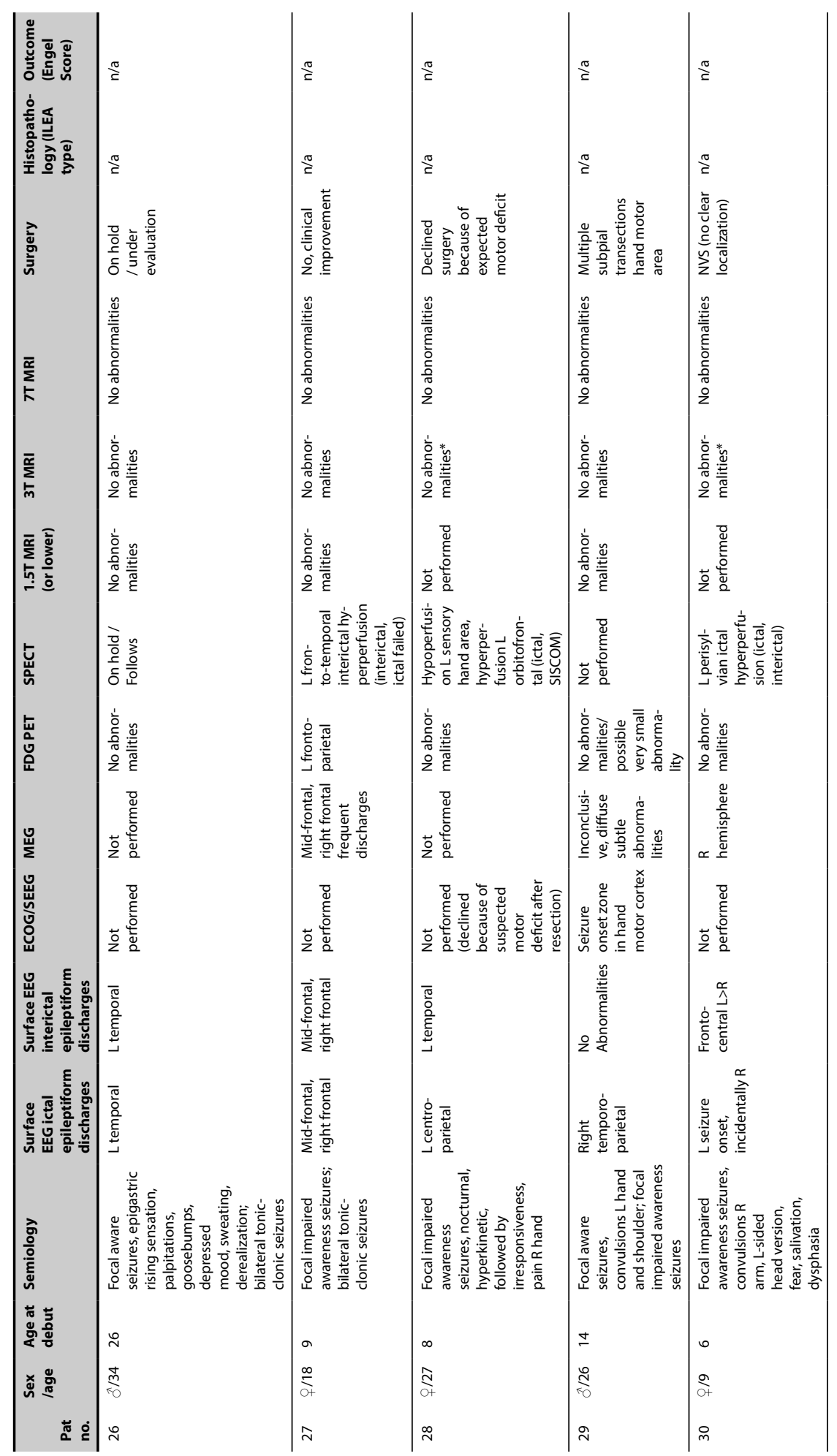




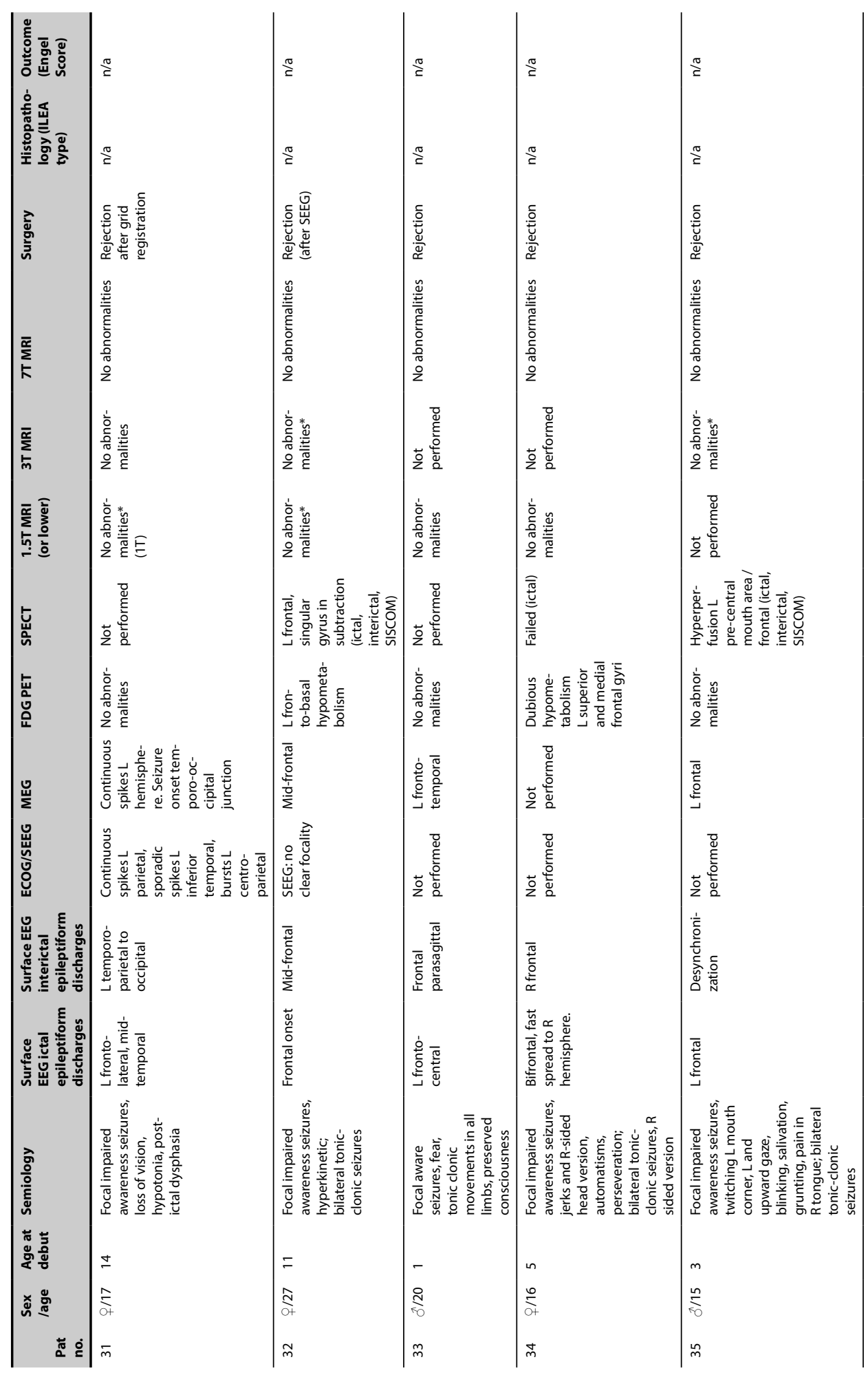




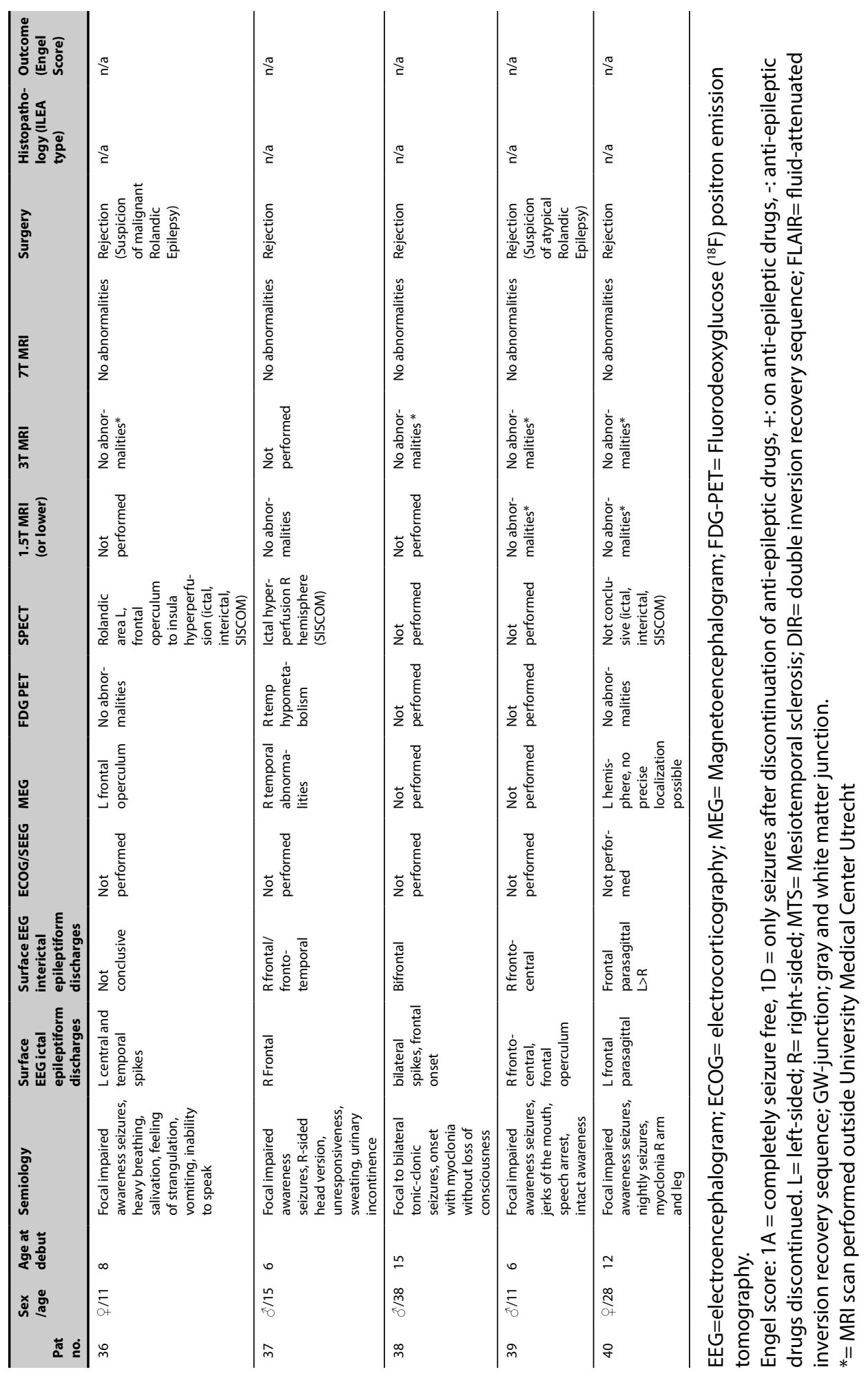


7T MRI improves detection of FCD in patients with refractory focal epilepsy | 71 



\section{TESLA T2 ${ }_{2}^{*}$-WEIGHTED MRI AS A TOOL TO IMPROVE DETECTION OF FOCAL CORTICAL DYSPLASIA - A CASE SERIES}

Tim J. Veersema, Pieter van Eijsden, Peter H. Gosselaar Jeroen Hendrikse, Jaco J.M. Zwanenburg, Wim G.M. Spliet, Eleonora Aronica Kees P.J. Braun, Cyrille.H. Ferrier 
Focal cortical dysplasia is one of the most common underlying pathologies in patients who undergo surgery for refractory epilepsy. Absence of a MRI-visible lesion necessitates additional diagnostic tests and is a predictor of poor surgical outcome.

We describe a series of six patients with refractory epilepsy due to histopathologically proven focal cortical dysplasia, of whom pre-surgical 7 tesla $T_{2}{ }^{*}$-weighted MRI was acquired. In four out of six patients $T_{2}{ }^{*}$ sequences showed areas of marked superficial hypointensity co-localizing with the epileptogenic lesion. 7 tesla $T_{2}{ }^{*}$ hypointensities overlying focal cortical dysplasia may represent leptomeningeal venous vascular abnormalities associated with the underlying dysplastic cortex. Adding $T_{2}^{*}$ sequences to the MRI protocol may aid in the detection of focal cortical dysplasias. 


\section{Introduction}

Focal cortical dysplasia (FCD) is one of the most common underlying pathologies in patients who undergo surgery for refractory epilepsy. A substantial percentage of these lesions is not visible with a routine 1.5 tesla (T) or $3 \mathrm{~T} \mathrm{MRI;} \mathrm{37 \%} \mathrm{for} \mathrm{FCD} \mathrm{type} \mathrm{I} \mathrm{and} \mathrm{15 \%} \mathrm{for} \mathrm{type} \mathrm{FCD}$ type II in a review of surgical series from 2000 to $2008 ; 36 \%$ and $2 \%$ in a $2000-2007$ UCLA series of 97 patients ${ }^{70}$. No visible lesion on MRI is a predictor of poor surgical outcome and necessitates additional diagnostic studies which may include invasive intracranial electrode registrations ${ }^{91}$. Advances in imaging techniques are expected to improve the detection of epileptogenic lesions.

Susceptibility-based contrast MRI sequences are sensitive to the susceptibility effect created by the paramagnetic property of deoxyhemoglobin in blood, and thus augment the visibility of the cerebral venous microvasculature ${ }^{109}$. Sequences as $T_{2}{ }^{*}$ and Susceptibility-Weighted Imaging (SWI) have been proven useful in the detection and characterization of a variety of cerebral vascular abnormalities (e.g. Sturge-Weber Syndrome, cavernous malformations), as well as hemorrhagic, calcified or iron-containing lesions ${ }^{110}$. Reports on susceptibilitybased contrast sequences in epilepsy patients are limited and mostly describe the benefits for detection and characterization of calcified lesions, not for the detection of otherwise inconspicuous lesions, such as FCD ${ }^{111}$. In this series we illustrate the potential of the $T_{2}{ }^{*} \mathrm{MRI}$ sequence at $7 \mathrm{~T}$ in assisting the detection of FCD.

\section{Methods}

We retrospectively selected six consecutive patients from the Dutch Epilepsy Surgery Program with refractory epilepsy due histopathologically confirmed FCD, of whom $T_{2}^{*}$ images at 7T were acquired pre-surgically between November 2008 and November 2014. These patients either had lesions suggestive of FCD on 3T $(n=2)$ or had normal 3T MRIs, with semiology, electroencephalogram and telemetry findings strongly suggesting a structural focal abnormality $(n=4)$. 3T MRI was deemed normal if no indications for structural abnormalities were identified on assessment by expert neuroradiologists and during review by the Dutch Epilepsy Surgery Program board - that includes neurosurgeons, neurologists, and neuroradiologists with expertise in the field of epilepsy. 3T MRI-negative patients underwent additional pre-surgical evaluation, including 7T MRI and PET, SPECT, or MEG in order to localize the epileptogenic zone. In addition 7T MRI was used to further characterize the lesions for the two subjects with lesions on 3T.

The 7T MRI protocol included (conventional) 3D FLAIR, 3D Double Inversion Recovery, 3D $T_{1}$ and 3D $T_{2-}$ weighted sequences and a previously described $T_{2}{ }^{*}$-weighted sequence ${ }^{112}$. Parameters used for the gradient echo $T_{2}{ }^{*} \mathrm{MRI}$ were: isotropic $0.5 \mathrm{~mm}$ resolution, echo time $27 \mathrm{~ms}$, flip angle $24^{\circ}$, repetition time $57-93 \mathrm{~ms}$ (shortest possible), with EPI and flow 
compensation. For two patients scans were acquired with $0.6 \mathrm{~mm}$ resolution (isotropic), echo time $20 \mathrm{~ms}$, flip angle $20^{\circ}$ and repetition time $25-26 \mathrm{~ms}$. Images were acquired on a Phillips 7T system (Philips Healthcare, Cleveland, $\mathrm{OH}$, USA) with a volume transmit and 16or 32-channel receive head coil (Nova Medical, Wilmington, MA, USA).

7T $\mathrm{T}_{2}^{*}$ images were reviewed for abnormalities co-localizing with epileptogenic zones. To aid visual detection of hypointense structures, minimum intensity projections of $T_{2}{ }^{*}$ sequences were constructed with $5 \mathrm{~mm}$ slab thickness. Following resective surgery, tissues were histopathologically classified according to ILAE guidelines ${ }^{13}$.

Informed consent was obtained for use of patient data. The report complies with the declaration of Helsinki.

In addition, we reviewed 7T $T_{2}{ }^{*}$ images of eight control subjects (four male, four female,

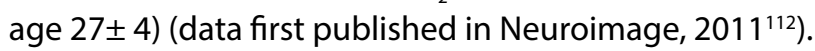

\section{Case Series}

Four of six patients had normal 3T MRI findings; in two of these, conventional 7T MRI sequences $\left(T_{1}, T_{2}\right.$, Double Inversion Recovery, and FLAIR) sequences revealed a lesion suggestive of FCD. In two other patients, abnormalities were seen both on 3T and on 7T images. All six patients underwent resective surgery and had histopathological confirmation of FCD (ILAE type Ib in patient II, type Ila in patient V and VI, type IIb in patient I and IV, and mild Malformation of Cortical Development type 2 in Patient III). In four of these patients (patients I-II Figure 1A, patients III-IV Figure 1B) 7T $T_{2}{ }^{*}$ showed areas containing marked hypointensities with a branched, partly tortuous configuration, and a signal compatible with venous blood, suggestive of increased venous vasculature in the sulci neighboring the malformed cortex.

Because the described $\mathrm{T}_{2}{ }^{*}$ signal changes appeared to be located in the leptomeningeal tissue or subarachnoid space overlying the malformed cortex, we looked for a histopathological correlate in the leptomeninges of the operated patients. Unfortunately, due to the locations of the malformations, respectively in the bottom of a sulcus and interhemispherically, en-bloc resection with intact leptomeningeal structures was only possible in one patient (III). This lesion was classified as mMCD type 2, with fibrotic and thick meninges containing prominent vascular structures. (supplementary figure 1).

In summary, hypointensities on $T_{2}{ }^{*}$ were identified in four out of six consecutive patients with histologically proven FCD of whom 7T $T_{2}{ }^{*}$ images where available. Table 1 lists clinical and imaging characteristics of all six patients. 

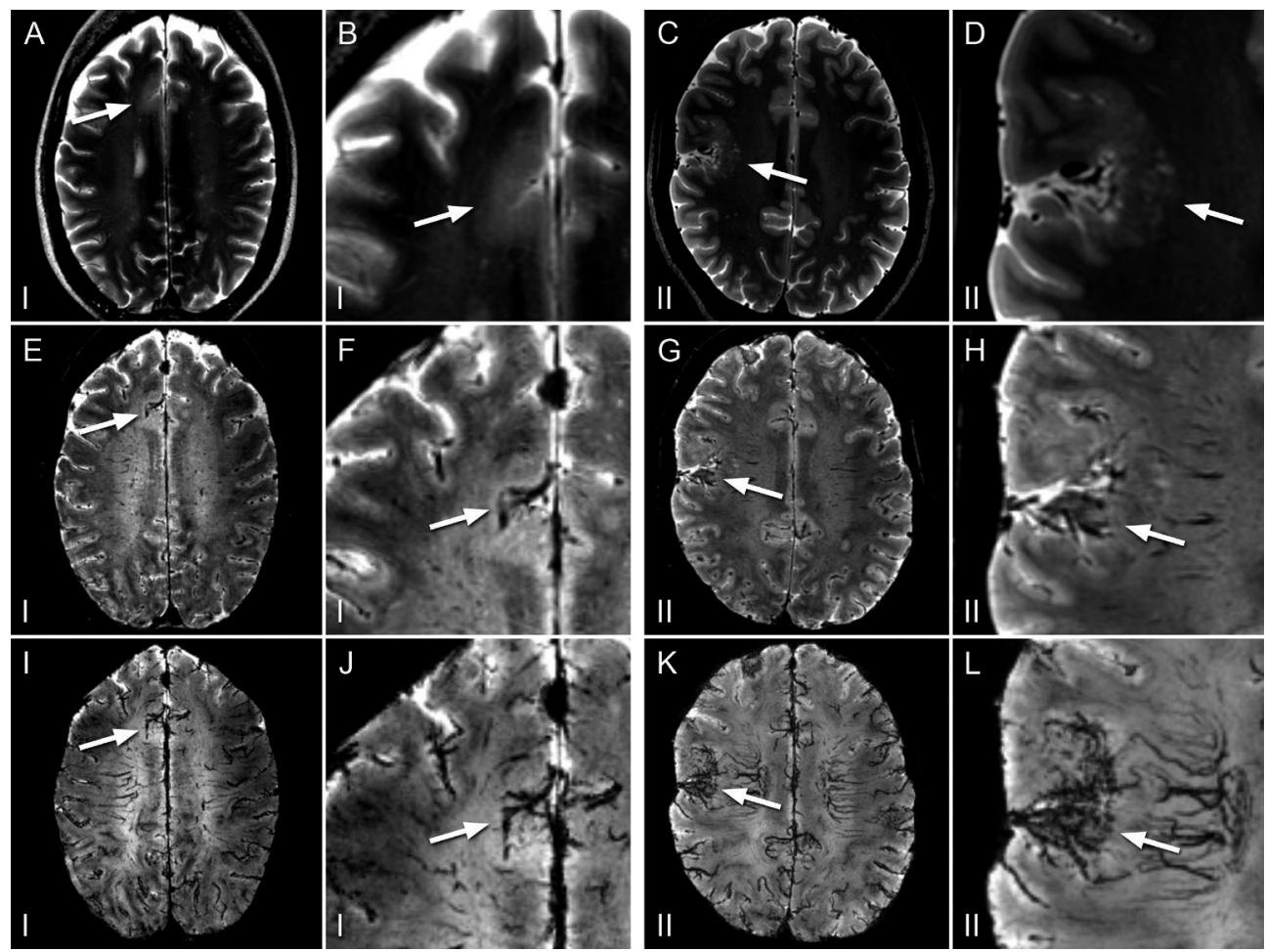

Figure 1A. MRI Findings of patients with proven $F C D$ and $T_{2}{ }^{*}$ signal changes.

7T $T_{2}(\mathrm{~A}-\mathrm{D}), T_{2}{ }^{*}(\mathrm{E}-\mathrm{H})$ and $T_{2}{ }^{*}$ minimum intensity projection (I-L), transverse reconstructions. Lesions depicted in detail in $B, D, F, H, J$ and $L$.

Patient I; FCD ILAE type Ilb (A, B, E, F, I, J). No lesion identified on 3T MRI, subtle gray-white matter junction blurring is seen on 7T $T_{2}$ weighted MRI $(A, B)$. On $T_{2}{ }^{*}(E, F)$ the neighboring sulcus seems to contain prominent vasculature. $T_{2}^{*}$ minimum intensity projection $(\mathrm{I}, \mathrm{J})$ aids in the visual detection. Patient II; FCD ILAE type Ib (C, D, G, H, K, L). $T_{2}$-weighted MRI (C, D) shows grey-white matter junction blurring and cortical thickening indicative of $F C D$. In the same area $T_{2}^{*}(G, H)$ shows a wide sulcus with prominent vascular structures. $T_{2}{ }^{*}$ minimum intensity projection $(\mathrm{K}, \mathrm{L})$ strongly emphasizes the increased vasculature.

On 7T $T_{2}{ }^{*}$ images and corresponding minimum intensity projections of eight healthy volunteers, symmetrical venous leptomeningeal vascular structures are clearly visible but the phenomenon described above was not identified. (See supplementary Figure 2 for $7 \mathrm{~T}$ $T_{2}{ }^{*}$ images of a healthy subject).

\section{Discussion}

We describe the observation of 7T $T_{2}{ }^{*}$ hypointensities overlying pathologically proven $(n=4)$ epileptogenic malformations of cortical development. We propose that these signal abnormalities reveal a pathological change in the leptomeningeal venous vasculature. The 

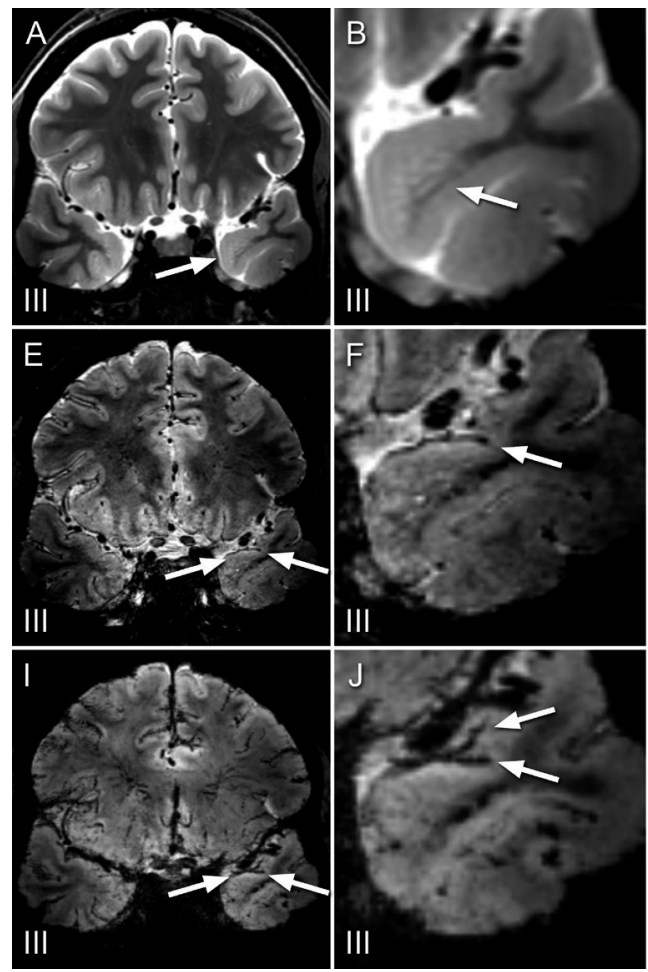
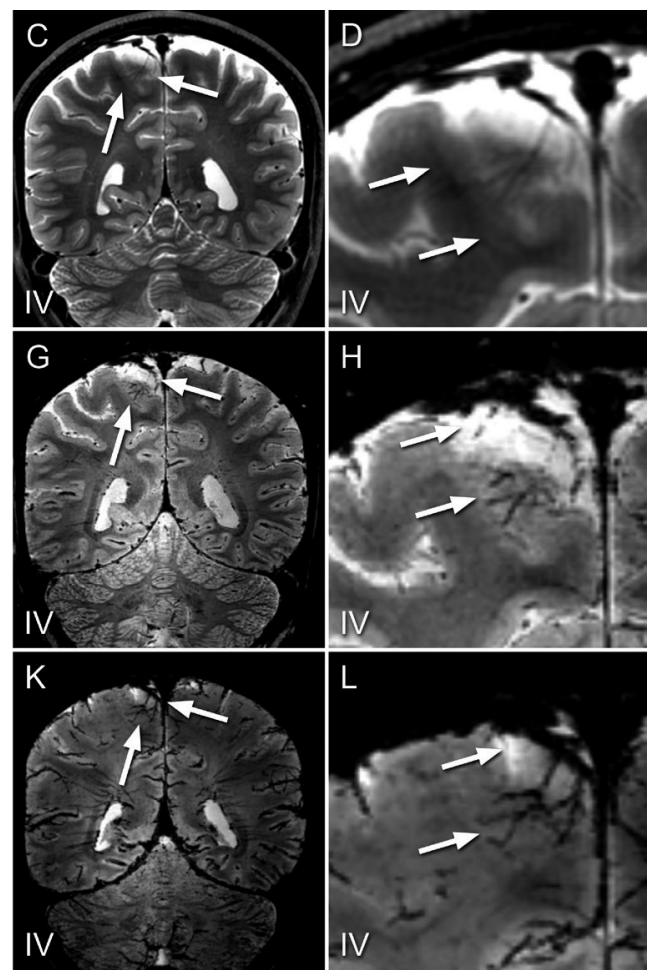

Figure 1B. MRI Findings of patients with proven $F C D$ and $T_{2}^{*}$ signal changes.

7T $T_{2}(\mathrm{~A}-\mathrm{D}), T_{2}{ }^{*}(\mathrm{E}-\mathrm{H})$ and $T_{2}{ }^{*}$ minimum intensity projection (I-L), coronal reconstructions. Lesions depicted in detail in $B, D, F, H, J$ and $L$.

Patient III; mild malformation of cortical development type 2 ( $A, B, E, F, I, J)$. On $T_{2}(A, B)$, blurring and subcortical hyperintensity represent developmental malformation. $T_{2}{ }^{*}(E, F)$ shows a wide Sylvian fissure but no clearly appreciable vascular changes. On $T_{2}{ }^{*}$ minimum intensity projection (I, $J)$ there appears to be an increase in vascular signal in the superior temporal pole.

Patient IV (C, D, G, H, K, L); FCD ILAE type Ilb. On $T_{2}$ (C, D) notable large extracerebral space central parasagittal containing a large vein, but without evident dysplastic characteristics but. $T_{2}{ }^{*}(G, H)$ shows the large vein and smaller vasculature that drains from the dysplastic cortex (as proven by histology). Enhanced conspicuity on $T_{2}{ }^{*}$ minimum intensity projection $(\mathrm{K}, \mathrm{L})$.

location in sulci and on the cortical surface, the configuration, and a $T_{2}{ }^{*}$ signal compatible with venous blood all support this hypothesis. The origin of the observed $T_{2}{ }^{*}$ hypointensities could be either, or a combination, of the following:

1. Increased blood volume may be the result of increased vascular diameter or density in the context of a developmental anomaly of the leptomeningeal vasculature, parallel to the developmental malformation of the underlying cortex. The leptomeningeal vascular network is formed from a gestational age of 8 weeks, starting as the pial capillary anastomotic plexus which produces penetrating vessels into the cortex ${ }^{113}$. It is conceivable that in addition to immaturity and abnormal migration of neurons, the vascular network may show analogous 


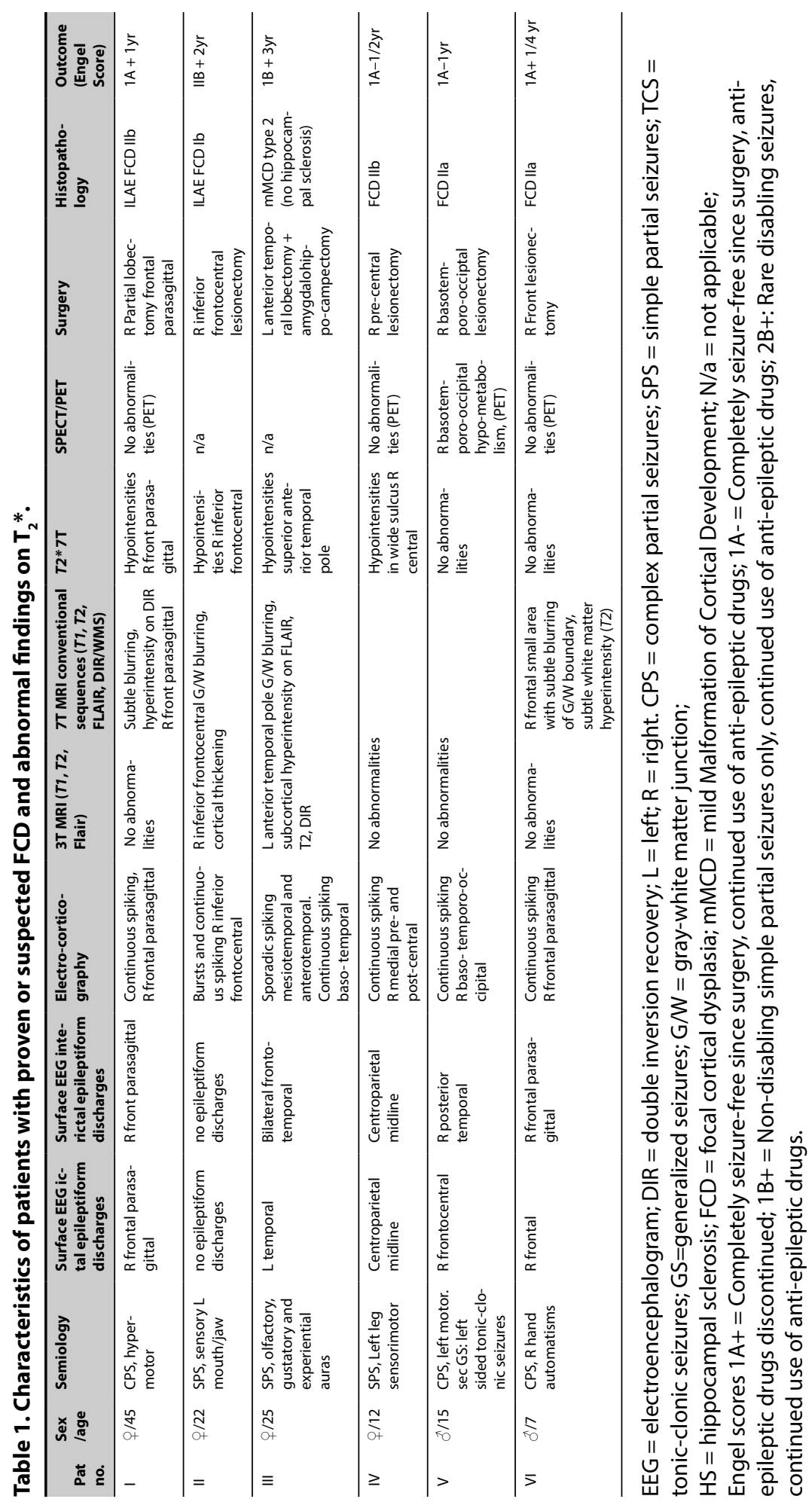


abnormalities in dysplastic cortex, resulting in abnormal configuration of superficial cortical vasculature.

2. Paroxysmal increased metabolic demand associated with epileptic activity ${ }^{114}$ may lead to reactive vascular changes in the form of hypervascularization. An increase in venous vascular diameter or density results in a greater deoxygenated blood volume per voxel and concomitant $T_{2}{ }^{*}$ changes.

3. A focal increase in deoxyhemoglobin may be explained by altered hemodynamics. Other studies showed that interictal hypometabolism was associated with decreased perfusion on arterial spin labelling MRI ${ }^{115}$, while cortical activation is generally linked to positive blood oxygen dependent signal changes caused by an increase in oxygenated blood ${ }^{116}$. Decreased perfusion in epileptogenic lesions may lead to an interictal increase in venous deoxyhemoglobin, visible as $T_{2}{ }^{*}$ hypointensity.

The clinical relevance of the described phenomenon is that it might be indicative of underlying epileptogenic cortex and even distinctive in cases where conventional MRI sequences reveal no abnormalities.

Two additional patients with refractory epilepsy evaluated in our center had clinical and electrophysiological characteristics indicative of a structural seizure focus but no supportive findings on 3 and 7T MRI. Retrospective review of 7T MRI $T_{2}^{*}$ images showed hypointensities that might have provided clues for localizing the seizure focus (data not shown). Lack of concordance between other ancillary diagnostic results prevented surgical treatment.

Comparison of $T_{2}{ }^{*}$-weighted sequences acquired at 7T and at lower field strength was not possible since this sequence is not routinely performed on 1.5 or 3T MRI in epilepsy patients in our center. Fine vascular structures should be better detectable on 7T systems because of the higher spatial resolution and the positive relation between field strength and $T_{2}{ }^{*}$ effects ${ }^{109}$. Because of the nature of the pathology and the still experimental status of 7T $\mathrm{MRI}$ it is difficult to obtain $T_{2}{ }^{*}$ images in large groups of patients for assessing the presence of the described phenomenon.

An important limitation is that we report a qualitative analysis of the $T_{2}{ }^{*}$ signal change. Furthermore histopathological validation of changes in leptomeningeal tissues is limited, because leptomeninges could only be assessed in one surgical specimen. While the impression of increased vasculature on $T_{2}^{*}$ images is congruent with the leptomeningeal histological findings (thick and fibrosed meninges containing large caliber vessels) this may be a non-specific finding in patients with epilepsy. To elucidate the histopathological substrate for this potential marker of subtle epileptogenic lesions and assess its clinical 
relevance, studies on larger groups of patients are needed, including histopathological analysis of leptomeningeal vascular structures.

Despite its limitations, this case series suggests that it may be worthwhile further exploring the possibilities of using $T_{2}{ }^{*}$ sequences at 7T - and possibly also at lower field strength - to improve the detection of FCD in patients with refractory epilepsy.

\section{Conclusion}

We report a novel, 7T MR $T_{2}{ }^{*}$ finding co-localizing with FCD. This hypointense signal may indicate an increase in vascular prominence in the leptomeningeal vascular network overlying the dysplastic cortex. Adding $T_{2}{ }^{*}$-weighted sequences to the 7T MRI protocol may aid in the detection of FCDs and guiding effective epilepsy surgery, while obviating the need for additional (invasive) diagnostic tests. 

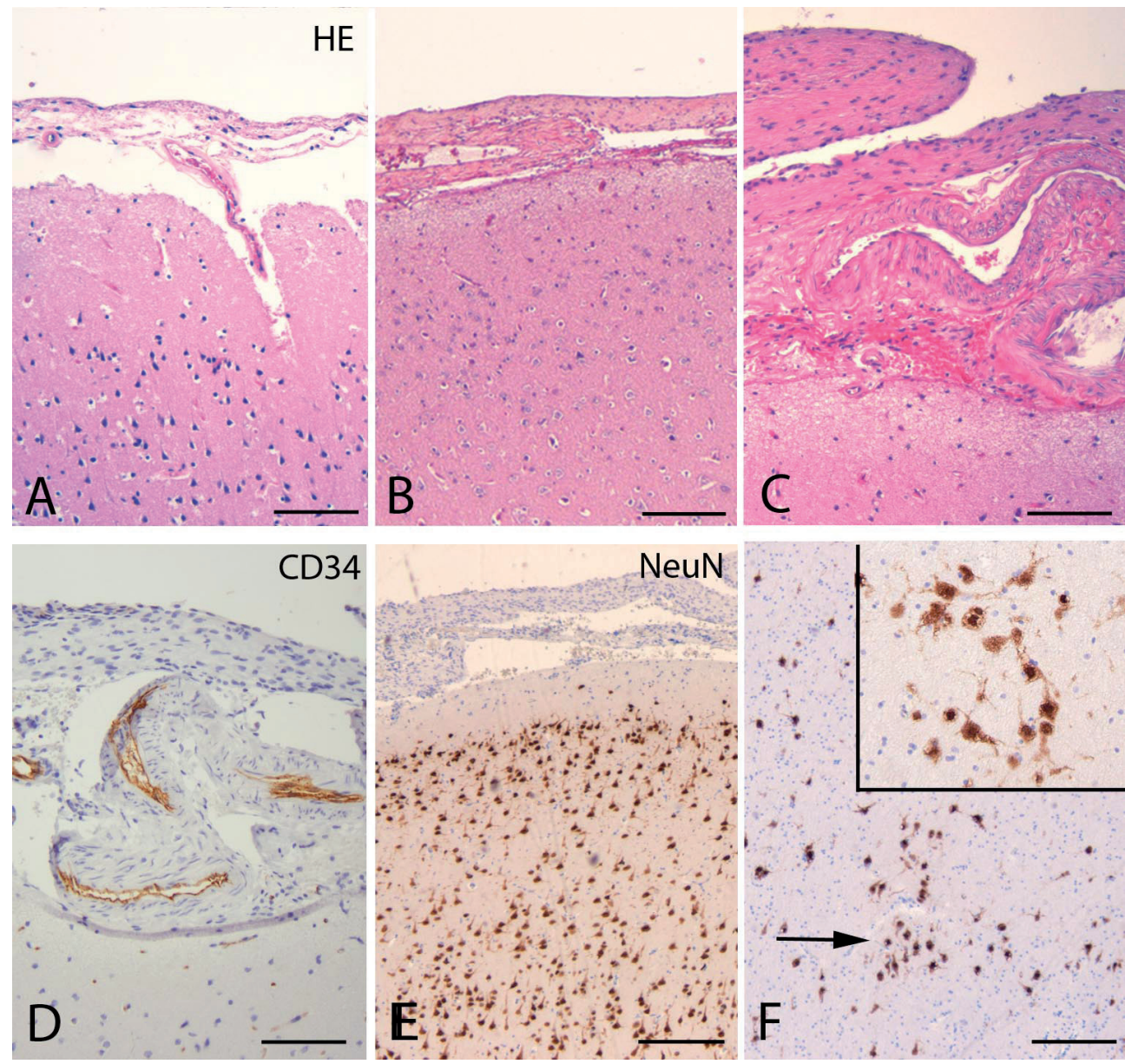

Supplementary figure 1. Histology.

A-B: Normal neocortex (HE, hematoxylin and eosin). B-C (HE) and D (CD34): neocortex from patient III with thick (focally fibrotic) leptomeninges and prominent vascular structures. E-F (NeuN): neocortex from patient III without cortical dyslamination, but with microscopic neuronal clusters (arrow; insert in F) and excess of neurons of normal morphology in the in deep white matter (isolated mild malformations of cortical development, mMCD Type 2). Scale bar: A, B, E, F: $320 \mu \mathrm{m}$; C, D: $160 \mu \mathrm{m}$. 

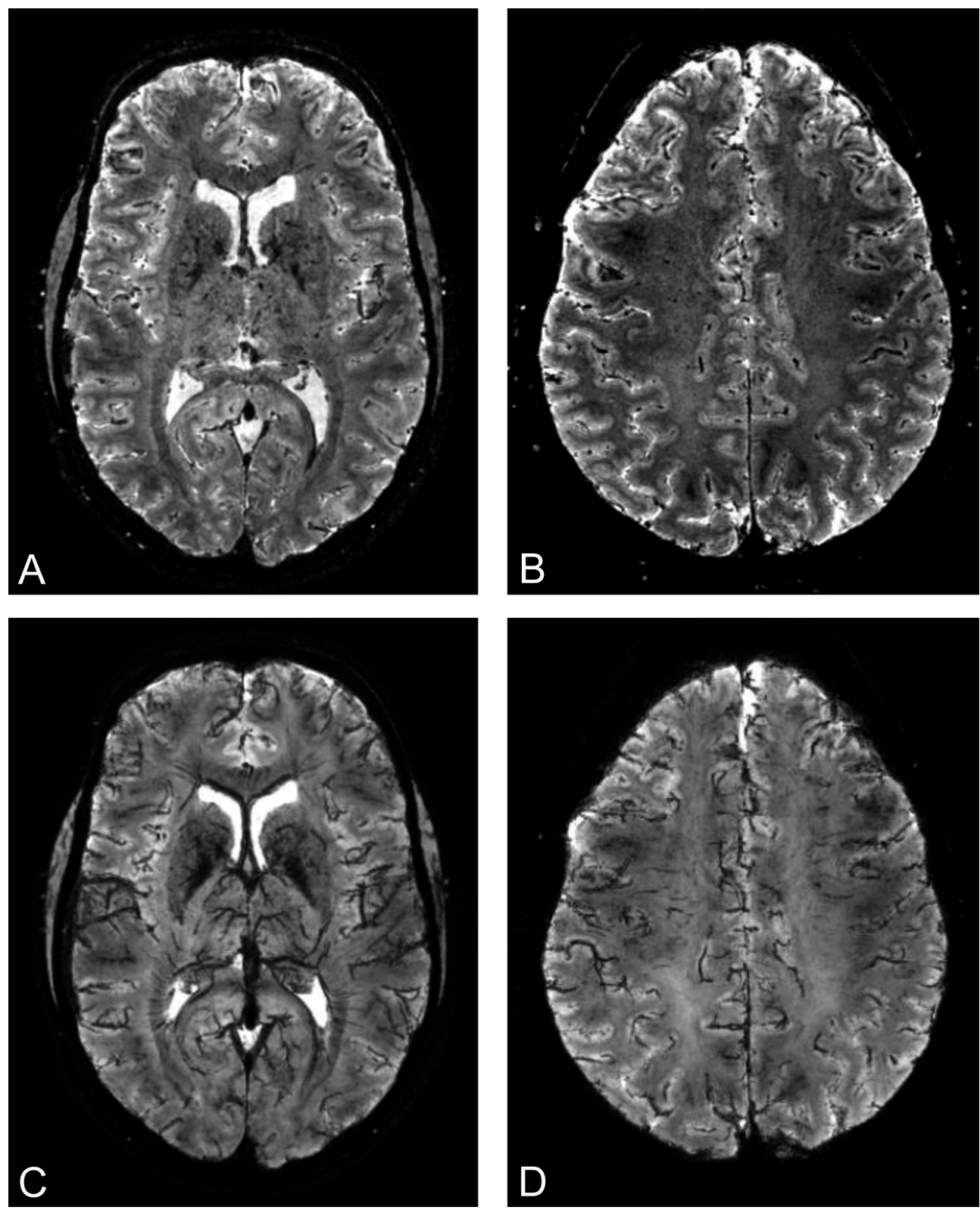

Supplementary figure 2 . Transverse $7 \mathrm{~T}_{2}{ }_{2}$ images of a healthy subject.

$T_{2}{ }^{*}$-weighted images, transverse slices parallel to the anterior commissure-posterior commissure line at the level of the foramen of Monroe (A) and at the level of the cingulate sulcus (B). Minimum intension projections with $5 \mathrm{~mm}$ slab thickness of the level of $A(C)$ and $B(D)$. These images show the normal appearance of venous cerebral vasculature in a healthy subject. 



\section{CHANGES IN VASCULAR DENSITY IN RESECTED TISSUE OF 97 PATIENTS WITH FOCAL CORTICAL DYSPLASI OR TSC-RELATED CORTICAL TUBERS}

Tim J. Veersema*, Andrew de Neef*, Jackelien van Scheppingen Cyrille H. Ferrier, Pieter van Eijsden, Peter H. Gosselaar, Peter C. van Rijen Wim G.M. Spliet, Kees P.J. Braun, Angelika Mühlebner ${ }^{\circ}$, Eleonora Aronica ${ }^{\circ}$ 
Recent studies suggested a possible association between malformations of cortical development and microvascular density. In this study we aimed to further elucidate the relation between microvascular density and cortical developmental abnormalities in a cohort of 97 patients with epilepsy and histologically proven mild malformation of cortical development (mMCD), focal cortical dysplasia (FCD) or tuberous sclerosis complex (TSC). Surgical tissue samples were analyzed with quantitative measures of vessel density, T-cell response, microglial activation and myelin content. Subsequently, the results were compared to an age- and localization matched control group. We observed an increase in microvasculature in white matter of TSC cortical tubers, which is linked to inflammatory response. No increase was seen in mMCD or FCD subtypes compared to controls. In mMCD/FCD and tubers, lesional cortex and white matter showed increased vascular density compared to perilesional tissues. Moreover, cortical vessel density increased with longer epilepsy duration and older age at surgery while in controls it decreased with age. Our findings suggest for that the increase in white matter vascular density might be pathology-specific rather than a consequence of ongoing epileptic activity. Increased cortical vessel density with age and with longer epilepsy duration in mMCD/FCD's and tubers, however, could be a consequence of seizures. 


\section{Introduction}

Epilepsy in young people is often caused by malformations of cortical development ${ }^{10}$, some of which are focal in nature and therefore termed focal cortical dysplasia (FCD). The International League Against Epilepsy (ILAE) published a new classification system for FCD which acknowledges three subtypes of FCD ${ }^{13}$. Type I is characterized by abnormal cortical layering, either by persistent radial micro-columnar architecture (la) or by a disruption of the horizontal lamination (Ib) or both (Ic). Type II is characterized by severe disorganization of the cortex and dysmorphic neurons (Ila), and balloon cells in addition (IIb). FCD type III refers to FCD in combination with other pathologies, such as hippocampal sclerosis (FCD type IIla), glial or glioneuronal tumors (FCD type IIIb), vascular malformations (FCD type IIIC) or any other principal lesion acquired during early life (FCD type IIId).

The most subtle architectural abnormalities in the FCD spectrum are classified as mild malformations of cortical development (mMCD), characterized by excessive number of neurons in the molecular layer (type 1) or white matter (type 2) without the presence of any other structural abnormalities - in contrast to FCD I types and II types ${ }^{13,14}$. mMCD as separate entity is subject to debate, but was included in the FCD ILAE classification, albeit as addendum ${ }^{13}$. Our group's recent surgical cohort study demonstrated differences between patients with mMCD and FCD histological diagnoses in clinical presentation and surgical outcome, in support of this clinical differentiation ${ }^{117}$.

A syndrome associated with refractory epilepsy that begins often in early childhood is Tuberous Sclerosis Complex (TSC). TSC is a genetic disorder caused by mutations in either the TSC1 or TSC2 gene expressed as hamartomas or benign tumors in several organ systems ${ }^{17,118}$. In the brain TSC can cause subependymal nodules, subependymal giant cell astrocytomas and cortical tubers which are typically associated with seizures. Tubers found in TSC are histologically often indistinguishable from FCD type $\mathrm{Ilb}^{13}$. Calcifications may differentiate tubers from FCD, but since over two-thirds do not exhibit this stigma, it is not a reliable distinguishing feature ${ }^{119}$.

In both isolated mMCD/FCD of all types, and in TSC, surgical removal of the epileptogenic lesion can result in seizure freedom ${ }^{10,120}$. Because many of these lesions are difficult to spot or outline on conventional MRI, or multiple in nature, it is often difficult to determine in which patients surgery is indicated. Therefore, it is of clinical importance to investigate associations between radiological features of FCD, histological characteristics and clinical outcomes. Recently 7 tesla MR imaging suggested a possible association between FCD and microvascular density ${ }^{121,122}$, which could possibly be used as diagnostic marker for dysplastic lesions. 
Evidence for increased vasculature in FCD lesions was found by Wintermark et al. using quantitative histological techniques and Arterial spin labelling (ASL)-MRI ${ }^{123}$. In contrast, Blauwblomme et al. found disorganized vascular structure but no increased density, and in their study PET-CT and ASL-MRI suggested lesional hypo- rather than hyperperfusion ${ }^{124}$. ASL MRI showed hypo and hyperfused tubers in patients with tuberous sclerosis, and while it was uncertain if hyperperfusion reflected epileptogenicity, a larger number of hyperperfused tubers in a patient was associated with increased seizure frequency ${ }^{125}$.

Uncertainty remains whether there is true increased vascular density in FCD or tubers, and if these changes would be an intrinsic etiological factor of epileptogenesis, part of the underlying malformation, or a result of aberrant neuronal activity. In this study we aimed to further elucidate the relation between microvascular density and cortical developmental abnormalities in a cohort of patients with histologically proven mMCD/FCD or TSC, and with possible etiopathological factors.

\section{Material \& Methods}

All patients who underwent epilepsy surgery between 2000 and 2012 at the University Medical Center Utrecht and had an initial histopathological report compatible with current mMCD/FCD subtypes were included. The pathological diagnosis was reviewed and established according to the currently available classification schemes for lesions associated with therapy-refractory epilepsy ${ }^{13,14}$. Patients with complex malformations (e.g. polymicrogyria, hemimegalencephaly) and associated cortical dysplasia (FCD III types) were omitted from the study.

Cortical tuber samples were added to the cohort by selecting patients from the database operated between 2002 and 2013 with confirmation of TSC through genetic testing and of whom tissues samples were available after resective epilepsy surgery.

In addition to TSC gene defects, (combinations of) multiple cortical or subependymal abnormalities, cutaneous stigmata or typical abnormalities in kidneys, heart or lung lead to a clinical TSC diagnosis, following the standard diagnostic criteria for TSC ${ }^{126}$.

The age- and localization-matched control group consisted of 14 autopsy cases. None of these patients had a history of seizures or other neurological diseases. All control samples were collected at the Department of (Neuro)Pathology, AMC, Amsterdam, The Netherlands.

Tissue was obtained and used in accordance with the Declaration of Helsinki and the AMC Research Code provided by the Medical Ethics Committee and approved by the science committee of the UMC Utrecht Biobank. 


\section{Tissue preparation and immunohistochemistry}

The tissue was carefully oriented, cut perpendicular to the pial surface, fixed overnight in $4 \%$ formaldehyde and routinely processed into liquid paraffin. Sections were cut at 4-6 $\mu \mathrm{m}$ with a microtome (Microm, Heidelberg, Germany), and mounted on positively charged slides (Superfrost + Menzel, Germany). Each specimen was histopathologically examined using hematoxylin \& eosin ( $\mathrm{H} \& \mathrm{E})$. The revised diagnosis was based on following commonly used immunohistochemical stainings: NeuN, SMI32, Vimentin, GFAP, and MAP2. The tissue samples were reclassified according to the 2011 ILAE classification system. Additional stainings were performed when necessary.

The immunohistochemical examinations for the quantitative analysis of all surgical specimens was performed using the following antibodies: CD34 (cluster of differentiation 34, 1:600, Qbend, Immunotech) endothelial staining for vascular density, CD3 (cluster of differentiation 3, 1:200, clone F7.2.38, DAKO) for T-cell presence, Cr3/43 (HLA-DP, DQ, DR antigen, DAKO) for microglia activation, GFAP (glial fibrillary acid protein, 1:4000, Dako, Glostrup, Denmark) for gliosis, MBP (myelin binding protein, 1:400 DAKO) for myelinization, NeuN (neuronal nuclei 1:100, clone A60, Chemicon, Billerica, MA, USA) for neurons, nonphosphorylated neurofilament H (SMI32) (1:1000, clone SMI32, Sternberger, Lutherville). The semi-quantitative analysis of blood brain barrier disruption was performed using albumin staining (Albumin Rabbit polyclonal 1:20,000 DAKO, Glostrup, Denmark). The slides were air dried overnight at $37^{\circ} \mathrm{C}$. All immunohistochemical stainings were performed with a Ventana semi-automated staining machine (Benchmark ULTRA; Ventana, Illkirch, France) and the Ventana DAB staining system according to the manufacturer's protocol.

\section{Quantitative measurements}

The CD34 stained slides were digitized using an IntelliSite Ultra Fast Scanner (Phillips healthcare Best, the Netherlands). Other stainings were digitized with an Olympus dotSlide system (vs 2.5, Olympus, Tokyo, Japan). In both scanners the magnification size was set on $100 x$ and pixels size was $0,64 \mu^{2}$. The slides were converted to Tagged Image File Format (TIFF) files. Every file was subsequently divided into evenly spread tiles. In Photoshop (Version: 2015.1.2) regions of interest (ROI's) with a median size of $0.83 \mathrm{~mm}^{2}$ ( range: 0.23 $6.5 \mathrm{~mm}^{2}$ ) were selected for the analysis. The selected ROI's were evenly spread within cortex, subcortical white matter (white matter was defined as being $>500 \mu \mathrm{m}$ away from the grey white matter border), lesion and, when available, perilesional tissue. Perilesional tissue was defined by the absence of aberrant cell forms such as dysmorphic neurons and balloon/ giant cells (for FCD II or TSC) and by normal cortical architecture (FCD subtypes and TSC). The lesional border in $\mathrm{mMCD}$ is less obvious, due to the subtle characteristics; perilesional tissue shows no excessive neurons in layer 1 or white matter. 
The available images were color deconvoluted according to the following RGB values: red: 0.26814753 , green: 0.57031375 and blue: 0.77642715 . The resulting 8 bit image was converted to a black and white mask (black: positively stained area, white: no staining). To discard background noise, a grey intensity value $(\mathrm{Gl})$ for each individual stain was selected.

\section{Quantitative analysis of cells and microvasculature}

To improve the accuracy and to avoid counting vessels or cells multiple times, a size range in $\mu \mathrm{m}^{2}$ was selected for each staining and no watershed filter was used. Microvessel count was performed using CD34 (Gl: 210, Pixel: 30-unlimited $\mu \mathrm{m}^{2}$ ), including capillaries and parenchymal arterioles. For the count of inflammatory cells, CD3 was used (GI: 160, Pixel: 10-unlimited $\mu \mathrm{m}^{2}$ ). Neuronal cell bodies were counted with $\mathrm{NeuN}$ (GI: 180, Pixel: 30-unlimited $\mu \mathrm{m}^{2}$ ). To analyze the amount of dysmorphic neuron, SMI32 was used (GI: 50, Pixel: 100-unlimited). All images were automatically processed via FIJ (ImageJ2, v64, open source) with a specifically developed macro based on Java programming language. Semi-automated quantification methods have been validated in our lab and described previously ${ }^{127}$.

Initial analysis revealed differences in vascular densities between tubers and controls that prompted further analysis of potential additional and explanatory contrasts or relationships regarding neuroinflammation, blood brain barrier dysfunction, and myelin content in tubers.

\section{Quantitative analysis of area size}

For area size analysis the total area of the ROI was determined using Image J. Subsequently the GI was set to remove background noise. Finally, field fraction of positively stained area was calculated. CR3/43 was used to measure microglial activation. To determine the number of reactive astrocytes, GFAP was used. Myelin content was examined using MBP. The $\mathrm{Gl}$ for all area size stainings was set on 180. The images were processed with a specific macro-based field fraction Java programming language in FIJI.

\section{Semi-quantitative scoring of balloon/giant cells and blood brain barrier damage}

The number of balloon/giant cells was scored on high power fields using an Olympus BX 50 microscope (each representing $1.081 \mathrm{~mm}^{2}$ ). The area of highest density was taken into account. We assessed the total coverage of abnormal cells within the grid area resulting in the following scores: I: 0-25\%, II: 25-50\%, III: 50-75\% and IV: 75-100\%. Blood brain barrier damage was also analyzed according to the same technique as used in earlier studies ${ }^{128-130}$. The intensity of the albumin staining was scored using a semi-quantitative scale ranging from 0-3 (0: negative, 1: weak, 2: moderate, 3: strong reactivity).

Quantitative and automated methods were not suitable for scoring balloon/giant cells or albumin extravasation due to non-specific staining patterns. 


\section{Clinical data}

Clinical patient data was collected for age at surgery, seizure duration in years and location of resected tissue (frontal, temporal, parietal or occipital).

\section{Statistical analysis}

Statistical analysis was performed with SPSS version 22 and 25 (IBM, PASW Statistics, USA). Descriptive statistics were used for the primary analysis: counts, mean, standard deviation. Vascular density counts were tested for normal distribution using Shapiro-Wilk test and for homogeneity of variances (scatterplot predicted versus observed). Test for normality (Shapiro-Wilk, $\mathrm{p}<0.001$ ) and homogeneity of variances (scatterplot predicted versus observed for vascular density and age) - failed for white matter vascular density. Therefore, we performed a natural logarithmic transformation for white matter vascular density. Grey matter and the log-transformed white matter vascular density showed normal distribution and homoscedasticity. T-test was used to assess differences in vascular densities between sex. We used multivariate analysis of covariance (MANCOVA) to test dependence of grey and white matter vascular density on age, region, epilepsy duration and consequently for differences between pathologies and for groupwise comparison of lesional versus perilesional densities. Multiple testing in post-hoc analysis was corrected for with Hochberg or Dunnett's test because of differences in sample sizes. Correlation was tested with Pearson's test, Kendell-Tau for correlation with semi-quantified balloon cell density and Spearman's rho for semi-quantifications of albumin leakage.

Paired-T test was performed to assess lesional versus perilesional vessel densities within the same patients.

\section{Results}

Patient characteristics are summarized in table 1. In total 97 patients with either $\mathrm{mMCD}$, FCD or TSC were included (31 mMCD, 52 FCD, 13 TSC). Median age at surgery was 14 years (range 3 months - 56 years). Three patients with FCD underwent a second surgical resection because of recurrent seizures.

\section{Control tissue vessel density}

As control group we included 14 cortical post-mortem samples (median age 15 years, range 2-42), sex: 9 male; location: 4 frontal, 8 temporal, 2 occipital). For 7 patients perilesional samples were also available (1 FCD lb, 1 FCD Ila, 2 FCD Ilb and 3 TSC). "Perilesional" was defined as cortex surrounding a main FCD II or TSC lesion without presence of giant cells or dysmorphic neurons. Subtle cortical abnormalities may be present. The group size of all pathologies included was highly variable, therefore we fused the groups into $\mathrm{mMCD}$ $(n=31)$, FCD I $(n=10)$, FCD Ila $(n=18)$, FCD Ilb $(n=28)$ and TSC $(n=13)$. Quantifications of vascular density are provided in table 2 . 
Table 1. Patient characteristics.

\begin{tabular}{|c|c|c|c|c|c|c|c|c|c|c|}
\hline \multirow{2}{*}{$\begin{array}{l}\text { Diagnosis } \\
\text { mMCD }\end{array}$} & \multirow{2}{*}{$\begin{array}{l}\text { Number } \\
\text { of } \\
\text { patients }\end{array}$} & \multirow{2}{*}{$\begin{array}{l}\text { Gender } \\
\delta / \% \\
\mathbf{1 3} / \mathbf{1 8}\end{array}$} & \multirow{2}{*}{$\begin{array}{l}\text { median age at } \\
\text { surgery } \\
\text { yrs. (range) }\end{array}$} & \multicolumn{4}{|c|}{$\begin{array}{l}\text { Localization } \\
\text { tissue samples } \\
\text { F T P O }\end{array}$} & \multirow{2}{*}{$\begin{array}{l}\text { Lesionala }^{a} \\
32\end{array}$} & \multirow{2}{*}{$\begin{array}{l}\text { Perilesio- } \\
\text { nala }^{\text {a }}\end{array}$} & \multirow{2}{*}{$\begin{array}{l}\text { median } \\
\text { epilepsy } \\
\text { duration yrs. } \\
\text { (range) }\end{array}$} \\
\hline & & & & 9 & 17 & 4 & 1 & & & \\
\hline $\begin{array}{l}\text { mMCD } \\
\text { type } 1\end{array}$ & 7 & $3 / 4$ & $32(19-48)$ & 4 & 3 & 0 & 0 & 8 & 0 & $16(7-32)$ \\
\hline $\begin{array}{l}\text { mMCD } \\
\text { type } 2\end{array}$ & 24 & $10 / 14$ & $19(1-46)$ & 5 & 14 & 4 & 1 & 24 & 0 & $7(1-30)$ \\
\hline FCD I & 9 & $3 / 6$ & $\begin{array}{l}13 \text { (7 months - } \\
56 \text { yrs.) }\end{array}$ & 3 & 5 & 1 & 0 & 9 & 1 & $5(1-42)$ \\
\hline FCD la & 4 & $2 / 2$ & $\begin{array}{l}2 \text { (7 months - } \\
56 \text { yrs. }\end{array}$ & 0 & 3 & 1 & 0 & 4 & 0 & $2(1-42)$ \\
\hline FCD Ib & 4 & $1 / 3$ & $14(5-40)$ & 2 & 2 & 0 & 0 & 4 & 1 & $7.5(2-23)$ \\
\hline FCD IC & 1 & $0 / 1$ & 32 & 1 & 0 & 0 & 0 & 1 & 0 & 23 \\
\hline FCD II & 44 & $25 / 19$ & $\begin{array}{l}13 \text { (3 months } \\
-46)\end{array}$ & 32 & 7 & 4 & 1 & 46 & 3 & $7.5(0-37)$ \\
\hline FCD Ila & 17 & $11 / 6$ & $\begin{array}{l}13 \text { (3 } \\
\text { months-45 yrs.) }\end{array}$ & 12 & 4 & 1 & 0 & 18 & 1 & $6(0-37)$ \\
\hline FCD IIb & 27 & $14 / 13$ & $\begin{array}{l}13 \text { (8 } \\
\text { months-41 yrs.) }\end{array}$ & 20 & 3 & 3 & 1 & 28 & 2 & $9(1-36)$ \\
\hline TSC & 13 & $7 / 6$ & $\begin{array}{l}9(10 \\
\text { months-47 }\end{array}$ & 9 & 4 & 0 & 0 & 14 & 3 & $5(1-35)$ \\
\hline total & 97 & $48 / 49$ & $\begin{array}{l}14 \text { (3months- } 56 \\
\text { yrs. }\end{array}$ & 53 & 33 & 9 & 2 & 101 & 7 & $8(0-42)$ \\
\hline
\end{tabular}

Overview of the patients, gender distribution ( $\delta=$ male, $q=$ female) lesion type and location, epilepsy duration in years. The different pathology types are mild malformation of cortical development (mMCD), Focal cortical dysplasia (FCD) and Tuberous Sclerosis Complex (TSC). The different brain regions are Frontal $(F)$, Temporal $(T)$, Parietal $(P)$, Occipital $(O)$.

${ }^{a}$ Note, for certain patients only lesional, perilesional tissue or both were available for analysis. The sum of lesional and perilesional samples exceeds the number of patients due to three patients that underwent surgery twice and seven patients with both lesional and perilesional samples available for analyses. 
In post-mortem control tissue we measured a mean vascular density of $154.3 \pm 40.8 / \mathrm{mm}^{2}$ in cortex and in white matter a median density of $65.2 / \mathrm{mm}^{2}$ (range $44.6-118.3$ ). To further characterize vascular density in controls, we first compared vascular density between men and women and found no significant differences in cortex $(t(12)=1.026, p=0.325)$, or white matter $(t(12)=1.346, p=0.195)$.

White matter and cortical vascular density were correlated $(r=0.551 ; p=0.041)$.

In multivariate regression analysis we found a significant association between vascular densities and age $(F(2,9)=5,252 ; p=0.031$; Wilk's $\lambda=0.461)$ and brain region $(F(4,18)=3.988$; $p=0.017$; Wilk's $\lambda=0.280$ ). Corrected for region, there was a negative association between age and cortical vessel density $(F(1)=11.512 ; p=0.007 ; r(14)=-.704, p=0.005)$ (figure 1 and figure $2 a)$ but not between age and white matter vessel density $(F(1)=2.289 ; p=0.161)$ (figure 1 and figure $2 \mathrm{c}$ ). There was a significant effect of region on cortical vascular density for cortex $(F(2)=11.024, p=0.03)$, correcting for age. Occipital regions $\left(221.0 \pm 23.0 / \mathrm{mm}^{2}\right)$ had highest cortical vascular density followed by frontal $\left(168.9 \pm 16.8 / \mathrm{mm}^{2}\right)$ and temporal regions (130.4 $\pm 29.2 / \mathrm{mm}^{2}$ ) (no parietal control samples).

\section{Patient tissue vessel density}

In the analysis of vascular density in lesional tissue of 97 patients no sex difference was detected either (cortex T-test $\mathrm{p}=0.771$; white matter T-test $\mathrm{p}=0.917$ ).

Corrected for age, there were significant regional differences in cortex $(F(3)=8.021, p<0.0005)$ but not in white matter $(F(3)=1.482, p=0.224)$ vascular density. Highest cortical vascular density was found occipitally (mean $184.57 \pm 57.02$ vessels per $\mathrm{mm}^{2}$ ), followed by frontal lobe (mean 162.24 \pm 40.36 ), parietal lobe (mean $147.41 \pm 20.53$ ) and temporal lobe cortex (mean 122.53 \pm 43.60 ). In post-hoc analysis - omitting age as co-variate-there were significant differences between frontal and temporal (LSD, $p<0.0005$, Hochberg $p<0.0005)$ and between temporal and occipital lobe cortical vascular densities (LSD, $p=0.041$, Hochberg $=0.220$ ).

Corrected for region, vessel density in lesional samples was positively associated with age at surgery $(F(2,93)=7.163 p=0,001)$ and with epilepsy duration $(F(2,93)=5.400, p=0.006)$ in

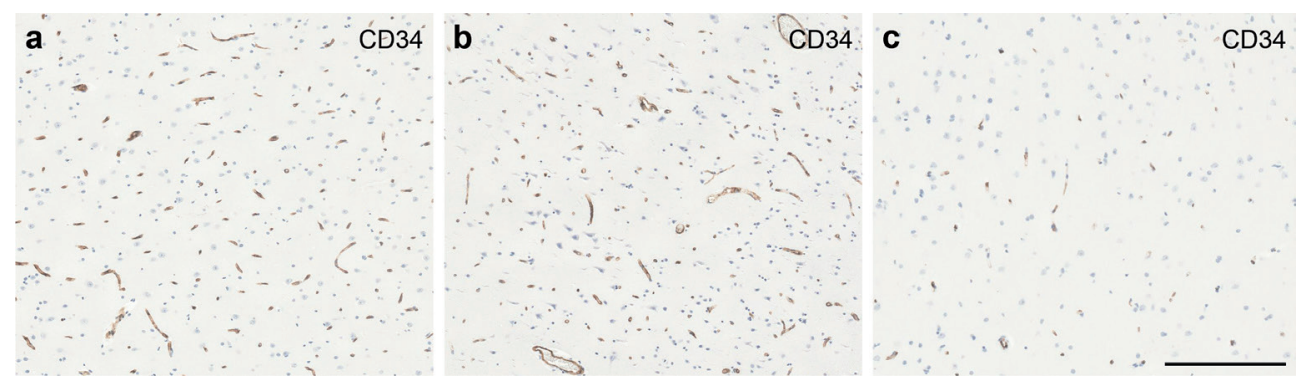

Figure 1. Cortical vascular density in healthy controls declines with age, $p=0,007$. The temporal region of a 2-year-old (a), 17-year-old (b) and 42-year-old subject (c) scale bar in $d=100 \mu \mathrm{m}$. 

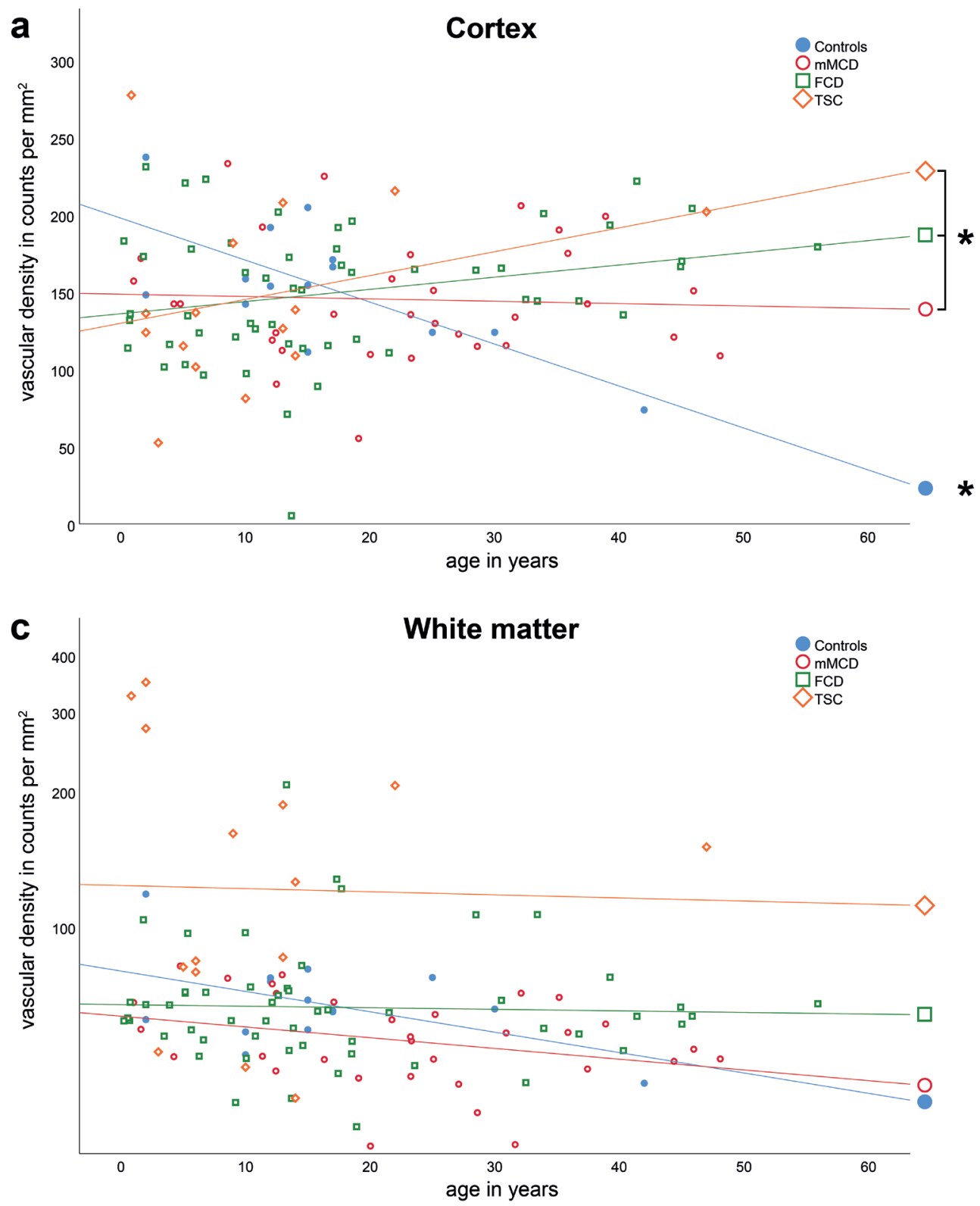

Figure 2. Vascular density in cortex $(\mathbf{a}, \mathbf{b})$ and white matter $(\mathbf{c}, \mathbf{d})$ in controls, $\mathrm{mMCD}, \mathrm{FCD}$ and TSC samples in relation to age at surgery $(\mathbf{a}, \mathbf{c})$ and epilepsy duration $(\mathbf{b}, \mathbf{d})$. There is no relation between age and epilepsy duration and vascular density in white matter $(\mathbf{c}, \mathbf{d})$, while in cortex density declined with age in controls (a) and increased with age and epilepsy duration in lesions, most pronounced in TSC $(\mathbf{b}, \mathbf{d})$. 
b

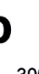

300

$\diamond$

250

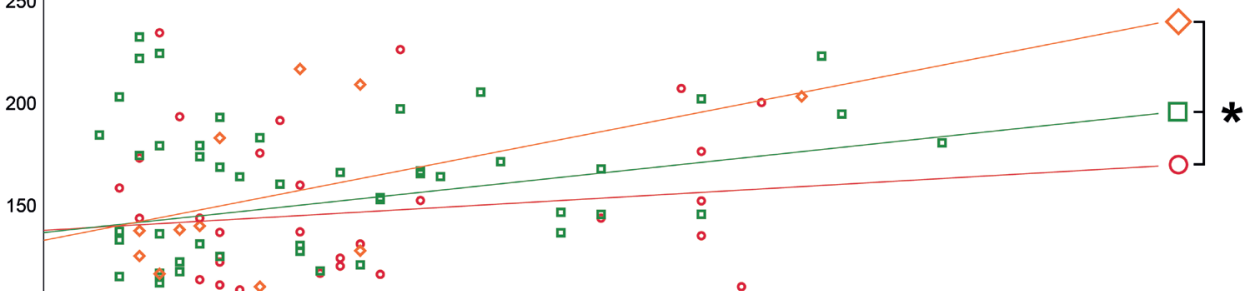

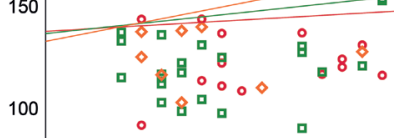

口

$50 \diamond$

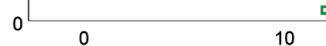

epilepsy duration in years

d

400

300

300

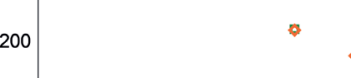

Cortex

$\mathrm{O} \mathrm{mMCD}$

그미

$\checkmark$ TSC

White matter

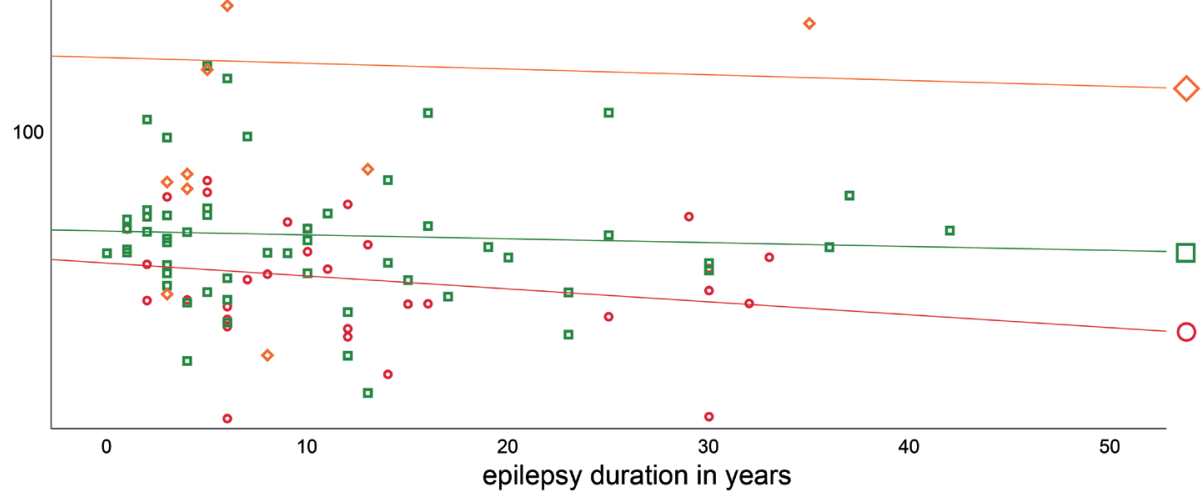


Table 2. Vascular densities in cortex and white matter.

\begin{tabular}{|c|c|c|c|c|c|c|c|c|}
\hline & \multicolumn{4}{|c|}{ vascular density in cortex, count $/ \mathrm{mm}^{2}$} & \multicolumn{4}{|c|}{ vascular density in white matter, count $/ \mathrm{mm}^{2}$} \\
\hline & mean $\pm S D$ & median & range & $\mathrm{p}$ & mean $\pm S D$ & median & range & $\mathrm{p}$ \\
\hline controls & $\begin{array}{l}154.3 \\
\pm 40.8\end{array}$ & 154.1 & $\begin{array}{l}73.7- \\
237.2\end{array}$ & $0.057^{\mathrm{a}}$ & $69.1 \pm 17.4$ & 65.2 & 44.6-118.3 & $0.866^{\mathrm{a}}$ \\
\hline Lesional & $\begin{array}{l}147.2 \\
\pm 44.4\end{array}$ & 142.3 & $\begin{array}{l}5.51- \\
277.4\end{array}$ & & $76.3 \pm 51.1$ & 62.8 & $32.2-348.7$ & \\
\hline perilesional & $\begin{array}{l}116.7 \\
\pm 39.4\end{array}$ & 99.0 & $\begin{array}{l}73.7- \\
192.0\end{array}$ & & $69.0 \pm 23.3$ & 65.8 & 41.4-99.2 & \\
\hline $\begin{array}{l}\text { frontal } \\
\text { (controls) }\end{array}$ & $\begin{array}{l}168.9 \\
\pm 16.8\end{array}$ & 164.7 & $\begin{array}{l}154.3- \\
191.8\end{array}$ & $0.03^{\mathrm{b}}$ & $65.6 \pm 10.5$ & 66.8 & $51.7-76.9$ & $0.579^{\mathrm{b}}$ \\
\hline $\begin{array}{l}\text { temporal } \\
\text { (controls }\end{array}$ & $\begin{array}{l}130.4 \\
\pm 29.2\end{array}$ & 133.1 & $\begin{array}{l}73.7- \\
166.3\end{array}$ & & $66.0 \pm 11.7$ & 65.1 & $44.6-80.5$ & \\
\hline $\begin{array}{l}\text { parietal } \\
\text { (controls) }\end{array}$ & - & - & - & & - & - & - & \\
\hline $\begin{array}{l}\text { occipital } \\
\text { (controls) }\end{array}$ & $\begin{array}{l}221.0 \\
\pm 23.0 \\
\end{array}$ & 204.7 & $\begin{array}{l}204.7- \\
237.2\end{array}$ & & $88.6 \pm 42.0$ & 88.6 & $58.9-118.3$ & \\
\hline $\begin{array}{l}\text { frontal } \\
\text { (lesional) }\end{array}$ & $\begin{array}{l}162.2 \\
\pm 40.4\end{array}$ & 167.4 & $\begin{array}{l}71.0- \\
233.1\end{array}$ & $<0.0005^{\mathrm{b}}$ & $84.2 \pm 56.9$ & 67.7 & $32.2-348.7$ & $0.224^{b}$ \\
\hline $\begin{array}{l}\text { temporal } \\
\text { (lesional) }\end{array}$ & $\begin{array}{r}124.5 \\
\pm 43.8 \\
\end{array}$ & 123.2 & $\begin{array}{l}5.3- \\
277.4\end{array}$ & & $67.2 \pm 48.6$ & 56.3 & $32.4-325.3$ & \\
\hline $\begin{array}{l}\text { parietal } \\
\text { (lesional) }\end{array}$ & $\begin{array}{l}140.8 \\
\pm 28.5\end{array}$ & 142.3 & $\begin{array}{l}81.2- \\
181.7\end{array}$ & & $66.9 \pm 16.7$ & 62.1 & $48.5-106.5$ & \\
\hline $\begin{array}{l}\text { occipital } \\
\text { (lesional) }\end{array}$ & $\begin{array}{l}184.6 \\
\pm 57.0\end{array}$ & 184.6 & $\begin{array}{l}144.3- \\
224.9\end{array}$ & & $54.0 \pm 5.0$ & 54.0 & $50.5-57.6$ & \\
\hline $\begin{array}{l}\text { mMCD } \\
\text { (lesional) }\end{array}$ & $\begin{array}{l}145.1 \\
\pm 39.6\end{array}$ & 139.0 & $\begin{array}{l}55.3- \\
233.1\end{array}$ & $0.495^{\mathrm{a}}$ & $57.0 \pm 12.7$ & 56.2 & $32.2-81.8$ & $<0.0005^{a}$ \\
\hline $\begin{array}{l}\text { FCD I } \\
\text { (lesional) }\end{array}$ & $\begin{array}{l}143.2 \\
\pm 41.7 \\
\end{array}$ & 135.2 & $\begin{array}{l}89.0- \\
220.6\end{array}$ & & $59.5 \pm 8.3$ & 61.8 & $44.8-71.1$ & \\
\hline $\begin{array}{l}\text { FCD Ila } \\
\text { (lesional) }\end{array}$ & $\begin{array}{r}153.3 \\
\pm 58.3 \\
\end{array}$ & 168.2 & $\begin{array}{l}5.3- \\
231.0\end{array}$ & & $60.7 \pm 11.3$ & 62.4 & $35.6-77.2$ & \\
\hline $\begin{array}{l}\text { FCD IIb } \\
\text { (lesional) }\end{array}$ & $\begin{array}{l}149.5 \\
\pm 30.9\end{array}$ & 151.4 & $\begin{array}{l}96.3- \\
221.7\end{array}$ & & $73.6 \pm 11.3$ & 62.4 & $35.6-77.2$ & \\
\hline TSC & $\begin{array}{l}143.0 \\
\pm 59.1\end{array}$ & 131.3 & $\begin{array}{l}52.5- \\
277.4\end{array}$ & & $\begin{array}{l}146.2 \\
\pm 98.2\end{array}$ & 106.8 & $41.3-348.7$ & \\
\hline
\end{tabular}

a Mancova, corrected for age and region

${ }^{\mathrm{b}}$ Mancova, corrected for age

mMCD: malformation of cortical development. FCD: focal cortical dysplasia. TSC: tuberous sclerosis complex 
cortex (figure $2 a, b$ ), but not in white matter (figure $2 c, d$ ). The positive relation between age and cortical vascular density was more pronounced in TSC (figure $2 b$ ).

Corrected for age and region we found no significant vascular density differences in groupwise comparison between lesional samples and controls $(F(2,104)=0.982, p=0.399$, Wilk's $\lambda=0.928$ ).

Groupwise comparison between lesional $\left(147.2 \pm 44.4 / \mathrm{mm}^{2}\right)$ and perilesional $(116.7 \pm 39.4$ $/ \mathrm{mm}^{2)}$ cortical density showed a trend for higher density of lesional tissues, regardless of age and region $(F(1)=4.025, p=0.048)$. No difference was found in white matter vascular densities $(F(1)=0.152, p=0.698)$.

In a pairwise analysis of the seven patients of whom both lesional and perilesional samples were available we found significantly higher vascular densities in the lesional cortex $(t(6)=3.156, p=0.02$ ) (figure $3 a$ ) and in the lesional white matter $(t(6)=2.733, p=0.034$ ) (figure $3 d$ ) compared to perilesional tissue (lesional cortex $160.2 \pm 30.4$, perilesional cortex 116.7 $\pm 39.4 / \mathrm{mm}^{2}$; lesional white matter median 96.7, range 52.9-206.0, perilesional white matter median 65.8 range 41.4-99.2 $/ \mathrm{mm}^{2}$ ). In all individual cases lesional cortex had a higher vascular density than the neighboring perilesional tissue. In five of seven white matter pairs, vascular density was higher in the lesional tissue.

We compared vessel densities between the different pathologies with grouping of $\mathrm{mMCD}$ (type 1 and 2), FCD I (a, b and c), FCD Ila, FCD IIb and cortical tubers in TSC. Corrected for age and region there were significant differences between pathologies in lesional vascular density $(F(10,184)=5.478, p<0.005$, Wilk's $\lambda=0.594)$. This is due to significant differences in white matter vessel densities across pathologies $(F(5)=9.569, p<0.0005)$. No differences were found in cortical density $(F(5)=0.884, p=0.495)$. Post-hoc testing showed that only TSC samples (median 106.8, range 41.3-348.7 $\mathrm{mm}^{2}$ ) had a significantly higher white matter vascular density compared to control samples (median 65.2, range 44.6-118.3 mm², Dunnett, $\mathrm{p}<0.0005$ ) (figure 4a). Perilesional samples were excluded from these analyses.

There was no correlation between balloon cell density and vessels in FCD IIb and TSC (Kendall-tau, grey matter $\mathrm{p}=0.679$, white matter $\mathrm{p}=0.196$ ).

FCD Ilb and cortical tubers have identical histological features. However, we observed a difference in white matter vascular density between FCD IIb and tubers. Corrected for age, white matter vascular density was significantly different $(F(2,36)=9.683, p<0.0005$, Wilk's $\lambda$ $=0.650$ ), with higher densities in TSC (median 106.8 range 41.3-348.7 $\mathrm{mm} 2$ ) compared to FCD Ilb (median 62.4, range 35.6-77.2) $(F 1)=16.481 \mathrm{p}<0.0005)$. Cortical vessel density did not differ between FCD IIb and tubers. 

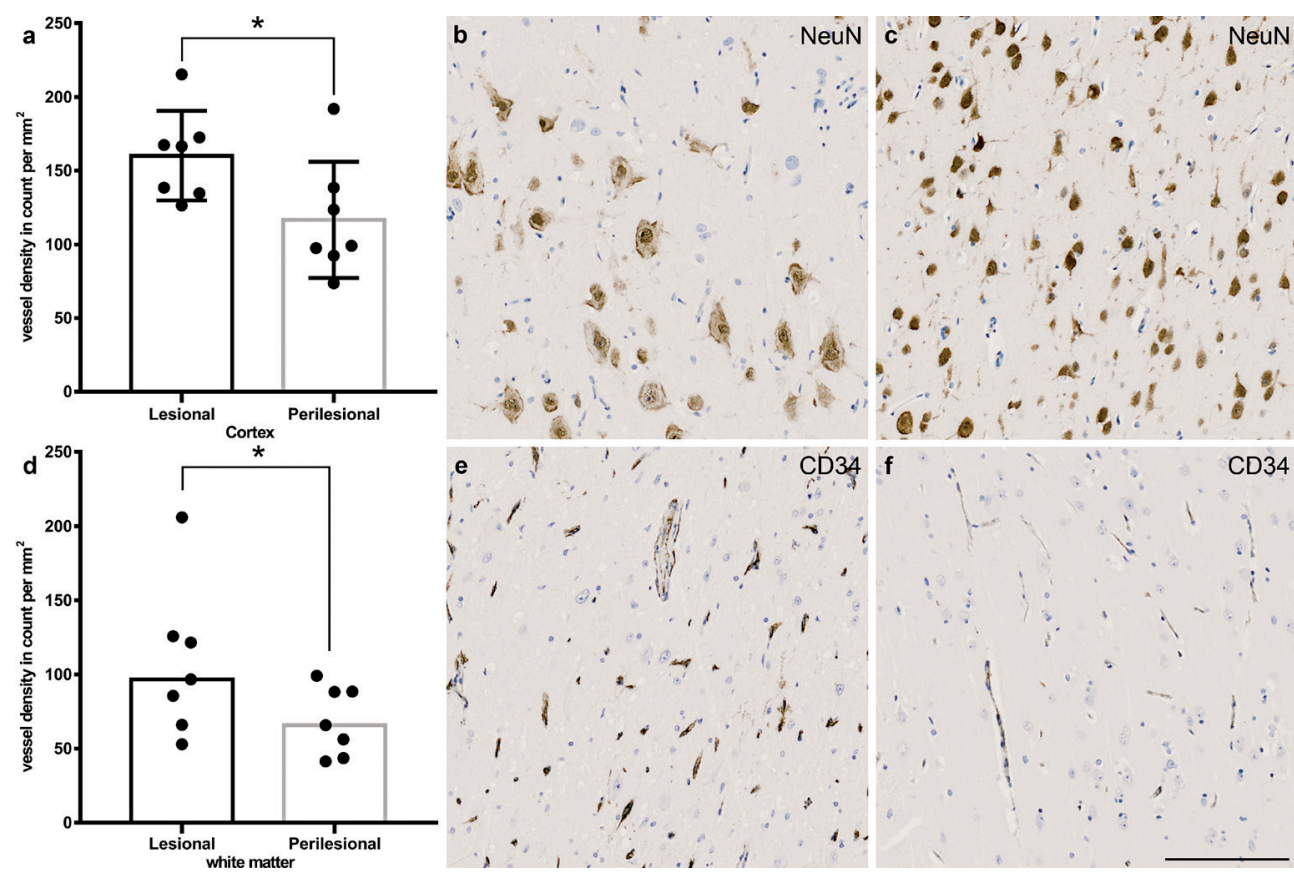

Figure 3. Lesional vs perilesional vascular densities in cortex (a) and white matter (d). Significant difference in vessel density (per $\mathrm{mm}^{2}$ ) in pairwise analysis between lesional and perilesional grey matter $(p=0.02)$ (a, mean and $1 \mathrm{std}$ for bar and whiskers), and white matter $(p=0.034)$ ( $\mathbf{d}$, median for bar, non-parametric data). A characteristic feature of type llb focal cortical dysplasia is the presence of dysmorphic neurons (b) (as shown by NeuN staining, in grey matter). Perilesional grey matter (c) does not contain dysmorphic neurons. Lesional vessel density in FCD Ilb (e) (as shown by CD34 staining) compared to perilesional vessel density (f) in grey matter. Scale bar in $f=100 \mu \mathrm{m}$ and also applies to $\mathbf{b}, \mathbf{c}, \mathbf{e}, \mathbf{f}$.

Because cortical tubers of the TSC cohort showed a significantly higher number of vessels in white matter compared to controls, we continued to examine the tuber samples with different stainings which were mainly focused on the role of inflammation and the damage of the blood brain barrier (BBB). Because of the lack of differences between the FCD and control groups, we did not examine more stainings within FCD or mMCD groups.

TSC samples showed a positive correlation between vascular density in white matter and microglial activation ( $\mathrm{Cr} 3 / 43$ positivity, $r(10)=0.716, \mathrm{p}=0.02$ ) (figure $4 \mathrm{e}$ ).

White matter vascular density tended to correlate with inflammatory cell response (CD3 activity, $r(10)=0.563, p=0.09)$.

Inflammatory cell response significantly correlated with microglial activation in white matter $(r(10)=0.823, p=0.003)$. We did not find a correlation between vascular density and myelin content. 


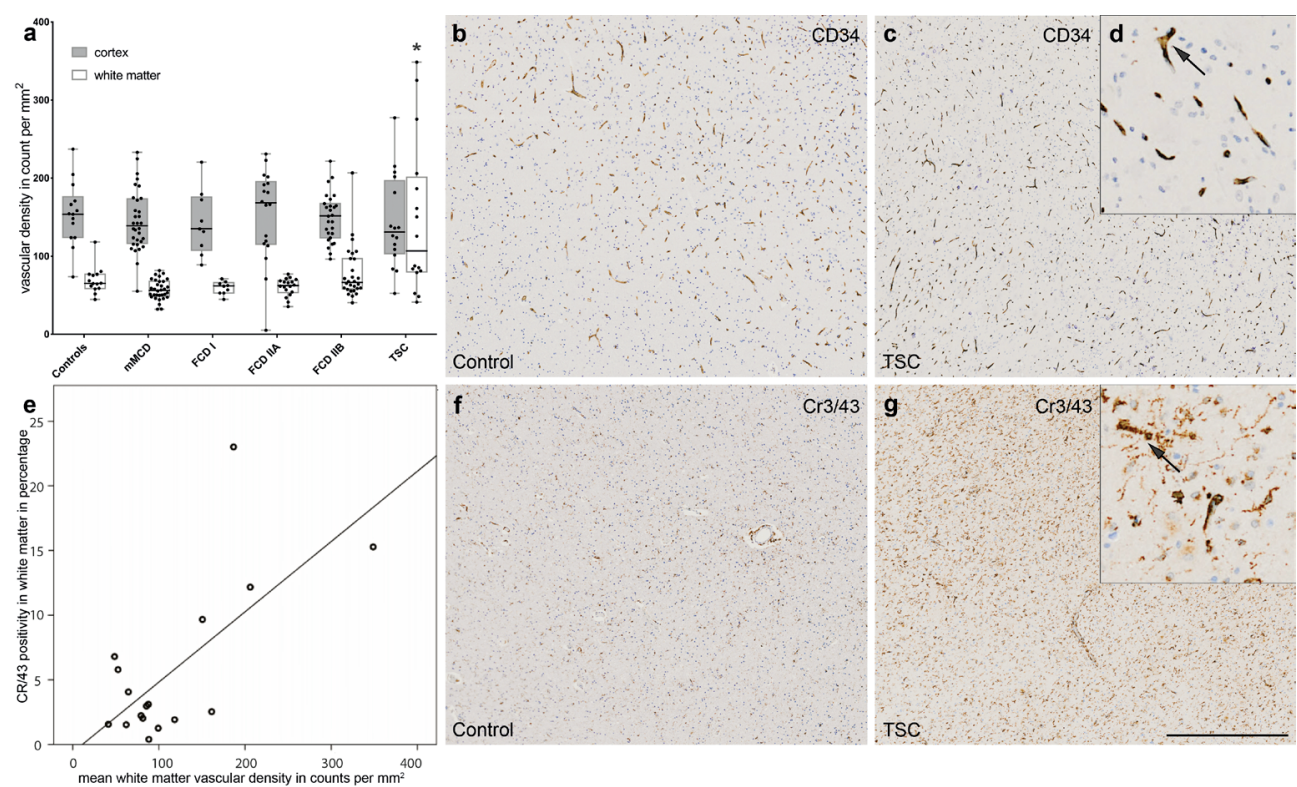

Figure 4. Histological subgroups, vascular density and microglia activation. a Increased white matter vessel density in TSC compared to control in white matter, $\mathrm{p}<0.001$ (box shows median and $25^{\text {th }}$ and $75^{\text {th }}$ percentile, whiskers min-max). Vessel density in white matter in a healthy control (b) and a cortical tuber in a patient with TSC (c). micro-vasculature is clearly visible with CD34 endothelial staining (d). increased vessel density is accompanied with increased microglia activation in a tuber (e), $p<0.05$. Microglial activation in control (f) vs TSC (g). Microglial activation can clearly be seen with $\mathrm{Cr} 3 / 43$ staining (h). Scale bar in $\mathrm{g}=100 \mu \mathrm{m}$ and also applies to $\mathbf{b}-\mathbf{c}$ and $\mathbf{f}-\mathbf{g}$.

We then performed a semi-quantitative analysis of the BBB leakage, with albumin as marker. Albumin deposits were observed in grey and white matter in all TSC samples (figure 5), but there was no significant correlation between quantities of extracellular albumin and vascular density.

\section{Discussion}

This study provides the first description of relationship between microvascular density and cortical developmental abnormalities in a large cohort of patients with either cortical dysplasia or cortical tubers in TSC.

We found that cortical and white matter vascular density is increased in FCD and tubers compared to the surrounding perilesional cortex, irrespective of age. While in healthy controls cortical vascular density decreased with age, in epilepsy patients with FCD or tubers density appeared to increase with age and with longer epilepsy duration. No timerelation was seen in white matter vascular density. Tubers had an increased microvascular 

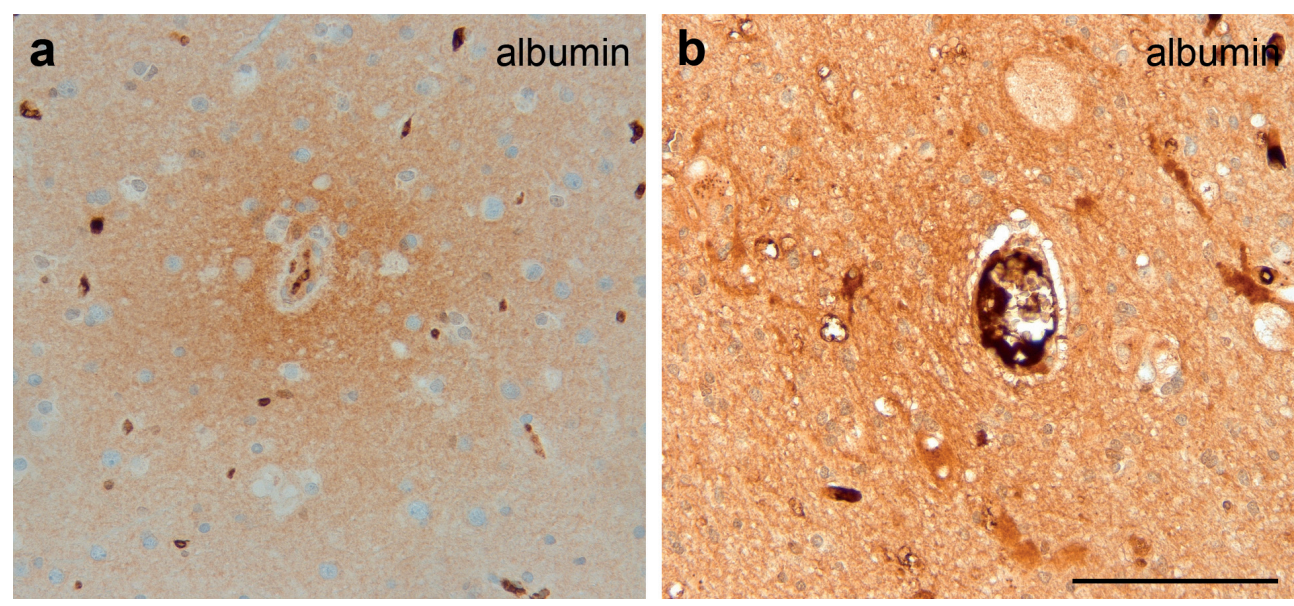

Figure 5. Albumin deposits in TSC. a Deposits of albumin in grey matter indicating damage to the blood brain barrier. B similar deposits can be seen in white matter Scale bar in $\mathbf{b}=100 \mu \mathrm{m}$ and also applies to $\mathbf{a}$.

density in white matter compared to age-matched controls. The latter was associated with microglial activation and an increased number of inflammatory cells.

In both healthy subjects and in patients there were significant regional differences in cortical vascular density. The highest densities were found in the occipital region, followed by frontal, parietal and temporal locations. This is likely linked to the regional differences in brain metabolism ${ }^{131,132}$. Moreover, several studies have indicated that the same area consists of a higher numbers of neurons which are necessary for the visual processing ${ }^{133-136}$. However, the high level of variance amongst the existing studies does not allow clear-cut conclusions concerning topography of vascular density. As described by Riddle et al., a more robust analysis of the different regions in the brain, with a standardized method is needed, to see if this finding is consistent in the general population ${ }^{137}$.

Furthermore, our data suggests not only differences in vascular density between brain regions, but also variation with age due to constant remodeling of the brain, a process thought to be mainly based on the functional needs of the brain ${ }^{113,138}$.

Although there is not yet a clear consensus on the effect of age on vascular density, a decline has been observed in several studies ${ }^{137}$. Correspondingly, we found a decrease in cortical vascular density with advancing age in controls.

In contrast to normal cortex, in resected malformations of cortical development - most pronounced in cortical tuber - we observed an increase in vascular density with ageing. This could suggest that certain processes in lesional or epileptogenic cortex drive ongoing 
angiogenesis and increasing vascularity, rather than that the higher vessel density is static and reflects and intrinsic part of the developmental abnormality. Moreover, in contrast to cortical vascular density, vessel density in white matter was related to pathology specific processes. Compared to controls, increased density was only observed in tubers and not in FCD subtypes.

We did not perform rater variability analysis for the $\mathrm{mMCD} / \mathrm{FCD}$ reclassification, but prior research has shown good intra-observer reliability, good interobserver agreement for FCD II subtypes and moderate agreement for non-FCD and FCD la en Ib types in the evaluation according to the 2011 ILAE classification ${ }^{139}$. However, we acknowledge that no such attempt has been made for the mMCD so far and therefore this group remains rather heterogeneous, albeit with distinct clinical features ${ }^{117}$.

During brain development, early migration in particular, neuronal progenitor cells associate with blood vessels of the vascular plexus in the (sub)ventricular zone-which develops relatively early in embryogenesis,-and migrate abluminally to their target location in the cortex. This vasophilic migration mechanism is one of several involved in cortex formation ${ }^{140,141}$. Analogously, neuroblast association with microvessels is a phenomenon that is nowadays widely accepted as one of the main drivers of the massive infiltration in human gliomas, proving that vessel sprouting and the mode of neuronal migration can be re-activated any time ${ }^{142}$.

Interestingly is however that severe cortical disorganization is not invariably associated with aberrant vessel architecture or density, as shown in studies investigating the development of cortical microvasculature in reeler mice, exhibiting normal vasculature patterns and abnormal, inverted, laminar position of cortical neurons ${ }^{141}$. Nevertheless the observation that newly formed neurons and glia cells strongly associate with the vascular plexus and are involved in migration processes suggest there is possible interaction between developing vasculature and cortex formation, which could have pathomechanistic significance ${ }^{141}$.

In tubers, TSC1 or TSC2 gene dysfunction causes disinhibition of mTOR activity, which leads to upregulation of HIF-1a expression, increased vascular endothelial growth factor (VEGF) production and angiogenesis ${ }^{143}$. Tubers are dynamic processes and serum VEGF levels in TSC patients are related to proliferation of lesions. VEGF production appears to be secondary to mTOR activation and is rapidly reversed by rapamycin, a mTOR inhibitor ${ }^{118,143}$. This is also compatible with the observation of increasingly high vascular densities at older age.

BBB dysfunction is known to occur as a result of epileptic seizures and reciprocally plays a role in epileptogenesis and can sustain or aggravate epilepsy ${ }^{144}$. Almost all TSC samples showed evidence of BBB dysfunction, with extravasation of albumin. In addition, there 
was an increase of T-cells that are not able to infiltrate the cortex and white matter without leakage of the $\mathrm{BBB}^{145}$. However, we did not find a correlation between quantifications of microvascular density and BBB leakage.

Tubers exhibit complex activation of pro-inflammatory pathways, including chemokines, interleukin-1 $\beta$, HMGB1 (high-mobility-group-box-1), TLR (toll-like-receptor), RAGE (receptors for advanced glycation end products) and complement mediated pathways ${ }^{118}$. Local seizure activity triggers the release of a multitude of vasoactive substances which alter neurovascular coupling and can cause oxidative stress which both contribute to BBB dysfunction. In addition to promoting angiogenesis, VEGF increases BBB permeability through direct induction of inflammation ${ }^{146}$ and activation of matrix metalloproteases which break vessel walls and degrade tight junctions ${ }^{147}$.

Alternatively, extravasated serum proteins are phagocytized by activated microglia and astrocytes $^{148}$, elicit an inflammatory response ${ }^{149}$ and in turn possibly drive angiogenesis. In TSC samples we found a correlation between white matter vascular density and microglial activation, which co-occurred with T-cell activation. This supports a relation between inflammation and the observed angiogenesis.

Even though angiogenic processes and BBB dysfunction in epilepsy are generally considered detrimental for brain tissue and progression of disease, these could also be seen as protective mechanisms. Fast VEGF release, through FLK-1/AKT pathways, and activation of nitrous oxide-synthase, mediates vasodilation through nitrous oxide release ${ }^{150,151}$. In combination with increased vasculature these processes help to ensure adequate perfusion in tissue with increased metabolic demand due to epileptic activity.

We had hypothesized vascular density would be higher in lesional tissue compared to both control and perilesional samples due to processes related to epileptic activity and the underlying structural abnormality, and have no explanation for why we instead found no overall difference in vascular density between lesions and control samples, and lower perilesional vascular density.

Recent 7 tesla MRI studies showed an increased prominence of vascular structures colocalizing with cortical malformations in polymicrogyria ${ }^{122}$ and focal cortical dysplasia ${ }^{121}$. These vascular structures appeared to be mostly localized in the leptomeninges and sulci bordering the lesions. This was apparent on susceptibility-weighted contrast series, and therefore it could not be determined if this prominence was due to hemodynamic or structural changes, or a combination of both. Unfortunately, it was not possible to examine leptomeningeal vasculature in the current study, since meninges were not sufficiently preserved in the surgical specimens. 
Although in our study a significant increase vascular density in FCD lesions compared to normal controls could not be found, we did see an increase in vessel density in the FCD lesion compared to peri-lesional tissues.

Our findings do not provide conclusive support there are vascular structural changes that could be the substrate of possible identifying or localizing imaging features of FCD. Hemodynamic and consequent blood oxygen level variations possibly better explain vascular prominence on $T_{2}{ }^{*} \mathrm{MR}$ images.

ASL-MRI would have added valuable information regarding hemodynamic changes and possible correlation with vascular density and cytoarchitectural abnormalities, especially since prior studies showed conflicting results ${ }^{123-125}$. Unfortunately, ASL-MRI was not part of the standard pre-surgical diagnostic work-up and the retrospective nature of this study precluded collection of this data.

Sun et al. ${ }^{152}$ observed fine vascular structures in white matter of tubers on 7 tesla susceptibility weighted images. This imaging feature is compatible with the findings if our study, and potentially could be of use in characterizing tubers. It remains to be clarified if this conveys information on epileptogenicity of individual tubers.

\section{Conclusion}

Cortical vascular density decreases with age in healthy controls, while it increases with age and with epilepsy duration in patients with epilepsy due to FCD or tubers in TSC. White matter vascular density was not correlated with age or epilepsy duration. In dysplasia and tubers lesional brain tissue showed higher vascular density compared to perilesional tissue. Compared to controls, we observed an increase in microvasculature in white matter of TSC cortical tubers, which was linked to an inflammatory response.

The increased white matter vascular density of tubers compared to FCD suggests that it is the underlying pathology, rather than seizure activity itself, that explains differences in vascular index. The lesional cortical vascular density increase with age and duration of epilepsy, however, appears to suggest a direct effect of ongoing epileptic activity. 



\section{CORBELATION BETWEEN IN VIVO AND EX VIVO MULTIMODAL HIGH FIELD MRI AND HISTOPATHOLOGICAL PARAMETERS IN CORTICAL DYSPLASIAS}

Tim J. Veersema*, Sander J.H. Diederen*, Cyrille H. Ferrier Pieter van Eijsden, Jaco J.M. Zwanenburg, Herke Jan Noordmans Jeroen Hendrikse, Peter R. Luijten, Peter H. Gosselaar, Peter C. van Rijen Wim Van Hecke, Angelika Mühlebner, Kees P.J. Braun ${ }^{\circ}$, Eleonora Aronica ${ }^{\circ}$

* contributed equally to this manuscript $\ldots-\infty$ shared senior author

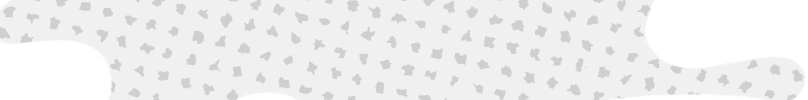

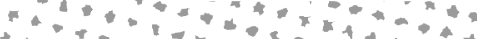

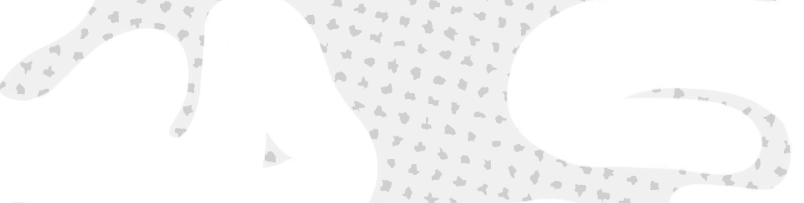




\section{Introduction}

In patients with refractory and focal lesional epilepsy, surgical resection can be considered. Presurgical evaluation of epilepsy has the main objective of defining the epileptogenic zone, including the location and extent of a brain structural abnormality ${ }^{153}$. MRI is an important diagnostic tool for identification of structural abnormalities. When cortical structural abnormalities are suspect current best clinical practice is to use epilepsy specific protocols at 3T that include 3D T1 and FLAIR sequences with isotropic resolution of $1 \mathrm{~mm}^{2}$, with assessment by dedicated neuroradiologist and incorporating electroclinical information. Post-processing techniques should be preferably used to support visual evaluation ${ }^{154}$. Unfortunately not all lesions are detected with current MRI imaging techniques, particularly so in patients suspected of minimal malformation of cortical development (mMCD) or with some subtypes of focal cortical dysplasia (FCD) ${ }^{42,70,92}$. In so-called MRI negative patients, ultra high-field MRI (7 tesla) can lead to an additional lesion detection in approximately a fifth of the patients ${ }^{99,155,156}$. Improving MRI lesion detection and delineation could lead to more refractory epilepsy patients becoming eligible for surgery and could improve surgical outcome.

The increased field strength of 7 tesla $(\mathrm{T})$ systems translates into improving spatial resolution and contrast in clinical practice ${ }^{157}$. The increased signal-to-noise ratio is generally not used to shorten the acquisition time. It remains unclear what is required to approach $100 \%$ detection, if ever feasible: should the focus be to improve spatial resolution or improve tissue contrast? Ideally, improved scanning techniques optimize specific tissue contrast based on histological changes in the targeted lesion type.

We initiated a comparative in vivo 7T MRI, ex vivo 7T MRI and histology project, in order to correlate histological characteristics of $\mathrm{mMCD} / \mathrm{FCD}$ lesions with specific MRI signal changes. With ex vivo 7T MRI, extremely lengthy scanning protocols are allowed, not restricted by Specific absorption rate (SAR) levels or affected by motion artefacts.

Previous quantitative neuroimaging and histology correlative studies have shown correlations between ex-vivo MRI signal changes and neuronal density, demyelinisation and balloon cells in FCD II ${ }^{158,159}$. In subtle dyslamination there is absence of the normal signal dip in cortical layer IV caused by the horizontal plexus and variation in cellularity and axonal density ${ }^{160}$. Analyses of ex-vivo 9.4T MRI revealed subtle white matter changes related to MMCD, while these lesions escape MRI detection in vivo ${ }^{159}$. These studies did not corporate in vivo MRI in the analyses. However, Goubran et al. ${ }^{161}$ and Zucca et al. ${ }^{162}$ have demonstrated successful co-registration of in vivo whole brain MRI and ex vivo MRI of surgical specimen.

Comparing in vivo with ex vivo MRI will demonstrate potential benefits of further increasing resolution and guide future technical developments. The eventual goal of this project is 
to improve the accuracy of MRI, both in increasing sensitivity for cortical dysplasia and in defining the borders with surrounding normal brain tissue. Moreover, better recognition of specific histological characteristics on MRI should aid in predicting histological MCD subtypes based on preoperative ultra high-field (UHF) MRI.

As a first step in this project, we report on the methodological framework that was constructed to allow analyses of image data of multiple modalities, which differ considerably in resolution and contrast, and which have to be reformatted into one space.

For optimal comparative analyses it is necessary to correct for deformations that occur at several different processing steps ${ }^{163}$. Deformations between in vivo and ex vivo MRI are caused by resection of the specimen and loss of coherence with connected structures, mechanical distortion and inhomogeneous shrinkage during fixation. Following the exvivo MRI the specimen is processed into histological slides during which stretching and shearing cause further deformation. As opposed to whole brain, co-registration of surgical resections poses an additional challenge since this requires part-to-whole registration ${ }^{161}$.

\section{Methods}

This is a prospective single centre cohort study, performed in line with the Declaration of Helsinki and Local Ethical committee approval was granted (protocol number 14-623/M). Prior to 7T MRI written informed consent was acquired from all patients or legal custodians. Surgical resection was performed exclusively for therapeutic reasons and the extent of excision was planned preoperatively and on the basis of the epileptogenic zone.

\section{Subjects}

Patients eligible for study inclusion were identified during regular multidisciplinary epilepsy surgery meetings. Patients were included if prior clinical 1.5 or 3T MRI had been performed using epilepsy dedicated scanning protocols and mMCD/FCD was suspected as underlying cause, with or without the presence of another, possibly associated primary lesion (such as mesiotemporal sclerosis, LEAT, vascular malformation or traumatic brain injury at young age, all potentially related to FCD subtype III). Patients were also eligible when standard MRI did not reveal clear abnormalities; so-called "MRI-negative" patients.

Excluded were patients with contra-indications for 7T MRI, those who were unable to undergo unsedated imaging and all children under the age of six years.

Study participants underwent additional 7T MRI imaging during their pre-surgical work-up. Patients were also invited for the study if 7T MRI had already been requested by the treating physician in the context of a suspected developmental abnormality but with normal 1.5 or 3T MRI. These "clinical" 7T scans were acquired using the same scanning protocol and the 
work-flow after surgery (ex vivo MRI and histopathology) was identical to that described below.

\section{MRI Acquisition}

\section{In vivo 7T MRI}

All in vivo scans were performed on a whole-body 7 tesla MRI system (Philips Healthcare, best, Netherlands) with a volume transmit coil and 32-channel receive head coil (Nova Medical, Wilmington, Massachusetts, USA). Dielectric pads, containing calcium titanate (Leiden University Medical Center, Leiden, the Netherlands), were placed bilateral to the head to reduce artefacts and signal loss in the temporal regions ${ }^{101}$.

The 7T scanning protocol consists of $T_{1^{\prime}} T_{2^{\prime}}$ FLAIR, and $T_{2}{ }^{*}$-weighted sequences, and a gradient echo white matter suppression (WMS) sequence, all with $0.5-0.8 \mathrm{~mm}$ acquired resolution; acquisition time was circa 45 minutes. All sequences are routinely reformatted in $0.5 \mathrm{~mm}$ (interpolated) multiplanar reconstructions to have axial, coronal and sagittal images for all sequences, allowing for optimal appreciation of cortical architecture. This protocol covers all sequences recommended in various guidelines for the detection of epileptogenic lesions ${ }^{100}$. Parameters of the in vivo 7T MRI sequences performed in our centre are described in Table 1.

\section{Ex vivo $7 T M R I$}

Tissue samples were scanned using the same hardware as the in vivo 7T MRI acquisition. Tissue fixation with formalin reduces tissue $T_{1}$ and $T_{2}$ relaxation times ${ }^{164}$, and accordingly scanning parameters were chosen to produce images that resemble tissue contrast in vivo. Long acquisition times and less stringent SAR limitations allowed 0.18-0.40 mm isotropic resolution in combination with high tissue contrast. Image based B0 shimming was performed, and manual power optimization was used to obtain correct flip angles. Homogeneity correction based on pre-scan sensitivity maps was applied to all sequences except WMS. No SENSE acceleration was used. The used sequences and main parameters are provided in table 2 .

While the $T_{1}, T_{2}, T_{2}$ * and WMS sequences yielded consistent high quality images, the FLAIR sequence was of variable and often mediocre quality, even after increasing the $\mathrm{TI}$ and TR from an initial $1150 \mathrm{~ms}$ and $5000 \mathrm{~ms}$ to 1600 and $8000 \mathrm{~ms}$, respectively, and thereby drastically increasing acquisition time.

Directly after scanning the ex vivo MRI was visually assessed and the optimal orientation for image reconstruction was chosen to obtain orthogonal cutting angles through the cortical layers and underlying white matter, needed for accurate evaluation of histomorphological abnormalities. Multiplanar reconstructions (MPR) were made with $0.2 \mathrm{~mm}$ slice thickness. 
Table 1. In vivo and ex vivo 7TMRI protocol and parameters.

\begin{tabular}{|c|c|c|c|c|c|c|c|c|c|}
\hline & & $\begin{array}{l}\text { TE } \\
\text { ms }\end{array}$ & TI ms & TR ms & $\begin{array}{l}\text { FA } \\
\text { degrees }\end{array}$ & $\begin{array}{l}\text { Acquired } \\
\text { resolution } \\
\text { mm }\end{array}$ & $\begin{array}{l}\text { Matrix (AP x } \\
\text { RL) x slices }\end{array}$ & $\begin{array}{l}\text { SEN- } \\
\text { SE R }\end{array}$ & $\begin{array}{l}\text { Acquisition } \\
\text { time } \\
\text { min:sec }\end{array}$ \\
\hline \multirow[t]{5}{*}{$\begin{array}{l}\text { in } \\
\text { vivo }\end{array}$} & $\begin{array}{l}\text { 3D } T_{1} \mathrm{w} \\
\text { TFE }\end{array}$ & 2.9 & 1200 & $9 / 3500^{b}$ & 6 & $0.81 / 0.81 / 0.80$ & $\begin{array}{l}248 \times 312 x \\
475\end{array}$ & 2.6 & $9: 34$ \\
\hline & $\begin{array}{l}\text { 3D } T_{2} \mathrm{w} \\
\mathrm{TSE}\end{array}$ & 302 & - & 3200 & 90 & 0.70/0.70/0.70 & $\begin{array}{l}356 \times 357 x \\
543\end{array}$ & 5.6 & $10: 24$ \\
\hline & $3 \mathrm{D} T_{2}{ }^{*} \mathrm{w}$ & 27 & - & 88 & 24 & $0.50 / 0.50 / 0.50$ & $\begin{array}{l}480 \times 381 \times \\
600\end{array}$ & 2.3 & $7: 23$ \\
\hline & $\begin{array}{l}\text { 3D MP- } \\
\text { FLAIR }\end{array}$ & 300 & 2200 & 8000 & 90 & $0.80 / 0.82 / 1.00$ & $\begin{array}{l}312 \times 304 x \\
380\end{array}$ & 7.5 & $8: 40$ \\
\hline & 3D WMS & 2.0 & 600 & $6.73 / 10000^{c}$ & 4.5 & $0.80 / 0.80 / 0.80$ & $\begin{array}{l}320 \times 320 x \\
474\end{array}$ & 4 & $8: 42$ \\
\hline \multirow[t]{6}{*}{$\begin{array}{l}\text { ex } \\
\text { vivo }\end{array}$} & 3D $T_{1}$ FFE & 3.6 & 700 & $7.8 / 2000^{d}$ & 6 & $0.40 / 0.40 / 0.40$ & $\begin{array}{l}348 \times 384 x \\
100\end{array}$ & 1 & 1:09:37 \\
\hline & 3D $T_{2}$ TSE & 163 & - & 2000 & 90 & $0.40 / 0.40 / 0.40$ & $\begin{array}{l}500 \times 210 x \\
221\end{array}$ & 1 & 0:44:30 \\
\hline & $\begin{array}{l}\text { 3D } T_{2}{ }^{*} \mathrm{w} \\
\text { FFE } 0.22 \mathrm{~m} \\
\text { a }\end{array}$ & 30 & - & 75 & 30 & $0.22 / 0.22 / 0.22$ & $\begin{array}{l}680 \times 365 x \\
181\end{array}$ & 1 & $1: 37: 11$ \\
\hline & $\begin{array}{l}3 \mathrm{DD} T_{2}{ }^{*} \mathrm{~W} \\
\mathrm{FFE} \\
0.18 \mathrm{~mm}\end{array}$ & 25 & & 75 & 35 & $0.18 / 0.18 / 0.18$ & $\begin{array}{l}823 \times 445 x \\
222\end{array}$ & 1 & $1: 36: 43$ \\
\hline & 3D FLAIR & 164 & 1600 & 8000 & 90 & $0.40 / 0.40 / 0.40$ & $\begin{array}{l}500 \times 210 x \\
221\end{array}$ & 1 & $3: 31: 20$ \\
\hline & $\begin{array}{l}\text { 3D WMS } \\
\text { FFE }\end{array}$ & 4.3 & 300 & $9.2 / 3000^{\mathrm{e}}$ & 6 & $0.40 / 0.40 / 0.40$ & $\begin{array}{l}348 \times 199 x \\
111\end{array}$ & 1 & 2:02:33 \\
\hline
\end{tabular}

$\mathrm{TE}=$ echo time; $\mathrm{TI}=$ inversion time; $\mathrm{TR}=$ repletion time; $\mathrm{FA}=$ flip angle; SENSE R = SENSitivity Encoding acceleration factor;

TFE = Turbo Field Echo (gradient echo); TSE = Turbo Spin Echo; $T_{2}{ }^{*} \mathrm{~W}=T_{2}{ }^{*}$ weighted (susceptibility weighted contrast); FFE = Fast Field Echo (gradient echo); MP-FLAIR = magnetization prepared - Fluid-attenuated Inversion Recovery ${ }^{108}$; WMS = White Matter Suppression sequence (gradient echo); SE = Spin Echo; SPAIR = Spectral selection Attenuated Inversion Recovery (fat suppression) $\mathrm{DW}=$ Diffusion Weighted; gado = gadolinium intravenous contrast; dual = dual echo; $\mathrm{SSH}=$ Single-Shot.

a $3 \mathrm{D} T_{2}{ }^{*}$ W FFE $0.22 \mathrm{~m}$ uses 1.5 factor oversampling for improved SNR.

${ }^{\mathrm{b}}$ TR between excitation and inversion pulses respectively, TFE factor 245.

'TR between excitation and inversion pulses respectively, TFE factor 233.

${ }^{\mathrm{d}}$ TR between excitation and inversion pulses respectively, TFE factor 60 .

e TR between excitation and inversion pulses respectively, TFE factor 36 . 


\section{Sample preparation for ex-vivo 7T MRI and immunohistochemistry}

Tissue samples of 17 patients were of sufficient size for further analyses and were processed according to the study protocol. One author (TV) was present during each surgical procedure to observe and register orientation of the resected specimen. Neurosurgeons in our centre strive for en-bloc resection of the lesion to aid histopathological examination. When needed, colour markings were applied. The fresh tissue was directly transported to the pathology lab and immersed in $4 \%$ buffered formaldehyde for at least 48 hours, considering a diffusion rate of $1 \mathrm{~mm}$ per hour within the tissues. Complete fixation is essential for homogeneous MRI signal throughout the specimen ${ }^{160}$ and for tissue preservation for histopathological diagnosis. The fully fixated specimen was then embedded in Agar (5\%) in a rectangular mould. After cooling, the block is stable and can be handled safely. One corner of the agar block was marked in the $X, Y$ and $Z$ axes for later spatial reference. Barrel cactus needles were used for these purposes, which proved optimal due to their rigidity and lack of magnetic properties and susceptibility effects. The tissue and agar block was fixated in a plastic tray and submersed in Perfluoropolyether fluorinated lubricant, a highly stable protein-free substance with no MRI signal, (Fomblin ${ }^{\circledR}$, Solvay) to further reduce imaging artefacts. The tray was then placed in the head coil for scanning. Using a spinning blade slicing machine, $4 \mathrm{~mm}$ slabs were cut from the Agar-tissue block. The sectioning plane was identical to the ex-vivo multiplanar reconstructions.

Resected tissue was included integrally in histology cassettes. Tissue slices were processed into liquid paraffin and sectioned at $5 \mu \mathrm{m}$ with a microtome (Leica Biosystems, Nußloch, Germany), and mounted on positively charged slides. All tissue samples were histologically examined using hematoxylin \& eosin (HE) and NeuN (NEUronal Nuclei). These examinations were essential for clinical pathological diagnosis. Based on these slides, for more extensive immunohistological characterization, 2-3 tissues blocks were selected that provided optimal tangential sectioning of the dysplastic lesion. $\mathrm{HE}$ and $\mathrm{NeuN}$ stainings were complemented with eight immunohistological stainings commonly used to diagnose and further specify focal developmental abnormalities in epilepsy patients (MAP-2, Vimentin, SMI32, SMI31, CD34, GFAP, MBP, Olig2).

For the detection of the inflammatory cells and proinflammatory pathways the following antibodies were used: NeuN (1:100, clone A60, Chemicon, Billerica, MA, USA) stains neuronal nuclei; MAP-2 (microtubule associated protein 2; 1:500 polyclonal rabbit, AB5622, Merck, Amsterdam, the Netherlands) a neuronal marker preferentially labelling dendrites. Vimentin (1:500, clone V9, Dako, Glostrup, Denmark) stains type III intermediate filament (IF) protein and is a marker for balloon cells, the hallmark feature of type Ilb dysplasia; SMI32 antibody (non-phosphorylated neurofilament $\mathrm{H}, 1: 1000$ dilution, clone SMI32, Sternberger, Lutherville) is a marker for immature or dysmorphic neurons as present in FCD type lla; SM31 antibody (neurofilament H 1:1000, clone SMI31, Sternberger, Lutherville) stains axons. CD34 (cluster 
of differentiation 34, 1:600, Qbend, Immunotech) used as used as a marker of vascular endothelial cells to determine vascular density; GFAP (glial fibrillary acid protein, 1:4000, Dako, Glostrup, Denmark) as astrocytic marker; MBP (myelin binding protein, 1:400 DAKO) as major myelin constituent; Olig2 (Oligodendrocyte lineage transcription factor 2, 1:100 dilution, IBL, Minneapolis, USA) as oligodendroglial lineage marker.

All immunohistochemical stainings were performed with a Ventana semi-automated staining machine (Benchmark ULTRA; Ventana, Illkirch, France) and the Ventana DAB staining system according to the manufacturer's protocol. Slides were air-dried overnight at $37^{\circ} \mathrm{C}$. Histopathological classification was performed in accordance with the $2011 \mathrm{ILAE}$ guidelines for $\mathrm{mMCD}$ and $\mathrm{FCD}^{13}$. Histological slides were digitalized at $10 \mathrm{x}$ magnification at $648,58 \mathrm{~nm} /$ pixel resolution (Olympus life sciences, Waltham, Massachusetts, USA) and saved in BigTiff format for further analyses.

ROI's for lesional and perilesional areas in grey and white matter were drawn based on visual inspection of MAP-2 stained digital histology slices. Tissue was considered white matter if $>500 \mu \mathrm{m}$ away from the grey-white matter border. Perilesional cortex was only available in FCD type II lesions and defined by the absence of cortical dyslamination, dysmorphic neurons and balloon cells.

To develop the methodological pipeline of this multimodality study, a subset of cases were selected first. In order to achieve optimal co-registration, these were patients with the largest resected tissue samples, and in whom ex vivo MRI artefacts were minimal.

\section{Co-registration}

\section{Histology}

Firstly, because of its high resolution, all histological sample image files were resized to enable co-registration and histological analysis, resulting in a spatial resolution of $25 \mathrm{DPI}$. Different histological samples were aligned using an in-house developed software tool ${ }^{165}$. MAP-2 images were used as a reference to which all other stainings were registered. All stainings were aligned interactively after which an affine transformation algorithm (including translation, rotation, scaling and sheering) was performed. Results were checked manually and a non-rigid registration was performed subsequently using Elastix (Image Sciences Institute, UMC Utrecht, Utrecht, the Netherlands) (see diagram 1).

\section{Histology to ex vivo 7T MRI}

Histological samples were sectioned in the same orthogonal angle as the optimal ex vivo 7T MRI multiplanar reconstruction (as described above). Since histological spatial resolution was much higher, ex vivo 7T MRI sequences were rescaled in the $X$ and $Y$ direction matching histological slides. Subsequently these $T_{1}, T_{2}^{*}$ and WMS sequences were registered to $T_{2}$ sequences using affine and non-rigid (Elastix) registration algorithms. MRI slices corresponding 


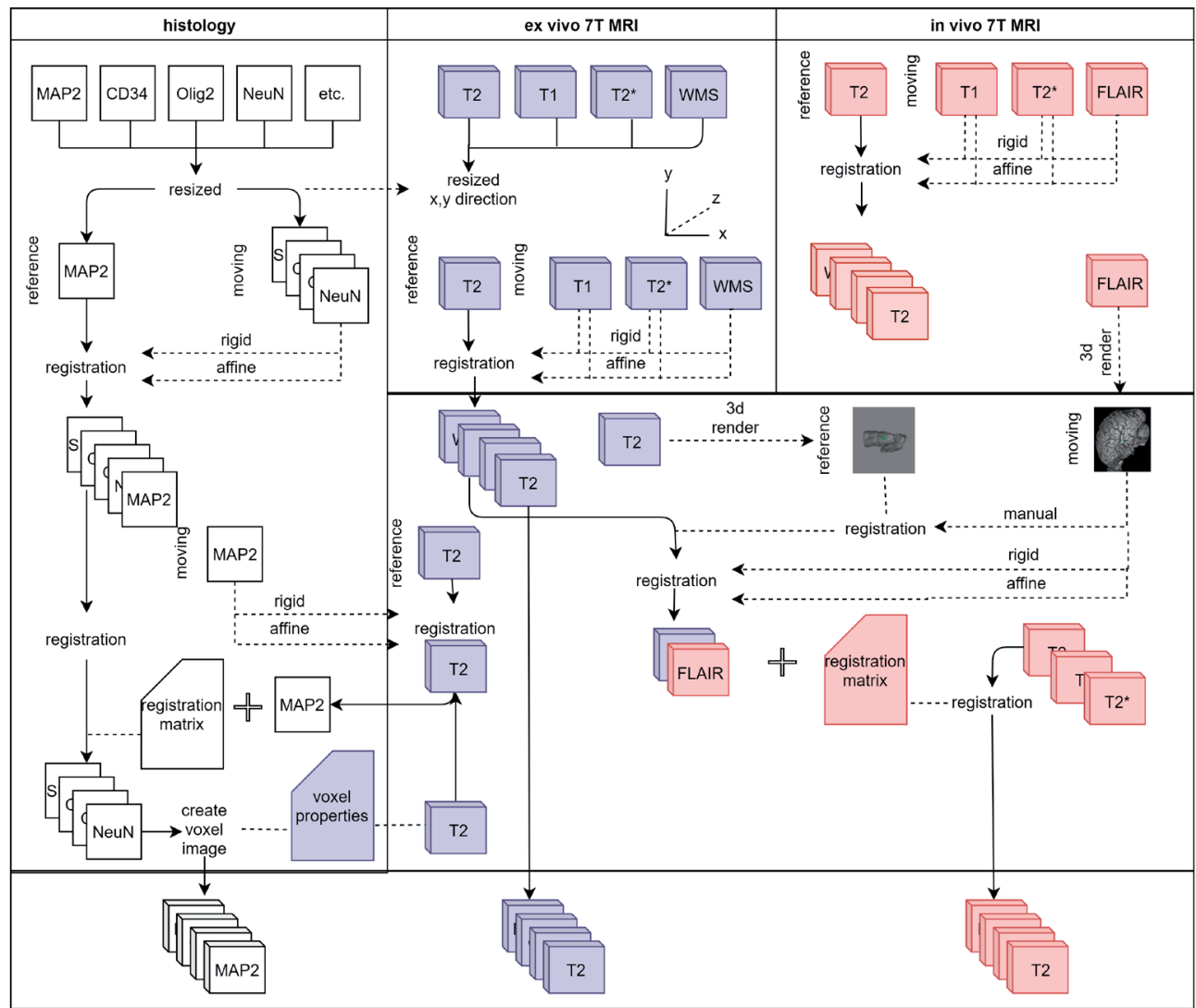

Diagram 1: Diagram showing different registration steps.

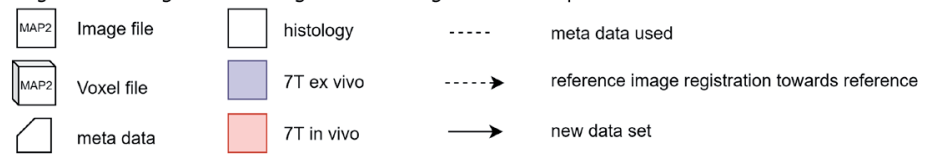

to histological slides were manually allocated. The allocated $T_{2}$ slices were used as a reference in the next registration step. MAP-2 shares the clear differentiation between white and grey matter with its radiological counterpart, the T2 sequence. Therefore, these slides were registered using the same steps as described above in co-registration histology section. The obtained transformation matrix is applied to the spatially corresponding slides (see diagram 1).

\section{Ex vivo $7 T M R I$ to in vivo $7 T M R I$}

All in vivo $7 \mathrm{~T} M \mathrm{MRI}$ scans were registered to the $T_{2}$ sequence using an affine and non-rigid (Elastix) registration algorithm. Subsequently, in-house developed software (java navigation, described previously by Noordmans et al. ${ }^{166}$ ) was used to construct a 3D rendering of the cortex using the registered Flair in vivo sequence and of the resected tissue using the $T_{2}$ ex 
vivo sequence. Photographs obtained during surgery and orientation markers were used to find the resected area on the in vivo scan. Cortical landmarks were placed on both in vivo and ex vivo renderings. These were used to manually overlay the ex vivo Flair image over the $T_{2}$ ex vivo image. Results were checked in all three planes. Subsequently the ex vivo $T_{2}$ image was used as a reference and the FLAIR image is registered using both affine and non-rigid transformation. The obtained transformation matrix was applied to all other in vivo sequences and the in vivo scans are resliced in the same orientation as the ex vivo images (see diagram 1).

\section{Histology to in vivo 7T MRI}

Since the histological images were already registered to the corresponding slices in the ex vivo images, we were able to construct a voxel image in which on the Z-axis histology images were put corresponding to the allocated slices in the ex vivo image. All images in the Z-direction without a corresponding histology image remained empty. The histological voxel images correspond to the registered and resliced in vivo 7T MRI images (see diagram 1).

\section{ROI selection}

Whole slide images (WSI) are used to define the type of lesion according to the most recent ILAE ${ }^{13}$ and $\mathrm{WHO}^{167}$ classifications. Subsequently, lesion centre, lesion border and normal tissue regions were manually defined based on MAP2 immunohistochemistry and HE staining. All ROI were delineated in FIJI (ImageJ2). In the corresponding slices of both 7T in vivo and ex vivo imaging, the tissue visible in histology samples was also defined as centre, border, and normal tissue, and manually delineated as such.

\section{Results}

33 subjects underwent in vivo 7T MRI, of which 21 on clinical indication (i.e. suspected mMCD/ FCD but normal previous imaging on lower-field MRI) and 12 for research purposes only, in whom previous imaging at lower-field had already revealed a lesion. In one patient 7T MRI failed due to patient non-compliance. In 7 of these 33 patients there was no indication for resective surgery. Five underwent surgery outside the study period, precluding ex vivo MRI analyses. Twenty-one patients underwent resective epilepsy surgery in the study period. The resected specimen was not suited for ex vivo imaging in four of those. In 17 subjects sufficient tissue was resected to allow ex vivo MRI imaging (figure 1). Frequency of ILAE histopathological diagnoses was: $m M C D$ type $2 n=8$; FCD Ila $n=1$; FCD Ilb $n=5 ; F C D$ IId $n=1$, Gangliogloma $n=1$; glioneuronal tumour-not otherwise specified $=1$. Seven tissue samples were selected for preliminary analyses; $m M C D n=3$; FCD Ila $n=1$; FCD Ilb $n=3$. 

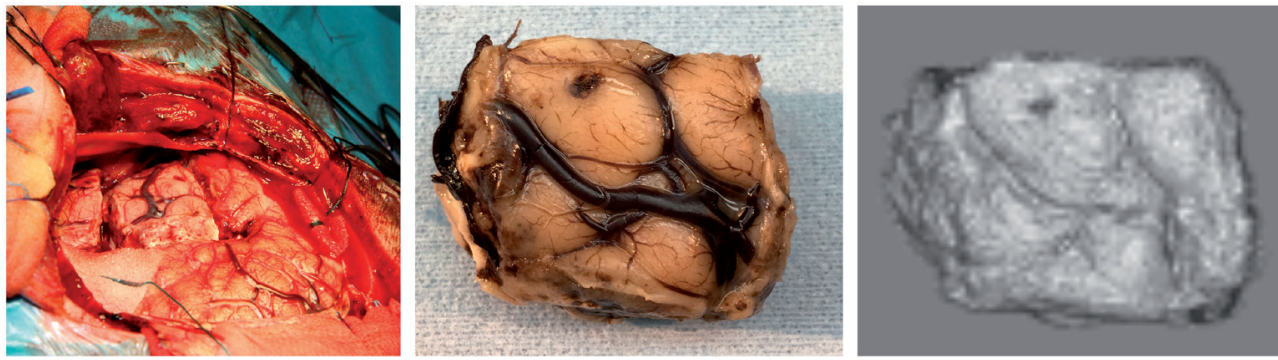

Figure 1. Example of resected brain tissue (patient number S03).

Left resected area visible during surgery; Middle resected tissue; Right 3D render of $7 \mathrm{~T}$ ex vivo MRI image of resected tissue.

Clinical information of the seven patients that were selected for this preliminary study to construct and optimize the methodological pipeline for the proposed multimodality study, as described above, is supplied in table 2 .

In each of the seven resected brain tissue samples, two or three sections were used to obtain 10 different stainings. These were all registered towards the MAP-2 slides. Corresponding ex vivo MRI images were selected and histology samples were registered towards ex vivo imaging (see figure 2).

$3 \mathrm{D}$ renders of both in vivo and ex vivo 7T MRI images were constructed. These were used together with photographs obtained during surgery to manually align the seven resected brain samples to the in vivo 7T imaging, after which an affine en non-rigid registration was performed, resulting in registered $7 \mathrm{~T}$ in vivo and ex vivo images (see figure 3 ).

Voxel information of the 7T ex vivo scans was used to create a voxel image of all stainings. These combined registration steps resulted in registered image data of all 10 stainings, 7T ex vivo $T_{1}, T_{2}$ and WMS MRI imaging and 7 T in-vivo $T_{1}, T_{2^{\prime}} T_{2}{ }^{*}$ and FLAIR imaging (example of co-registered ex vivo T2 MRI and MAP2 staining is given in figure 4).

ROI selection was performed on $7 \mathrm{~T}$ in vivo MRI imaging, $7 \mathrm{~T}$ ex vivo MRI imaging and histological samples. Lesion centre, lesion border and normal tissue regions were delineated manually.

\section{Discussion}

In the comparative analysis of different imaging modalities accurate registration is key. Although challenging, registration of histological stainings, ex vivo acquired UHF imaging of resected brain tissue and pre-operative acquired UHF brain imaging can be performed. 


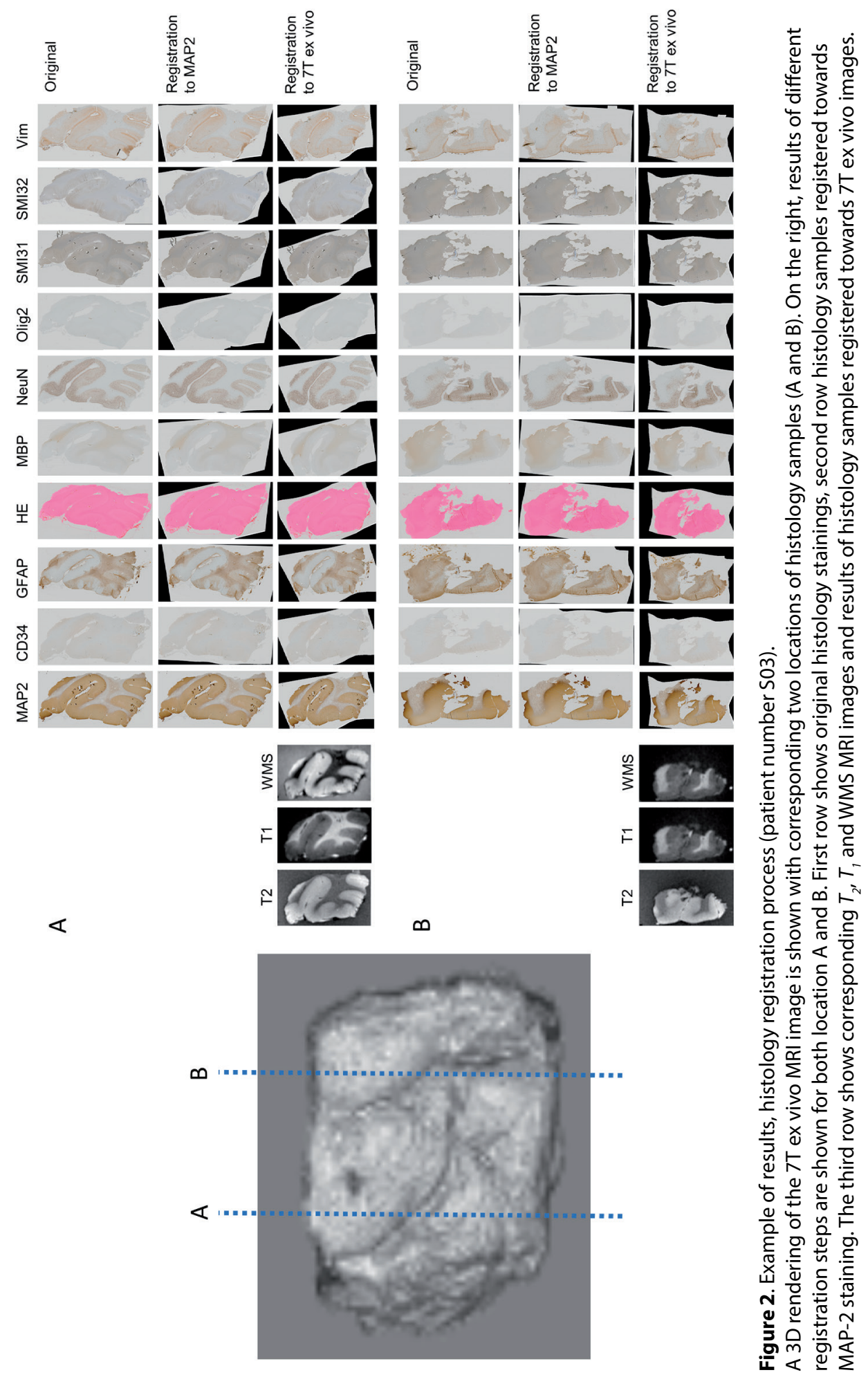




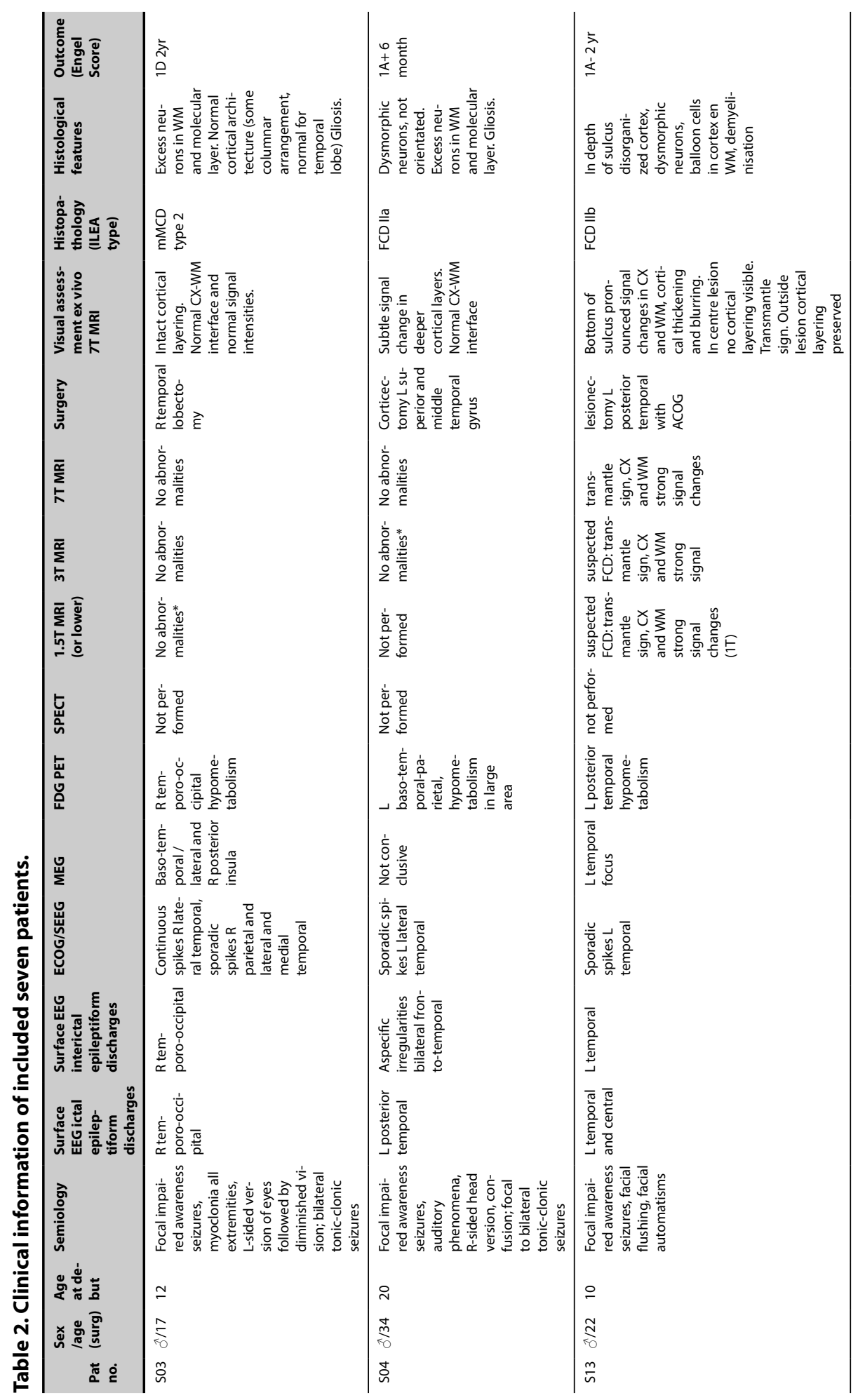




\begin{tabular}{|c|c|c|c|c|}
\hline 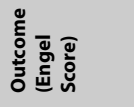 & $\begin{array}{l}\grave{2} \\
\frac{1}{5}\end{array}$ & 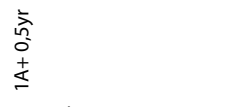 & 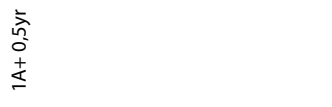 & 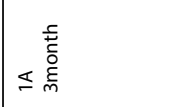 \\
\hline 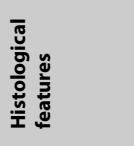 & 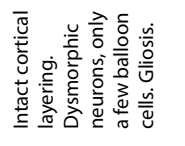 & 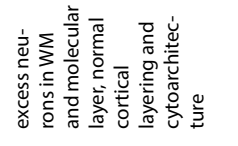 & 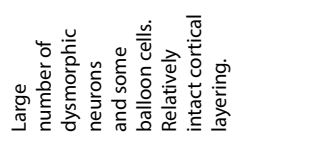 & 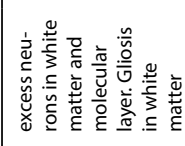 \\
\hline 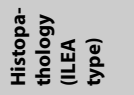 & $\begin{array}{l}\stackrel{0}{\bar{O}} \\
\text { Uે }\end{array}$ & 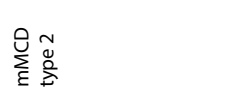 & 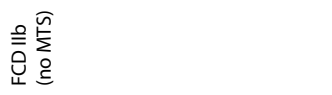 & 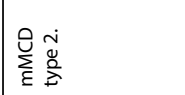 \\
\hline 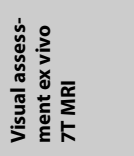 & 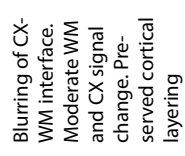 & 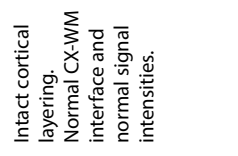 & 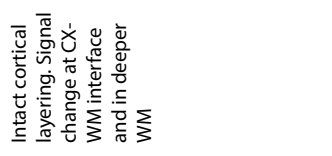 & 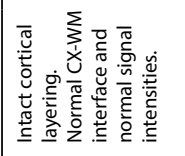 \\
\hline 空 & 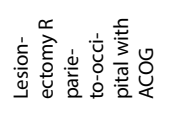 & 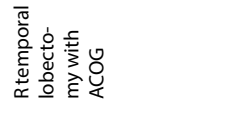 & 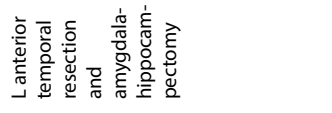 & 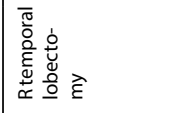 \\
\hline & 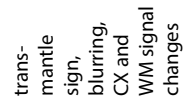 & 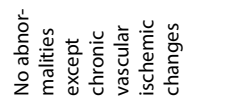 & 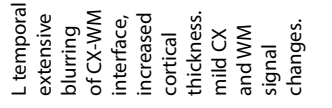 & 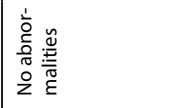 \\
\hline & 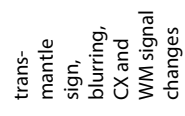 & 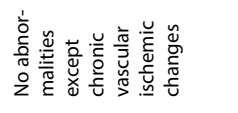 & 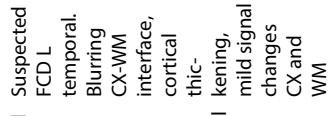 & 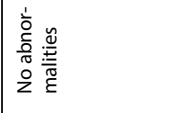 \\
\hline 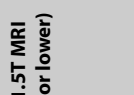 & 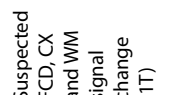 & 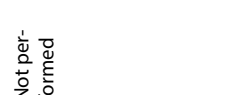 & 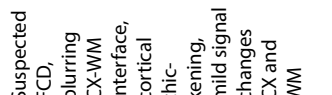 & 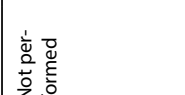 \\
\hline tu & 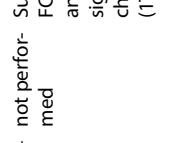 & 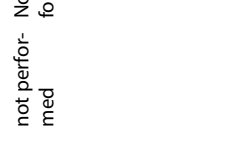 & 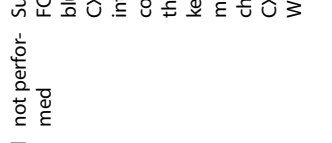 & 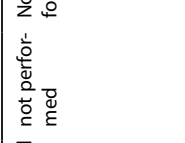 \\
\hline & 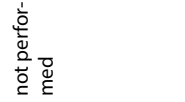 & 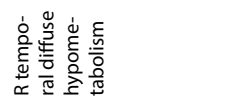 & 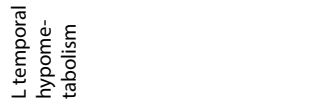 & 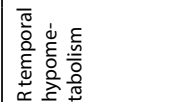 \\
\hline 迆 & 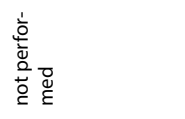 & 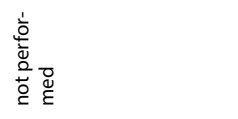 & 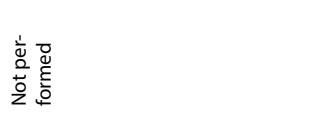 & 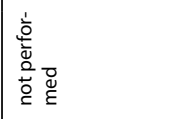 \\
\hline 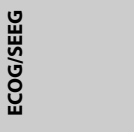 & 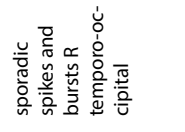 & 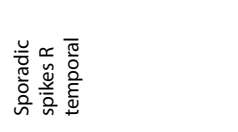 & 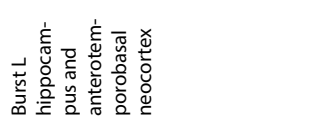 & 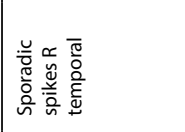 \\
\hline 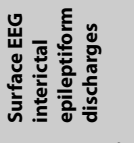 & 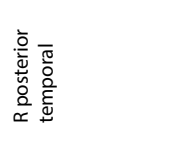 & 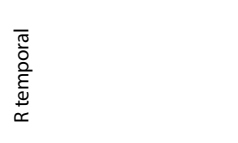 & 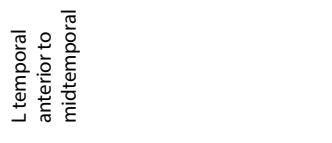 & 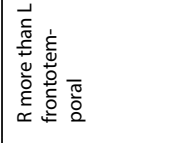 \\
\hline 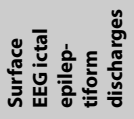 & 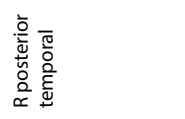 & 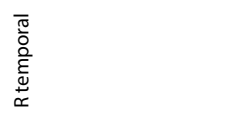 & 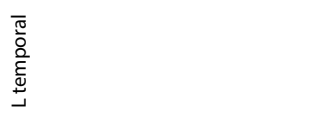 & 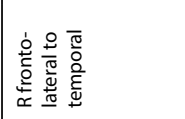 \\
\hline वे & 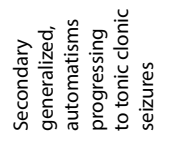 & 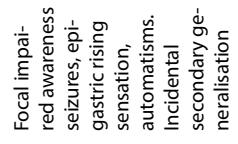 & 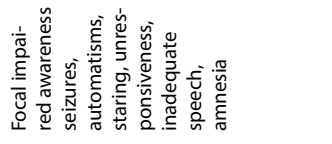 & 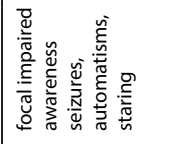 \\
\hline 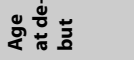 & $\underline{m}$ & \pm & $\stackrel{\circ}{\circ}$ & $\stackrel{\Omega}{ }$ \\
\hline × ฐ 홈 & $\stackrel{0}{\frac{2}{0}}$ & 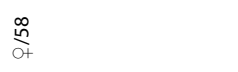 & $\frac{\bar{o}}{0+}$ & 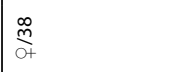 \\
\hline 芯 & $\hat{n}$ & $\stackrel{\sim}{\sim}$ & $\stackrel{\sim}{\sim}$ & $\tilde{n}$ \\
\hline
\end{tabular}



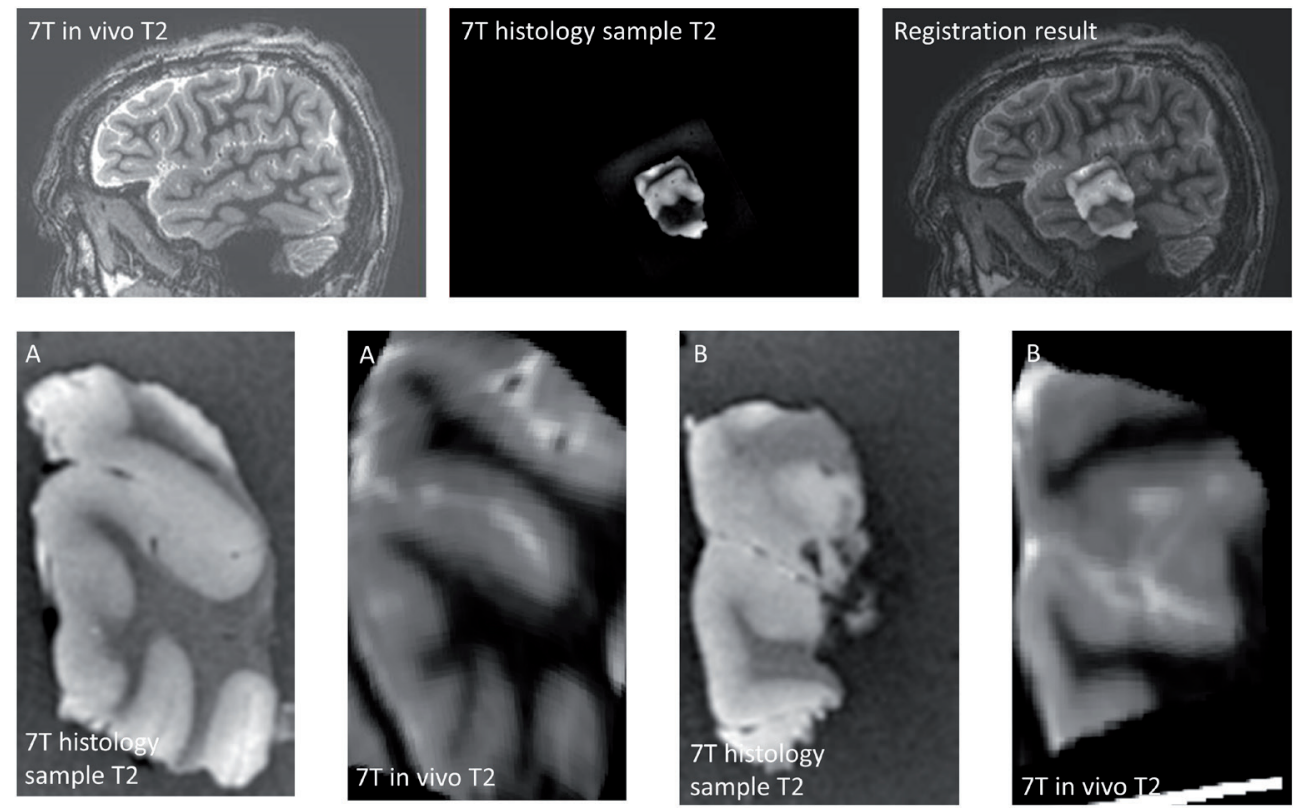

Figure 3. Example of registration result of in vivo 7T and ex vivo 7T imaging for histology location $A$ and $B$ (patient number S03).

However, extensive documentation during surgery, meticulous tissue preparation and scanning is necessary to obtain samples usable for further analysis. Moreover, sample size needs to be of sufficient in order to register accurately.

The described registration pipeline currently requires numerous registration steps, making registration time consuming. In-house developed software was used and further development of this software is needed to allow for more automated registration. This will result in the possibility to register bigger volumes of data. Moreover, the vast amount of data requires meticulous data handling. The development of a data management system could greatly reduce time and human errors.

In our ongoing research, we will assess differences of lesion type and allocated area of lesion centre, lesion border and normal tissue between histology samples, in vivo MRI and ex vivo MRI imaging. Furthermore, we will perform line profile analyses and histogram comparison to quantitatively correlate MRI and histology, resulting in a novel comparison between 7T in vivo $M R I$ imaging and histology samples. 

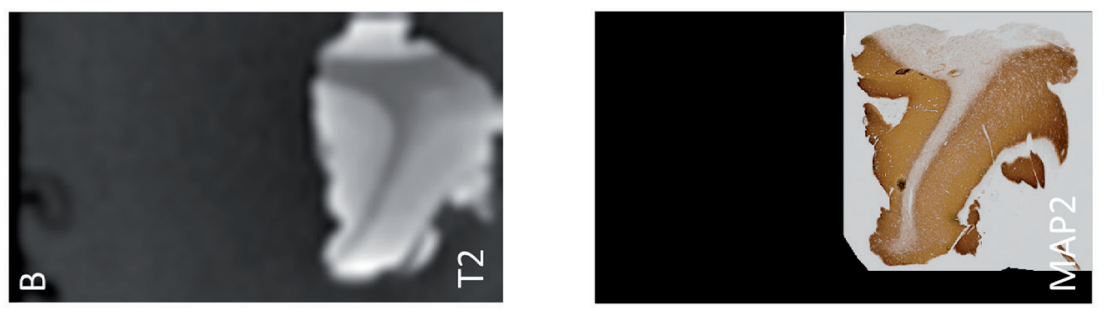

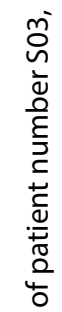
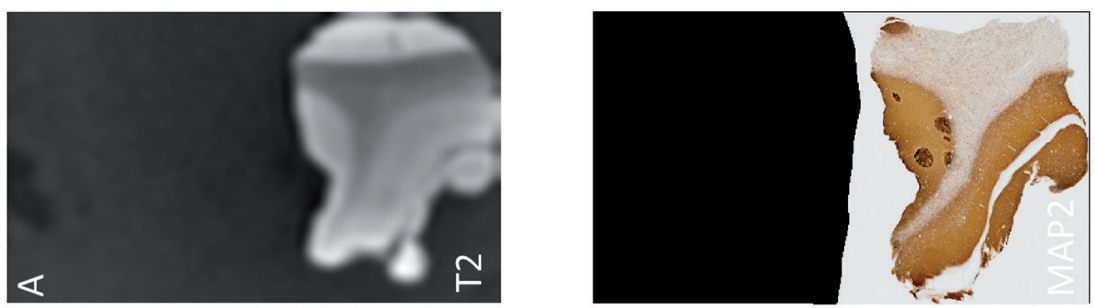

崩
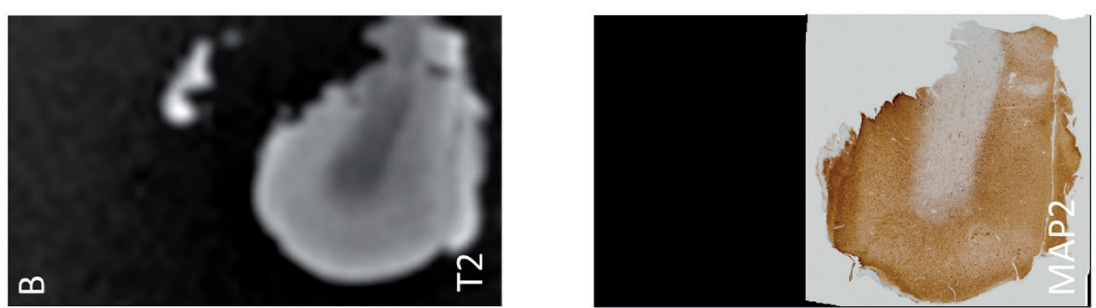

ثै

웡

용

\&

凹 엉

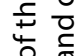

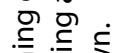
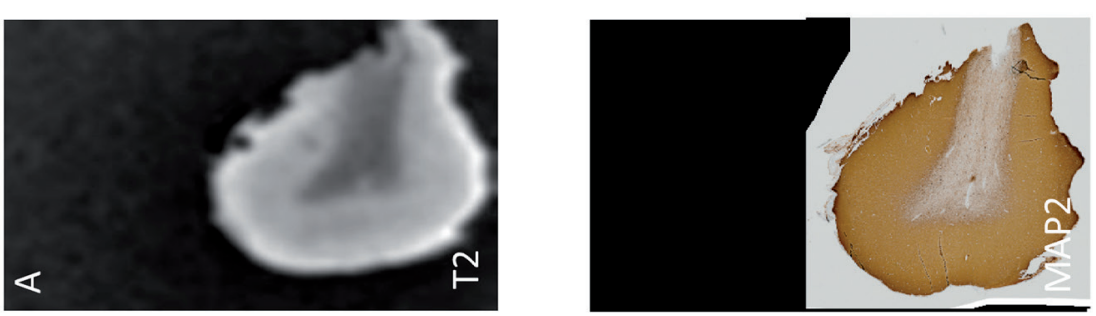

둥

जै है

กิ

$\sum^{1} \rho$

$>$ ज

$\frac{1}{2} \times \frac{1}{2}$

뉸

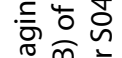

हิ ฮิ ซ

$\stackrel{\circ}{\circ}$ ह
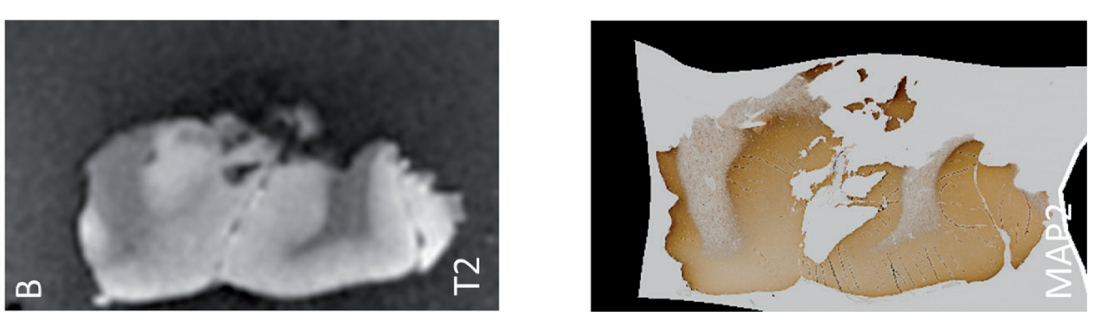

$0 \leqslant 5$

$\gg 气$

뜬 음

4 즁
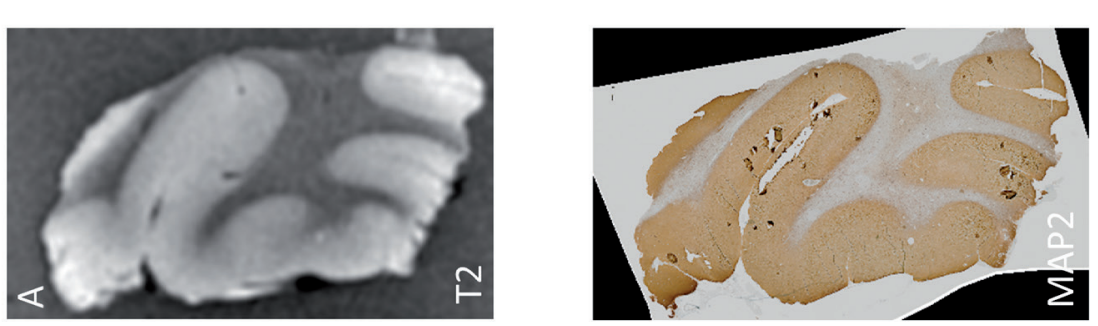

$\pm \frac{1}{5}$ 응 눙

ญ ฏ

ᄃㄴㄷㅇ

은

言 气

क्षे

능 은

次

로

可 긍

× $\circ$ व

o 引

$\pm$

은 


\section{Conclusion}

This study demonstrates the feasibility to register histological samples of resected brain tissue in epilepsy patients with both ex vivo UHF MRI imaging of resected brain tissue and pre-surgical (in vivo) UHF MRI imaging. We show a pipeline in which all different steps are described and display registration results. Registration of these modalities makes it possible to perform further comparative analysis. Furthermore, comparison of histology and MRI imaging data can provide new insights in the development of novel post-processing techniques, with potential to improve the detection of subtle cortical dyslamination in vivo. This could both improve accuracy of presurgical planning and hence seizure outcome. 



\section{Summary}

Patients with refractory epilepsy who undergo surgical treatment frequently have mMCD of FCD as the underlying cause. In this thesis we aimed to expand understanding of how histological differences (as defined in the most recent ILAE classification) relate to patient characteristics and successful surgical therapy. Moreover, we explored ways to improve diagnostic imaging for subtle lesions that escape detection by current techniques fail to identify. More accurate identification of the presence and delineation of MMCD and FCD lesions will eventually lead to more patients benefitting from surgical therapy and improving surgical outcome.

The first step was to evaluate the histopathological diagnoses and clinical characteristics in patients operated before. We retrospectively studied a consecutive cohort of all patients who underwent epilepsy surgery in the UMC Utrecht over a period of 12 years (2000-2012) with a histopathological of mMCD or FCD according to the 2011 ILAE guidelines. In the past decades there has been considerable inconsistency in the used definitions for these specific entities. The 2011 guidelines are the most recent attempt to harmonize histopathological classification. For the research described in this thesis we revised the histopathological diagnosis in the majority of tissue samples in accordance with the new classification.

In chapter $\mathbf{2}$ we described this cohort of 88 patients of whom during surgery tissues had been obtained for histology and an unequivocal diagnosis could be made. We found a strikingly high number of patients with $\mathrm{mMCD}(31 / 88,35 \%)$ in our cohort, while FCD I made up $9 \%(8 / 88)$ and FCD II 56\% (49/88) of the study population. Considerably less patients were diagnosed with $\mathrm{mMCD}$ in previous studies, and more with FCD I ${ }^{10}$. We observed a predilection of FCD II types for the frontal lobe, while mMCD and FCD I were most often localised in the temporal lobes. Patients with mMCD had onset of epilepsy later in life and consequently underwent surgery at older age compared to those with FCD.

Epilepsy surgery resulted in lasting seizure freedom rate of $49 \%$ at a median follow-up of 7 years; $36 \%$ had also discontinued anti-epileptic medication. Patients with mMCD were less likely to reach seizure freedom. Patients with FCD Ilb had a markedly increased chance of achieving seizure freedom.

In a subset of this cohort (42 children), we studied cognitive ability prior to, and after surgery. These findings are described in Chapter 3. Among the 42 children who underwent cognitive testing, $51 \%$ had intellectual disability prior to surgery. Preoperative cognitive functioning was lower in patients with onset at younger age and patients with severe delay $(\mathrm{IQ} / \mathrm{DQ}<55)$ were more often operated at very young age, leading to a spurious correlation low cognitive function, and surgery at young age and short epilepsy duration. In patients with IQ/DQ $>55$, shorter epilepsy duration was associated with better cognitive functioning. 
All FCD I patients had a cognitive disability and their mean IQ/DQ was significantly lower than that in children with mMCD or FCD II.

Following surgery, relevant improvement, defined as $\geq 7$ IQ/DQ points, was seen in $43 \%$ of the children, while cognitive functioning was stable in $46 \%$ and deteriorated in $11 \%$. Post-surgical cognitive functioning was strongly related to pre-surgical functioning. Only a diagnosis of epileptic encephalopathy predicted cognitive improvement after surgery. We did not observe an association between postoperative cognitive outcome and seizure status.

In Chapter 4 we studied a series of 40 patients who underwent additional 7 tesla MRI in the presurgical diagnostic trajectory. 7 tesla MRI was indicated for patients with refractory and presumed lesional focal epilepsy, and the absence of a lesion on one or more previous lower field strength of sufficient quality, or when concealed pathology was suspected in combination with MR-visible hippocampal sclerosis, so-called dual pathology. Multidisciplinary evaluation of 7 tesla MRI identified additional lesions not seen on lower-field MRI in nine patients (23\%), which guided further surgical decision making. Six of these patients had been operated at time of the study, with confirmation of FCD ILAE type IIA ( $n=2), F C D$ IIB $(n=1)$, mMCD type II $(n=2)$ or mMCD with oligodendroglial hyperplasia (MOGHE) $(n=1)$.

During assessment of 7T MRI scans that had been performed on clinical indication, we noticed an emerging pattern of a specific signal change co-localizing with confirmed $\mathrm{mMCD} / \mathrm{FCD}$ lesions. These observations in a series of six patients are described in Chapter 5. In four of six patients in our series, 7T $T_{2}{ }^{*}$ sequences showed areas containing marked hypointensities with a branched, partly tortuous configuration, and a signal compatible with venous blood, suggestive of increased venous vasculature in the sulci neighbouring the malformed cortex and to a lesser extent in brain parenchyma. In two of these patients 3T MRI was without abnormalities and in one also 7T MRI did not reveal a lesion.

We reviewed 7T images of eight healthy subjects, and found that symmetrical venous leptomeningeal vascular structures were clearly visible but the phenomenon described above was not identified.

In Chapter $\mathbf{6}$ we set out to elucidate the relation between micro-vascular density and cortical developmental abnormalities, in order to determine whether there is a pathological substrate for imaging findings compatible with vascular changes. We performed quantitative immunohistochemical analysis of microvascular density and inflammation in 97 patients with either mMCD, FCD or TSC and an age- and localization-matched control group of 14 autopsy cases. We found that cortical and white matter vascular density is increased in FCD and tubers, compared to the surrounding perilesional cortex, irrespective of age. As opposed to normal cortex, in resected malformations of cortical development - most pronounced in cortical tubers - we observed an increase in vascular density with ageing. No time-relation was seen in white matter vascular density. 
Moreover, vessel density in white matter was related to pathology specific processes. Compared to controls, there was increased density in TSC tubers. Hypervascularity in tubers was associated with microglial activation, and an increased number of inflammatory cells. Vascular density was not significantly different between FCD subtypes and controls, neither did we find an association between cortical vascular density and pathology subtypes.

The first clinical experiences of 7T MRI were promising, however it was clear that in the majority of patients with normal 3T MRI, neither did 7T MRI lead to identification of a lesion. The preceding chapters underline the importance of pre-surgical lesion identification and delineation and accurate histopathological diagnosis. Further progress requires analysis tools that correlate 7T image and histological characteristics. In the course of this PhD project the prospective CODE 7 study was initiated and data collection performed. Candidates for epilepsy surgery with suspected mMCD/FCD were included and underwent additional pre-surgical 7T MRI. The resected brain specimens were studied with ex vivo 7T MRI, in isotropic resolutions up to $0.2 \mathrm{~mm}$. The in vivo and ex vivo MR images were co-registered with digitized histology slides with the use of in-house developed software tools. The development of this methodological framework is described in Chapter 7. These preliminary results appear promising, and we in ongoing research we pursue to elucidate how specific MR imaging features relate to histological characteristics in an extensive set of stainings.

\section{General Discussion}

The first two chapters of this thesis describe how clinical characteristics and both seizure and cognitive outcome after epilepsy surgery relate to histopathological diagnoses as defined by the 2011 ILAE classification for $\mathrm{mMCD} / \mathrm{FCD}^{13}$. The work bundled in the second half of this thesis focuses on our experiences with 7T MRI in this patient group and the pursuit in improving lesion detection and delineation.

\section{Outcome differences between histological subtypes}

There is substantial disparity in $\mathrm{mMCD} / \mathrm{FCD}$ literature with regards to the frequency of occurrence of the histological subtypes and their relation with several clinical parameters, also in recent publications that applied identical FCD classifications. A possible factor is that observer variability remains despite efforts to harmonize histopathological classification. Discrepancy in utilization of immunohistochemical methods may cause variability in subclassification.

Especially the milder pathologies can be debatable. There was no formal inclusion of $\mathrm{mMCD}$ in the 2011 ILAE classification system, which might persuade the pathologist to classify very subtle dysplastic lesions as FCD I, when mMCD would be more suitable, leading to less awareness of, or attention for $\mathrm{mMCD}$ as an entity. Consequently in the last years mMCD has 
been rarely reported on, where it used to be a common diagnosis after epilepsy surgery, particularly in children ${ }^{10}$.

In recent literature, there is inconsistency in the reported correlation between ILAE FCD subtypes and clinical presentation and surgical outcome. Multiple studies did not find differences in clinical presentation ${ }^{44,168,169}$ or surgical outcome ${ }^{35-37,41,168-170}$ between FCD subtypes. Studies that did report different clinical characteristics found more MRI negative lesions and later onset of epilepsy in patients with FCD I compared to those with FCD II diagnoses $35,40,171$. FCD I lesions are more frequently multilobar while in FCD II there was a predilection for frontal localization ${ }^{35,171}$. Several studies found better surgical outcome in the FCD II subtypes, with highest chance of seizure freedom after a FCD IIb diagnosis 28,40,44,171-173.

We studied a large cohort of children operated from 2000 to2012 for whom histology was revised according the ILAE classification, with isolated $\mathrm{mMCD}$ or FCD. In both cognitive and seizure outcome we observed differences between histological subtypes.

Seizure freedom following surgery is less likely in $\mathrm{mMCD}$, probably due to difficulties in determining location and margins on imaging and during surgery, thus complicating accurate lesion identification, delineation, and complete removal of the epileptogenic zone. In our study we did not observe differences between histological subtypes, in occurrence of suspected incomplete resection based on intra-operative observations by the surgeon, ECOG or post-surgical imaging. Nonetheless residual lesions are most likely the best explanation for unfavourable seizure outcome in patients with mMCD or FCD. Complete resection is the most reported predictor of seizure-freedom in literature, along with factors that relate to completeness of resection: a temporal focus, severe pathological features, and an MRIdefined lesion ${ }^{43}$. Accordingly, in our multivariate analyses, $\mathrm{mMCD}$, extra-temporal surgery and an indication for incomplete resection were all predictors of unfavourable seizure outcome. Consistent with existing literature, $\mathrm{mMCD}$ and $\mathrm{FCD}$ I remained more frequently undetected on MRI than FCD II35,37,42,70,75. However, in our cohort absence of an MRI lesion did not appear to negatively influence surgical outcome. Over half of the studied patients underwent long-term invasive monitoring, needed to localize and delineate of the epileptic zone in patients without identified structural lesion and facilitate complete removal.

While seizure freedom rates are lower compared to FCD II subtypes, surgery is also worthwhile for patients suspected with the more subtle mMCD or FCD I subtypes. Over the last decades we have witnessed an increasing trend to operate on MRI-negative cases ${ }^{64}$. Nevertheless we should strive to improve localisation and delineation of especially mMCD and FCD I to improve surgical outcome. There is still debate concerning the definitive identifying contrasting histological and clinical features of mMCD versus FCD ${ }^{74}$. Our findings do suggest that clinical characteristics differentiate patients with $\mathrm{mMCD}$ subtypes and FCD. 
Future studies should continue to include $\mathrm{mMCD}$ pathology to improve understanding of clinical distinctions. Well-coordinated long-term prospective multicentre studies seem necessary to definitely establish predictors of seizure outcome and build predictive models in patients with $\mathrm{mMCD} / \mathrm{FCD}$. A prerequisite is high inter-rater reliability of histological evaluation and classification. Future studies could potentially further differentiate $\mathrm{mMCD}$ and FCD subtypes through genetic and molecular fingerprinting ${ }^{74}$. For example, mTOR pathway pathogenic variants appear to be reserved for FCD II subtypes, while brain-only loss-of-function SLC35A2 mutations were exclusively found in $\mathrm{mMCD/FCD} \mathrm{in} \mathrm{a} \mathrm{single}$ study ${ }^{174}$. It is not unlikely that genetic information will further change the classification of focal developmental abnormalities.

Previous research showed that developmental delay is common among patients with epilepsy due to $F C D$, with $53-68 \%$ having an IQ below $70^{27,28}$. Most studies reported overall cognitive improvement following epilepsy surgery ${ }^{45-48}$, and factors related to post-surgical cognitive outcome were seizure freedom ${ }^{28,48-51}$, parental education ${ }^{52}$, anti-epileptic drug (AED) policies ${ }^{51,53}$, preoperative seizure frequency ${ }^{27}$, and aetiology $y^{53,54}$. More severe cognitive impairment was predicted by earlier onset ${ }^{27,46,55-62}$ and longer duration ${ }^{27,63}$ of epilepsy.

Previous studies did not find differences in cognitive outcome between patients with different $\mathrm{FCD}$ subtype ${ }^{27,42}$.In our cohort, cognitive disability was slightly more prevalent (74\%) than expected. We confirmed the association of early onset of epilepsy with cognitive impairment in patients with $\mathrm{mMCD} / \mathrm{FCD}$. Also longer epilepsy duration negatively affected cognition. Cognitive outcome was strongly related to pre-surgical functioning.

Epileptic activity, even when associated with strictly focal semiology, causes disruption of cognitive development and memory functions. In these patients there is evidence of loss of optimal, small-world, network configuration and of structural white matter abnormalities, wide spread and distant to the epileptogenic focus ${ }^{30,31,80,81}$. In addition, many anti-epileptic drugs are associated with cognitive problems, due to a generalized suppressive effect with alteration of neuronal excitability ${ }^{80,82}$. Consequently, longer exposure to epileptic activity and AEDs would reasonably increase the gap in cognitive function with normal developing peers. Another factor that might contribute to a more pronounced cognitive delay with earlier epilepsy onset, is that there is evidence of a critical period in cognitive development during the first years of life, when the foundation is laid for development of higher-order skills later in life ${ }^{60}$. Alternatively, both a lower IQ and earlier onset epilepsy could be the consequence of a more severe or more widespread underlying epileptogenic pathology, rather than being causally related ${ }^{61}$. FCD I is typically more diffuse than other FCD subtypes and in our study patients with FCD I had more severe cognitive delay, although they did not present with earlier seizure onset. 
We expected to find more cognitive improvement in seizure - and medication-free patients but could not confirm this association in our study, in contrast to several previous studies ${ }^{49-51}$. These studies had a markedly longer follow-up duration. We observed overall improvement in cognition, but it is likely that follow-up exceeding two years is required to appreciate post-surgical long-term positive effects of seizure freedom and cessation of anti-epileptic drugs on cognitive development.

There is a shift towards epilepsy surgery at younger age, especially for patients with focal developmental abnormalities. In the pediatric population improvement of cognitive functioning should be regarded as a significant aim of surgical treatment. Preservation of cognitive abilities is a strong argument for streamlining the pre-surgical diagnostic trajectory. We should strive to minimize delay between onset of epilepsy and surgery in surgical candidates, while safeguarding the quality of the diagnostic process. Disease duration has significantly decreased over the last decades, but as an European survey points out there are still massive gains to be made, with a mean epilepsy duration of 7.7 years in children and 24.4 years in adults in the last decade ${ }^{64}$.

\section{Ultra-High field MR imaging}

Improving lesion detection and delineation is one of the ways to improve quality of care for epilepsy surgery patients. One technical development that has potential is 7 tesla (T) MRI. Current standard of care is the use of an epilepsy-dedicated scanning protocol on 1.5 or 3 tesla. Higher field strength increases the signal to noise ratio, which allows higher image resolution, or faster acquisition. Such theoretical advantage will not unquestionably translate to clinical benefits.

In 2017 the first 7T MRI system received clearance for clinical use. Meanwhile clinical systems with 9.4T, 10.5T and 11.7 T magnets have been developed and await approval for clinical use. These systems make scanning at isotropic resolutions under half a millimetre possible. There will be a limit to further increasing field strength as more severe physical sensations, or unacceptable high SAR-levels form unsurmountable obstacles at some point. Animal studies found no evidence of short or long term irreversible effects with exposure to 11.1T, 17.6T and 21.1T magnetic fields for over one hour. However, possible detrimental effects of 10T fields and beyond on replicating cells and growing tissues require further investigation ${ }^{175}$.

Increasing field strength is not the only way to improve resolving power of MRI. Other groups have used anaesthesia to be able to increase acquisition times to over an hour, increasing maximal resolution and attenuating motion artefacts on conventional MRI systems. General anaesthesia to undergo imaging is not without risks and requires additional staff, incurring high costs. Rather technical improvements should be sought for increasing resolution. Besides increasing field strength, there are potential gains by for instance using increasingly 
sophisticated multi-channel RF coils, parallel scanning techniques allowing faster acquisition and more efficient pulse designs ${ }^{112,176,177}$. Further improvements in spatial resolutions can be expected to result in an increase in sensitivity for subtle cortical abnormalities. However, epilepsy surgery has seen a trend towards epilepsy surgery in increasingly complex cases, which also include more cryptogenic epilepsies. Increasing sensitivity caries a risk of decreasing specificity; limiting false positive MRI findings will be a challenge. Integration of diagnostic modalities is quintessential to address this potential pitfall.

In our 7T MRI cohort (chapter 4) no lesion was identified prior to surgery using standard epilepsy dedicated (1-3T) MRI protocols in 9/57 (16\%) of patients with FCD and 14/31 (45\%) of patients mMCD. This is in line with previous studies ${ }^{37,42,70}$. Multidisciplinary evaluation of 7T MRI led to identification of a structural lesion which had not been found with standard epilepsy MRI studies in $23 \%$ of patients. These findings were guiding in further clinical decision making. Other groups have now reported similar yield of 7T MRI 99,156,178.

However, in 77\% of the studied cohort, even 7T MRI did not reveal a lesion. Several patients have already undergone surgery at time of this study, and in some a mMCD/FCD was demonstrated with histopathological investigation. It is clear 7T MRI is not a panacea. Experience with 7T MRI is still relatively limited. The biggest advantage, added anatomical detail, can paradoxically also be an extra challenge in differentiating between meaningless variation and true pathology. Moreover, 7T examinations can produce datasets of thousands of slices, incurring proportionately longer visual assessment time. It is to be expected that with growing experience accuracy of visual assessment by radiologist will improve.

In the last decade there has been a swiftly growing interest in using post-processing techniques as an adjunct to conventional visual analyses. Numerous techniques have been described of which most are based on voxel-based morphometry (VBM) or surface anatomy analyses. Several studies have shown that post-processing can lead to identification of subtle abnormalities even after reading by specialised neuroradiologists and multidisciplinary review failed to identify a lesion ${ }^{179-185}$. Moreover, studies found an association between complete resection of retrospectively flagged regions and favourable seizure outcome ${ }^{181,183}$. Scrutiny is essential concerning the results of these analyses since cut-off values that are able to detect subtle abnormalities will inherently have low specificity and thus carry a risk of false positivity ${ }^{186}$. Nevertheless, also in a recent prospective study morphometric analyses were found to impact on management and aid in detection of subtle FCD's ${ }^{187}$. To date, these computer-aided analyses have been restricted to 1.5 and 3 tesla acquisitions. In general, a sufficiently large reference data set is required with ideally identical image specifications, and performance is also dependent on proper age-matching. These analyses are also hampered by the challenges related to image inhomogeneity at 7T. However, In the near future there could be a place for morphometric analyses and Artificial Intelligence 
algorithms in flagging potential lesions in the extensive 7T MRI datasets, hereby shortening reading times and increasing clinical yield.

Even though the brain is a relatively stationary organ, at submillimetre resolution even the slightest motion produces artefacts, for instance those that are due to arterial pulsation. Inherent to incremental field strength is an increase in artefacts due to magnetic susceptibility and field inhomogeneities. Especially signal drop-out in the temporal lobes has hampered clinical evaluation of epilepsy patients. Several techniques have been developed that successfully counter artefacts due to field inhomogeneity and susceptibility effects ${ }^{188}$. Motion artefacts can be reduced by implementation of specific pulse sequences that track movement during image acquisition ${ }^{189}$. These developments greatly improved temporal lobe visualisation at 7T MRI. Concludingly, accuracy of 7T MRI is expected to improve with growing experience and technological advancement.

What is the current place of 7T MRI in the diagnostic work-up for epilepsy surgery in patients suspected of subtle cortical developmental anomalies? In patients with focal epilepsy and normal MRI findings on standard MRI investigations, $\mathrm{mMCD}$ and FCD I are frequently the underlying cause. If available 7 tesla MRI should be considered. We argue there is clinical benefit in applying scanning protocols with isotropic resolutions of 0.5 to $1 \mathrm{~mm}$ for $T_{1^{\prime}} T_{2}$ and FLAIR sequences. Careful consideration concerning the indication is warranted since availability of 7T MRI will remain relatively limited and its operational costs higher compared to standard 1.5 or 3T MRI. There should be a convincing hypothesis for focal onset of seizures, based on semiology and EEG, and either 1.5 or 3T MRI should have been performed using an epilepsy dedicated protocol and discussed in multidisciplinary meetings, and not have revealed an explanatory lesion or have ambiguous findings. Non-structural causes of focal refractory epilepsy - such as certain genetic etiologies - should have been excluded and a high index of suspicion for a structural underlying cause is required. If available, computerized post-processing MRI analysis methods should have been applied. Still, it is to be expected that in a majority of these casus 7T MRI will not provide information that contributes to a stronger hypothesis.

Our findings described in chapter $\mathbf{5}$ are a product of the pursuit for alternative imaging characteristics to localise subtle cortical dysplasias. We hypothesized that these $T_{2}{ }^{*}$ signal changes at 7T MRI could be a reflection of structural vascular changes, either developmental or acquired and related to epileptic activity. Alternatively, hemodynamic alterations and changes in blood oxygen levels could play a role.

The histological study that followed did not provide conclusive support for vascular structural changes as a substrate of $T_{2}{ }^{*}$ findings. Importantly, however, leptomeningeal vasculature which could be related to the observed $T_{2}{ }^{*}$ changes - was not studied histologically since this part of the removed tissue is rarely available for histopathological review. FCD did not 
appear to be hypervascular compared to controls, even though there was an increase in the vascular density in the lesion compared to perilesional tissue. Hemodynamic and consequent blood oxygen level variations, perhaps in meningeal vascular structures, possibly better explain vascular prominence on $T_{2}{ }^{*} \mathrm{MR}$ images. Both positive and negative BOLD responses have been shown to be concordant with proven FCD lesions and also seemed to correspond with epileptogenic tissue in MRI negative patients ${ }^{190}$. Future research is needed to ascertain the value of BOLD fMRI in detection of otherwise MRI negative dysplasia.

It remains unclear what is required to approach $100 \%$ lesion detection on MRI, if ever feasible. Should the focus be to improve spatial resolution or improve tissue contrast? Or should scanning techniques be altered to optimize specific tissue contrast based on histological changes in the targeted lesion type? These are questions that led to the start of our study in which we performed ex-vivo/histology co-registered analyses, to allow direct comparison of MR signal and histological characteristics. Analyses of the findings of this study - currently work in progress-hopefully aids in improving lesion identification and delineation.

Structural MRI is still the keystone imaging modality for patients with epilepsy, however other techniques could potentially gain importance in the future. Nuclear imaging for epilepsy in the form of PET-CT and SPECT are established and commonly used techniques to provide information on tissue involved in epileptogenesis or propagation through visualisation of respectively metabolic activity or perfusion differences. Few epilepsy centres employ MR spectroscopy or MR spectroscopic imaging which can also reveal metabolic changes in patients with epilepsy ${ }^{100}$. These techniques are frequently used to study brain tumours and neurodegenerative disorders, but so far relatively little research has focused on epilepsy. Single- and multi-voxels studies have proven able to lateralise epileptic - in particular hippocampal - foci and demonstrate epileptogenicity in known structural abnormalities, based on abnormal metabolite concentrations- $\mathrm{N}$-acetyl aspartate, choline and creatine especially. However, voxel-based MRS is of little value in MRI-negative patients with a suspected neocortical focus ${ }^{191}$. MR spectroscopic imaging (MRSI) can potentially provide full brain coverage, but its use is currently limited by technical limitations in e.g. spatial resolution, acquisition times, detector sensitivity and artefacts related to field inhomogeneity. Recent developments in acquisition techniques in combination with higher field strength bring within reach MRSI that could be clinically useful in detection of epileptogenic, but MRI-occult, dysplastic lesions ${ }^{192}$. Future research in this field demands special attention.

\section{Final Remarks}

Although patients with most subtle abnormalities in the spectrum of mMCD/FCD have a significantly lower chance of achieving seizure freedom, still one third of these patients are expected to reach complete and ongoing seizure freedom. Suspected mMCD should not deter physicians to offer surgery as option. We found that patients with mMCD clinically 
differ from those with ILAE FCD I and FCD II subtypes and advocate to maintain mMCD as a separate entity and continue to study this patient group in future research. An iterative histopathological agreement trial to identify areas of diagnostic challenges within the present classification scheme is ongoing (FCD classification Task Force of ILAE). Developmental delay forms a significant burden of disease in patients with refractory epilepsy. Cognitive functioning can significantly improve after resective epilepsy surgery in patients with $\mathrm{mMCD} / \mathrm{FCD}$. Moreover, cognitive delay builds up with the duration a patient suffers from epilepsy. This is one of the reasons to be swift in determining candidacy for surgical therapy. Surgical care for epilepsy patients can be further improved by progress in imaging of potential underlying structural abnormalities, such as the often highly subtle lesions associated with $\mathrm{mMCD} / \mathrm{FCD}$. Our work showed that the clinical use of 7T MRI increases identification of subtle lesions and can be a valuable tool in the pre-surgical work-up. However, this technique as it is, still has far from perfect diagnostic accuracy. Increasing imaging resolution could improve lesion detection, facilitated by technical advance in field strength, coil technique and image reconstruction. Research should also focus on additional localising structural and possibly functional MR image features. Optimal care will not only come from these technical innovations but also the integration of data of the different ancillary tests, and from the multidisciplinary approach with neurologists, electrophysiologists, neurosurgeons, technical physicians, neuropsychologists and radiologists closely working together. Patients with refractory epilepsy, relatives and caregivers often experience tremendous burden of disease. Epilepsy surgery is arguably one of the most rewarding therapies in modern neurology, and fortunately we can expect continuing improvement of the standard of care, due to worldwide intense efforts by scientists and healthcare professionals. 


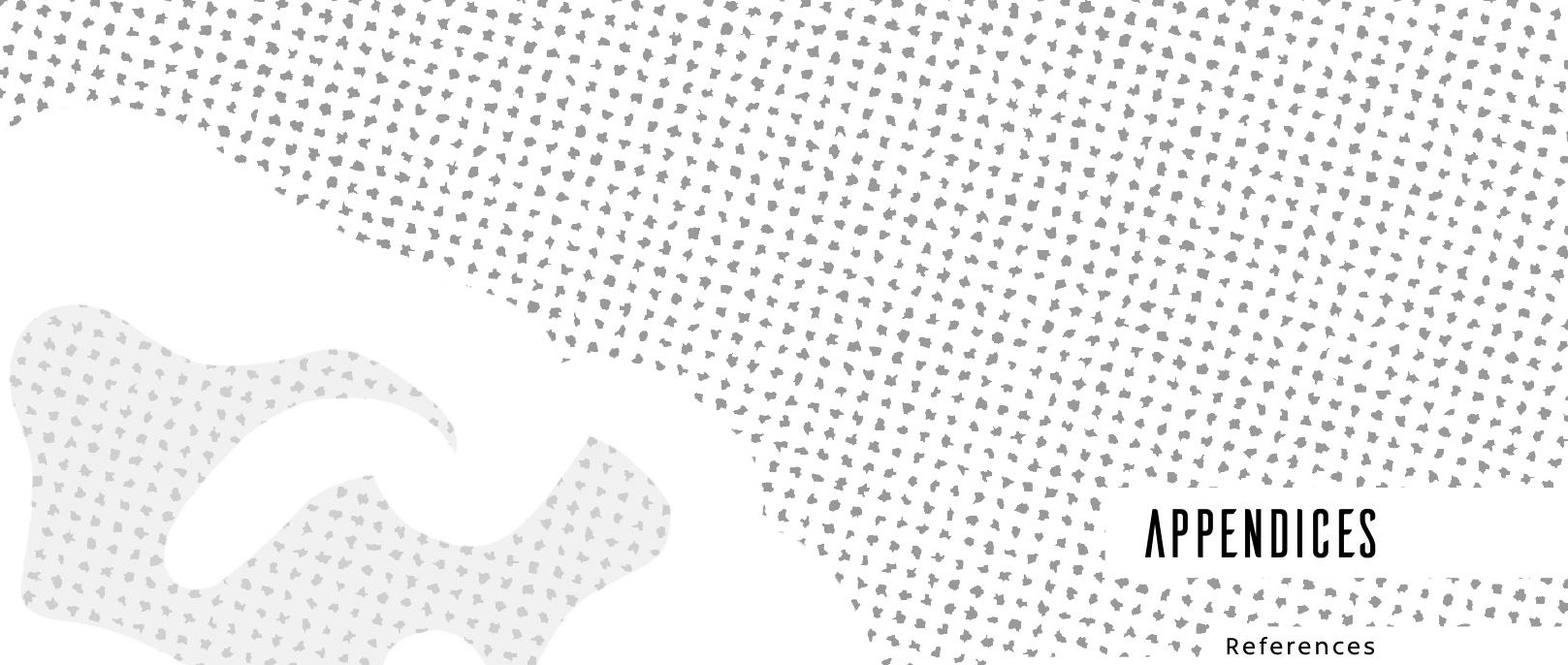

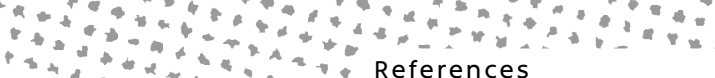
Nederlandse samenvatting + Acknowledgements * - Curriculum Vitae

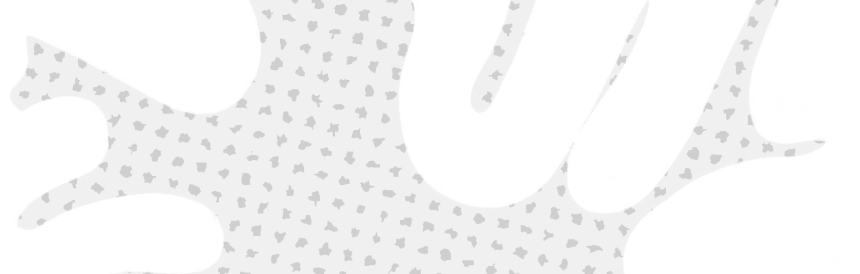
List of publications

$4+40+4$

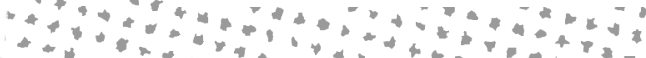
$+\infty$

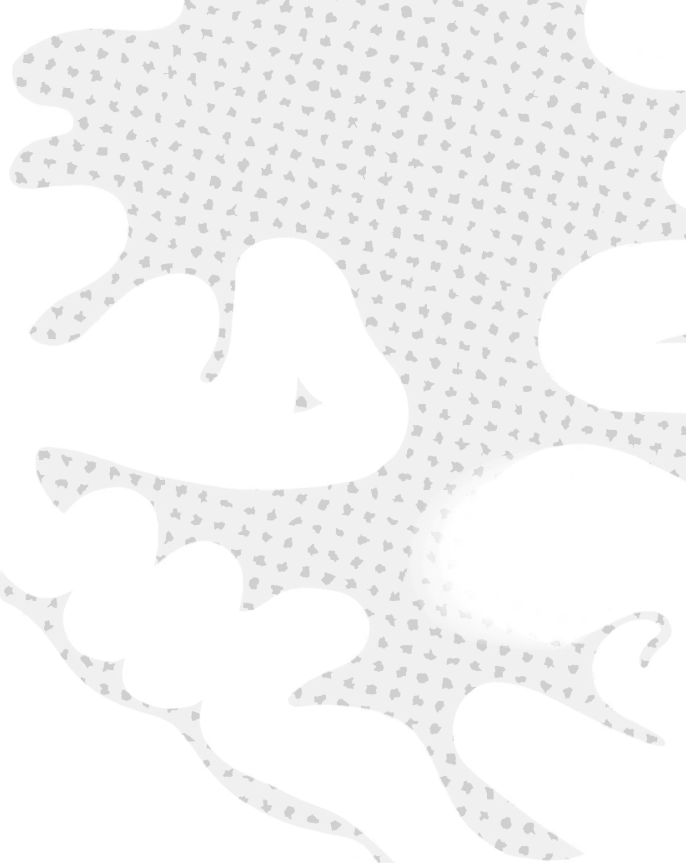




\section{References}

1. Beghi E, Berg A, Carpio A, Forsgren L, Hesdorffer DC, Hauser WA, et al. Epileptic Seizures and Epilepsy: definitions proposed by the International League Against Epilepsy (ILAE) and the International Bureau for Epilepsy (IBE). Epilepsia. 2005 Oct;46(10):1698-9; author reply 1701-2.

2. Fisher RS, Acevedo C, Arzimanoglou A, Bogacz A, Cross JH, Elger CE, et al. ILAE Official Report: A practical clinical definition of epilepsy. Epilepsia. 2014;55(4):475-82.

3. Scheffer IE, Berkovic S, Capovilla G, Connolly MB, French J, Guilhoto L, et al. ILAE classification of the epilepsies: Position paper of the ILAE Commission for Classification and Terminology. Epilepsia. 2017;58(4):512-21.

4. Krumholz A, Wiebe S, Gronseth GS, Gloss DS, Sanchez AM, Kabir AA, et al. Evidence-based guideline: Management of an unprovoked first seizure in adults: Report of the Guideline Development Subcommittee of the American Academy of Neurology and the American Epilepsy Society. Epilepsy Curr. 2015;15(3):144-52.

5. Shellhaas RA, Berg AT, Grinspan ZM, Wusthoff CJ, Millichap JJ, Loddenkemper T, et al. Initial Treatment for Nonsyndromic Early-Life Epilepsy: An Unexpected Consensus. Pediatr Neurol [Internet]. 2017 Oct;75(1):73-9.

6. Kwan P, Brodie MJ. Early identification of refractory epilepsy. N Engl J Med. 2000 Feb 3;342(5):314-9.

7. Picot $M-C$, Baldy-Moulinier M, Daurès J-P, Dujols $P$, Crespel $A$. The prevalence of epilepsy and pharmacoresistant epilepsy in adults: a population-based study in a Western European country. Epilepsia [Internet]. 2008 Jul;49(7):1230-8.

8. Cockerell OC, Johnson AL, Sander JW, Hart YM, Shorvon SD. Remission of epilepsy: results from the National General Practice Study of Epilepsy. Lancet (London, England) [Internet]. 1995 Jul 15;346(8968):140-4.

9. Kwan P, Arzimanoglou A, Berg AT, Brodie MJ, Allen Hauser W, Mathern G, et al. Definition of drug resistant epilepsy: consensus proposal by the ad hoc Task Force of the ILAE Commission on Therapeutic Strategies. Epilepsia. 2010 Jun;51(6):1069-77.

10. Blumcke I, Spreafico R, Haaker G, Coras R, Kobow K, Bien CG, et al. Histopathological Findings in Brain Tissue Obtained during Epilepsy Surgery. N Engl J Med. 2017 Oct 26;377(17):1648-56.

11. Blümcke I, Spreafico R. An international consensus classification for focal cortical dysplasias. Lancet Neurol. 2011 Jan;10(1):26-7.

12. Mühlebner A, Coras R, Kobow K, Feucht M, Czech T, Stefan H, et al. Neuropathologic measurements in focal cortical dysplasias: validation of the ILAE 2011 classification system and diagnostic implications for MRI. Acta Neuropathol. 2012 Feb 27;123(2):259-72.

13. Blümcke I, Thom M, Aronica E, Armstrong DD, Vinters HV, Palmini A, et al. The clinicopathologic spectrum of focal cortical dysplasias: A consensus classification proposed by an ad hoc Task Force of the ILAE Diagnostic Methods Commission. Epilepsia. 2011 Jan;52(1):158-74. 
14. Palmini A, Najm I, Avanzini G, Babb T, Guerrini R, Foldvary-Schaefer N, et al. Terminology and classification of the cortical dysplasias. Neurology. 2004 Mar 23;62(6 Suppl 3):S2-8.

15. Taylor DC, Falconer MA, Bruton CJ, Corsellis JA. Focal dysplasia of the cerebral cortex in epilepsy. J Neurol Neurosurg Psychiatry. 1971;34(4):369-87.

16. Blümcke I, Coras R. The curse of in silico transformation from Palmini's into the ILAE classification system of focal cortical dysplasia: A critical comment. Epilepsia. 2013;54(8):1506-7.

17. Aronica E, Mühlebner A. Neuropathology of epilepsy. Handb Clin Neurol. 2017;145(1):193-216.

18. Laplante M, Sabatini DM. mTOR signaling at a glance. J Cell Sci. 2009;122(20):3589-94.

19. Mühlebner A, Bongaarts A, Sarnat HB, Scholl T, Aronica E. New insights into a spectrum of developmental malformations related to mTOR dysregulations: challenges and perspectives. J Anat. 2019;235(3):521-42.

20. Palmini $\mathrm{A}$, Holthausen $\mathrm{H}$. Focal malformations of cortical development: a most relevant etiology of epilepsy in children. 1st ed. Vol. 111, Handbook of clinical neurology. Elsevier B.V.; 2013. 549-65

21. Najm IM, Tassi L, Sarnat HB, Holthausen H, Russo G Lo. Epilepsies associated with focal cortical dysplasias (FCDs). Acta Neuropathol. 2014 Jun 12;

22. Keezer MR, Sisodiya SM, Sander JW. Comorbidities of epilepsy: Current concepts and future perspectives. Lancet Neurol. 2016;15(1):106-15.

23. Seidenberg M, Pulsipher DT, Hermann B. Association of epilepsy and comorbid conditions. Future Neurol. 2009;4(5):663-8.

24. Dalmagro CL, Velasco TR, Bianchin MM, Martins APP, Guarnieri R, Cescato MP, et al. Psychiatric comorbidity in refractory focal epilepsy: A study of 490 patients. Epilepsy Behav. 2012;25(4):593-7.

25. Jansen C, Francomme L, Vignal JP, Jacquot C, Schwan R, Tyvaert L, et al. Interictal psychiatric comorbidities of drug-resistant focal epilepsy: Prevalence and influence of the localization of the epilepsy. Epilepsy Behav. 2019;94:288-96.

26. Hellwig S, Mamalis P, Feige B, Schulze-Bonhage A, van Elst LT. Psychiatric comorbidity in patients with pharmacoresistant focal epilepsy and psychiatric outcome after epilepsy surgery. Epilepsy Behav. 2012;23(3):272-9.

27. Kimura N, Takahashi Y, Shigematsu H, Imai K, Ikeda H, Ootani H, et al. Risk factors of cognitive impairment in pediatric epilepsy patients with focal cortical dysplasia. Brain Dev. 2018;

28. Kwon HE, Eom S, Kang H, Lee JS, Kim SH, Kim DS, et al. Surgical treatment of pediatric focal cortical dysplasia: Clinical spectrum and surgical outcome. Neurology. 2016 Aug 30;87(9):945-51.

29. Holley AJ, Hodges SL, Nolan SO, Binder M, Okoh JT, Ackerman K, et al. A single seizure selectively impairs hippocampal-dependent memory and is associated with alterations in PI3K/Akt/mTOR and FMRP signaling. Epilepsia Open. 2018;3(4):511-23.

30. van Diessen E, Zweiphenning WJEM, Jansen FE, Stam CJ, Braun KPJ, Otte WM. Brain Network Organization in Focal Epilepsy: A Systematic Review and Meta-Analysis. PLoS One. 2014;9(12):e114606.

31. Otte WM, Dijkhuizen RM, van Meer MPA, van der Hel WS, Verlinde SAMW, van Nieuwenhuizen $\mathrm{O}$, et al. Characterization of functional and structural integrity in experimental focal epilepsy: Reduced network efficiency coincides with white matter changes. PLoS One. 2012;7(7). 
32. Braun KPJ. Preventing cognitive impairment in children with epilepsy. Curr Opin Neurol. 2017 Aug;30(2):140-7.

33. West S, Nolan SJ, Cotton J, Gandhi S, Weston J, Sudan A, et al. Surgery for epilepsy. In: West S, editor. Cochrane Database of Systematic Reviews. Chichester, UK: John Wiley \& Sons, Ltd; 2015.

34. Lüders HO, Najm I, Nair D, Widdess-Walsh P, Bingman W. The epileptogenic zone: general principles. Epileptic Disord. 2006 Aug;8 Suppl 2(SUPPL. 2):S1-9.

35. Isler C, Kucukyuruk B, Ozkara C, Gunduz A, Is M, Tanriverdi T, et al. Comparison of clinical features and surgical outcome in focal cortical dysplasia type 1 and type 2. Epilepsy Res. 2017 Oct;136(April):130-6.

36. Jin B, Wang J, Zhou J, Wang S, Guan Y, Chen S. A longitudinal study of surgical outcome of pharmacoresistant epilepsy caused by focal cortical dysplasia. J Neurol. 2016 Dec 8;263(12):240310.

37. Fauser S, Essang C, Altenmüller D-M, Staack AM, Steinhoff BJ, Strobl K, et al. Long-term seizure outcome in 211 patients with focal cortical dysplasia. Epilepsia. 2015 Jan 13;56(1):66-76.

38. Mrelashvili A, Witte RJ, Wirrell EC, Nickels KC, Wong-Kisiel LC. Seizure Freedom in Children With Pathology-Confirmed Focal Cortical Dysplasia. Pediatr Neurol. 2015 Dec;53(6):513-8.

39. Krsek P, Maton B, Jayakar P, Dean P, Korman B, Rey G, et al. Incomplete resection of focal cortical dysplasia is the main predictor of poor postsurgical outcome. Neurology. 2009 Jan 20;72(3):217-23.

40. Kim DW, Lee SK, Chu K, Park KI, Lee SY, Lee CH, et al. Predictors of surgical outcome and pathologic considerations in focal cortical dysplasia. Neurology. 2009 Jan 20;72(3):211-6.

41. Mühlebner A, Gröppel G, Dressler A, Reiter-Fink E, Kasprian G, Prayer D, et al. Epilepsy surgery in children and adolescents with malformations of cortical development-outcome and impact of the new ILAE classification on focal cortical dysplasia. Epilepsy Res. 2014 Nov 30;108(9):1652-61.

42. Krsek P, Maton B, Korman B, Pacheco-Jacome E, Jayakar P, Dunoyer C, et al. Different features of histopathological subtypes of pediatric focal cortical dysplasia. Ann Neurol. 2008 Jun;63(6):758-69.

43. Rowland NC, Englot DJ, Cage T a, Sughrue ME, Barbaro NM, Chang EF. A meta-analysis of predictors of seizure freedom in the surgical management of focal cortical dysplasia. J Neurosurg. 2012 May;116(5):1035-41.

44. Choi SA, Kim SY, Kim H, Kim WJ, Kim H, Hwang H, et al. Surgical outcome and predictive factors of epilepsy surgery in pediatric isolated focal cortical dysplasia. Epilepsy Res. 2018 Jan;139(November 2017):54-9.

45. Sherman EMS, Wiebe S, Fay-McClymont TB, Tellez-Zenteno J, Metcalfe A, Hernandez-Ronquillo $L$, et al. Neuropsychological outcomes after epilepsy surgery: Systematic review and pooled estimates. Epilepsia. 2011;52(5):857-69.

46. Sibilia V, Barba C, Metitieri T, Michelini G, Giordano F, Genitori L, et al. Cognitive outcome after epilepsy surgery in children: A controlled longitudinal study. Epilepsy Behav. 2017;73:23-30.

47. Skirrow C, Cross JH, Cormack F, Harkness W, Vargha-Khadem F, Baldeweg T. Long-term intellectual outcome after temporal lobe surgery in childhood. Neurology. 2011;76(15):1330-7.

48. Lee YJ, Lee JS, Kang HC, Kim DS, Shim KW, Eom S, et al. Outcomes of epilepsy surgery in childhoodonset epileptic encephalopathy. Brain Dev. 2014;36(6):496-504. 
49. Puka K, Tavares TP, Smith M Lou. Development of intelligence 4 to 11 years after paediatric epilepsy surgery. J Neuropsychol. 2017;11(2):161-73.

50. Puka K, Smith M Lou. Academic skills in the long term after epilepsy surgery in childhood. Epilepsy Behav. 2016;62:97-103.

51. Hallböök T, Tideman P, Rosén I, Lundgren J, Tideman E. Epilepsy surgery in children with drugresistant epilepsy, a long-term follow-up. Acta Neurol Scand. 2013;128(6):414-21.

52. Meekes J, Van Schooneveld MMJ, Braams OB, Jennekens-Schinkel A, Van Rijen PC, Hendriks $\mathrm{MPH}$, et al. Parental education predicts change in intelligence quotient after childhood epilepsy surgery. Epilepsia. 2015;56(4):599-607.

53. Boshuisen K, van Schooneveld MMJ, Uiterwaal CSPM, Cross JH, Harrison S, Polster T, et al. Intelligence quotient improves after antiepileptic drug withdrawal following pediatric epilepsy surgery. Ann Neurol. 2015 Jul;78(1):104-14.

54. Puka K, Rubinger L, Chan C, Smith M Lou, Widjaja E. Predictors of intellectual functioning after epilepsy surgery in childhood: The role of socioeconomic status. Epilepsy Behav. 2016;62:35-9.

55. Vendrame M, Alexopoulos A V., Boyer K, Gregas M, Haut J, Lineweaver T, et al. Longer duration of epilepsy and earlier age at epilepsy onset correlate with impaired cognitive development in infancy. Epilepsy Behav. 2009;16(3):431-5.

56. van Iterson L, Zijlstra BJH, Augustijn PB, van der Leij A, de Jong PF. Duration of epilepsy and cognitive development in children: A longitudinal study. Neuropsychology. 2014;28(2):212-21.

57. Kaaden S, Helmstaedter C. Age at onset of epilepsy as a determinant of intellectual impairment in temporal lobe epilepsy. Epilepsy Behav. 2009;15(2):213-7.

58. Berg AT, Zelko FA, Levy SR, Testa FM. Age at onset of epilepsy, pharmacoresistance, and cognitive outcomes: a prospective cohort study. Neurology. 2012;79(13):1384-91.

59. Hermann B, Seidenberg M, Bell B, Rutecki P, Sheth R, Ruggles K, et al. The neurodevelopmental impact of childhood-onset temporal lobe epilepsy on brain structure and function. Epilepsia. 2002;43(9):1062-71.

60. Cormack F, Helen Cross J, Isaacs E, Harkness W, Wright I, Vargha-Khadem F, et al. The development of intellectual abilities in pediatric temporal lobe epilepsy. Epilepsia. 2007;48(1):201-4.

61. Huttenlocher PR, Hapke RJ. A follow-up study of intractable seizures in childhood. Ann Neurol. 1990 Nov;28(5):699-705.

62. Vasconcellos E, Wyllie E, Sullivan S, Stanford L, Bulacio J, Kotagal P, et al. Mental retardation in pediatric candidates for epilepsy surgery: The role of early seizure onset. Epilepsia. 2001;42(2):26874.

63. Ramantani G, Kadish NE, Strobl K, Brandt A, Stathi A, Mayer H, et al. Seizure and cognitive outcomes of epilepsy surgery in infancy and early childhood. Eur J Paediatr Neurol. 2013;17(5):498-506.

64. Baud MO, Perneger T, Rácz A, Pensel MC, Elger C, Rydenhag B, et al. European trends in epilepsy surgery. Neurology. 2018 Jul 10;91(2):e96-106.

65. Plewes DB, Kucharczyk W. Physics of MRI: a primer. J Magn Reson Imaging. 2012 May;35(5):1038-54.

66. Leach JL, Miles L, Henkel DM, Greiner HM, Kukreja MK, Holland KD, et al. Magnetic resonance imaging abnormalities in the resection region correlate with histopathological type, gliosis 
extent, and postoperative outcome in pediatric cortical dysplasia. J Neurosurg Pediatr. 2014 Jul 9;14(1):68-80.

67. Bien CG, Szinay M, Wagner J, Clusmann H, Becker AJ, Urbach H. Characteristics and Surgical Outcomes of Patients With Refractory Magnetic Resonance Imaging-Negative Epilepsies. Arch Neurol. 2009 Dec 1;66(12):1491-9.

68. Phi JH, Cho B-K, Wang K-C, Lee JY, Hwang YS, Kim KJ, et al. Longitudinal analyses of the surgical outcomes of pediatric epilepsy patients with focal cortical dysplasia. J Neurosurg Pediatr. 2010 Jul;6(1):49-56.

69. Mansouri A, Alhadid K, Valiante TA. Sudden unexpected death in epilepsy following resective epilepsy surgery in two patients withdrawn from anticonvulsants. J Clin Neurosci. 2015;22(9):15056.

70. Lerner JT, Salamon N, Hauptman JS, Velasco TR, Hemb M, Wu JY, et al. Assessment and surgical outcomes for mild type I and severe type II cortical dysplasia: A critical review and the UCLA experience. Epilepsia. 2009 Jun;50(6):1310-35.

71. Adler S, Lorio S, Jacques TS, Benova B, Gunny R, Cross JH, et al. Towards in vivo focal cortical dysplasia phenotyping using quantitative MRI. Neurolmage Clin [Internet]. 2017;15(January):95-105.

72. Kim DW, Kim S, Park S-H, Chung C-K, Lee SK. Comparison of MRI features and surgical outcome among the subtypes of focal cortical dysplasia. Seizure. 2012 Dec;21(10):789-94.

73. Colombo N, Tassi L, Deleo F, Citterio A, Bramerio M, Mai R, et al. Focal cortical dysplasia type lla and Ilb: MRI aspects in 118 cases proven by histopathology. Neuroradiology. 2012 Oct;54(10):1065-77.

74. Najm IM, Sarnat HB, Blümcke I. Review: The international consensus classification of Focal Cortical Dysplasia - a critical update 2018. Neuropathol Appl Neurobiol. 2018;44(1):18-31.

75. Xue H, Cai L, Dong S, Li Y. Clinical characteristics and post-surgical outcomes of focal cortical dysplasia subtypes. J Clin Neurosci. 2016 Jan;23:68-72.

76. Hauptman JS, Mathern GW. Surgical treatment of epilepsy associated with cortical dysplasia: 2012 update. Epilepsia. 2012 Sep;53 Suppl 4:98-104.

77. van Schooneveld MMJ, Braun KPJ, van Rijen PC, van Nieuwenhuizen O, Jennekens-Schinkel A. The spectrum of long-term cognitive and functional outcome after hemispherectomy in childhood. Eur J Paediatr Neurol. 2016;0(0):1-9.

78. Van Schooneveld MMJ, Braun KPJ. Cognitive outcome after epilepsy surgery in children. Brain Dev. 2013;35(8):721-9.

79. Lucas MM, Lenck-Santini PP, Holmes GL, Scott RC. Impaired cognition in rats with cortical dysplasia: Additional impact of early-life seizures. Brain. 2011;134(6):1684-93.

80. Vlooswijk MCG, Vaessen MJ, Jansen JFA, de Krom MCFTM, Majoie HJM, Hofman PAM, et al. Loss of network efficiency associated with cognitive decline in chronic epilepsy. Neurology. 2011 Sep 6;77(10):938-44.

81. Slinger G, Sinke MRT, Braun KPJ, Otte WM. White matter abnormalities at a regional and voxel level in focal and generalized epilepsy: A systematic review and meta-analysis. Neurolmage Clin. 2016;12:902-9. 
82. Rogawski MA, Löscher W. The neurobiology of antiepileptic drugs. Nat Rev Neurosci [Internet]. 2004 Jul;5(7):553-64.

83. Bourgeois BFD, Prensky AL, Palkes HS, Talent BK, Busch SG. Intelligence in epilepsy: A prospective study in children. Ann Neurol. 1983;14(4):438-44.

84. Hessen E, Lossius MI, Reinvang I, Gjerstad L. Influence of major antiepileptic drugs on attention, reaction time, and speed of information processing: Results from a randomized, double-blind, placebo-controlled withdrawal study of seizure-free epilepsy patients receiving monotherapy. Epilepsia. 2006;47(12):2038-45.

85. Knudsen El. Sensitive periods in the development of the brain and behavior. J Cogn Neurosci [Internet]. 2004 Oct;16(8):1412-25.

86. Boshuisen K, Van Schooneveld MMJ, Leijten FSS, De Kort GAP, Van Rijen PC, Gosselaar PH, et al. Contralateral MRI abnormalities affect seizure and cognitive outcome after hemispherectomy. Neurology. 2010;75(18):1623-30.

87. Watkins MW, Canivez GL. Temporal Stability of WISC - III Subtest Composite : Strengths and Weaknesses. Psychol Assess. 2004;16(2):133-8.

88. Caniyez GL, Watkins MW. Long-Term Stability of the Wechsler Intelligence Scale for Children-Third Edition. Psychol Assess. 1998;10(3):285-91.

89. Hanscombe KB, Trzaskowski M, Haworth CMA, Davis OSP, Dale PS, Plomin R. Socioeconomic status (SES) and children's intelligence (IQ): In a uk-representative sample SES moderates the environmental, not genetic, effect on IQ. PLoS One. 2012;7(2).

90. Pang T, Atefy R, Sheen V. Malformations of cortical development. Neurologist. 2008;14(3):181-91.

91. Téllez-Zenteno JF, Ronquillo LH, Moien-Afshari F, Wiebe S. Surgical outcomes in lesional and nonlesional epilepsy: A systematic review and meta-analysis. Epilepsy Res. 2010 May;89(2-3):310-8.

92. Wang ZI, Alexopoulos A V, Jones SE, Jaisani Z, Najm IM, Prayson R a. The pathology of magneticresonance-imaging-negative epilepsy. Mod Pathol. 2013 Aug 5;26(8):1051-8.

93. Knake S, Triantafyllou C, Wald LL, Wiggins G, Kirk GP, Larsson PG, et al. 3T phased array MRI improves the presurgical evaluation in focal epilepsies: A prospective study. Neurology. 2005 Oct 11;65(7):1026-31.

94. Zijlmans M, de Kort G a P, Witkamp TD, Huiskamp GM, Seppenwoolde J-H, van Huffelen AC, et al. 3T versus 1.5T phased-array MRI in the presurgical work-up of patients with partial epilepsy of uncertain focus. J Magn Reson Imaging. 2009 Aug;30(2):256-62.

95. Phal PM, Usmanov A, Nesbit GM, Anderson JC, Spencer D, Wang P, et al. Qualitative Comparison of 3-T and 1.5-T MRI in the Evaluation of Epilepsy. Am J Roentgenol. 2008 Sep;191(3):890-5.

96. Mellerio C, Labeyrie M-A, Chassoux F, Roca P, Alami O, Plat M, et al. 3T MRI improves the detection of transmantle sign in type 2 focal cortical dysplasia. Epilepsia. 2014 Jan 15;55(1):117-22.

97. van der Kolk AG, Hendrikse J, Zwanenburg JJM, Visser F, Luijten PR. Clinical applications of 7T MRI in the brain. Eur J Radiol. 2013 May;82(5):708-18.

98. Colon AJ, van Osch MJP, Buijs M, Grond J, Boon P, van Buchem MA, et al. Detection superiority of 7T MRI protocol in patients with epilepsy and suspected focal cortical dysplasia. Acta Neurol Belg. 2016;116(3):259-69. 
99. De Ciantis A, Barba C, Tassi L, Cosottini M, Tosetti M, Costagli M, et al. 7T MRI in focal epilepsy with unrevealing conventional field strength imaging. Epilepsia. 2016;57:445-454.

100. Mouthaan BE, Rados M, Barsi P, Boon P, Carmichael DW, Carrette E, et al. Current use of imaging and electromagnetic source localization procedures in epilepsy surgery centers across Europe. Epilepsia. 2016 May;57(5):770-6.

101. Teeuwisse WM, Brink WM, Webb AG. Quantitative assessment of the effects of high-permittivity pads in 7 Tesla MRI of the brain. Magn Reson Med. 2012 May;67(5):1285-93.

102. Madan N, Grant PE. New directions in clinical imaging of cortical dysplasias. Epilepsia. 2009 Oct;50 Suppl 9:9-18.

103. Knake S, Wehner T, Grant PE. Structural imaging of mesial temporal lobe epilepsy. In: Rosenow F., Ryvlin P, Lüders HO, editors. The Mesial Temporal Lobe Epilepsies. Montrouge, France: Editions John Libbey Eurotext; 2010. p. 165-73.

104. Blümcke I, Aronica E, Miyata H, Sarnat HB, Thom M, Roessler K, et al. International recommendation for a comprehensive neuropathologic workup of epilepsy surgery brain tissue: A consensus Task Force report from the ILAE Commission on Diagnostic Methods. Epilepsia. 2016 Mar;57(3):348-58.

105. Mellerio C, Roca P, Chassoux F, Danière F, Cachia A, Lion S, et al. The Power Button Sign: A Newly Described Central Sulcal Pattern on Surface Rendering MR Images of Type 2 Focal Cortical Dysplasia. Radiology. 2015 Feb;274(2):500-7.

106. Wellmer J, Quesada CM, Rothe L, Elger CE, Bien CG, Urbach H. Proposal for a magnetic resonance imaging protocol for the detection of epileptogenic lesions at early outpatient stages. Epilepsia. 2013 Nov;54(11):1977-87.

107. Blamire AM. The technology of MRI — the next 10 years? Br J Radiol. 2008 Aug;81(968):601-17.

108. Visser F, Zwanenburg JJM, Hoogduin JM, Luijten PR. High-resolution magnetization-prepared 3D-FLAIR imaging at 7.0 Tesla. Magn Reson Med. 2010 Jul;64(1):194-202.

109. Haacke EM, Mittal S, Wu Z, Neelavalli J, Cheng Y-CN. Susceptibility-weighted imaging: technical aspects and clinical applications, part 1. AJNR Am J Neuroradiol. 2009 Jan;30(1):19-30.

110. Mittal S, Wu Z, Neelavalli J, Haacke EM. Susceptibility-weighted imaging: technical aspects and clinical applications, part 2. AJNR Am J Neuroradiol. 2009 Feb;30(2):232-52.

111. Saini J, Kesavadas C, Thomas B, Kapilamoorthy TR, Gupta AK, Radhakrishnan A, et al. Susceptibility weighted imaging in the diagnostic evaluation of patients with intractable epilepsy. Epilepsia. 2009 Jun;50(6):1462-73.

112. Zwanenburg JJM, Versluis MJ, Luijten PR, Petridou N. Fast high resolution whole brain T2* weighted imaging using echo planar imaging at 7T. Neuroimage. 2011 Jun 15;56(4):1902-7.

113. Marín-Padilla M. The human brain intracerebral microvascular system: development and structure. Front Neuroanat. 2012 Jan;6(September):38.

114. la Fougère $C$, Rominger a, Förster $S$, Geisler J, Bartenstein P. PET and SPECT in epilepsy: a critical review. Epilepsy Behav. 2009 May;15(1):50-5.

115. Pendse N, Wissmeyer M, Altrichter S, Vargas $M$, Delavelle J, Viallon $M$, et al. Interictal arterial spin-labeling MRI perfusion in intractable epilepsy. J Neuroradiol. 2010 Mar;37(1):60-3. 
116. Logothetis NK, Pauls J, Augath M, Trinath T, Oeltermann a. Neurophysiological investigation of the basis of the fMRI signal. Nature. 2001 Jul 12;412(6843):150-7.

117. Veersema TJ, Swampillai B, Ferrier CH, Eijsden P, Gosselaar PH, van Rijen PC, et al. Long-term seizure outcome after epilepsy surgery in patients with mild malformation of cortical development and focal cortical dysplasia. Epilepsia Open. 2019 Dec 13;4(1):170-5.

118. Curatolo P, Moavero R, van Scheppingen J, Aronica E. mTOR dysregulation and tuberous sclerosisrelated epilepsy. Expert Rev Neurother. 2018;18(3):185-201.

119. Mühlebner A, Van Scheppingen J, Hulshof HM, Scholl T, lyer AM, Anink JJ, et al. Novel histopathological patterns in cortical tubers of epilepsy surgery patients with tuberous sclerosis complex. PLoS One. 2016;11(6):1-15.

120. Liang S, Zhang J, Yang Z, Zhang S, Cui Z, Cui J, et al. Long-term outcomes of epilepsy surgery in tuberous sclerosis complex. J Neurol. 2017 Jun;264(6):1146-54.

121. Veersema TJ, van Eijsden P, Gosselaar PH, Hendrikse J, Zwanenburg JJM, Spliet WGM, et al. 7 tesla T2*-weighted MRI as a tool to improve detection of focal cortical dysplasia. Epileptic Disord. 2016;18(3):315-23.

122. De Ciantis A, Barkovich AJ, Cosottini M, Barba C, Montanaro D, Costagli M, et al. Ultra-High-Field MR Imaging in Polymicrogyria and Epilepsy. Am J Neuroradiol. 2015 Feb 25;36(2):309-16.

123. Wintermark P, Lechpammer M, Warfield SK, Kosaras B, Takeoka M, Poduri A, et al. Perfusion Imaging of Focal Cortical Dysplasia Using Arterial Spin Labeling: Correlation With Histopathological Vascular Density. J Child Neurol. 2013 Nov 21;28(11):1474-82.

124. Blauwblomme T, Boddaert N, Chémaly N, Chiron C, Pages M, Varlet P, et al. Arterial Spin Labeling MRI: A step forward in non-invasive delineation of focal cortical dysplasia in children. Epilepsy Res. 2014 Dec;108(10):1932-9.

125. Pollock JM, Whitlow CT, Tan H, Kraft R a, Burdette JH, Maldjian J a. Pulsed arterial spin-labeled MR imaging evaluation of tuberous sclerosis. AJNR Am J Neuroradiol. 2009 Apr;30(4):815-20.

126. Northrup H, Krueger DA, International Tuberous Sclerosis Complex Consensus Group. Tuberous sclerosis complex diagnostic criteria update: recommendations of the 2012 linternational Tuberous Sclerosis Complex Consensus Conference. Pediatr Neurol. 2013 Oct;49(4):243-54.

127. Scholl T, Mühlebner A, Ricken G, Gruber V, Fabing A, Samueli S, et al. Impaired oligodendroglial turnover is associated with myelin pathology in focal cortical dysplasia and tuberous sclerosis complex. Brain Pathol. 2017;27(6):770-80.

128. Aronica E, Ravizza T, Zurolo E, Vezzani A. Astrocyte immune responses in epilepsy. Glia. 2012;60(8):1258-68.

129. Englot DJ, Berger MS, Barbaro NM, Chang EF. Factors associated with seizure freedom in the surgical resection of glioneuronal tumors. Epilepsia. 2012 Jan;53(1):51-7.

130. Prabowo AS, lyer AM, Anink JJ, Spliet WGM, van Rijen PC, Aronica E. Differential expression of major histocompatibility complex class I in developmental glioneuronal lesions. J Neuroinflammation. $2013 \operatorname{Jan} 24 ; 10(1): 12$.

131. Ishi K, Sasaki M, Kitagaki H, Sakamoto S, Yamaji S, Maeda K. Regional difference in cerebral blood flow and oxidative metabolism in human cortex. J Nucl Med. 1996;37(7):1086-8. 
132. Wong-Riley MTT. Energy metabolism of the visual system. Eye Brain [Internet]. 2010;2:99-116.

133. Young NA, Collins CE, Kaas JH. Cell and neuron densities in the primary motor cortex of primates. Front Neural Circuits. 2013;7(February):1-11.

134. Rockel AJ, Hiorns RW, Powell TPS. The Basic Uniformity in Structure of the Neocortex. Brain. 1980;103(2):221-44.

135. Skoglund TS, Pascher R, Berthold CH. Heterogeneity in the columnar number of neurons in different neocortical areas in the rat. Neurosci Lett. 1996;208(2):97-100.

136. Collins CE, Airey DC, Young NA, Leitch DB, Kaas JH. Neuron densities vary across and within cortical areas in primates. Proc Natl Acad Sci. 2010;107(36):15927-32.

137. Riddle DR, Sonntag WE, Lichtenwalner RJ. Microvascular plasticity in aging. Ageing Res Rev. 2003;2(2):149-68.

138. Mito T, Konomi H, Houdou S, Takashima S. Immunohistochemical study of the vasculature in the developing brain. Pediatr Neurol. 1991 Jan;7(1):18-22.

139. Coras R, de Boer OJ, Armstrong D, Becker A, Jacques TS, Miyata H, et al. Good interobserver and intraobserver agreement in the evaluation of the new ILAE classification of focal cortical dysplasias. Epilepsia. 2012 Aug;53(8):1341-8.

140. Bovetti S, Hsieh Y-C, Bovolin P, Perroteau I, Kazunori T, Puche AC. Blood vessels form a scaffold for neuroblast migration in the adult olfactory bulb. J Neurosci. 2007 May 30;27(22):5976-80.

141. Stubbs D, DeProto J, Nie K, Englund C, Mahmud I, Hevner R, et al. Neurovascular congruence during cerebral cortical development. Cereb Cortex. 2009 Jul;19 Suppl 1(SUPPL. 1):i32-41.

142. Farin A, Suzuki SO, Weiker M, Goldman JE, Bruce JN, Canoll P. Transplanted glioma cells migrate and proliferate on host brain vasculature: a dynamic analysis. Glia. 2006 Jun 1;53(8):799-808.

143. El-Hashemite $\mathrm{N}$, Walker V, Zhang H, Kwiatkowski DJ. Loss of Tsc1 or Tsc2 induces vascular endothelial growth factor production through mammalian target of rapamycin. Cancer Res. 2003;63(17):5173-7.

144. van Vliet EA, Aronica E, Gorter JA. Blood-brain barrier dysfunction, seizures and epilepsy. Semin Cell Dev Biol. 2015;38:26-34.

145. van Horssen J, Singh S, van der Pol S, Kipp M, Lim JL, Peferoen L, et al. Clusters of activated microglia in normal-appearing white matter show signs of innate immune activation. J Neuroinflammation. 2012 Jul 2;9(1):156.

146. Gorter JA, Van Vliet EA, Aronica E. Status epilepticus, blood-brain barrier disruption, inflammation, and epileptogenesis. Epilepsy Behav. 2015;49:13-6.

147. Rigau V, Morin M, Rousset M-C, de Bock F, Lebrun A, Coubes $P$, et al. Angiogenesis is associated with blood-brain barrier permeability in temporal lobe epilepsy. Brain. 2007 Jul;130(Pt 7):1942-56.

148. Alonso A, Reinz E, Fatar M, Hennerici MG, Meairs S. Clearance of albumin following ultrasoundinduced blood-brain barrier opening is mediated by glial but not neuronal cells. Brain Res. 2011;1411:9-16.

149. Ralay Ranaivo $H$, Wainwright MS. Albumin activates astrocytes and microglia through mitogenactivated protein kinase pathways. Brain Res. 2010 Feb 8;1313(1313C):222-31. 
150. Ahmad S, Hewett PW, Wang P, Al-Ani B, Cudmore M, Fujisawa T, et al. Direct evidence for endothelial vascular endothelial growth factor receptor-1 function in nitric oxide-mediated angiogenesis. Circ Res. 2006;99(7):715-22.

151. Papapetropoulos A, García-Cardeña G, Madri JA, Sessa WC. Nitric oxide production contributes to the angiogenic properties of vascular endothelial growth factor in human endothelial cells. J Clin Invest. 1997;100(12):3131-9.

152. Sun K, Cui J, Wang B, Jiang T, Chen Z, Cong F, et al. Magnetic resonance imaging of tuberous sclerosis complex with or without epilepsy at 7 T. Neuroradiology. 2018 Aug 5;60(8):785-94.

153. Ryvlin P, Cross JH, Rheims S. Epilepsy surgery in children and adults. Lancet Neurol. 2014;13(11):1114-26.

154. Bernasconi A, Cendes F, Theodore WH, Gill RS, Koepp MJ, Hogan RE, et al. Recommendations for the use of structural magnetic resonance imaging in the care of patients with epilepsy: $A$ consensus report from the International League Against Epilepsy Neuroimaging Task Force. Epilepsia. 2019;60(6):1054-68.

155. Veersema TJ, Ferrier CH, van Eijsden P, Gosselaar PH, Aronica E, Visser F, et al. Seven tesla MRI improves detection of focal cortical dysplasia in patients with refractory focal epilepsy. Epilepsia Open. 2017 Jun;2(2):162-71.

156. Colon AJ, Osch MJP van, Buijs M, Grond J v. d., Hillebrand A, Schijns O, et al. MEG-guided analysis of 7T-MRI in patients with epilepsy. Seizure. 2018;60(May):29-38.

157. Trattnig S, Springer E, Bogner W, Hangel G, Strasser B, Dymerska B, et al. Key clinical benefits of neuroimaging at 7 T. Neuroimage. 2018;168(November 2016):477-89.

158. Zucca I, Milesi G, Medici V, Tassi L, Didato G, Cardinale F, et al. Type II focal cortical dysplasia: Ex vivo 7T magnetic resonance imaging abnormalities and histopathological comparisons. Ann Neurol. 2016;79(1):42-58.

159. Reeves C, Tachrount M, Thomas D, Michalak Z, Liu J, Ellis M, et al. Combined Ex Vivo 9.4T MRI and Quantitative Histopathological Study in Normal and Pathological Neocortical Resections in Focal Epilepsy. Brain Pathol. 2016;26(3):319-33.

160. Garbelli R, Zucca I, Milesi G, Mastropietro a, D'Incerti L, Tassi L, et al. Combined 7-T MRI and histopathologic study of normal and dysplastic samples from patients with TLE. Neurology. 2011 Mar 29;76(13):1177-85.

161. Goubran M, de Ribaupierre S, Hammond RR, Currie C, Burneo JG, Parrent AG, et al. Registration of in-vivo to ex-vivo MRI of surgically resected specimens: A pipeline for histology to in-vivo registration. J Neurosci Methods. 2015;241:53-65.

162. Zucca I, Milesi G, Padelli F, Rossini L, Gozzo F, Figini M, et al. An image registration protocol to integrate electrophysiology, MRI and neuropathology data in epileptic patients explored with intracerebral electrodes. J Neurosci Methods. 2018;303:159-68.

163. Dauguet J, Delzescaux T, Condé F, Mangin JF, Ayache N, Hantraye P, et al. Three-dimensional reconstruction of stained histological slices and 3D non-linear registration with in-vivo MRI for whole baboon brain. J Neurosci Methods. 2007;164(1):191-204. 
164. Pfefferbaum A, Sullivan E V., Adalsteinsson E, Garrick T, Harper C. Postmortem MR imaging of formalin-fixed human brain. Neuroimage. 2004;21(4):1585-95.

165. Noordmans HJ, Biesen P, Roode R, Verdaasdonk R. Software helps see the unseen in ophthalmology. Laser Focus World. 2008 May 1;44.

166. Noordmans HJ, van Blooijs D, Siero JCW, Zwanenburg JJM, Klaessens JHGM, Ramsey NF. Detailed view on slow sinusoidal, hemodynamic oscillations on the human brain cortex by Fourier transforming oxy/deoxy hyperspectral images. Hum Brain Mapp. 2018;39(9):3558-73.

167. Louis D, Ohgaki H, Wiestler OD, Cavenee W. World Health Organization Histological Classification of Tumours of the Central Nervous System. 2016.

168. Simpson SL, Prayson R a. Post-surgical outcome for epilepsy associated with type I focal cortical dysplasia subtypes. Mod Pathol. 2014 Apr 18;1-6.

169. Tassi L, Garbelli R, Colombo N, Bramerio M, Russo G Lo, Mai R, et al. Electrolcinical, MRI and surgical outcomes in 100 epileptic patients with type II FCD. Epileptic Disord. 2012;14(3):257-66.

170. Noli D, Bartuluchi M, González FS, Kaltenmeier MC, Cersosimo R, Rugilo C, et al. Type II focal cortical dysplasia: electroclinical study and surgical outcome in 31 pediatric patients. Childs Nerv Syst. 2013 Jul 7;

171. Yao K, Mei X, Liu X, Duan Z, Liu C, Bian Y, et al. Clinical characteristics, pathological features and surgical outcomes of focal cortical dysplasia (FCD) type II: Correlation with pathological subtypes. Neurol Sci. 2014;35(10):1519-26.

172. Chen J, Huang Z, Li L, Ren L, Wang Y. Histological type of focal cortical dysplasia is associated with the risk of postsurgical seizure in children and adolescents. Ther Clin Risk Manag. 2019;15:877-84.

173. Lamberink HJ, Otte WM, Blumcke I, Braun KPJ. Seizure outcome and use of antiepileptic drugs after epilepsy surgery according to histopathological diagnosis: a retrospective cohort study. Lancet Neurol. 2020;

174. Baldassari S, Ribierre T, Marsan E, Adle-Biassette H, Ferrand-Sorbets S, Bulteau C, et al. Dissecting the genetic basis of focal cortical dysplasia: a large cohort study. Acta Neuropathol. 2019;138(6):885900.

175. Budinger TF, Bird MD. MRI and MRS of the human brain at magnetic fields of $14 \mathrm{~T}$ to $20 \mathrm{~T}$ : Technical feasibility, safety, and neuroscience horizons. Neuroimage. 2018;168(February 2017):509-31.

176. Mooiweer R, Sbrizzi A, Raaijmakers AJE, van den Berg CAT, Luijten PR, Hoogduin H. Combining a reduced field of excitation with SENSE-based parallel imaging for maximum imaging efficiency. Magn Reson Med. 2016;00:1-9.

177. Kim KN, Hernandez D, Seo JH, Noh Y, Han Y, Ryu YC, et al. Quantitative assessment of phased array coils with different numbers of receiving channels in terms of signal-to-noise ratio and spatial noise variation in magnetic resonance imaging. PLoS One. 2019;14(7):1-13.

178. Feldman RE, Delman BN PP, Dyvorne H, Rutland JW, Yoo J et a. 7T study of patients with previously normal MRI exams. Plosone. 2019;14(3):1-24.

179. Hu W, Wang X, Liu L, Shao X, Zhang K, Ma Y. Multimodality Image Post-processing in Detection of Extratemporal MRI-Negative Cortical Dysplasia. 2018;9(June):1-9. 
180. Wong-Kisiel LC, Tovar Quiroga DF, Kenney-Jung DL, Witte RJ, Santana-Almansa A, Worrell GA, et al. Morphometric analysis on T1-weighted MRI complements visual MRI review in focal cortical dysplasia. Epilepsy Res. 2018;140(January):184-91.

181. Wang W, Lin Y, Wang S, Jones S, Prayson R, Moosa ANV, et al. Voxel-based morphometric magnetic resonance imaging postprocessing in non-lesional pediatric epilepsy patients using pediatric normal databases. Eur J Neurol. 2019;26(7):969-e71.

182. Jin B, Krishnan B, Adler S, Wagstyl K, Hu W, Jones S, et al. Automated detection of focal cortical dysplasia type II with surface-based magnetic resonance imaging postprocessing and machine learning. Epilepsia. 2018;(February):1-11.

183. Wang ZI, Jones SE, Jaisani Z, Najm IM, Prayson R a, Burgess RC, et al. Voxel-based morphometric magnetic resonance imaging (MRI) postprocessing in MRI-negative epilepsies. Ann Neurol. 2015 Mar 25;1-16.

184. Hong S-J, Kim H, Schrader D, Bernasconi N, Bernhardt BC, Bernasconi A. Automated detection of cortical dysplasia type II in MRI-negative epilepsy. Neurology. 2014 Jun 4;1-8.

185. Wagner J, Weber B, Urbach H, Elger CE, Huppertz H-J. Morphometric MRI analysis improves detection of focal cortical dysplasia type II. Brain. 2011 Oct;134(Pt 10):2844-54.

186. Martin P, Winston GP, Bartlett P, de Tisi J, Duncan JS, Focke NK. Voxel-based magnetic resonance image postprocessing in epilepsy. Epilepsia. 2017;58(9):1653-64.

187. El Tahry R, Santos SF, Vrielynck P, de Tourtchaninoff M, Duprez T, Vaz GR, et al. Additional clinical value of voxel-based morphometric MRI post-processing for MRI-negative epilepsies: a prospective study. Epileptic Disord. 2020;22(2):156-64.

188. Karamat MI, Darvish-Molla S, Santos-Diaz A. Opportunities and Challenges of 7 Tesla Magnetic Resonance Imaging: A Review. Crit Rev Biomed Eng. 2016;44(1-02):73-89.

189. Godenschweger F, Kägebein U, Stucht D, Yarach U, Sciarra A, Yakupov R, et al. Motion correction in MRI of the brain. Phys Med Biol. 2016 Mar 7;61(5):R32-56.

190. Pittau F, Ferri L, Fahoum F, Dubeau F, Gotman J. Contributions of EEG-fMRI to assessing the epileptogenicity of focal cortical dysplasia. Front Comput Neurosci. 2017;11(February).

191. Pan JW, Kuzniecky RI. Utility of magnetic resonance spectroscopic imaging for human epilepsy. Quant Imaging Med Surg. 2015;5(2):313-31322.

192. Pan JW, Duckrow RB, Gerrard J, Ong C, Hirsch LJ, Resor SR, et al. 7T MR spectroscopic imaging in the localization of surgical epilepsy. Epilepsia. 2013 Sep;54(9):1668-78. 


\section{Nederlandse samenvatting}

\section{Achtergrond}

Een epileptische aanval wordt gedefinieerd als het tijdelijk optreden van symptomen ten gevolge van abnormale, overmatige of gelijktijdige activiteit van neuronen in de hersenen. Een epileptische aanval kan verschillende vormen aannemen. Dit is mede afhankelijk van de locatie in de hersenen waar de aanval begint en hoe de activiteit zich verspreid naar andere delen van de hersenen. Er kunnen veranderingen optreden in waarneming, bewegen, gedrag, denken en autonome functies. Er is sprake van de aandoening 'epilepsie' wanneer er een blijvende neiging is om epileptische aanvallen te ontwikkelen zonder aanwijsbare externe uitlokkende factor (zoals medicatie, drugs, hersenschudding etc.). Epilepsie kan focaal zijn, waarbij de aanvallen in één of meerdere afgebakende gebieden ontstaan, of gegeneraliseerd, waarbij aanvallen in het gehele brein ontstaan. Bij de meeste patiënten met epilepsie wordt in de eerste plaatst gestart met medicatie, desondanks houdt tot ongeveer één derde van de patiënten epileptische aanvallen. Deze patiënten hebben medicatie-resistente epilepsie ofwel refractaire epilepsie.

Dit proefschrift gaat over patiënten met epilepsie ten gevolge van zogenaamde Focale Corticale Dysplasie, afgekort als FCD. Dit is één van de meest voorkomende oorzaken van focale epilepsie. De grote hersenen zijn opgebouwd uit een diepere laag witte stof waarin alle lange zenuwbanen lopen met daaroverheen als buitenste laag de hersenschors. De hersenschors (ook wel grijze stof of cortex) bestaat uit zes te onderscheiden lagen van zenuwcellen (neuronen). Per laag verschillen de neuronen van vorm en in functie. Bij FCD is de opbouw van deze lagen verstoord en kunnen er ook afwijkende neuronen voorkomen. In dit gedeelte van de hersenen is de functie verstoord, waardoor er makkelijk epileptische aanvallen kunnen ontstaan.

In 2011 is er een nieuw systeem opgesteld door de International League Against Epilepsy (ILAE) om de verschillende soorten FCD te classificeren op basis van het histologisch (microscopie) onderzoek. Het betreft de volgende classificaties:

FCD Type I: alleen afwijkende gelaagdheid van de hersenschors. Te onderscheiden in subtype la met gestoorde radiaire gelaagdheid en lb met gestoorde tangentiele (horizontale) gelaagdheid. In Ic is er een combinatie van gestoorde radiaire én tangentiele gelaagdheid FCD Type II: afwijkende gelaagdheid van de hersenschors én afwijkende neuronen. Bij Ila zijn er afwijkende, onrijpe neuronen. In type Ilb zijn er typische balloncellen: grote ongedifferentieerde hersencellen. 
FCD Type III: FCD in combinatie met andere hersenafwijkingen: hippocampale sclerose, tumoren, vasculaire malformaties of afwijkingen ten gevolge van hersenschade vroeg in het leven (bijvoorbeeld littekens na een bloeding of infectie rond de geboorte). Hippocampale sclerose is op zichzelf een van de meest voorkomende diagnoses bij patiënten met epilepsie. Dit wordt gekenmerkt door verlies van neuronen en verlittekening van de hippocampus (dit is een gepaarde structuur aan de binnenzijde van de temporaalkwabben met een belangrijke rol in het geheugen)

Er is ook een variant genaamd mild Malformation of Cortical Development (mMCD). Hierbij is de cortex normaal maar zijn er abnormaal veel neuronen in de meest oppervlakkige cortexlaag (mMCD type 1) en/of de witte stof (mMCD type 2). mMCD wordt gezien als de mildste vorm van dysplasie in het FCD-spectrum wat betreft de histologische afwijkingen. Zie ook figuur 1 voor voorbeelden van histologische kenmerken van mMCD en FCD.

We weten nog niet precies waarom deze dysplasieën ontstaan, maar er zijn steeds meer aanwijzingen dat er fouten ontstaan in bepaalde fasen van de hersenontwikkeling. Hierbij zijn meerdere eiwitten betrokken die door genetische afwijkingen niet normaal functioneren.

Patiënten met FCD hebben doorgaans al voor het $10^{\mathrm{e}}$ levensjaar een eerste aanval. Deze aanvallen zijn bij veel patiënten frequent en niet goed te behandelen met medicatie. Patiënten ervaren vaak ook andere problemen die verband hebben met de onderliggende hersenafwijking, de epileptische aanvallen of van de anti-epileptische medicatie. Voorbeelden hiervan zijn migraine, vaatlijden en traumatische verwondingen. Patiënten met focale epilepsie hebben een hoger risico op psychiatrische problemen zoals stemmings-, angst-, en persoonlijkheidsstoornissen en psychoses. Verder hebben meer dan de helft van de jonge patiënten met FCD cognitieve problemen, gedefinieerd als een IQ-score lager dan 70. Dit is het gevolg van de lokaal verstoorde architectuur en daardoor ook verstoorde functie van de hersenen, maar ook door een ontregeling van het globaal functioneren van de hersenen door het optreden van de epileptische aanvallen. Bovendien heeft anti-epileptische medicatie een negatief effect doordat ook de normale functies van de hersenen worden beïnvloed.

Voor patiënten met medicatie-resistente epilepsie kan chirurgische behandeling een optie zijn, met vaak goede kansen op vermindering van epileptische aanvallen tot zelfs volledige aanvalsvrijheid. Het mogelijke vooruitzicht om te kunnen stoppen met de medicatie is een ander argument voor opereren, in verband met de eerdergenoemde bijwerkingen en negatieve effecten op de cognitie.

Afhankelijk van het ziektebeeld en de hersenafwijkingen wordt het afwijkend stuk hersenen of een volledige hersenkwab verwijderd. Soms wordt een gebied met afwijkende functie losgekoppeld van de rest van de hersenen, zodat de epileptische aanvallen zich niet verder kunnen verspreiden naar de rest van de hersenen. 

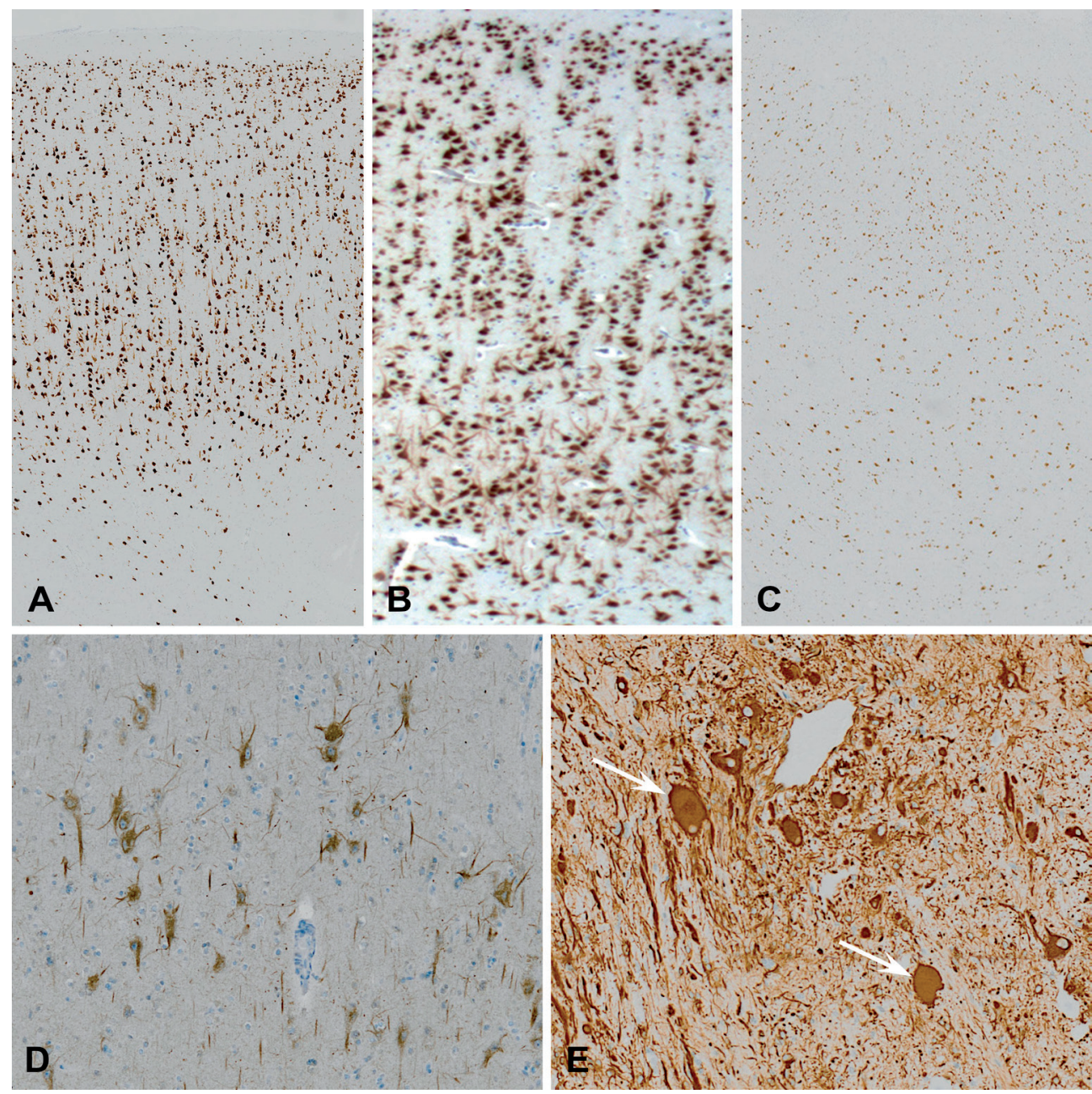

Figuur 1. Voorbeelden van histologische kenmerken van mMCD en FCD.

A: $\mathrm{mMCD}$ type 2 . Normale opbouw van de cortex, overmaat van neuronen in moleculaire laag (boven in figuur) en witte stof (onder in figuur) (NeuN kleuring).

B: FCD la. Abnormale radiaire gelaagdheid, met typische columnaire organisatie (NeuN kleuring). C: FCD Ilb. In FCD II subtypes is er vaak volledige verstoring van de corticale gelaagdheid en verlies van de grens tussen grijze en witte stof.

D: FCD Ila. Immature, dysmorfe neuronen met abnormale oriëntatie en positieve kleuring door SMI32, kenmerkend voor FCD Ila (SMI32 kleuring).

E: FCD Ilb. Typische balloncellen, grote ongedifferentieerde cellen zonder Nissl-substantie en met positieve aankleuring voor vimentine (vimentine kleuring). 
Eerder onderzoek heeft laten zien dat de helft van de geopereerde patiënten met FCD geen enkele epileptische aanval meer heeft. De belangrijkste voorspeller van aanvalsvrijheid is compleetheid van de resectie, dus of het afwijkende weefsel volledig is weggehaald. Andere belangrijke voorspellers hebben te maken met factoren die complete resectie vergemakkelijken, zoals een afwijking die zichtbaar is op de MRI, een afwijking in de temporaalkwab en afwijkingen die zich slechts in één kwab bevinden. Ook een kortere epilepsieduur en chirurgie op kinderleeftijd geven een hogere kans op aanvalsvrijheid. Er is echter nog relatief weinig bekend over de relatie tussen kans op aanvalsvrijheid en het type FCD, al zijn er wel enkele studies uitgevoerd die betere resultaten vonden voor FCD type II.

De meeste studies laten een verbetering zien in cognitief functioneren na epilepsiechirurgie wat samenhangt met aanvalsvrijheid, opleidingsniveau van de ouders, aanvalsfrequentie voor de operatie, de onderliggende oorzaak van epilepsie en anti-epileptische medicatie. Kinderen met een vroeg begin van epilepsie en reeds langer bestaande epilepsie hebben ernstigere cognitieve problemen. Er zijn nog niet verschillen gerapporteerd in cognitieve vermogens tussen de verschillende histologische mMCD/FCD types.

De laatste jaren is chirurgie een steeds belangrijkere behandeling van epilepsie geworden. Dit is het gevolg van de goede uitkomsten van operatieve behandeling en een lage kans op complicaties, en de grote technische ontwikkeling. Op dit moment is chirurgie de enige kans op aanvalsvrijheid bij medicatie-resistente epilepsie. Nauwkeurige lokalisatie van een afwijking is essentieel voor succesvolle chirurgie. Er gaat hier dan ook een uitgebreid diagnostisch traject vooraf. In eerste plaats wordt een elektro-encefalografie (EEG) en een MRI-scan gemaakt. Het EEG registreert hersenactiviteit met elektrodes op de hoofdhuid. Specifieke patronen tijdens en tussen aanvallen geven de locatie aan waar de epileptische activiteit ontstaat. De MRI (magnetic resonance imaging), ook wel magneetscan genoemd, levert beelden van de anatomie van de hersenen. Dit is mogelijk door magnetische excitatie van atoomkernen en verschillen in de relaxatie tussen weefsels te meten. Atoomkernen gedragen zich door de sterke magneet als kleine tolletjes. Door elektromagnetische golven van de MRI-scanner worden deze tolletjes in een bepaalde richting gedwongen (de excitatie). De gelijke bewegingen van atoomkernen zorgen voor een meetbaar signaal. Vervolgens gaan de atoomkernen weer terug naar de neutrale stand en verdwijnt de synchroniciteit van de bewegingen en daarmee het signaal (relaxatie). De MRI is zeer veelzijdig doordat verschillende soorten opnamen kunnen worden gebruikt, die weer specifieke weefseleigenschappen uitvergroten. Deze verschillende soorten opnamen worden sequenties genoemd, zoals $T_{1}$ en $T_{2}$ gewogen sequenties (niet te verwarren met tesla of T als maat van magneetveldsterkte). Naast anatomische informatie is MRI ook in staat informatie te geven over activiteit van de hersenen op basis van het zuurstofgehalte in hersenen en bloedvaten. Bloed heeft meetbare verschillen in MRI-signaal afhankelijk van 
hoeveel zuurstof het bevat. Dit is het zogenaamde Blood Oxygen Level Dependent (BOLD) effect wat wordt benut in functionele MRI (fMRI). fMRI wordt ook gebruikt om aan te tonen waar bijvoorbeeld taalfuncties zitten, wat weer invloed heeft op de gekozen behandeling.

Naast het EEG en de MRI worden nog verschillende andere technieken ingezet, zoals magneto-encefalografie (MEG). Dit is een zeer gevoelig apparaat wat subtiele magnetische velden meet die ontstaan door de actiepotentialen in de hersenen. De nucleair geneeskundige onderzoeken Positronemissietomografie (PET) en Single Photon Emission Computed Tomography (SPECT) geven informatie over hoe actief regio's binnen de hersenen zijn en daarmee ook of er sprake kan zijn van epileptische activiteit. Er wordt een licht radioactieve stof in de bloedbaan gespoten, welke afhankelijk van het gekozen middel verzamelt in bepaalde weefsels en daar radioactief verval ondergaat, wat de scanner vervolgens registreert. Als beeldvorming en de niet-invasieve EEG-onderzoeken geen zekere locatie van de bron van de epileptische aanvallen heeft kunnen aantonen, dan bestaat nog de optie om een invasief EEG te maken. Hierbij wordt de patiënt geopereerd om de elektrodes als een matje tussen de hersenen en schedel te plaatsen, of als naalden in de hersenen. Doordat er een veel kleinere afstand is tussen de elektrode en de neuronen is het invasieve EEG gevoeliger voor het registeren van lokale afwijkende hersenactiviteit.

De MRI speelt een steeds grotere rol in het preoperatieve traject. Een structurele afwijking is een sterke en betrouwbare aanwijzing voor de aanwezigheid van een bron van de epilepsie. Echter is er niet altijd een exacte overeenkomst tussen het afwijkend gebied op de MRI en het weefsel dat de epileptische aanvallen veroorzaakt. Toch maakt een duidelijke en goed begrensde afwijking aanvullende tests vaak onnodig en vormt het een goed doelwit voor een operatie of invasief EEG-onderzoek. Desondanks kan ook zonder afwijking op MRI een operatie plaatsvinden op basis van andere onderzoeken, maar de kans op aanvalsvrijheid wordt daarmee kleiner. Het is bekend dat bij $15-40 \%$ van de patiënten die geopereerd zijn de FCD niet zichtbaar was op MRI. Voor mMCD kan dit zelfs meer dan $50 \%$ zijn.

Er zijn een aantal typische kenmerken van mMCD en FCD op de MRI. Zo is er een onscherpe grens tussen de hersenschors en onderliggende witte stof en zijn er lokale signaalveranderingen. Ook kan de hersenschors verdikt zijn of andere vormen van de windingen hebben. FCD type Il wordt gekenmerkt door het zogenaamde 'transmantle sign', een wigvormige band van signaalveranderingen richting het midden van de hersenen. De overige types laten zich minder makkelijk onderscheiden op basis van MRI. Zie ook figuur 2 voor voorbeelden van MRI-afwijkingen bij FCD.

Idealiter levert de MRI precies de informatie aan het behandelteam wat voor soort afwijking het betreft en wat de grenzen zijn met normaal hersenweefsel en wat voor soort afwijking het betreft. 

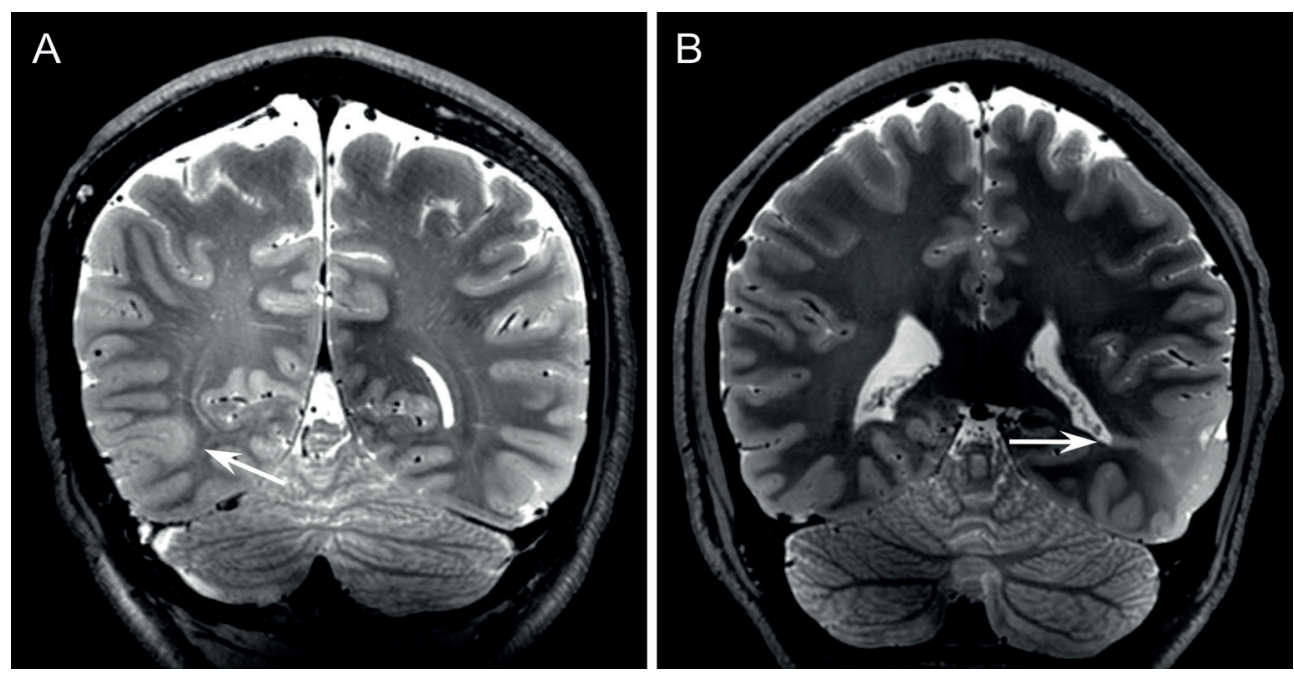

Figuur 2. Typische MRI-bevindingen bij FCD. Coronale reconstructies van 3D $T_{2}$ beelden op 7T MRI. Twee patiënten met FCD Ilb.

A: Dysplasie in de bodem van groeve rechts met verdikking van de cortex, vervanging van de grijswitte stof overgang en signaalveranderingen in cortex en witte stof.

B: Uitgesproken FCD laesie achter in de temporaalkwab met ook typische 'transmantle sign'.

Ondanks de grote ontwikkelingen van de laatste decennia in MRI en beeldverwerkingstechnieken, is het te verwachten dat verdere ontwikkelingen een belangrijke verbetering kunnen brengen in de chirurgische behandeling van epilepsie. In de afgelopen tien jaar zijn 7 tesla (T) MRI-systemen voor menselijk gebruik beschikbaar gekomen. Zeven tesla staat voor de sterkte van de magneet, en in vergelijking met de huidige standaard $-1,5$ en 3 tesla MRI-zorgt de sterkere magneet voor een betere signaal-ruisverhouding. Deze betere signaal-ruisverhouding kan worden gebruikt om beelden met hogere resolutie en meer contrast te maken. Daar tegenover staat dat bij een sterkere magneet er grotere technische uitdagingen zijn om verstoringen in het beeld te onderdrukken. Ook is het 7T MRI-systeem vele malen duurder. Daarom is het noodzakelijk om doormiddel van wetenschappelijk onderzoek aan te tonen dat de theoretische voordelen van 7T MRI ook werkelijk leiden tot betere uitkomsten.

Sinds het begin van de epilepsiechirurgie in het eind van de $19^{\mathrm{e}}$ eeuw hebben er grote veranderingen plaatsgevonden in de zorg voor mensen met medicatie-resistente epilepsie. Ons begrip over hoe epileptische aanvallen ontstaan, de relaties met structurele hersenafwijkingen en de functionele anatomie is sterk toegenomen. Chirurgische technieken zijn verfijnd en nieuwe methodes zijn ontwikkeld. Patiënten met aanlegstoornissen als MCD/FCD maken een steeds groter deel uit van de totale patiëntpopulatie en er gaat veel onderzoek uit naar uit om de zorg verder te verbeteren. Er blijven belangrijke uitdagingen: onder andere het vaststellen van de oorzaak van de epilepsie, met name 
bij afwezigheid van grove structurele afwijkingen en de identificatie van de grens tussen epileptogeen en normaal weefsel, te meer omdat verschillende modaliteiten vaak niet perfect overeenkomende informatie geven. De keuze voor de beste behandeling vereist een sterke a priori diagnose, een goed begrip van de kans van slagen en van de risico's van de verschillende behandelopties.

Doel van het onderzoek beschreven in dit proefschrift was om meer begrip te krijgen over hoe bij mMCD/FCD histologische verschillen gerelateerd zijn aan patiënteigenschappen en aan de kans op een succesvolle chirurgische behandeling. Bovendien werd onderzocht hoe de diagnostische beeldvorming kan worden verbeterd met betrekking tot subtiele afwijkingen die met huidige technieken niet worden gezien. Meer accurate identificatie van de aanwezigheid en begrenzing van $\mathrm{mMCD}$ en FCD-laesies zal er uiteindelijk voor zorgen dat meer patiënten kunnen profiteren van chirurgische behandelingen met betere uitkomsten.

\section{Bevindingen in dit proefschrift}

De eerste studie evalueerde de histopathologische diagnoses en klinische kenmerken in eerder geopereerde patiënten. We hebben retrospectief een groep bestudeerd van alle patiënten geopereerd in het UMC Utrecht in de periode 2000-2012 met een histologische diagnose passend bij mMCD of FCD. Voor deze studie hebben we de histologische classificaties gereviseerd volgens de 2011 ILAE richtlijnen. In hoofdstuk 2 beschrijven we dit cohort van 88 patiënten. 9\% (8/88) had een FCD I diagnose en 56\% (49/88) FCD II. Het grote aantal met $\mathrm{mMCD}(31 / 88,35 \%)$ in vergelijking met voorgaande studies is opvallend. FCD Il kwam relatief vaker voor in de frontaalkwab, mMCD en FCD I vaker in de temporaalkwab. Patiënten met mMCD hadden een later epilepsiedebuut en ondergingen ook op latere leeftijd chirurgie in vergelijking met patiënten met FCD. In de totale groep leidde epilepsiechirurgie tot langdurige aanvalsvrijheid bij 49\%, bij een mediane follow-up duur van 7 jaar; $36 \%$ was ook volledig gestopt met anti-epileptische medicatie. Patiënten met FCD Ilb werden het vaakst aanvalsvrij, bij mMCD was de kans op aanvalsvrijheid juist kleiner.

We bestudeerden een groep van 42 kinderen, die onderdeel waren van de hierboven beschreven groep, wat betreft hun cognitieve vaardigheden voor en na epilepsiechirurgie. Deze bevindingen zijn beschreven in hoofdstuk 3. Van deze groep kinderen had 51\% een cognitieve beperking, gedefinieerd als een intelligentiequotiënt (IQ) lager dan 70 . Preoperatief cognitief functioneren was lager in patiënten met een vroeg epilepsiedebuut. $\mathrm{Er}$ is sprake van een ernstige cognitieve beperking bij een IQ of ontwikkelingsindex $(\mathrm{O}$, ontwikkelingsniveau in jaren gedeeld door kalenderleeftijd) onder de 55. Patiënten met een ernstige cognitieve beperking worden vaak geopereerd op zeer jonge leeftijd vanwege optreden van epileptische encefalopathie, wat leidde tot een schijnbare correlatie tussen 
laag cognitief functioneren en operatie op jonge leeftijd en daarbij korte epilepsieduur. In de patiënten met preoperatief IQ/OI >55 was een korte epilepsieduur geassocieerd met betere cognitief functioneren. Alle kinderen met FCD I hadden een cognitieve beperking, en het gemiddeld IQ/ontwikkelingsindex was significant lager dan bij kinderen met mMCD of FCD type II. Na chirurgie was er bij $43 \%$ klinisch relevante verbetering in de cognitie, gedefinieerd als $\geq 7$ punt stijging in IQ/OI. Bij $46 \%$ was cognitief functioneren stabiel en bij $11 \%$ was er verslechtering van $7 \mathrm{IQ} / \mathrm{OI}$ punten of meer. Alleen een diagnose van epileptische encefalopathie voorspelde een cognitieve verbetering na chirurgie. We vonden geen associatie tussen postoperatieve cognitieve uitkomst en aanvalsvrijheid.

In hoofdstuk 4 onderzochten we een groep van 40 patiënten bij wie er een aanvullende 7T MRI was verricht in het preoperatieve diagnostische traject. Deze 7T MRI was geïndiceerd voor patiënten met medicatie-resistente epilepsie en veronderstelde focale epilepsie, zonder dat er een afwijking was aangetoond op één of meerdere MRI-scans op lagere veldsterkte. Deze eerdere MRI moest wel van adequate kwaliteit zijn en gemaakt met epilepsie-specifieke protocollen. Ook werd een 7T MRI gemaakt wanneer een verborgen afwijking werd verdacht in combinatie met MRI-zichtbare hippocampale sclerose. Bij multidisciplinaire beoordeling van het 7T MRI-onderzoek werden nieuwe afwijkingen geïdentificeerd bij negen patiënten (23\%), wat invloed had op verdere medische besluitvorming. Ten tijde van de studie waren zes van deze patiënten reeds geopereerd, waarbij er bevestiging was van FCD ILAE type IIA $(n=2)$, FCD IIB $(n=1), m M C D$ type II $(n=2)$ en mMCD met oligodendrogliale hyperplasie (MOGHE) $(n=1)$.

Gedurende de herevaluatie van de klinisch geïndiceerde 7T MRI-scans ontdekten we een patroon wat samenviel met histologisch bevestigde mMCD/FCD laesies van specifieke signaalveranderingen op de $T_{2}{ }^{*}$ sequentie. Deze $T_{2}{ }^{*}$ sequentie is zeer gevoelig voor de aanwezigheid van onder andere bloedafbraakproducten, kalk en ijzerdeposities. Onze observaties in een groep van zes patiënten hebben we beschreven in hoofdstuk $\mathbf{5}$. In vier van de zes patiënten, waren er gebieden met hypointensiteiten (laag signaal) met een vertakkende, deels tortueuze configuratie, en een signaal passend bij veneus bloed, suggestief voor toegenomen veneuze vaatstructuren in de groeven aangrenzend aan afwijkende hersenschors, en in mindere mate ook in het hersenparenchym. In twee van deze patiënten waren er geen afwijkingen gezien op eerdere 3T MRI en bij één patiënt liet ook de 7T MRI geen structurele afwijking zien. We vergeleken de beelden vervolgens met 7T MRI-beelden van acht gezonde proefpersonen, waarbij symmetrische veneuze vasculaire structuren in de hersenvliezen duidelijk zichtbaar waren, maar het hierboven beschreven fenomeen niet werd gezien.

Vervolgens onderzochten we in de studie in hoofdstuk 6 de hypothese dat de MRIbevindingen beschreven in hoofdstuk 5 betekenen dat er een relatie is tussen de 
dichtheid van microvasculatuur en corticale dysplasie. We voerden een kwantitatieve immunohistochemische analyse uit, van microvasculaire densiteit en ontsteking in hersenweefsel van 97 patiënten met mMCD, FCD of tubereuze sclerose complex (TSC) en van 14 personen zonder epilepsie, gematched op leeftijd en lokalisatie in het brein als controlegroep.

TSC is een genetische aandoening waarbij er hamartomen (goedaardige gezwellen) kunnen ontstaan in verschillende organen, onder andere in hersenen, nieren, hart, lever, ogen en huid. In de hersenen worden deze hamartomen tubers genoemd, en is er microscopische grote gelijkenis met FCD Ilb. Patiënten met TSC kunnen ook epilepsie en cognitieve problemen hebben en klachten gerelateerd aan de orgaanafwijkingen.

We vonden een hogere vaatdichtheid in de hersenschors en witte stof in FCD en tubers ten opzichte van het omliggende weefsel, onafhankelijk van de leeftijd. In tubers, en in mindere mate ook bij FCD, was er een toename in vaatdichtheid in de hersenshors op hogere leeftijd, wat niet het geval was in de controlegroep. In vergelijking met weefsel van de controlegroep was er in de tubers bij TSC een verhoogde vaatdichtheid in de witte stof, die geassocieerd kon worden met activatie van microglia en een toename van ontstekingscellen. Deze relatie werd niet gezien in de hersenschors. Er was geen significant verschil in vaatdichtheid tussen FCD en de controlegroep zonder epilepsie.

De eerste klinische ervaringen met 7T MRI bij patiënten met epilepsie zijn veelbelovend, hoewel het ook duidelijk werd dat in de meerderheid van de patiënten met een normale 3T MRI, ook de 7T MRI geen afwijking laat zien. Eerder werd al benadrukt wat het belang is van preoperatieve identificatie en bepaling van de begrenzing van een afwijking en van een accurate histopathologische diagnose. Verdere vooruitgang en aanvullende kennis kan worden verworven door analysehulpmiddelen die het mogelijk maken de 7T MRI-beelden met histologische bevindingen te associëren. Tijdens dit promotietraject is de prospectieve CODE 7 studie opgestart en dataverzameling volbracht. Kandidaten voor epilepsiechirurgie met een verdenking van mMCD of FCD werden geïncludeerd en ondergingen een aanvullende preoperatieve 7T MRI-scan. Het weefsel dat tijdens een operatie werd verkregen, werd ex vivo gescand op de 7T MRI, met driedimensionale resoluties tot 0,2 $\mathrm{mm}$. De in vivo en ex vivo MRI-beelden zijn gecoregistreerd met gedigitaliseerde histologische coupes door middel van software ontwikkeld door leden van het onderzoeksteam. Dit betekent dat verschillende beelddata in één zelfde driedimensionale ruimte zijn geplaatst, ten behoeve van één-op-éen vergelijkingen. De ontwikkeling van de methodologische opzet is beschreven in hoofdstuk 7. De eerste resultaten van de CODE 7 studie zijn veelbelovend en in het nog lopende onderzoek streven we ernaar om verder te verhelderen hoe specifieke MRIkenmerken gerelateerd zijn aan verschillende histologische kenmerken. 


\section{Algemene Discussie}

Chirurgische behandeling van medicatie-resistente epilepsie bij patiënten met mMCD of FCD is de enige therapie met kans op aanvalsvrijheid en kent een hoge succeskans en lage risico's. Ondanks de relatief lagere succeskans bij de subtielere afwijkingen in mMCD en FCD type I dient chirurgie overwogen te worden. De kans op daadwerkelijk bereiken van aanvalsvrijheid na een operatie wordt bepaald door volledige verwijdering van de epileptogene zone, en voorspellende factoren die daarmee samenhangen. Wij onderzochten de relatie tussen patiënteigenschappen, histologische typering van mMCD/FCD en de kans op een succesvolle chirurgische behandeling. Volledige aanvalsvrijheid op lange termijn werd bereikt in 58\% met FCD en 32\% met mMCD. Onze bevindingen ondersteunen het onderscheid tussen mMCD, FCD I en FCD II subtypes vanwege verschillen in klinische uitingsvormen.

In de toekomst zijn er meer grote studies met een lange follow-up nodig om met volledige zekerheid predictiemodellen te maken voor aanvalsvrijheid in relatie tot histologische subtypes. Een vereiste is een goede reproduceerbaarheid van de histologische classificaties door verschillende beoordelaars. Er zijn nog verschillen in rapportage van de verschillende mMCD/FCD subtypes, mogelijk door verschil in gebruik van immunohistochemische technieken. Er is met name over mMCD als separate entiteit nog een debat gaande. Nieuwe inzichten en bijvoorbeeld inzet van genetische en moleculaire typeringen zouden de classificatie verder kunnen doen ontwikkelen.

Veel patiënten met epilepsie ten gevolge van mMCD of FCD hebben cognitieve beperkingen. In overeenkomst met eerdere studies vonden we een associatie tussen een vroeg begin van epilepsie en de ernstigere cognitieve beperkingen. Ook had een langere epilepsieduur een negatief effect op de cognitie. Het bleek dat patiënten met FCD type I ernstigere cognitieve problemen hadden, vermoedelijk door de meer diffuse aard van de afwijkingen. We vonden tegen onze verwachtingen in geen groter herstel in cognitie bij patiënten met aanvalsvrijheid en medicatievrijheid na chirurgie. Dat we dit in onze studie niet konden bevestigen is mogelijk een gevolg van de relatief korte follow-up duur van 2 jaar. Langer vervolgen van patiënten zal een beter beeld geven van de postoperatieve effecten op cognitie en samenhang met histologische subtypes.

De laatste decennia is er een verschuiving naar opereren op steeds jongere leeftijd bij patiënten met medicatie-resistente epilepsie, met name bij focale ontwikkelingsstoornissen. De hoop op preservatie van cognitieve functies is een sterk argument hiervoor. Er dient gestreefd te worden naar zo korte mogelijke tijd tussen het ontstaan van epilepsie en de chirurgische behandeling, waarbij uiteraard de kwaliteit en grondigheid van het diagnostische proces optimaal moet zijn. 
Verbetering van laesiedetectie en -begrenzing is één van de manieren om kwaliteit van de zorg voor deze patiëntgroep te verbeteren. Een technische ontwikkeling met potentie is 7T MRI, waarmee beeldvorming met hogere resolutie en contrast mogelijk is, ten opzichte van de huidige standaard - 3T MRI. Wij vonden dat bij multidisciplinaire beoordeling van 7T MRI, er bij $23 \%$ van de patiënten afwijkingen werden gevonden die niet waren gedetecteerd op de eerdere beeldvorming op lagere veldsterkte. Deze bevindingen hadden invloed op de klinische besluitvorming. Echter, bij de meerderheid van de patiënten leidde 7T MRI niet tot identificatie van een eerder verborgen afwijking. Dit is deels te verklaren doordat 7T MRI nog vroeg in de ontwikkeling is. Het optreden van diverse soorten artefacten beperken de beoordeelbaarheid van de scan. Daarnaast is de klinische ervaring van clinici nog relatief beperkt. De verwachting is dat de voortdurende technologische ontwikkeling de accuratesse van de beoordeling nog verder zal verbeteren. De inzet van 7T MRI lijkt zinvol te zijn bij een geselecteerde groep patiënten zonder aantoonbare structurele afwijkingen met een sterke klinische verdenking op locatie-gebonden epilepsie. Niet-structurele oorzaken moeten al zijn uitgesloten en, indien beschikbaar, dienen computergestuurde beeldanalyses te zijn uitgevoerd op de eerder gemaakte beeldvorming.

De $T^{2 *}$ sequentie op 7T MRI suggereerde dat afwijkende vaatstructuren als mogelijke nieuwe marker voor subtiele mMCD/FCD kunnen dienen. Onze vervolgstudie toonde echter geen toegenomen vaatdichtheid in MMCD of FCD ten opzichte van normaal hersenweefsel. Wel zagen we bij mMCD, FCD en tubers een hogere vaatdichtheid in de laesie ten opzichte van omliggende weefsel. Een verklaring voor het MRI-vaatpatroon kan mogelijk worden gezocht in hemodynamische variatie en daarbij horende veranderingen in bloedzuurstofspiegel. Verder onderzoek is nodig om te bepalen of BOLD fMRI (dynamisch MRI-onderzoek gebaseerd op visualisatie van variaties in bloedzuurstofspiegel) misschien van waarde kan zijn in de detectie van anderszins MRI-negatieve dysplasie.

De vraag wat er nodig is voor $100 \%$ detectie, als dat al ooit mogelijk gaat zijn, is nog niet opgelost. Moeten we ons concentreren op spatiale resolutie of op het verbeteren van het weefselcontrast? Of moeten scantechnieken worden aangepast om weefselcontrast te optimaliseren op basis van specifieke histologische veranderingen in de verwachte afwijking? Deze vragen waren de aanleiding voor het opstarten van de CODE 7 studie. Dit proefschrift beschrijft de eerste bevindingen wat betreft histologische verschillen en de kans op een succesvolle chirurgische behandeling, gebruik makend van data verzameld in de CODE 7 studie. Onze toekomstige analyses dragen hopelijk bij aan verdere verbetering van de identificatie en begrenzing van subtiele afwijkingen.

Optimale epilepsiezorg zal niet alleen moeten komen van technische innovatie maar ook de integratie van data van de verschillende aanvullende onderzoeken en van een multidisciplinaire benadering waarbij er een nauwe samenwerking is tussen neurologen, 
elektrofysiologie, neurochirurgen, technisch geneeskundigen, neuropsychologen, en radiologen. De grote ziektelast van patiënten met medicatie-resistent epilepsie maakt epilepsiechirurgie een van de meest dankbare therapieën in de moderne neurologie. Dankzij de intense inspanning van vele wetenschappers en zorgprofessionals wereldwijd valt er nog veel verbetering van de zorg te verwachten. 


\section{Acknowledgements}

\section{All chapters}

This work was supported by the Dutch Epilepsy Foundation (grant 12-12).

\section{Chapter 3}

This work was supported by KIKA (Stichting Kinderen Kankervrij, The Netherlands; AM, EA); Stichting AMC Foundation, The Netherlands (EA); Stichting TSC Fonds, The Netherlands (EA); the Austrian Science Fund (FWF, no. J3499; AM); the European Union 7th Framework Programme: acronym EPISTOP (grant agreement no. 602391; AM, EA).

The authors would like to thank our colleagues from the department of neuropsychology and Banu Swampillai for their contributions to the collection of neuropsychologic testing scores and clinical data.

\section{Chapter 4}

The authors thank Wim Spliet, Wim van Hecke, and Angelika Mühlebner for performing histopathology and providing feedback considering correspondence with imaging findings.

\section{Chapter 5}

The authors thank Fredy Visser for technical support. 


\section{Dankwoord}

Het ei is gelegd. De laatste 7 jaar was de PhD een rode draad in mijn leven, met ook een aantal langere periodes van klinisch werk. Zoals dat hoort bij een dergelijk onderneming waren er zowel hoogte als dieptepunten en het is zondermeer een leerzame en vormende tijd geweest. Naarmate het onderzoek vorderde werd het steeds duidelijker dat samenwerking en de hulp van collega's en niet-collega's essentieel is.

Er zijn een hoop mensen die ik zeer hartelijk wil bedanken voor hun hulp. Het is bijna onvermijdelijk dat ik toch verzuim iemand te bedanken voor een waardevolle bijdrage; weet dat het echt wel gewaardeerd werd.

Allereerst wil ik alle patiënten bedanken voor jullie deelname aan mijn onderzoek, vaak met grote interesse en enthousiasme, ondanks dat de reden dat jullie überhaupt in aanmerking kwamen grote weerslag heeft op jullie leven. Zonder jullie deelname was dit proefschrift niet mogelijk geweest.

Many thanks to members of the assessment committee prof. dr. Elly Hol, prof. dr. Ingmar Blümcke, prof dr. ir. Max Viergerver, prof. dr. Yasin Temel and dr. Albert Colon for taking time and effort for the appraisal of my thesis, I feel honoured.

Ik wil mijn grote dank uitspreken naar mijn begeleidingsteam: mijn promotoren prof $\mathrm{dr}$. Kees Braun, prof. dr. Eleonora Aronica en copromotoren dr. Pieter van Eijsden en dr. Cyrille Ferrier. Bedankt voor het vertrouwen om mij te laten starten als promovendus onder jullie hoede en alle hulp over de laatste jaren. Ik kon altijd vertrouwen op snelle en nuttige feedback, en ook af en toe de schop onder de kont was nuttig op momenten dat ik weer verzandde in de details.

Beste Kees, ik heb bewondering voor je bevlogenheid in zowel de klinische en wetenschappelijke kant van de epilepsiezorg. Ik vind het steeds bijzonder hoe je met zo een overvolle agenda altijd tijd hebt gemaakt om mij snel weer op weg te helpen als dat nodig was. Beste Eleonora, ook op jouw lab heb ik me meer dan welkom gevoeld. Je passie voor het vak is inspirerend. Ik heb veel van je mogen leren over de neuropathologie. De bliksemsnelheid waarmee je altijd mijn onderzoeksvragen hebt beantwoord is bewonderenswaardig, of het nou ging over een korte vraag of een volledig figuur.

Beste Cyrille, jij hebt me in 2012 uitgenodigd om dit onderzoek te starten, waarvoor dank. Ik wil je ook bedanken voor hoe je me hebt betrokken in de werkgroep epilepsiechirurgie en het vertrouwen dat je me gaf wat betreft mijn rol met betrekking van de 7T MRI daarin. Mijn meest waardevolle werkervaringen als arts blijven ook de cases waarin jij me meenam 
en onze gedeelde liefde voor muziek en de (epilepsie)chirurgie samenkwamen, of juist botsten voor de patiënten.

Beste Pieter, jij weet steeds precies de essentie uit te distelleren en een boodschap krachtiger te verpakken, vaak precies wat ik nodig had. Ook mijn dank voor de gezelligheid en samenwerking tijdens mijn tijd als assistent bij de neurochirurgie en de daarbij horende ski-trips.

Beste Angelika Mühlebner, ik betwijfel of alle pathologiehoofdstukken in dit proefschrift er waren geweest zonder jouw inzet, veel dank hiervoor!

Wim van Hecke, bedankt voor de tomeloze energie en hulp bij alle zaken rondom verwerken van weefsel bij de CODE 7 studie. Dit samen uitvogelen en de ontmoetingen hieromheen waren een groot plezier. Wim Spliet, hartelijk dank voor de altijd fijne samenwerking op de pathologie. Sander Diederen, ik ben enorm blij dat jij op een gegeven moment aan boord bent gestapt bij het CODE7 project. Los van de prettige collega in de kliniek en wetenschap, was jouw input precies wat er nodig was om het CODE 7 project tot iets moois te brengen. Herke Jan Noordmans, ook bedankt voor jouw rol in dit project, en de zeer nuttige automatisering met behulp van jouw software tools.

Banu Swampillai, het was leuk en leerzaam om je te begeleiden tijdens je wetenschapsstage. Heel veel dank voor je waardevolle bijdragen aan hoofdstuk 2 . Andrew de Neef, bedankt voor je werk als student voor hoofdstuk 6 , het was een belangrijke kick-start om uiteindelijk tot een publicatie te komen.

Peter Gosselaar en Peter van Rijen, grote dank voor jullie medewerking en de CODE 7 studie en de prachtige en bloc resecties waar ik altijd op kon rekenen. Ook tijdens mijn tijd bij de neurochirurgie heb ik veel van jullie mogen leren.

Jaco Zwanenburg, je hulp bij het ex vivo scannen voor de CODE 7 studie was absoluut onmisbaar, dank!

Fredy Visser en Hans Hoogduin, jullie heb ik vaak nodig gehad rondom de patiëntscans op de 7T MRI, ik ben blij dat ik onderweg het een en ander van jullie heb mogen leren over MRI-technieken.

Prof. Jeroen Hendrikse en prof. Peter Luijten, dank voor de ruimte die ik heb gekregen op de 7T MRI op mijn onderzoek uit te voeren, en de wetenschappelijke feedback.

Gérard de Kort, bedankt voor je bijdrage aan hoofdstuk 4 en de samenwerking in de LWEC. Monique van Schooneveld, bedankt voor de samenwerking in het cognitiestuk.

Ingmar, Aidin en Dimitri, het was erg fijn dat jullie vaak op korte afstand zaten te werken in het coilslab en altijd bereid waren om te helpen op de 7T MRI als er weer een voor mij onbegrijpelijk technisch probleem voordeed.

MRI-laboranten Johan, Gerrit, Niels, Daniël, Gideon, Myrthe, Erik, Guus, Cindy en Greet, ik heb het erg gezellig en leerzaam gevonden om met jullie een kleine honderd 7T MRI-scans bij epilepsiepatiënten te maken. 
Graag wil ik leden van de epilepsiechirurgiewerkgroep Janine Ophorst, Truus de Groot, Frans Leijten, Tineke Gebbink, Floor Jansen, Karin Geleijns, Peter van Rijen, Peter Gosselaar, Mireille Bourez, Monique Hobbelink, Louis Wagner, Guido Widman, Steven Claus, Dimitri Velis, Annette Hospes, Ilse van Straaten, Hanneke Ronner, Gérard de Kort, Maeike Zijlmans, Jack Zwemmer, Hilde Braakman, Albert Colon, Monique van Schooneveld, Martine Van Zandvoort, Renate van Regteren en Jaap Reijneveld graag bedanken voor de fijne samenwerking, en de medewerking aan mijn onderzoekprojecten.

Hélène Willems, bedankt voor het me door het papieren doolhof sluizen en je oprechte interesse.

Mede-epilepsieonderzoekers, bedankt voor de wetenschappelijke feedback en journal clubs en nog meer voor de gezelligheid tijdens congressen, gezamenlijk lunches en uitjes: Herm, Dorien, Nicole, Lotte, Bart, Eric, Kim, Jolien, Maryse, Willemieke, Brian, Marij, en in het bijzonder mijn kamergenoten, Kora, Anne, Matea en Hanna. Anne ook bedankt voor je feedback in de afrondende fase van het proefschrift. Suzanne en Willem bedankt voor advies over ex-vivo scannen en beeldanalyses.

De neuropathologieclub in het AMC, Anand, Jackelien, Erwin, Jasper, Diede, Anatoly, Anika, James, Avanita, het was wat onvoorspelbaar wanneer ik er zou zijn maar ik werd altijd zeer warm verwelkomd, dank! Renee en Jasper ontzettend bedankt voor al de hulp omtrent de histologische coupes en mij wegwijs maken in het lab.

Onno de Boer en Eelco Roos bedankt voor het digitaliseren van de histologische coupes.

Ook op het pathologielab in het UMCU was ik altijd welkom en kon ik rekenen op vriendelijke hulp, zelfs nadat ik de uitsnijkamer onklaar had gemaakt door wat ongelukkige knoeierij met de Agarose-gel, met een verstopte afvoer als gevolg, oeps. Dank aan Jan-Willem, Jan, Ronny, Jefrey, Nienke, Harry, Will, Frederic, Jessica, Hetty, Eva, Sarina, Sibel, Tinus, Lynn, Joost, Marieke en Juul.

Imaging onderzoekers van het Q2 gebouw: Alex, Cyril, Anita, Arjen, Katharina, Michaela, Deji, Janot, Arjan, Bart, Catalina, Erwin, Ingmar, Jannie, Jeroen, Lennart, Lisa, Oscar, Quincy, Tijl, Anja, Nikki, Mike, Stefano, Martijn, dank voor de 7T MRI-adviezen, gezellige uitjes, maandagse fietsavonden, en de boulder-avonden. Ook het ISMRM-congres in Singapore met veel van jullie was een hoogtepuntje.

Wellicht is het achteraf gezien een toeristische carrière-route geweest, maar ik heb de afgelopen jaren veel geleerd en gelachen als arts-assistent bij de neurochirurgie en neurologie in het UMC Utrecht, neurochirurgie in het Amsterdam UMC locatie VU en in het Alexander Monro Borstkanker Ziekenhuis, alle oud-collega's hartelijk bedankt. 
Collega's in het Meander MC, bedankt voor de leerzame en gezellige afgelopen twee jaar bij de radiologie en nucleaire geneeskunde. Mede dankzij jullie is de opleiding zo ontzettend leuk. Jasper, bedankt dat je me als opleider ruimte hebt gegeven om de COVID19-tijd te benutten voor afronding van mijn proefschrift.

Ook het team van Spotify verdient krediet, eigenlijk was de muziek nooit uit tijdens het onderzoek: we hebben zo een 50.000-70.000 minuten per jaar samen beleefd volgens de jaarlijkse overzichten. Het muziekvlammetje bleef zo stevig aangewakkerd. Kris, ook jouw zanglessen waren een essentiële muzikale afleiding om het wetenschappelijke werk op m'n best te kunnen doen. Direct gevolg zijn nu de immer epische sessies met de Fuzzboys.

Ik mag mezelf gelukkig prijzen met een hoop waardevolle vriendschappen, ontstaan in de wieg, op een festivalwijde of op het hockeyveld als F-jes; op het Anne van Rijn college of de geneeskundefaculteit; in de moshpit of lindy hop balzaal; binnen Jaarclub Uitzonderring; tijdens coschappen in Kaapstad en Brussel; en de laatste jaren in eigen stadsie Utrecht. Hoewel de meesten niet direct betrokken zijn geweest, waren jullie toch een zeer belangrijke factor in het uiteindelijk succesvol voltooien van deze promotie. Alle feestjes, concerten, theatervoorstellingen, festivals, reizen, weekendjes weg, ski-trips, kitesurf-sessies, LAN-parties, bootcampsessies, dansavonden, band-sessies, diners en bar-hangerij waren onmisbare bronnen van nieuwe energie.

Paranimfen Betto en Laura, ik ben blij dat jullie me willen vergezellen tijdens de verdediging als academische tijgers en middelbare school buddies. Dank voor de hulp in de afrondende fase.

Aan beiden kanten ben ik dankbaar voor een grote, erg warme en trouwe familie Veersema en Serkei. Daarnaast heb ik dankzij Caroline er nog geweldige schoonfamilies bij in Brabant en Vlaanderen.

Lieve Nils, broeder, maatje, collega, ik ben blij om alle geplande en spontane sportieve, muzikale of gewoon gezellige uitjes in binnen- en buitenland. Bedankt voor je nuttige feedback. Robin, jij bent een geweldige uitbreiding van het gezin.

Mijn ouders, wat heb ik ook in mijn PhD ontzettend veel aan jullie gehad. Ik heb veel aan jullie te danken, jullie gaven ons nog heel veel meer dan alleen een warm nest en onvoorwaardelijke steun en liefde. Mam, je bent werkelijk de liefste en meest zorgzame persoon die ik ken. Pap, je bent als persoon én arts een groot voorbeeld.

Liefste Caroline, ik ben zo gelukkig om jou al sinds 2009 in mijn leven te hebben en ik ben stapelgek op jou. Je hebt alle fases van dit proefschrift van heel dichtbij meegemaakt en 
mij vaker dan ik zou willen toegeven over een hindernis geholpen, ik kan je niet genoeg bedanken. Ik ben blij en trots dat de omslag een kunstwerk van jouw hand is. Sinds dit jaar zijn we met z'n drieën, mijn geluk kan niet op! Lieve Coco, jij begint én eindigt elke dag met een grootse lach, en het is onweerstaanbaar aanstekelijk. 


\section{Curriculum Vitae}

Tim Veersema was born on 1 July 1987 in Amsterdam, the Netherlands. After completing a bilingual trajectory (tweetalig VWO) at the Anne van Rijn College secondary school in Nieuwegein and graduating with honours, he started his Medicine study at the University of Maastricht in 2005. During his studies Tim had the opportunity to do medical internships in Kubang Kerian in northern Malaysia, Brussels and Capetown. It was during this neurology rotation in South Africa were Tim developed interest in the nervous system and brain in particular. After graduating in 2011, Tim travelled five months through South America with his girlfriend Caroline, after which he started working as resident at the department of Neurosurgery in the University Medical Center Utrecht. Consecutively he started his PhD under

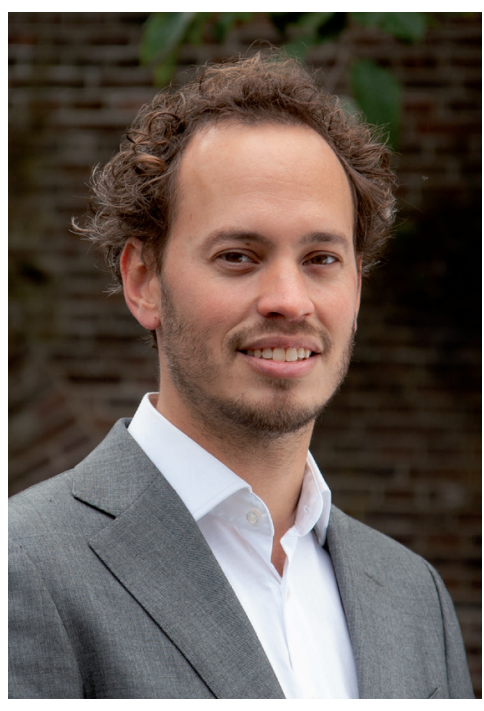
supervision of Kees Braun (neurology), Eleonora Aronica (neuropathology, Amsterdam UMC, location AMC), Cyrille Ferrier (clinical neurophysiology) and Pieter van Eijsden (neurosurgery). During his years as researcher Tim did weekend, evening and night shifts for neurosurgery at the UMCU, was a night-doctor at the Alexander Monro Breast Cancer Hospital, Bilthoven and worked as a resident at the department of neurosurgery of the Amsterdam AMC, location VU Medical Center. January 2019 he started his specialist training in radiology and nuclear medicine in the Meander Medical Center in Amersfoort and University Medical Center Utrecht. Caroline and Tim live in Utrecht, together with their daughter Coco, born January 2020. 


\section{List of publications}

\section{In this thesis}

Tim. J. Veersema, Pieter Van Eijsden, Peter H. Gosselaar, Jeroen Hendrikse, Jaco M. Zwanenburg, Wim G.M. Spliet, Eleonora Aronica, Kees P.J. Braun, Cyrille H. Ferrier 7 tesla T2*-Weighted MRI as a Tool to Improve Detection of Focal Cortical Dysplasia - A Case Series.

Epileptic Disorders. 2016 Sep;18(3):315-323.

Tim J. Veersema, Cyrille H. Ferrier, Pieter van Eijsden, Peter H. Gosselaar, Eleonora Aronica, Fredy Visser, Jaco M. Zwanenburg, Gerard A.P. de Kort, Jeroen Hendrikse, Peter R. Luijten, Kees P.J. Braun.

Seven tesla MRI improves detection of focal cortical dysplasia in patients with refractory focal epilepsy.

Epilepsia Open. 2017;4-10.

Tim. J. Veersema, Banu Swampillai, Cyrille H. Ferrier, Pieter van Eijsden, Peter H. Gosselaar, Peter C. van Rijen, Wim G.M. Spliet, Angelika Mühlebner, Eleonora Aronica, Kees P.J. Braun. Long-term Seizure Outcome after Epilepsy Surgery in Patients with Mild Malformation of Cortical Development and Focal Cortical Dysplasia.

Epilepsia Open. $2019 ; 4(1): 170-5$

Tim J. Veersema, Monique M. J. van Schooneveld, Cyrille H. Ferrier, Pieter van Eijsden, Peter H. Gosselaar, Peter C. van Rijen, Wim G.M. Spliet, Angelika Mühlebner, Eleonora Aronica, Kees P.J. Braun.

Cognitive functioning after epilepsy surgery in children with mild Malformation of Cortical Development and Focal Cortical Dysplasia.

Epilepsy \& Behavior. 2019;94:209-15.

Tim. J. Veersema, Andrew de Neef, Jackelien van Scheppingen, Cyrille H. Ferrier, Pieter van Eijsden, Peter H. Gosselaar, Peter C. van Rijen, Wim G.M. Spliet, Kees P.J. Braun, Angelika Mühlebner, Eleonora Aronica.

Changes in vascular density in resected tissue of 97 patients with mild malformation of cortical development, focal cortical dysplasia or TSC- related cortical tubers.

Int J Dev Neurosci. 2019;79:96-104. 
Tim J. Veersema*, Sander J.H. Diederen*, Cyrille H. Ferrier, Pieter van Eijsden, Jaco J.M. Zwanenburg, Herke Jan Noordmans, Peter .H. Gosselaar, Peter C. van Rijen, Wim van Hecke, Angelika Mühlebner, Kees P. Braun\#, Eleonora M.A. Aronica\#,

Correlation between in vivo and ex vivo multimodal high field MRI and histopathological parameters in cortical dysplasias.

in preparation

* contributed equally as first authors

\# shared senior authorship

\section{Other}

Avanita S. Prabowo, Anand M. Iyer, Tim J. Veersema, Jasper J. Anink, Antoinette Y. N. Schouten-van Meeteren, Spliet WG, Peter C. van Rijen, Cyrille H. Ferrier, David Capper, Maria Thom, Eleonora Aronica.

BRAF V600E mutation is associated with mTOR signaling activation in glioneuronal tumors. Brain Pathol. 2014 Jan;24(1):52-66.

Avanita S. Prabowo, Anand M. Iyer, Tim J. Veersema, Jasper J. Anink, Antoinette Y. N. Schouten-van Meeteren, Wim G.M. Spliet, Peter C. van Rijen, Cyrille H. Ferrier, Maria Thom, Eleonora Aronica.

Expression of neurodegenerative disease-related proteins and caspase- 3 in glioneuronal tumours.

Neuropathol Appl Neurobiol. 2015 Feb;41(2):e1-e15.

Avanita S. Prabowo, Hinke F. van Thuijl, Ilari Scheinin, Daoud Sie, Hendrik F. van Essen, Anand M. Iyer, Wim G.M. Spliet, Cyrille H. Ferrier, Peter C. van Rijen, Tim J. Veersema, Maria Thom, Antoinette Y. N. Schouten-van Meeteren, Jaap C. Reijneveld, Bauke Ylstra, Pieter Wesseling, Eleonora Aronica.

Landscape of chromosomal copy number aberrations in gangliogliomas and dysembryoplastic neuroepithelial tumours.

Neuropathol Appl Neurobiol. 2015 Oct;41(6):743-55

Angelika Mühlebner, Anand M. Iyer, Jackelien van Scheppingen, Jasper J. Anink, Floor E. Jansen, Tim J. Veersema, Kees P.J. Braun, Wim G.M. Spliet, Wim van Hecke, Figen Söylemezoğlu, Martha Feucht, Pavel Krsek, Josef Zamecnik, Christian G. Bien, Tilman Polster, Roland Coras, Ingmar Blümcke, Eleonora Aronica.

Specific pattern of maturation and differentiation in the formation of cortical tubers in tuberous sclerosis Complex (TSC): evidence from layer-specific marker expression.

J Neurodev Disord. 2016 Apr 1;8:9 
Rick H.G.J. van Lanen, Linda A.A. Jacobi-Postma, Tim J. Veersema, Onno P.M. Teernstra, Jim T.A. Dings.

Clinical and Radiological Outcomes of Intracranial Aneurysm Clipping Aided by Transit Time Flowmetry. World Neurosurg. 2020;136:e660-70.

\section{Presentations}

Tim. J. Veersema, Cyrille H. Ferrier, Pieter Van Eijsden, Peter H. Gosselaar, Jeroen Hendrikse, Wim G.M. Spliet, Eleonora Aronica, Kees P.J. Braun

Focal cortical dysplasia on 7 tesla susceptibility weighted magnetic resonance imaging.

Oral presenation. 11th European Congress on Epileptology, Stockholm, Sweden, 29 June - 3 July, 2014.

Tim. J. Veersema, Pieter Van Eijsden, Peter H. Gosselaar, Jeroen Hendrikse, Jaco M. Zwanenburg, Wim G.M. Spliet, Eleonora Aronica, Kees P.J. Braun, Cyrille H. Ferrier.

7 tesla T2*-Weighted MRI as a Tool to Improve Detection of Focal Cortical Dysplasia: a Case Series.

Oral presentation and poster. Dutch League Against Epilepsy, Chapter Scientific Research winter meeting 2015, Amsterdam, the Netherlands, 11 March 2015.

Best Clinical Research Award.

Tim J. Veersema, Cyrille H. Ferrier, Pieter van Eijsden, Peter H. Gosselaar, Fredy Visser, Jaco M. Zwanenburg, Hans Hoogduin, Gerard A.P. de Kort, Jeroen Hendrikse, Peter R. Luijten, Kees P.J. Braun.

7 tesla MRI in the pre-surgical evaluation of 26 patients with focal epilepsy.

Oral presentation and poster. Dutch League Against Epilepsy, Chapter Scientific Research winter meeting 2016, Amsterdam. 28 January 2016.

Best Clinical Research Award.

Tim J. Veersema, Cyrille H. Ferrier, Pieter van Eijsden, Peter H. Gosselaar, Fredy Visser, Jaco M. Zwanenburg, Hans Hoogduin, Gerard A.P. de Kort, Jeroen Hendrikse, Peter R. Luijten, Kees P.J. Braun.

7 tesla MRI in the pre-surgical evaluation of 19 children with focal epilepsy.

Poster. International Child Neurology Congress 1 - 5 mei 2016, Amsterdam.

Tim J. Veersema, Cyrille H. Ferrier, Pieter van Eijsden, Peter H. Gosselaar, Fredy Visser, Jaco M. Zwanenburg, Hans Hoogduin, Gerard A.P. de Kort, Jeroen Hendrikse, Peter R. Luijten, Kees P.J. Braun.

7 tesla MRI in the pre-surgical evaluation of 36 patients with focal epilepsy. 
Oral presentation. International Society for Magnetic Resonance in Medicine meeting, 7 - 13 May 2016, Singapore.

Tim J. Veersema, Cyrille H. Ferrier, Pieter van Eijsden, Peter H. Gosselaar, Fredy Visser, Jaco M. Zwanenburg, Hans Hoogduin, Gerard A.P. de Kort, Jeroen Hendrikse, Peter R. Luijten, Kees P.J. Braun.

7 tesla MRI in the pre-surgical evaluation of patients with refractory focal epilepsy oral presentation. 12th European Congress on Epileptology, 11 - 15 September 2016, Prague. Selected for 'highlight session'. 

